\title{
Acknowledging Patient Heterogeneity in Health Technology Assessment : Towards Personalized Decisions in Innovative Radiotherapy Treatments
}

Citation for published version (APA):

Ramaekers, B. L. T. (2013). Acknowledging Patient Heterogeneity in Health Technology Assessment : Towards Personalized Decisions in Innovative Radiotherapy Treatments. [Doctoral Thesis, Maastricht University]. Datawyse / Universitaire Pers Maastricht. https://doi.org/10.26481/dis.20130913br

Document status and date:

Published: 01/01/2013

DOI:

10.26481/dis.20130913br

Document Version:

Publisher's PDF, also known as Version of record

Please check the document version of this publication:

- A submitted manuscript is the version of the article upon submission and before peer-review. There can be important differences between the submitted version and the official published version of record.

People interested in the research are advised to contact the author for the final version of the publication, or visit the DOI to the publisher's website.

- The final author version and the galley proof are versions of the publication after peer review.

- The final published version features the final layout of the paper including the volume, issue and page numbers.

Link to publication

\footnotetext{
General rights rights.

- You may freely distribute the URL identifying the publication in the public portal. please follow below link for the End User Agreement:

www.umlib.nl/taverne-license

Take down policy

If you believe that this document breaches copyright please contact us at:

repository@maastrichtuniversity.nl

providing details and we will investigate your claim.
}

Copyright and moral rights for the publications made accessible in the public portal are retained by the authors and/or other copyright owners and it is a condition of accessing publications that users recognise and abide by the legal requirements associated with these

- Users may download and print one copy of any publication from the public portal for the purpose of private study or research.

- You may not further distribute the material or use it for any profit-making activity or commercial gain

If the publication is distributed under the terms of Article $25 \mathrm{fa}$ of the Dutch Copyright Act, indicated by the "Taverne" license above, 


\title{
Acknowledging Patient Heterogeneity in Health Technology Assessment
}

\author{
Towards Personalized Decisions \\ in Innovative Radiotherapy Treatments
}

Bram Ramaekers 


\section{Production}

ISBN: $\quad 9789461592422$

Cover: Jennifer Strijbos (met dank aan Michiel Schmeitz)

Printed by: Datawyse | Universitaire Pers Maastricht

\section{Copyrights}

(C) Copyright Bram Ramaekers, Maastricht 2013

Chapter 2: $\quad$ Value Health, John Wiley \& Sons Inc., 2013

Chapter 3: $\quad$ Canc Treat Rev, Elsevier Inc., 2011

Chapter 4: $\quad$ Oral Oncol, Elsevier Inc., 2011

Chapter 5: $\quad$ Int J Radiat Oncol Biol Phys, Elsevier Inc., 2013

Chapter 6: J Thorac Oncol, Wolters Kluwer Health Inc. 2013

All rights reserved. No part of this thesis may be reproduced or transmitted in any form or by any means, electronic or mechanical, including photocopying, recording or any information storage or retrieval system, without prior written permission of the holder of the copyright. 


\title{
Acknowledging Patient Heterogeneity in Health Technology Assessment
}

\author{
Towards Personalized Decisions \\ in Innovative Radiotherapy Treatments
}

\begin{abstract}
PROEFSCHRIFT
ter verkrijging van de graad van doctor aan de Universiteit Maastricht, op gezag van de Rector Magnificus Prof. dr. L.L.G Soete, volgens het besluit van het College van Decanen, in het openbaar te verdedigen op vrijdag 13 september 2013 om 10:00 uur
\end{abstract}

door

Bram Lambertus Theodorus Ramaekers

Geboren op 28 januari 1986 te Venlo

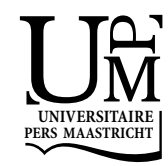




\section{Promotor}

Prof. dr. P Lambin

\section{Copromotores}

Dr. J.P.C. Grutters (Universitair Medisch Centrum St Radboud, Nijmegen)

Dr. M.A. Joore

Dr. M. Pijls-Johannesma

\section{Beoordelingscommissie}

Prof. dr. B. Kremer (voorzitter)

Dr. A.D.I. Van Asselt

Dr. F. Hoebers

Prof. dr. Y. Lievens (Universitair Ziekenhuis Gent)

Financial support by Carl Zeiss and Roche Nederland B.V. for the publication of this thesis is gratefully acknowledged. 


\section{Contents}

PART I The role of patient heterogeneity in economic evaluation

Chapter 1 General introduction and outline

Chapter 2 How should we deal with patient heterogeneity in economic evaluation: a systematic review of pharmacoeconomic guidelines

PART II Acknowledging heterogeneity in health technology assessment in radiotherapy

Chapter 3 Systematic review and meta-analysis of radiotherapy in various 45 head and neck cancers: Comparing photons, carbon-ions and protons

Chapter 4 The impact of late treatment-toxicity on generic health-related 83 quality of life in head and neck cancer patients after radiotherapy

Chapter 5 Protons in head and neck cancer: bridging the gap of evidence 101

Chapter 6 Cost-effectiveness of modified fractionation radiotherapy 123 versus conventional radiotherapy for unresected non-small cell lung cancer patients.

\section{PART III Discussion}

Chapter 7 General discussion

Samenvatting

Publications

Dankwoord 



\section{PART I}

The role of patient heterogeneity in economic evaluation

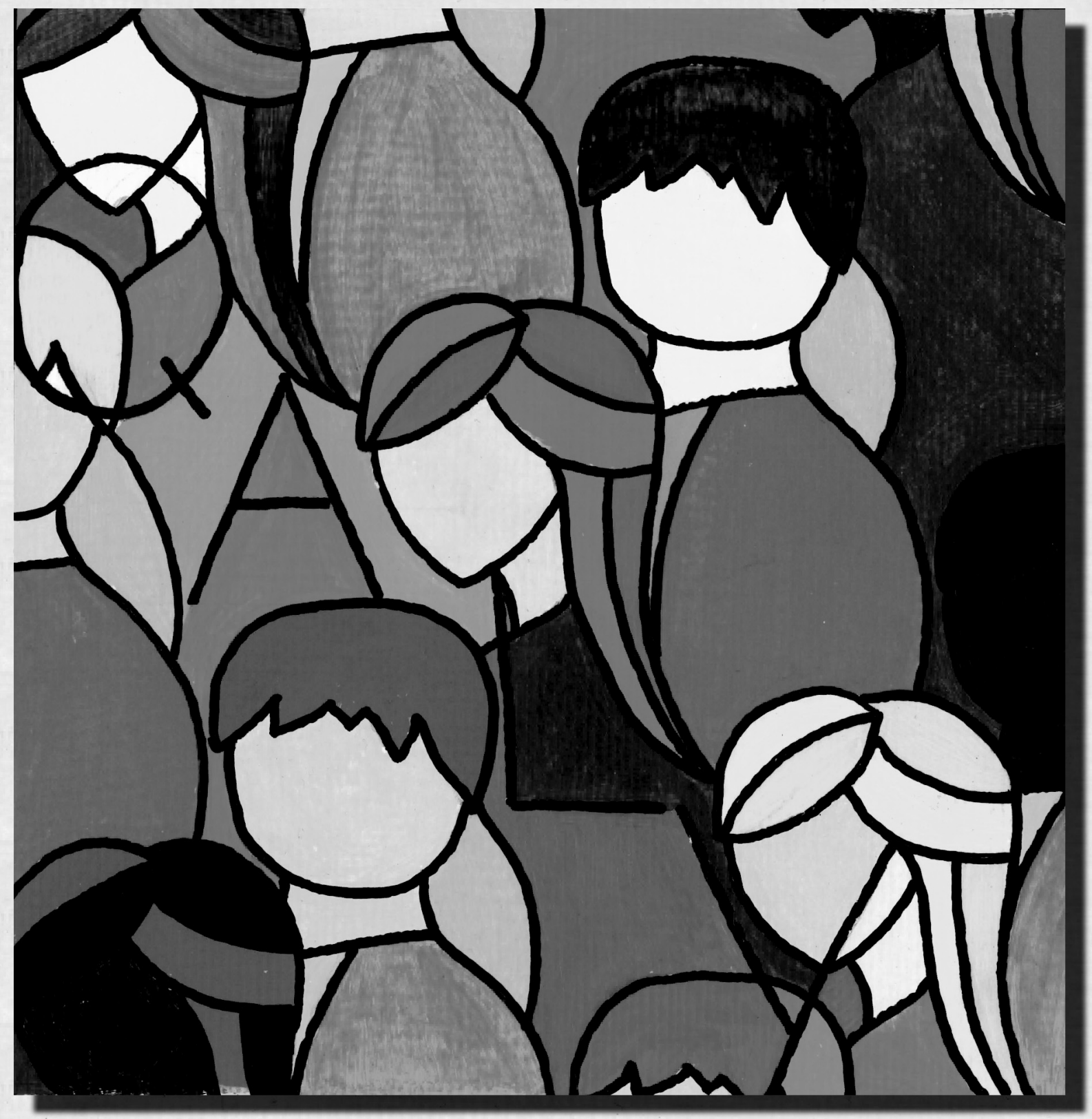





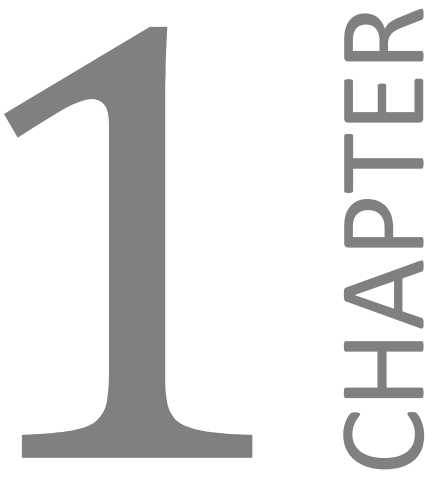

GENERAL INTRODUCTION AND OUTLINE 
CHAPTER 1 
Despite the high degree of similarities that bind humanity together as a species, considerable diversity exists between individual patients. ${ }^{1}$ All patients are individuals and often respond differently to identical treatments despite similar diagnosis. ${ }^{2}$ For instance in oncology, patients with similar histological features, stages and grades of cancer have a broad range of outcomes. Some patients may respond to treatment; others do not. ${ }^{3}$ This natural variation can be defined as variability. Unfortunately, the large part of this variability in treatment response is often not readily predictable before treatment. ${ }^{2}$ The part of this variability that can be explained by certain patient characteristics is defined as patient heterogeneity. These patient characteristics may include demographics (e.g. age, gender, income), preferences (e.g. attitude, beliefs, risk tolerance) and clinical characteristics (e.g. disease severity, disease history, genetic profile). ${ }^{4}$ Patient heterogeneity should be distinguished from treatment heterogeneity, which refers to differences in the nature of the treatment (e.g. differences in treatment dose or technique). Patient heterogeneity is increasingly acknowledged in clinical practice through anticipating on predictable differences in treatment response and aiming to tailor treatments to individual patients based on this information. ${ }^{3,5}$ It is considered one of the great challenges for $21^{\text {st }}$ century medicine to deliver effective therapies that are tailored to an individual ${ }^{6}$ to enable so-called personalized medicine; 'the right treatment for the right person'. The promise of personalized medicine is that it will improve treatment efficacy, reduce toxicity and minimize costs. ${ }^{7}$ This minimization of costs can be considered valuable in the light of the accelerating healthcare costs and the growing attention on costs of expensive novel medicines and technologies. Economic evaluations are increasingly employed to examine the costs of novel medicines and technologies in relation to its effects. Ideally this evaluation is based on all relevant evidence. Patient heterogeneity may affect different input parameters that are used in an economic evaluation: baseline risks, relative treatment effects, health state utility and resource utilization. ${ }^{4}$ Differences in unit costs, are more likely to result from differences between geographical regions and are thus unlikely to originate from differences between patients. ${ }^{4}$ Similarly as mentioned above for clinical practice, acknowledging patient heterogeneity in economic evaluations would be beneficial as it potentially improves the effectiveness and/or efficiency of health care. ${ }^{4}$ Patient heterogeneity is, however, often neglected in economic evaluations. ${ }^{8}$ This thesis focuses on the role of patient heterogeneity in economic evaluation. We hypothesize that it is feasible and informative to acknowledge patient heterogeneity in economic evaluation. 


\section{Health technology assessment}

Western societies are facing dramatically increasing healthcare costs. This includes the skyrocketing costs of cancer care, which are mainly caused by the ageing population and costly new treatments. ${ }^{9,10}$ Considering these escalating costs, the subsequent rising insurance premiums and the finite healthcare budgets, available resources should be allocated as efficient as possible and choices between healthcare technologies have to be made. This implies restricting reimbursement in the statutory package of insured care, to healthcare technologies that provide acceptable value for their money. Health Technology Assessments (HTA) is a field of research that aims to inform health policy makers in these decisions by examining the medical, economic, social and ethical implications of a medical technology in healthcare. ${ }^{11}$ In this context, a technology can be anything from preventive screening, pharmaceuticals, medical devices and surgical procedures to the organization of healthcare. ${ }^{11}$ Economic evaluation is a frequently used tool for HTA to compare the costs and consequences of different healthcare technologies. These consequences or effects are often expressed in terms of Quality Adjusted Life Years (QALYs). ${ }^{12}$ The QALY is a product of the quantity and the quality of life lived. It is a combined measure that captures both life expectancy and generic health-related quality of life (HRQOL) in a single value. HRQOL is often measured using disease-specific measures such as the European Organization for Research and Treatment of Cancer, Quality of Life Questionnaire-C 30 (EORTC-QLQC30). Although disease-specific measures of $\mathrm{HRQOL}$ are relevant for patients and physicians, their main disadvantage is the inability to compare HRQOL between different disease areas. In contrast, generic HRQOL can be compared in different disease areas. Generic HRQOL in terms of utility scores can be used to calculate QALYs. In this calculation life expectancy is corrected with quality weights, using utility scores, which are based on preferences for different outcomes and measured on an interval scale. ${ }^{12}$ Utility scores indicate the preference for a certain health state; the more preferable a health state, the more utility associated with it. ${ }^{12}$ These preferences can be measured from the patients' perspective by inquiring how patients value their own health status or from the general public perspective based on the preferences of society. Direct or indirect methods can be used to elicit utility scores for certain health states. Direct methods are often complex and time consuming. Indirect methods to elicit utility scores might be preferred to bypass these disadvantages and to measure utility scores from a societal perspective. ${ }^{12,13}$ Indirect methods include multi-attribute health status classification systems using generic preference questionnaires. The EuroQol-5D questionnaire ${ }^{14}$ (EQ-5D) is the most frequently used multi-attribute health status classification system. The EQ-5D consists of questions regarding five dimensions of generic HRQOL (mobility, self-care, 
usual activities, pain/discomfort and anxiety/depression). ${ }^{14}$ The answers to these questions can be combined to calculate utility scores using a scoring function based on the societal perspective. Calculated utility scores range from less than 0 (health state worse than death, severe problems in all five dimensions), through 0 (death) to 1 (full health, no problems in all dimensions). ${ }^{14}$

Ideally, economic evaluations consider all relevant evidence with regard to the costs and consequences and compare the full range of available treatment options. Meta-analyses are often necessary to satisfy these requirements and combine results from different studies or sources. For instance, meta-analysis can be used to synthesize available effectiveness data retrieved from multiple randomized controlled trials. Decision-analytical models can then act as an analytical framework to combine this evidence synthesis with other types of evidence such as quality of life data, data on resource use and unit prices. ${ }^{15}$ Ultimately, all parameters in these model-based economic evaluations are based on a meta-analysis or other form of synthesis of available evidence. ${ }^{15}$ Based on these data, decision-analytic models aim to reflect the course of a disease to compare the costs and consequences of competing interventions. More specifically, economic evaluations aim to inform two distinct but connected questions: ${ }^{16}$

1. whether current evidence suggests that the new treatment is cost-effective compared to current practice and;

2. whether further research into this matter would be worthwhile.

The answer to the first question depends on the differences in costs and QALYs between the treatments of interest, and the amount of money that society is willing to pay per gained QALY. This amount is referred to as the ceiling ratio. This is illustrated with an example considering a new treatment that leads to an average gain in QALYs of 0.100 and is on average $€ 10,000$ more expensive compared with current practice. These average incremental outcomes are presented (diamond) in Figure 1.1. The diagonal line represents a ceiling ratio of $€ 80,000$ per QALY gained. This is the informal ceiling ratio for a high burden of disease in the Netherlands. ${ }^{17}$ As the diamond is above this ceiling ratio, this new treatment is not deemed cost-effective and should not be reimbursed based on this information. However, average cost-effectiveness estimates, as presented in Figure 1.1, are inevitably surrounded by uncertainty. As a result, it is possible that based on current information, the 'wrong' decision is being made. Therefore, it is essential to characterize uncertainty in economic evaluations. This decision uncertainty is often incorporated in economic evaluations by reflecting the uncertainty in the input parameters of the economic evaluation representing that we do not know the exact estimates. ${ }^{15}$ The eclipse in Figure 1.2 reflects this parameter uncertainty (or sampling uncertainty) surrounding the average cost-effectiveness. As illustrated in this Figure, there is a substantial probability that the 'true' cost-effectiveness estimate for the new treatment falls 


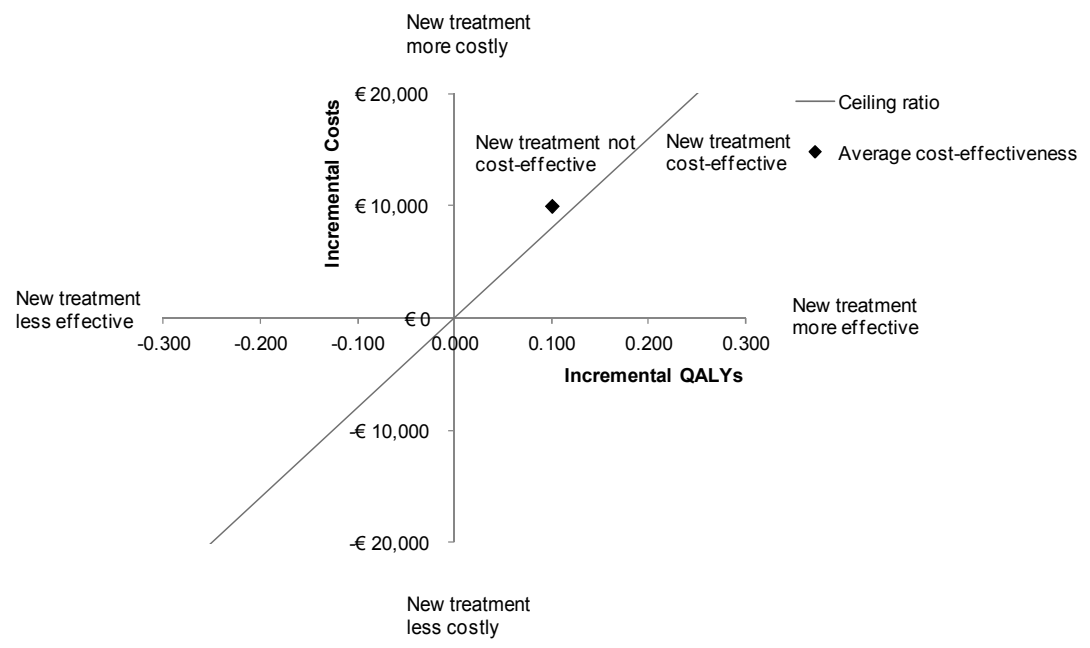

Figure 1.1: Incremental cost-effectiveness plane

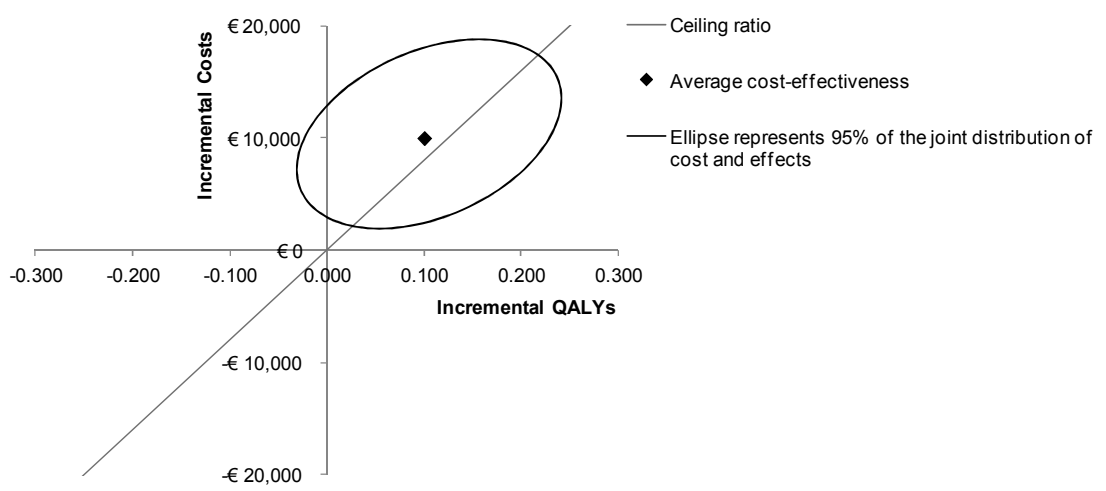

Figure 1.2: Incremental cost-effectiveness plane with estimated parameter uncertainty surrounding the average cost-effectiveness
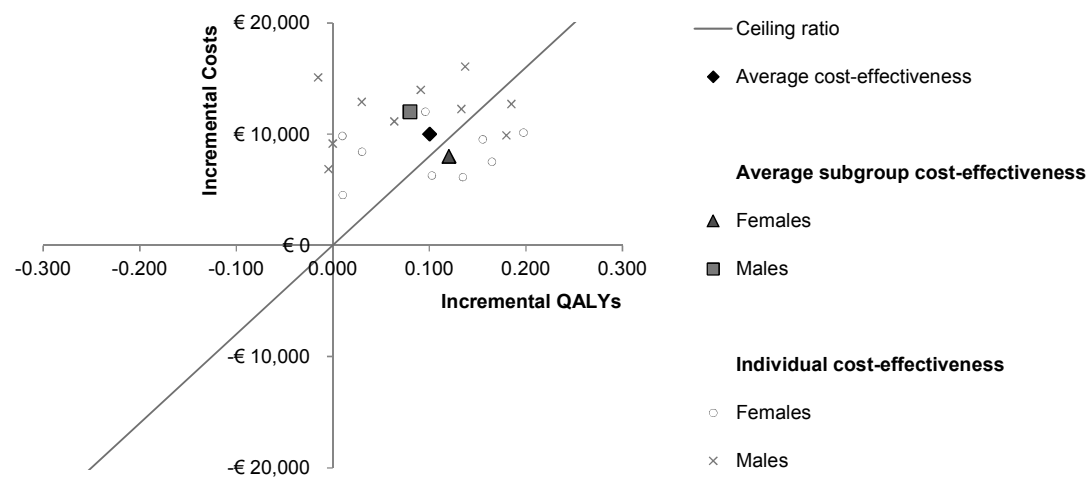

Figure 1.3: Incremental cost-effectiveness plane with individual cost-effectiveness 
below the ceiling ratio and would thus be cost-effective. This decision uncertainty is considered in the second question. The second question examines whether further research would be valuable to reduce the decision uncertainty and (re-)inform the reimbursement decision in the future. This can be quantified by using the expected value of perfect information analysis to assess the expected costs of available decision uncertainty. This is assessed by considering the certainty that the new treatment is cost-effective and the consequences if adopting the new treatment turns out to be the wrong decision (based on future information). ${ }^{15}$

The two questions mentioned above are usually informed by presenting the average cost-effectiveness for a group of patients. ${ }^{12,18}$ Although parameter uncertainty is often considered in these economic evaluations (as in Figure 1.2), variability and patient heterogeneity, are frequently neglected in average costeffectiveness estimates and subsequent reimbursement and research decisions. ${ }^{8}$ Neglecting these genuine differences between patients might mask important variations in cost-effectiveness. ${ }^{18,19}$ At the same time, as mentioned above, in clinical practice it is recognized that average outcomes may not apply to the individual patient. ${ }^{20,21}$ Thus, in contrast with individualized decisions in clinical practice, differences between individual patients are frequently neglected in economic evaluations and subsequent population-based reimbursement decisions. $^{4,8}$

\section{Personalized reimbursement decisions}

Economic evaluations are often aimed to inform reimbursement decisions. Hence, neglecting patient heterogeneity in economic evaluations impacts the allocation of resources. This may lead to inefficiency if a treatment is not costeffective for certain subgroups while it is reimbursed for all patients. For instance, in Figure 1.3, it becomes clear that the average cost-effectiveness estimate as presented in Figure 1.1 does not apply to all patients. In Figure 1.3 the dots (females) and crosses (males) represent individual patients. As shown, the effectiveness and cost-effectiveness differs per individual patient. The diamond again represents the average cost-effectiveness. Based on average cost-effectiveness estimates for all patients, the new treatment might not be reimbursed for the total group, although there are subgroups of patients for whom it is expected to be cost-effective, potentially leading to a suboptimal allocation of available resources.

Average population-based cost-effectiveness might also be suboptimal for reimbursement decisions if a treatment is not cost-effective for certain subgroups while these subgroups do receive this treatment since it is cost-effective on average. Again, this would lead to a suboptimal allocation of available re- 
sources. Therefore, a more individualized approach to the allocation of resources by providing and/or restricting treatment reimbursement to subgroups of patients (instead of one decision for all), has the potential to increase population health gains. ${ }^{18,19,22,23}$ For instance, as illustrated in Figure 1.3, the new treatment would be cost-effective on average for females (triangle below the ceiling ratio) while it is not on average cost-effective for males (triangle above the ceiling ratio). Therefore, reimbursing the new treatment for females and not for males would lead to a more optimal allocation of available resources than not reimbursing the new treatment for all patients. In this example, this would lead to improvements in terms of effectiveness (average QALY gain: 0.060) and cost-effectiveness (average net monetary benefit gain: $€ 1,570$ ).

Next to these potential gains in terms of effectiveness and/or cost-effectiveness, acknowledging patient heterogeneity is considered one of the quality criteria for good practice in economic evaluations. ${ }^{24}$ Attempts have been made to acknowledge patient heterogeneity in economic evaluations by using stratified analysis, ${ }^{22}$ regression techniques, ${ }^{25-27}$ and/or estimating the expected value of individualized care. ${ }^{28}$ Despite these incentives, patient heterogeneity is frequently neglected in current economic evaluations and subsequent policy decisions. ${ }^{8}$ This frequent neglect might in part be explained by a lack of guidance how to acknowledge patient heterogeneity, the large demand of data associated with the analyses and ethical or equity concerns when excluding patient subgroups. Firstly, guidance seems to be lacking on how and when to acknowledge patient heterogeneity. ${ }^{4,18}$ Hence, there might be an important role for pharmacoeconomic guidelines. These pharmacoeconomic guidelines provide essential guidance how economic evaluations, with the purpose to support reimbursements decision-making, should be performed within a jurisdiction. Secondly, in addition to this lack of guidance, patient heterogeneity might be neglected because of the potentially large demand of data and the computational burden associated with the analysis. Also, from a decision-making perspective, the awareness of this large data demand may lead to a priori concerns on the quality of the evidence (e.g. concerns regarding type I errors). Preferably, the analyses are based on individual patient data. ${ }^{29}$ In case these data are absent, average outcomes of comparative effectiveness research can be used. Acknowledging patient heterogeneity in economic evaluation becomes challenging if comparative effectiveness research is lacking. As comparative effectiveness research is often lacking in the early phase of new medical technologies, lacking data might impose a serious barrier to the acknowledgement of patient heterogeneity in (early) economic evaluations. Thirdly, ethical concerns may arise when acknowledging patient heterogeneity in policy decisions. For instance, ethical concerns may arise if a new treatment is effective and costeffective on average, but is not cost-effective for males. In this case, it is questionable whether it would be deemed ethical to discriminate between patients based on gender and to reimburse a treatment, which is effective for all pa- 
tients, only for females. Thus, it is open to discussion whether it is justified to withhold treatments from subgroups (e.g. males) for reasons of efficiency alone. These normative considerations and unawareness how to deal with them might lead to the neglect of patient heterogeneity.

\section{Innovative radiotherapy treatments}

Radiotherapy either alone or combined with other modalities such as chemotherapy and surgery, is an important and commonly used modality in the treatment of cancer (40-50\% of all cancer cases). ${ }^{30}$ In the past decade, innovations in radiotherapy planning and delivery have evolved rapidly. These technological advances provide unique opportunities to improve the precision and potentially also the effectiveness of radiotherapy. However, on the other hand these innovations are also associated with rising costs. ${ }^{31,32}$ As a result, the costs of radiotherapy and the total costs of oncology care are expected to accelerate while available resources are becoming restrained. ${ }^{9,10}$ Therefore, many countries require evidence on the cost-effectiveness of new treatments before a treatment is reimbursed in the statutory package of insured care. ${ }^{31}$ Evidence on the effectiveness and cost-effectiveness of innovative treatments should ideally be retrieved from a randomized controlled trial to achieve the highest grade of evidence and firm recommendations. ${ }^{33}$ Although new oncology drugs are generally evaluated in randomized controlled trials, for innovative radiotherapy technologies this is more complex and as a result comparative studies are frequently lacking. Accordingly, innovative radiotherapy technologies are often rapidly introduced into clinical practice without performing comparative studies and while considerable uncertainty still exists about its effectiveness and costeffectiveness. ${ }^{31}$ This rapid introduction is often based on the presumption that dosimetric advantages will eventually lead to better treatment outcomes, as innovative radiotherapy technologies typically result in an improved dose distribution or a more accurate dose delivery. Nevertheless, it is often uncertain to what extent the surrogate outcomes in terms of dosimetric advantages will eventually translate in better patient outcomes. Dose-response models, in terms of normal tissue complication probability (NTCP) and tumor control probability (TCP) models, can be helpful to translate these dosimetric advantages in clinical outcomes.

Another challenge is that innovative radiotherapy technologies often involve substantial investments costs in terms of equipment, quality assurance, and additional training of staff. These costs are to a large extend irreversible and thus cannot be recovered if the expected clinical benefit of the technology does not live up to its expectations. Therefore, assessing the costs of introducing novel radiotherapy technologies is more complicated than calculating the costs 
of medical drugs. ${ }^{31}$ Additionally, as mentioned above, technological advancements frequently lead to substantially higher costs (e.g. particle radiotherapy, intensity-modulated radiotherapy and positron emission tomography for treat-

ment planning). ${ }^{34}$ As a result, with regards to the challenges associated with estimating the effectiveness and costs of innovative radiotherapy technologies, it is often unknown whether these rapidly introduced treatments represent good value for money.

The technological advancements in radiotherapy are increasingly being used to strive towards individualized treatments instead of traditional treatment based on disease stage. ${ }^{30,35}$ These individualized treatment strategies require prediction models or predictive tests to estimate which treatment is optimal for which patient. It might be challenging to attain these predictive models or tests when comparative effectiveness research is frequently lacking for novel radiotherapy technologies. Additionally, in case comparative effectiveness research is available, the body of knowledge related to average outcomes is typically much larger than for subgroup outcomes, which is required to explore patient heterogeneity. ${ }^{36}$ This evidence gap hinders the implementation of individualized treatments and makes it challenging to acknowledging patient heterogeneity in costeffectiveness analysis considering innovative radiotherapy treatments.

\section{Dissertation objective}

The objective of this thesis was to examine recommendations and to explore methodology to acknowledge patient heterogeneity in economic evaluation (Figure 1.4). This objective was subdivided into two aims:

1. To examine recommendations in pharmacoeconomic guidelines on acknowledging patient heterogeneity in economic evaluations (Part I).

2. To explore innovative methodology to acknowledge patient heterogeneity in economic evaluations in case a) comparative studies are lacking and; b) if individual patient data are available (Part II).

Two case studies in the field of radiotherapy will be used to address the second aim. The first case study aims to acknowledge patient heterogeneity in economic evaluations in case comparative studies are lacking, as if often the case for novel radiotherapy technologies. ${ }^{31}$ The second case acknowledges patient heterogeneity in case comparative studies are available in terms of individual patient data from randomized controlled trials. 


\section{Dissertation outline}

Chapter 2 considers the first aim by reviewing and analyzing available national pharmacoeconomic guidelines on recommendations with regard to acknowledging patient heterogeneity in economic evaluations.

The subsequent three chapters consider aim $2 a$ by using a case study wherein proton and photon radiotherapy are compared for head and neck cancer. In Chapter 3, all available clinical and dosimetric evidence is synthesized to compare the effectiveness of proton and photon radiotherapy for head and neck cancer. Chapter 4 considers a multi-center cross-sectional study wherein the health related quality of life (HRQOL) is measured in head and neck cancer patients and the impact of treatment related toxicity on HRQOL is analysed. In Chapter 5, a model-based economic evaluation is presented to assess the cost-effectiveness in this first case study while acknowledging patient heterogeneity.

In Chapter 6, aim 2b is considered by means of a model-based economic evaluation for non-small cell lung cancer wherein modified fractionation radiotherapy schemes are compared with conventional fractionation radiotherapy while acknowledging patient heterogeneity. This economic evaluation is based on individual patient data from 10 randomized controlled trials.

Finally, in Chapter 7 a general discussion is presented which ends with concluding remarks and future perspectives. 


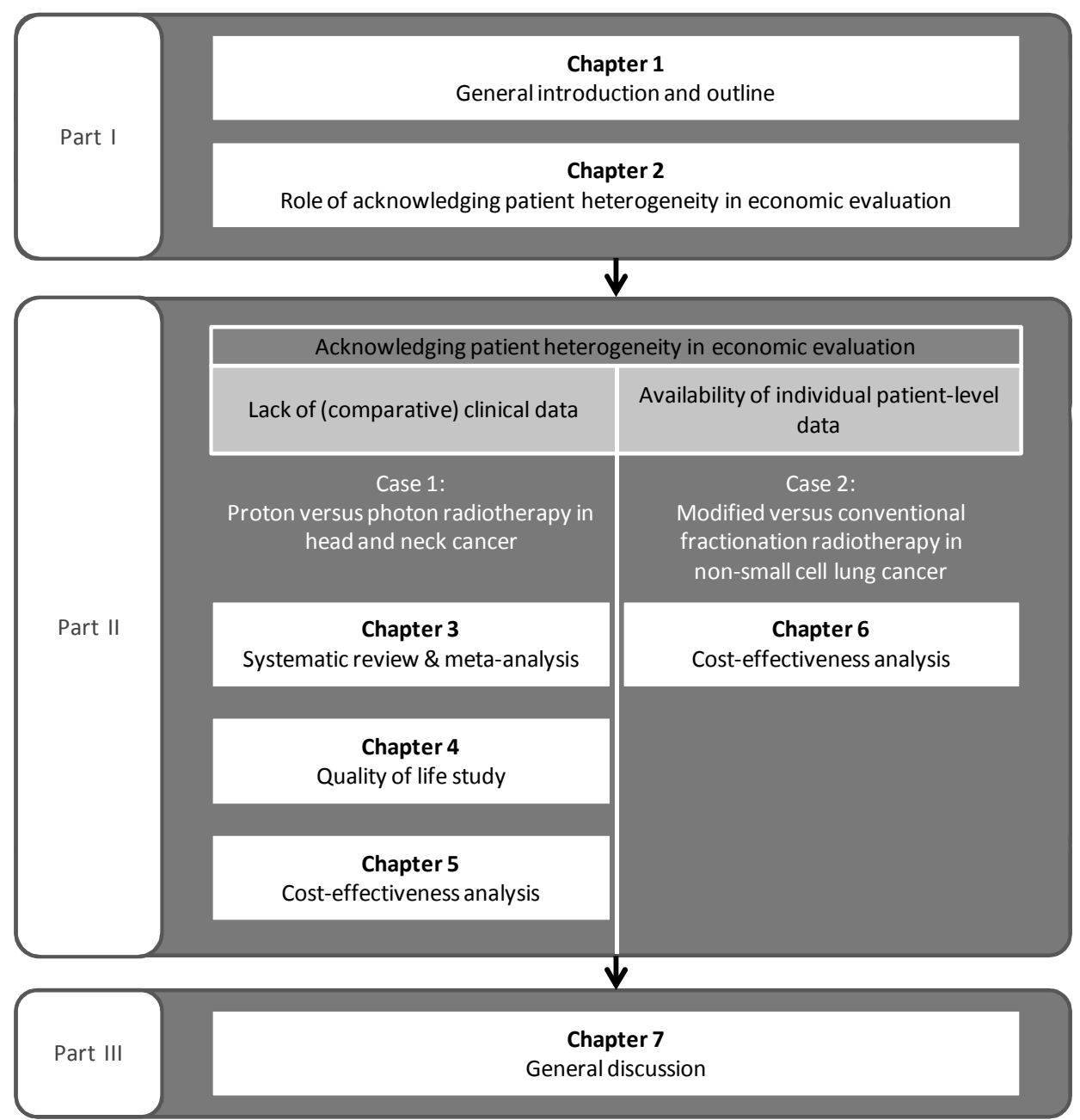

Figure 1.4: Outline of the dissertation 


\section{References}

1. Lahn BT, Ebenstein L: Let's celebrate human genetic diversity. Nature 461:726-8, 2009

2. Chopra SS: STUDENTJAMA. Preparing for personalized medicine. JAMA 291:1640, 2004

3. Bast RC, Jr., Hortobagyi GN: Individualized care for patients with cancer - a work in progress. N Engl J Med 351:2865-7, 2004

4. Grutters JP, Sculpher M, Briggs AH, et al: Acknowledging patient heterogeneity in economic evaluation: a systematic literature review. PharmacoEconomics 31:111-123, 2013

5. Evans WE, Relling MV: Moving towards individualized medicine with pharmacogenomics. Nature 429:464-8, 2004

6. Nicholson JK: Global systems biology, personalized medicine and molecular epidemiology. Mol Syst Biol 2:52, 2006

7. Schilsky RL: Personalized medicine in oncology: the future is now. Nat Rev Drug Discov 9:3636, 2010

8. Jain R, Grabner M, Onukwugha E: Sensitivity analysis in cost-effectiveness studies: from guidelines to practice. Pharmacoeconomics 29:297-314, 2011

9. Sullivan R, Peppercorn J, Sikora K, et al: Delivering affordable cancer care in high-income countries. Lancet Oncol 12:933-80, 2011

10. Meropol NJ, Schrag D, Smith TJ, et al: American Society of Clinical Oncology guidance statement: the cost of cancer care. J Clin Oncol 27:3868-74, 2009

11. INAHTA: Definitions of healthcare technology and technology assessment Accessed on 30-112012. http://inahta.episerverhotell.net/HTA/

12. Drummond MF, Sculpher MJ, Torrance GW, et al: Methods for the economic evaluation of health care programmes (ed 3rd). Oxford, Oxford University Press, 2005

13. Tolley K: What are health utilities? Accessed on January 10th, 2013. http://www.medicine.ox.ac.uk/bandolier/painres/download/whatis/Health-util.pdf

14. EuroQol Group: EuroQol--a new facility for the measurement of health-related quality of life. The EuroQol Group. Health Policy 16:199-208, 1990

15. Briggs A, Sculpher MJ, Claxton K: Decision Modelling for Health Economic Evaluation. Oxford, Oxford University Press, 2006

16. Chalkidou K, Walley T, Culyer A, et al: Evidence-informed evidence-making. J Health Serv Res Policy 13:167-73, 2008

17. Council for Public Health and Health Care Sensible and sustainable care [in Dutch]. Den Haag: Council for Public Health and Health Care 2006.

18. Sculpher M: Subgroups and heterogeneity in cost-effectiveness analysis. Pharmacoeconomics 26:799-806, 2008

19. Stevens W, Normand C: Optimisation versus certainty: understanding the issue of heterogeneity in economic evaluation. Soc Sci Med 58:315-20, 2004

20. Kattan MW, Vickers AJ: Incorporating predictions of individual patient risk in clinical trials. Urol Oncol 22:348-52, 2004

21. Kent DM, Hayward RA: Limitations of applying summary results of clinical trials to individual patients: the need for risk stratification. JAMA 298:1209-12, 2007

22. Coyle D, Buxton MJ, O'Brien BJ: Stratified cost-effectiveness analysis: a framework for establishing efficient limited use criteria. Health Econ 12:421-7, 2003

23. Zaric GS: The impact of ignoring population heterogeneity when Markov models are used in cost-effectiveness analysis. Med Decis Making 23:379-96, 2003

24. Philips Z, Ginnelly L, Sculpher M, et al: Review of guidelines for good practice in decisionanalytic modelling in health technology assessment Health Technology Assessment 8, 2004

25. Hoch JS, Briggs AH, Willan AR: Something old, something new, something borrowed, something blue: a framework for the marriage of health econometrics and cost-effectiveness analysis. Health Econ 11:415-30, 2002 
26. Nixon RM, Thompson SG: Methods for incorporating covariate adjustment, subgroup analysis and between-centre differences into cost-effectiveness evaluations. Health Econ 14:1217-29, 2005

27. Willan AR, Briggs AH, Hoch JS: Regression methods for covariate adjustment and subgroup analysis for non-censored cost-effectiveness data. Health Econ 13:461-75, 2004

28. Basu A, Meltzer D: Value of information on preference heterogeneity and individualized care. Med Decis Making 27:112-27, 2007

29. Saramago P: The Value of Further Research: The Added Value of Individual-Level Data. Value in Health 15:A492, 2012

30. Yaromina A, Krause $M$, Baumann $M$ : Individualization of cancer treatment from radiotherapy perspective. Mol Oncol 6:211-21, 2012

31. Van Loon J, Grutters J, Macbeth F: Evaluation of novel radiotherapy technologies: what evidence is needed to assess their clinical and cost effectiveness, and how should we get it? Lancet Oncol 13:e169-77, 2012

32. Bentzen SM: High-tech in radiation oncology: should there be a ceiling? Int J Radiat Oncol Biol Phys 58:320-30, 2004

33. Atkins $D$, Best $D$, Briss $P A$, et al: Grading quality of evidence and strength of recommendations. BMJ 328:1490, 2004

34. Peeters A, Grutters JP, Pijls-Johannesma M, et al: How costly is particle therapy? Cost analysis of external beam radiotherapy with carbon-ions, protons and photons. Radiother Oncol 95:45-53, 2010

35. Vordermark D: Ten years of progress in radiation oncology. BMC Cancer 11:503, 2011

36. Trusheim MR, Burgess B, Hu SX, et al: Quantifying factors for the success of stratified medicine. Nat Rev Drug Discov 10:817-33, 2011 


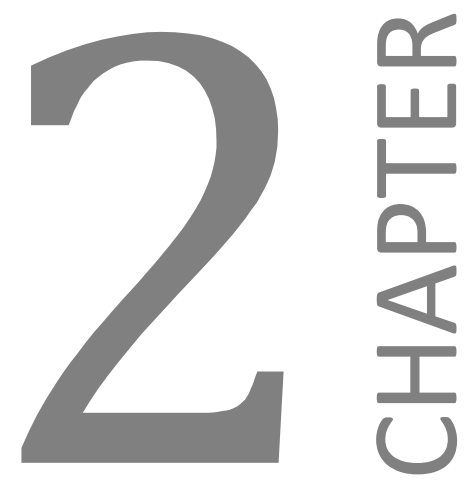

HOW SHOULD WE DEAL WITH PATIENT HETEROGENEITY IN ECONOMIC EVALUATION: A SYSTEMATIC REVIEW OF PHARMACOECONOMIC GUIDELINES

Bram Ramaekers

Manuela Joore

Janneke Grutters

Value in Health, in press 


\section{Abstract}

Objective: To review and analyse recommendations from national pharmacoeconomic guidelines with regard to acknowledging patient heterogeneity in economic evaluations.

Methods: National pharmacoeconomic guidelines were obtained through the ISPOR website. Guidance was extracted using a developed data extraction sheet. Extracted data were divided into subcategories based on consensus meetings.

Results: Of the 26 included guidelines, $20(77 \%)$ advised to identify patient heterogeneity. Most guidelines $(77 \%)$ provided general methodological advice to acknowledge patient heterogeneity; including justifications for distinguishing subgroups $(65 \%)$, prespecification of subgroups $(42 \%)$ or methodology to acknowledge patient heterogeneity $(77 \%)$. Stratified analysis of costeffectiveness was most commonly advised (20 guidelines; $77 \%$ ), however, guidance on the specific application of methods was scarce (nine guidelines; $34 \%$ ) and generally limited if provided. Guidance to present patient heterogeneity was provided by 15 guidelines $(58 \%)$, most prominently to describe the definition (31\%) and justification (31\%) of subgroups.

Conclusions: The majority of national pharmacoeconomic guidelines provide guidance on acknowledging patient heterogeneity in economic evaluations. However, since guidance is mostly not specific, its usefulness is limited. This may reflect that the importance of acknowledging patient heterogeneity is usually recognized while there is a lack of consensus on specific methods to acknowledge patient heterogeneity. We advise the further development of national pharmacoeconomic guidelines to provide specific guidance on the identification of patient heterogeneity, methods to acknowledge it and presenting the results. We present a checklist which can assist in formulating these recommendations. This could facilitate the systematic and transparent handling of patient heterogeneity in economic evaluations worldwide.

Keywords: Patient heterogeneity; National pharmacoeconomic guideline; Economic evaluation; Systematic review 


\section{Introduction}

Considering the rapidly increasing healthcare costs and the finite amount of available resources, the criteria to grant reimbursement to new treatments have become more restricted. These reimbursement decisions are often made for groups of patients. However, a more individualized approach for the allocation of available resources, i.e. providing treatment reimbursement for subgroups of patients, has the potential to increase population health gains. ${ }^{1-4}$ Acknowledging patient heterogeneity in reimbursement decisions may lead to more efficient healthcare if these reimbursement decisions are based on cost-effectiveness. ${ }^{5}$ As economic evaluations are frequently used to estimate cost-effectiveness and support reimbursement decision-making, ${ }^{6}$ it is essential that patient heterogeneity is incorporated in economic evaluations. Although there is consensus on its importance, ${ }^{7}$ patient heterogeneity is frequently neglected in economic evaluations. $^{8}$

Patient heterogeneity might be neglected since subgroup policy sometimes is controversial due to ethical concerns. This may lead to equity constraints, where the use of certain characteristics is considered unacceptable to determine which subgroups have access to a technology. The acknowledgement of patient heterogeneity in economic evaluations seems also hampered by a lack of clarity on when and how this should be done., ${ }^{2,9}$ In this respect, there is an important role for national pharmacoeconomic guidelines. National pharmacoeconomic guidelines provide essential guidance how economic evaluations, with the purpose to support reimbursements decision-making, should be performed within a jurisdiction. The objective of this study was therefore to review and analyse recommendations from national pharmacoeconomic guidelines with regard to acknowledging patient heterogeneity in economic evaluations.

\section{Methods}

\section{Definition of patient heterogeneity}

Patient heterogeneity was defined as the part of the natural variation between patients (variability) that can be attributed to characteristics of those patients $^{6,9,10}$. This was differentiated from treatment variability (differences in the nature of the treatment), differences between geographical regions that may impact cost-effectiveness and statistical heterogeneity. These concepts relate more to the generalizability of cost-effectiveness results ${ }^{9,11}$ and variation in outcomes between studies (e.g. included in a meta-analysis) and are beyond the scope of this review. 
Characteristics that potentially explain patient heterogeneity include demographics (e.g. age, gender, income), preferences (e.g. attitude, beliefs, risk tolerance) and/or clinical characteristics (e.g. disease severity, disease history, genetic profile). ${ }^{9}$ These sources of patient heterogeneity may impact on different input parameters used in an economic evaluation: baseline risks, relative treatment effects, health state utility and resource utilization. ${ }^{9}$ Differences in unit costs are more likely a result of differences between geographical regions and are thus not considered in this review. ${ }^{9,12}$

\section{Search strategy and data extraction}

Consistent with previous reviews of national pharmacoeconomic guidelines, ${ }^{12,13}$ national pharmacoeconomic guidelines were obtained through the link provided on the International Society for Pharmacoeconomics and Outcomes Research (ISPOR) website (accessed February 22th, 2013) ${ }^{14}$ and retrieved from the website of the guideline agencies. This was done to ensure that the most recent versions were retrieved. The ISPOR website was considered a reliable and valid source as the overview of national pharmacoeconomic guidelines is based on contacts with experts from approximately 60 countries from around the world. ${ }^{12}$ Guidelines were included if they were available in English. To systematically extract relevant guidance, we used a data extraction sheet (Appendix 2.1) containing the following categories:

1. Acknowledgement of patient heterogeneity:

whether guidelines advised to identify patient heterogeneity and whether a distinction is made between different inputs of an economic evaluation: 1) baseline risk, 2) relative treatment effect, 3) health state utility and 4) resource utilization.

2. Methodology to acknowledge patient heterogeneity:

whether guidelines advised methodology to acknowledge patient heterogeneity. This contains guidance whether to justify for acknowledging patient heterogeneity (including equity constraints), guidance whether to prespecify potential sources of patient heterogeneity, general methods and the specific application of methods to acknowledge patient heterogeneity.

3. Presentation of patient heterogeneity:

whether guidelines advised what should be presented when acknowledging patient heterogeneity.

Data were extracted and categorized (into the abovementioned categories) by one author (BR). Extracted data from all guidelines were divided into subcategories. If the classification of guidance was ambiguous, it was independently judged by the other authors (JG and MJ). Possible discrepancies were discussed to reach consensus. 


\section{Results}

In total 33 guidelines were retrieved. Seven guidelines were excluded since they were not available in English. ${ }^{15-21}$ This accumulated to 26 included guidelines, published between 1997 and 2012. ${ }^{22-47}$

\section{Acknowledgement of patient heterogeneity}

Most guidelines (20;77\%) advise to identify patient heterogeneity in general. ${ }^{22-}$ ${ }^{41}$ Thirteen guidelines $(50 \%)$ explicitly consider it relevant to identify the impact of patient heterogeneity on effects in general (irrespective of whether it has an impact on the baseline risk and/or treatment effect). ${ }^{22-34}$ Seven guidelines $(27 \%)$ specify this into differences in baseline risk and treatment effect and consider them both as relevant. ${ }^{22-28}$ Additionally, four guidelines consider it relevant to reflect the impact of patient heterogeneity on health state utility. ${ }^{24,26,29,31}$ Nine guidelines $(35 \%)$ consider differences in resource utilization as relevant input to acknowledge patient heterogeneity. ${ }^{23-26,28,30,31,33,34}$ None of the guidelines advise not to identify patient heterogeneity in any of these four key inputs of an economic evaluation.

\section{Methodology to acknowledge patient heterogeneity in economic evaluations}

Methodological guidance on acknowledging patient heterogeneity is provided by 20 guidelines $(77 \%)^{22-41}$

Arguments to justify acknowledging patient heterogeneity in economic evaluations

Arguments to justify acknowledging patient heterogeneity are required by 17 guidelines (65\%). ${ }^{22-32,34-39}$ Only the England and Wales guideline ${ }^{25}$ lists equity constraints (Table 2.1). Instead of neglecting subgroups based on a particular equity point of view, the Canadian guideline ${ }^{24}$ proposes to calculate the opportunity costs of equity concerns using the framework proposed by Coyle et al. ${ }^{1}$ These opportunity costs can be interpreted as the costs of neglecting subgroups based on grounds of equity. Hence, this framework aims to inform the tradeoff between equity and efficiency. ${ }^{1}$ Additionally, the German guideline states that only subgroups should be addressed for which an additional benefit or lesser harm was established. ${ }^{40}$

\section{Specification of potential sources of patient heterogeneity}

Eight guidelines $(31 \%),{ }^{22,24-27,30,32,37}$ advise to prespecify potential sources of patient heterogeneity (Table 2.1). The French guideline considers posthoc multivariate analysis acceptable to explore patient heterogeneity. ${ }^{29}$ Posthoc 
analysis is allowed under certain conditions by eight guidelines (31\%): only for differences in costs, ${ }^{23}$ with (strong) justification ${ }^{24,28}$ and/or if interpreted as explorative, ${ }^{23}$ with caution ${ }^{22,26,27}$ or hypothesis generating. ${ }^{24,32}$

Table 2.1: Overview of advice on methodology from guidelines to acknowledge patient heterogeneity

\begin{tabular}{|c|c|c|c|}
\hline \multicolumn{3}{|c|}{ Guidelines } & \multirow{2}{*}{$\begin{array}{l}\text { Countries } \\
A U, B C, B E, C A, E W, \\
F I, F R, D E, H U, I E, I T, \\
N Z, N O, P L, P T, S L \text {, } \\
Z A, E S, S E, U S\end{array}$} \\
\hline & & & \\
\hline A & $\begin{array}{l}\text { Guidelines that advised to justify for acknowledging } \\
\text { patient heterogeneity }\end{array}$ & $17(65 \%)$ & $\begin{array}{l}A U, B C, B E, C A, E W, \\
F I, F R, H U, I E, N Z, N O \text {, } \\
P L, P T, S L, Z A, S E, U S\end{array}$ \\
\hline
\end{tabular}

Specific guidance:

- If plausible based on (pre)clinical evidence/pharmacokinetics

$11(42 \%) \quad A U, B C, B E, E W, F R$,

If plausible based on a priori expectations of costeffectiveness

$7(27 \%) \quad$ BC, BE, EW, HU, IE,

If biologically plausible

$5(19 \%) \quad A U, E W, I E, N Z, S L$

- If statistically plausible

- If patients for whom it is most (cost-)effective can be targeted/if relevant for decision

$4(15 \%) \quad A U, F R, N Z, Z A$

$4(15 \%) \quad$ NZ, NO, ZA, US

- If relevant for distributive aspects/if patient groups likely to be disadvantaged can be targeted

- If informative for value based pricing

- If subgroups are within the approved indication

- If not solely based on: 1) individual utilities for health states and patient preferences, or 2) differential treatment costs for individuals according to their social characteristics

B Guidelines that advised to prespecify potential sources of patient heterogeneity

Specific guidance:

- Prespecify subgroups

- Interpret posthoc analysis as explorative/as hypothesis generating/ with caution

$2(8 \%) \quad C A, F R$

$1(4 \%) \quad \mathrm{FI}$

$1(4 \%) \quad \mathrm{ZA}$

$1(4 \%) \quad E W$

- Adhoc data mining should be avoided

- Posthoc analysis are only allowed with (strong) justification

- Posthoc analysis only for differences in costs

- Posthoc multivariate analysis is acceptable to explore patient heterogeneity

$8(31 \%) \quad$ AU, CA, EW, HU, IE, NZ, PT, ZA

$6(23 \%) \quad \mathrm{AU}, \mathrm{BE}, \mathrm{CA}, \mathrm{IE}, \mathrm{NZ}, \mathrm{PT}$

$3(12 \%) \quad B E, E W, S L$

$2(8 \%) \quad \mathrm{CA}, \mathrm{SL}$

$1(4 \%) \quad B E$

$1(4 \%) \quad F R$

Country codes according to the ISO 3166-1 Alpha-2 if applicable: Australia ${ }^{22}=\mathrm{AU}$, Baltics (Latvia, Estonia and Lithuania $)^{34}=\mathrm{BC}^{*}$, Belgium ${ }^{23}=\mathrm{BE}$, Canada ${ }^{24}=\mathrm{CA}$, England and Wales ${ }^{25}=\mathrm{EW}^{*}$, Finland $^{35}=\mathrm{FI}$, France ${ }^{29}=\mathrm{FR}$, Germany ${ }^{40}=\mathrm{DE}$, Hungary ${ }^{30}=\mathrm{HU}$, Ireland ${ }^{26}=\mathrm{IE}$, Israel ${ }^{41}=\mathrm{IL}_{\text {, New }}$ Zealand $^{27}=$ NZ, Norway ${ }^{36}=$ NO, Poland ${ }^{31}=$ PL, Portugal ${ }^{32}=$ PT, Scotland ${ }^{28}=$ SL $^{*}$, South Africa ${ }^{37}=$ ZA, Spain $^{33}=$ ES, Sweden ${ }^{38}=$ SE, United States ${ }^{39}=$ US

*Unofficial codes 
Table 2.1 (continued): Overview of advice on methodology from guidelines to acknowledge patient heterogeneity

\begin{tabular}{|c|c|c|c|c|}
\hline & \multicolumn{3}{|l|}{ Guidelines } & \multirow{2}{*}{$\begin{array}{l}\text { Countries } \\
A U, B C, B E, C A, E W, \\
F I, F R, D E, H U, I E, I T \\
N Z, N O, P L, P T, S L \\
Z A, E S, S E, U S\end{array}$} \\
\hline C & $\begin{array}{l}\text { Guidelines that advised how to acknowledge patient } \\
\text { heterogeneity in economic evaluations }\end{array}$ & $20(77 \%)$ & & \\
\hline & Suggested methods: & & & \\
\hline & - Stratified analysis & & $20(77 \%)$ & $\begin{array}{l}\text { AU, BC, BE, CA, EW, } \\
\text { FI, FR, DE, HU, IE, IT, } \\
\text { NZ, NO, PL, PT, SL, } \\
\text { ZA, ES, SE, US }\end{array}$ \\
\hline & Decision analytic modelling & & $3(12 \%)$ & FR, DE, SL \\
\hline & - Sensitivity analysis/scenario analysis & & $3(12 \%)$ & $\mathrm{AU}, \mathrm{CA}, \mathrm{EW}$ \\
\hline & Specific advice on the application of methods: & & & \\
\hline & $\begin{array}{l}\text { - Statistical precision of subgroups estimates should be } \\
\text { reflected in the analysis of parameter uncertainty }\end{array}$ & & $3(12 \%)$ & $\mathrm{CA}, \mathrm{EW}, \mathrm{SL}$ \\
\hline & $\begin{array}{l}\text { - Calculate the impact of variability in baseline risk by } \\
\text { multiplying the expected baseline risk across patient } \\
\text { subgroups by the overall relative treatment effect } \\
\text { (established in the whole population) }\end{array}$ & & $3(12 \%)$ & $A U, B E, N Z$ \\
\hline & $\begin{array}{l}\text { - Meta-regression to determine whether a treatment } \\
\text { effect varies across patient groups }\end{array}$ & & $2(8 \%)$ & $\mathrm{AU}, \mathrm{SL}$ \\
\hline & $\begin{array}{l}\text { Calculate the impact of variability in absolute treatment } \\
\text { effect by applying the estimated relative treatment } \\
\text { effect for the subgroups to the expected baseline risk } \\
\text { for the subgroups }\end{array}$ & & $1(4 \%)$ & AU \\
\hline & - Discrete event simulation & & $1(4 \%)$ & DE \\
\hline & $\begin{array}{l}\text { - Sensitivity analysis for equity concerns and subgroup } \\
\text { thresholds }\end{array}$ & & $1(4 \%)$ & AU \\
\hline & - Scenario analysis for treatment continuation rules & & $1(4 \%)$ & EW \\
\hline & $\begin{array}{l}\text { - Multivariate analysis to evaluate treatment effective- } \\
\text { ness depending on patient characteristics }\end{array}$ & & $1(4 \%)$ & FR \\
\hline & - Separate models for separate subgroups & & $1(4 \%)$ & $\mathrm{CA}$ \\
\hline & $\begin{array}{l}\text { Equivalent data for stratified analysis as for the whole } \\
\text { group }\end{array}$ & & $1(4 \%)$ & $\mathrm{Fl}$ \\
\hline & $\begin{array}{l}\text { - Examine whether the relative risk is constant over } \\
\text { different baseline risk }\end{array}$ & & $1(4 \%)$ & SL \\
\hline & $\begin{array}{l}\text { - Explore the possibility that differences between groups } \\
\text { emerge by chance }\end{array}$ & & $1(4 \%)$ & $\mathrm{EW}$ \\
\hline
\end{tabular}

Country codes according to the ISO 3166-1 Alpha-2 if applicable: Australia ${ }^{22}=\mathrm{AU}$, Baltics (Latvia, Estonia and Lithuania) ${ }^{34}=\mathrm{BC}^{*}$, Belgium $^{23}=\mathrm{BE}$, Canada ${ }^{24}=\mathrm{CA}$, England and Wales ${ }^{25}=\mathrm{EW}^{*}$, Finland $^{35}=\mathrm{FI}$, France ${ }^{29}=\mathrm{FR}$, Germany ${ }^{40}=\mathrm{DE}$, Hungary $\mathrm{H}^{30}=\mathrm{HU}$, Ireland ${ }^{26}=\mathrm{IE}$, Israel ${ }^{41}=\mathrm{IL}$, New

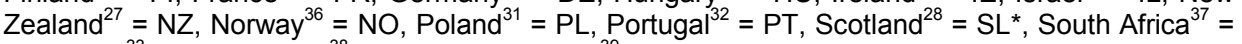
ZA, Spain ${ }^{33}=$ ES, Sweden ${ }^{38}=$ SE, United States ${ }^{39}=$ US

*Unofficial codes

\section{How to acknowledge patient heterogeneity}

Most guidelines $(20 ; 77 \%)$ provide general advice how to acknowledge patient heterogeneity. ${ }^{22-41}$ Stratified analysis is the most commonly advised method. ${ }^{22-41}$ The French, German and Scottish guidelines generally advise the use of decision analytic modelling. ${ }^{28,29,40}$ Further, sensitivity and/or scenario analyses are 
advised by the guidelines from Australia, Canada and England and Wales. ${ }^{2,24,25}$ However, most guidelines merely provide general guidance; details on the specific application of methods is provided by a minority $(35 \%)$ of guidelines (Table 2.1). ${ }^{22-25,27-29,35,40}$

The German guideline advises a specific form of modelling: discrete event simulation (DES). They argue that patient heterogeneity can be incorporated into DES as each patient can be modeled with its unique characteristics. ${ }^{40}$ The Canadian guideline advises stratified cost-effectiveness analysis according to the framework by Coyle et al. ${ }^{1}$ to calculate the potential efficiency gain of subgroup policy. The Australian guideline argues that meta-regression is preferred above stratified analysis since it allows to examine multiple covariates simultaneously. ${ }^{22}$ The French guideline advises the use of multivariate analysis to evaluate treatment effectiveness depending on patient characteristics. ${ }^{29}$ Additionally, the Australian guideline advises that the absolute treatment effect can be calculated in case of differences in baseline risk and/or treatment effect by applying the estimated relative treatment effect for the subgroups to the expected baseline risk for the subgroups. ${ }^{22}$ The Belgian and New-Zealand guidelines advise to multiply the expected baseline risk across patient subgroups by the overall relative treatment effect (assuming a constant relative treatment effect for all subgroups). ${ }^{23,27}$

The Australian guideline recommends performing sensitivity analysis for equity concerns that affect the cost-effectiveness and subgroup thresholds for continuous variables such as age. ${ }^{22}$ Additionally, the England and Wales guideline advises scenario analysis for treatment continuation rules. ${ }^{25}$ These rules can be used to adjust treatment plans based on patient heterogeneity that is revealed over time (e.g. differences in treatment response). The guideline provides guidance on the specification of treatment continuation rules focusing on the feasibility, robustness and equity of implementing these rules. ${ }^{25}$ Finally, the England and Wales guideline advises to explore the possibility that differences between subgroups emerge by chance, especially in case numerous subgroups are reported. ${ }^{25}$

\section{Presenting patient heterogeneity}

Guidance on presenting patient heterogeneity is provided by 15 guidelines (58\%) (Table 2.2). ${ }^{22-29,31,33,34,37-40}$ For the methods section, the most common advice was to describe the definition ${ }^{22-25,27,28,37,40}$ and justification of subgroups. $^{22,23,25-28,31,34}$ Cost-effectiveness estimates should be presented separately for each subgroup in the results section according to seven guidelines $(27 \%){ }^{24-26,29,33,39,40}$ Presenting the implications of subgroup policy on distributional aspects is recommended by six guidelines $(23 \%)$. $^{22,24,26,29,39,40}$ This includes highlighting unmet needs of certain disadvantaged groups. The United States guideline advises to weigh subgroup outcomes against moral values. ${ }^{39}$ 
Detailed tables to present the assessment of treatment effects across subgroups are provided by the Australian guideline. ${ }^{22}$ For the discussion section, it was recommended to highlight biomarkers/diagnostic tests necessary to identify relevant subgroups, ${ }^{22,39}$ whether subgroup results lead to different conclusions than the overall trial results, ${ }^{22}$ the credibility of the claim to use subgroups ${ }^{22,39}$ and the appropriate use of the intervention. ${ }^{24}$

Table 2.2: Overview of advice on the presentation of patient heterogeneity from guidelines

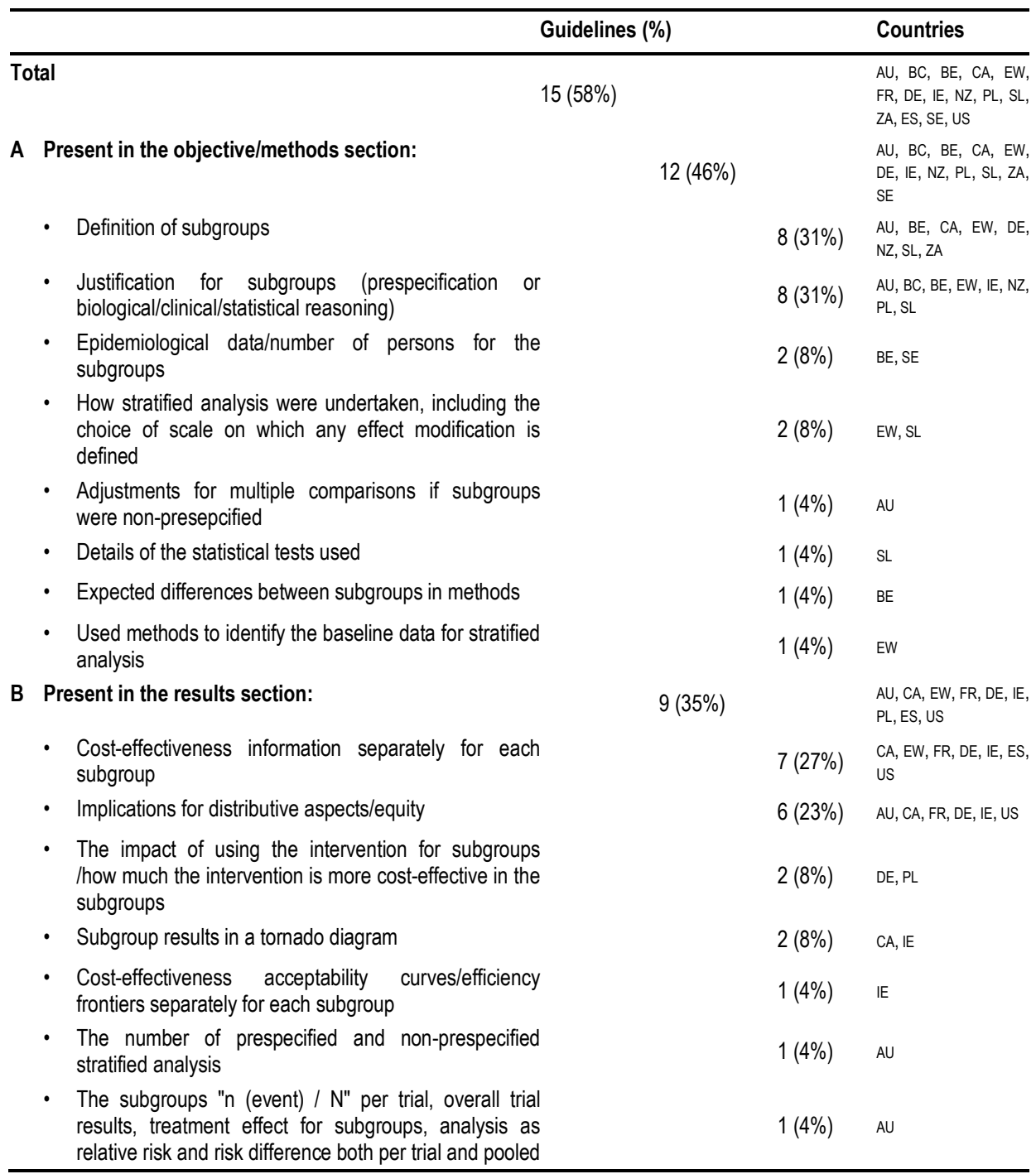

Country codes according to the ISO 3166-1 Alpha-2 if applicable: Australia ${ }^{22}=\mathrm{AU}$, Baltics (Latvia, Estonia and Lithuania $)^{34}=\mathrm{BC}^{*}$, Belgium ${ }^{23}=\mathrm{BE}$, Canada $^{24}=\mathrm{CA}$, England and Wales ${ }^{25}=\mathrm{EW}^{*}$, France $^{29}=\mathrm{FR}$, Germany ${ }^{40}=\mathrm{DE}$, Ireland ${ }^{26}=\mathrm{IE}$, New Zealand ${ }^{27}=\mathrm{NZ}$, Poland ${ }^{31}=\mathrm{PL}$, Scotland ${ }^{28}=$ $\mathrm{SL}^{*}$, South Africa ${ }^{37}=\mathrm{ZA}$, Spain ${ }^{33}=\mathrm{ES}$, Sweden $^{38}=\mathrm{SE}$, United State ${ }^{39}=$ US

*Unofficial codes 
Table 2.2 (continued): Overview of advice on the presentation of patient heterogeneity from guidelines

\begin{tabular}{|c|c|c|c|c|}
\hline & \multicolumn{3}{|c|}{ Guidelines (\%) } & \multirow{2}{*}{ Countries } \\
\hline C & Present in the discussion section: & $3(12 \%)$ & & \\
\hline & $\begin{array}{l}\text { Discuss the claim to justify the use of subgroups (e.g. } \\
\text { evidence or pharmacological, biological and clinical } \\
\text { plausibility for the variation in (cost-)effectiveness). }\end{array}$ & & $2(8 \%)$ & $\mathrm{AU}, \mathrm{US}$ \\
\hline & $\begin{array}{l}\text { Discuss the biomarkers or other diagnostics (e.g. } \\
\text { validity, reliability and feasibility for clinical practice) } \\
\text { necessary to identify patient subgroups }\end{array}$ & & $2(8 \%)$ & $\mathrm{AU}, \mathrm{US}$ \\
\hline & $\begin{array}{l}\text { Discuss whether subgroup results lead to different } \\
\text { conclusions than the primary overall trial results. }\end{array}$ & & $1(4 \%)$ & AU \\
\hline & - Discuss the appropriate use of the intervention. & & $1(4 \%)$ & $\mathrm{CA}$ \\
\hline
\end{tabular}

Country codes according to the ISO 3166-1 Alpha-2 if applicable: Australia ${ }^{22}=A U$, Baltics (Latvia, Estonia and Lithuania) $)^{34}=\mathrm{BC}^{*}$, Belgium $^{23}=\mathrm{BE}$, Canada ${ }^{24}=\mathrm{CA}$, England and Wales ${ }^{25}=\mathrm{EW}^{*}$, France $^{29}=$ FR, Germany ${ }^{40}=$ DE, Ireland ${ }^{26}=I E$, New Zealand ${ }^{27}=$ NZ, Poland ${ }^{31}=$ PL, Scotland ${ }^{28}=$ $\mathrm{SL}^{*}$, South Africa ${ }^{37}=\mathrm{ZA}$, Spain $^{33}=\mathrm{ES}$, Sweden $^{38}=\mathrm{SE}$, United States ${ }^{39}=\mathrm{US}$

*Unofficial codes

\section{Discussion}

We reviewed guidance from national pharmacoeconomic guidelines with regard to acknowledging patient heterogeneity in economic evaluations. Although the majority of guidelines considered it relevant to acknowledge patient heterogeneity, only few specified specifically which inputs of an economic evaluation are relevant for this purpose. Consistently, most guidelines provide general guidance on how to acknowledge patient heterogeneity (mostly stratified analysis). However, specific and in depth guidance on applying for instance stratified analysis or other methods was scarce and generally limited if provided. Also, guidance was "heterogeneous" between guidelines. This might reflect differences between healthcare systems or jurisdictions. For example, if costeffectiveness is important for reimbursement decisions, guidelines might be more specific and directive. This can be illustrated by England and Wales, where reimbursement decisions are based on cost-effectiveness outcomes, and which accordingly provides one of the most specific guidelines. Overall, our review revealed that the importance of acknowledging patient heterogeneity is usually recognized, while there is a lack of consensus and specific guidance on acknowledging and presenting patient heterogeneity.

One study limitation was that we excluded seven guidelines which were not written in English. Translations of guidelines from South America and Asia would be helpful to obtain a complete overview. Nevertheless, with 26 included guidelines, this review provides a comprehensive and probably representative overview of guidance on handling patient heterogeneity in economic evaluation. Also, the review was restricted to guidelines available on the ISPOR website. 
As a result, some guidelines may have been missed in the review. However, when considering that a large part of the national pharmacoeconomic guidelines is published in the "grey literature", a systematic search strategy in for instance PubMed would most likely not lead to a representative and complete overview of guidelines. Alternatively, using personal knowledge and contacts to identify national pharmacoeconomic guidelines would potentially lead to selection bias, i.e. pharmacoeconomic guidelines from well-known guideline authorities (e.g. NICE) would be more likely to be included. In contrast, consulting the ISPOR website to identify national pharmacoeconomic guidelines can be regarded as a reproducible and thus systematic method to identify these guidelines and provide a representative overview of guidance. To our opinion, there is no better alternative to identify national pharmacoeconomic guidelines and consulting the ISPOR website can thus be regarded as the best available method for this purpose. Furthermore, there will certainly be subjectivity in our assessment of for example what is considered guidance and what is not. Hence, some of our evaluations may be criticized. However, by developing a data extraction sheet and organizing consensus meetings, we have put all efforts to keep the assessments as systematic and objective as possible. Moreover, despite some potentially subjective judgments, this study aimed to facilitate informed discussions and advance current practice of economic evaluations. Finally, our review was restricted to pharmacoeconomic guidelines, whereas guidelines on the clinical effectiveness/benefit assessment might provide recommendations on handling patient heterogeneity for differences in relative treatment effectiveness. This restriction is to our opinion appropriate since economic evaluations have a broader perspective; they consider consequences on an absolute scale and include additional input parameters as baseline risk, health state utility and resource utilization. Additionally, health economic researchers will probably consult and adhere to the national pharmacoeconomic guideline.

In addition to the limitations in the presented review, current literature and guidelines might sometimes be confusing concerning the handling of patient heterogeneity. The German guideline ${ }^{40}$ uses patient heterogeneity as an argument for patient-level simulation (DES). This argument is to our opinion incorrect. Patient-level simulation is not necessarily required to acknowledge patient heterogeneity (see for example $e^{48,49}$ ). Subgroups can for instance be modeled in cohort models by letting them start in different health states. ${ }^{50}$ Patient-level simulation is a useful alternative if this becomes too complex. ${ }^{9}$

Although it inevitably takes time before new concepts are incorporated in guidelines, it is obvious that more clear and specific guidance would be useful. Ideally, guidelines would clearly state situations in which patient heterogeneity is considered irrelevant for decision-making in their jurisdiction (e.g. using equity constraints as the guideline from England and Wales ${ }^{25}$ ). This enables researchers to focus on subgroups that are potentially useful for decision-making. The framework presented by Grutters et al. might be valuable to systematically 
explore which sources and inputs of patient heterogeneity are deemed (ir)relevant. ${ }^{9}$ Additionally, individual preferences are a special source of patient heterogeneity. Preference subgroups can be acknowledged by using individual preferences. ${ }^{5,51}$ However, it has been debated whether individual preferences should be incorporated in population reimbursement decisions. ${ }^{52-55}$ Subgroups based on individual utilities may be inconsistent with the idea that societal welfare should be determined by aggregating the preferences of society. ${ }^{56,57}$ However, as proposed by Sculpher and Gafni, it is possible to acknowledge diversity in the preferences of individuals while maintaining the use of utility values of the general public. ${ }^{58}$ Guidelines should clarify how to deal with differences in individual preferences. The guideline from England and Wales for instance argues against subgroups solely based on individual utilities.

Most guidelines consider patient heterogeneity that is known at the start of treatment. Additionally, the England and Wales guideline provides guidance to construct treatment continuation rules based on patient heterogeneity revealed over time. ${ }^{25}$ Treatment continuation rules (e.g. based on treatment response) can be used to adjust treatment plans after treatment start. However, it might be complex to attain feasible treatment continuation rules and inform actual reimbursement decisions based on patient heterogeneity revealed over time. Therefore, guidelines should clarify, as the England and Wales guideline ${ }^{25}$ how patient heterogeneity revealed over time should be handled.

It is recommended that guidelines are as specific as possible when stratified analysis should be undertaken (e.g. what justification is required and in which circumstances posthoc analyses are allowed). This potentially prevents that subgroups are only analysed if overall cost-effectiveness is hard to show. Additionally, the possibility of false positives due to random noise in data might caution researchers or policy makers to use subgroups in their analysis or decision-making. ${ }^{9}$ However, these fears exist mainly from an epidemiological perspective. ${ }^{3}$ From a health economic perspective, the statistical significance of subgroups is irrelevant; ${ }^{59}$ rather its value for reimbursement decisions is relevant. Certainly, this does not mean that uncertainty is irrelevant ${ }^{59,60}$ and data dredging should always be avoided. However, the role of stratified analysis in economic evaluations should to our opinion be considered based on its value for policy purposes (i.e. the forgone health benefits if subgroups are neglected), rather than from an epidemiological perspective. ${ }^{61}$

The finding that none of the guidelines provided specific guidance how to select influential subgroups and determine subgroup thresholds or the optimal number of subgroups for continuous variables (e.g. to determine age groups) might reflect that there is no consensus on this topic. Thus, the selection of patient subgroups, particularly in case multiple (continuous) variables are considered simultaneously, may require further methodological research, as currently performed by, among others, Saramago ${ }^{62}$ and Espinoza et al $^{63}$. Nevertheless efforts have been made in this direction, Willan, Briggs and Hoch demonstrated 
that regression techniques can be used within the net benefit framework to explore and select statistically significant patient characteristics to define subgroups. ${ }^{61,64}$ Furthermore, Basu and Meltzer proposed a framework to estimate the expected value of individualized care ${ }^{5}$. By selecting potentially influential variables based on the parameter specific value of individualized care, this framework could be an alternative to the regression technique described above., ${ }^{5,65}$ The selected variables can subsequently be used in stratified analyses by estimating the benefits of providing different treatments to different subgroups and hence calculating the potential value of subgroup policy. ${ }^{1}$ This would give decision-makers the opportunity to judge when the costs of equity constraints are too high. ${ }^{66}$ In decision analytic modelling, stratified analyses can be performed by using different input values for different subgroups. This can be implemented by linking input parameters to patient characteristics or by adding health states and letting different subgroups start out in different health states. ${ }^{4,6,9,50}$

Additionally, it might be useful to provide guidance what should be presented to support potential reimbursement decisions based on subgroup outcomes. This includes for instance to describe and justify subgroups, provide guidance on specific tables/figures and highlighting issues as the appropriate use of the intervention and the feasibility of subgroup policy. These recommendations require a high level of prescription in guidelines, while many are currently not this directive. It is open to debate whether decision-makers wish to have such prescriptive guidelines, or prefer to leave this to the integrity of researchers. In our opinion, decision-makers should clearly state which results are needed for their appraisal and how these results should be achieved in the assessment, in order to support uniformity. Taking into account the differences between jurisdictions (e.g.in terms of legislation and normative judgments), it was deemed impossible to formulate guidance that would be appropriate for all national pharmacoeconomic guidelines worldwide. Therefore, we present a checklist in Table 2.3 to assist national guideline authorities to formulate comprehensive recommendations with regards to acknowledging patient heterogeneity in economic evaluations on the topics identified in this review. Obviously, researchers are allowed to deviate from guideline recommendations if appropriately justified. However, especially when considering a normative subject where subgroups are potentially included or excluded from treatment reimbursement, guidelines should in our opinion be specific and directive.

In conclusion, the majority of national pharmacoeconomic guidelines provide guidance on acknowledging patient heterogeneity in economic evaluations. However, since specific guidance is often lacking, its usefulness is limited. This may reflect that the importance of acknowledging patient heterogeneity is usually recognized while there is a lack of consensus on specific requirements and methodology to acknowledge and present patient heterogeneity in economic evaluations. We advise the further development of national pharmacoeco- 
nomic guidelines to provide specific guidance in each of the categories: the identification of patient heterogeneity, methods to acknowledge patient heterogeneity, and presenting the results when acknowledging patient heterogeneity. This could facilitate the systematic and transparent handling of patient heterogeneity in economic evaluations worldwide.

Table 2.3: Checklist to formulate guidance on acknowledging patient heterogeneity in economic evaluation

Acknowledgement of patient heterogeneity

1 In economic evaluations patient heterogeneity

$\square$ Should not be reflected

$\square$ Should be reflected

2 When acknowledging patient heterogeneity, the following inputs of an economic evaluation should be considered*

$\square$ Baseline risk

$\square$ Relative treatment effect

$\square$ Health state utility

$\square$ Resource utilization

3 The following sources of patient heterogeneity should be considered*

$\square$ Demographics

$\square$ Preferences

$\square$ Clinical characteristics

Methodology to acknowledge patient heterogeneity

4 In order to analyse different sources of patient heterogeneity*

$\square$ No justification is required

$\square \quad$ This should be justified based on biological plausibility

$\square \quad$ This should be justified based on (pre)clinical evidence/pharmacokinetics

$\square \quad$ This should be justified based on statistical plausibility

$\square$ This should be justified based on ...

5 Subgroups that are a priori not considered relevant for decision-making are those based solely on the following sources of patient heterogeneity

$\square \quad$ No subgroups are a priori considered irrelevant.

$\square$ Subgroups based on the following sources are a priori considered irrelevant: ...

6 Patient heterogeneity should be explored based on

$\square$ Prespecified subgroups and posthoc analysis should be avoided

$\square$ Prespecified subgroups and posthoc analysis should only be used to generate hypotheses or to ...

$\square$ Either posthoc analysis or prespecified subgroups.

7 The following methods are suggested to analyse patient heterogeneity *

$\square$ Stratified analysis

$\square$ Decision analytic modelling

$\square$ Sensitivity or scenario analysis

$\square$ Expected value of individualized care

$\square$ Regression analysis

*Possible to select multiple options 
Table 2.3 (continued): Checklist to formulate guidance on acknowledging patient heterogeneity in economic evaluation

8 These methods should be applied in order to*

$\square$ Incorporate and analyse sources of patient heterogeneity if ...

$\square$ Incorporate and analyse variability in baseline risk and/or treatment effect through ...

$\square$ Determine subgroup thresholds and the number of subgroups in case of continuous variables through ...

$\square$ Reflect the precision of subgroup estimates.

$\square$ Handle patient heterogeneity revealed over time.

Presenting patient heterogeneity

9 To support subgroup policymaking the following information should be presented*

$\square$ Clear definition and justification of subgroups

$\square$ Details on data used to produce subgroup estimates

$\square$ Details on statistical analyses

$\square$ Stratified cost-effectiveness outcomes

$\square$ Tornado diagram

$\square$ Separate cost-effectiveness acceptability curves

$\square$ Implications for distributive aspects/equity

$\square$ Validity, reliability and feasibility of biomarkers of diagnostics necessary to identify subgroups

$\square$ Appropriate use of the intervention

${ }^{*}$ Possible to select multiple options

\section{Acknowledgement}

This study was supported by an unrestricted research grant (No. 152002021) from the Dutch Organization of Health Research and Development (ZonMw). ZonMw had no influence on the study design, data analyses, data interpretation, manuscript writing or the decision to submit the manuscript for publication. The advisory board of the ZonMw project, consisting of Dr. Albert Boer, Prof. dr. Andrew Briggs, Dr. Math Candel, Prof. dr. Dirk De Ruysscher, Prof. dr. Mark Sculpher, Prof. dr. Johan Severens, and Dr. James Stahl, is greatly acknowledged for their valuable contribution to the project. 


\section{References}

1. Coyle D, Buxton MJ, O'Brien BJ: Stratified cost-effectiveness analysis: a framework for establishing efficient limited use criteria. Health Econ 12:421-7, 2003

2. Sculpher M: Subgroups and heterogeneity in cost-effectiveness analysis. Pharmacoeconomics 26:799-806, 2008

3. Stevens W, Normand C: Optimisation versus certainty: understanding the issue of heterogeneity in economic evaluation. Soc Sci Med 58:315-20, 2004

4. Zaric GS: The impact of ignoring population heterogeneity when Markov models are used in cost-effectiveness analysis. Med Decis Making 23:379-96, 2003

5. Basu A, Meltzer D: Value of information on preference heterogeneity and individualized care. Med Decis Making 27:112-27, 2007

6. Briggs A, Sculpher MJ, Claxton K: Decision Modelling for Health Economic Evaluation. Oxford, Oxford University Press, 2006

7. Philips Z, Ginnelly L, Sculpher M, et al: Review of guidelines for good practice in decisionanalytic modelling in health technology assessment Health Technology Assessment 8, 2004:1158

8. Jain R, Grabner M, Onukwugha E: Sensitivity analysis in cost-effectiveness studies: from guidelines to practice. Pharmacoeconomics 29:297-314, 2011

9. Grutters JP, Sculpher M, Briggs $\mathrm{AH}$, et al: Acknowledging patient heterogeneity in economic evaluation: a systematic literature review. PharmacoEconomics 31:111-123, 2013

10. Briggs $A H$, Weinstein MC, Fenwick EA, et al: Model parameter estimation and uncertainty: a report of the ISPOR-SMDM Modeling Good Research Practices Task Force--6. Value Health 15:835-42, 2012

11. Sculpher MJ, Pang FS, Manca A, et al: Generalisability in economic evaluation studies in healthcare: a review and case studies. Health Technol Assess 8:iii-iv, 1-192, 2004

12. Barbieri $M$, Drummond $M$, Rutten $F$, et al: What do international pharmacoeconomic guidelines say about economic data transferability? Value Health 13:1028-37, 2010

13. Knies S, Severens JL, Ament AJ, et al: The transferability of valuing lost productivity across jurisdictions. differences between national pharmacoeconomic guidelines. Value Health 13:51927,2010

14. International Society for Pharmacoeconomics and Outcomes Research (ISPOR): Pharmacoeconomic Guidelines Around The World Accessed on February 22th. http://www.ispor.org/PEguidelines/index.asp

15. González AMG. Methodological Guidelines for Health Economic Evaluation. Cuba: Ministry of Public Health, 2003.

16. De Mello Vianna CM, Caetano R. Methodological Guidelines: Economic Evaluation of Health Technologies. Brazil: Ministry of Health, 2009.

17. China Guidelines for Pharmacoeconomic Evaluations. China: PharmacoEconomics China, 2011.

18. Guidelines for Economic Appraisal Studies for Updating the Health Sector's National Formulary in Mexico. Mexico: National Institute of Public Health, 2008.

19. Guidelines for Economic Evaluation of Health Care Interventions. Slovakia: Ministry of Health Slovak Republic, 2008.

20. Pharmaceutical Economic Evaluation Guidelines. South Korea: Health Insurance Review Agency, 2006.

21. Guidelines of Methodological Standards for Pharmacoeconomic Evaluations. Taiwan: Taiwan Society for Pharmacoeconomics and Outcomes Research, 2006.

22. Guidelines for preparing submissions to the Pharmaceutical Benefits Advisory Committee. Australia: Pharmaceutical Benefits Advisory Committee, 2008.

23. Cleemput I, Van Wilder $P$, Vrijens $F$, et al. Guidelines for pharmacoeconomic evaluations in Belgium. Belgium: Federaal Kenniscentrum voor de Gezondheidszorg, 2008. 
24. Guidelines for the economic evaluation of health technologies. Canada: Canadian Agency for Drugs and Technologies in Health, 2006.

25. Guide to the methods of technology appraisal. England and Wales: National Institute for Health and Clinical Excellence, 2008.

26. Guidelines for the Economic Evaluation of Health Technologies in Ireland. Ireland: Health Information and Quality Authority, 2010.

27. Prescription for pharmacoeconomic analysis. New Zealand: Pharmaceutical Management Agency, 2007.

28. Guidance to Manufacturers for completion of New Product Assessment Form. Scotland: Scottish Medicines Consortium, 2007.

29. French guidelines for the economic evaluation of health care technologies. France: Collège des Économistes de la Santé, 2004.

30. Szende Á, Mogyorósy Z, Muszbek N, et al: Methodological guidelines for conducting economic evaluation of healthcare interventions in Hungary: a Hungarian proposal for methodology standards. Eur J Health Econom 3:196-206, 2002

31. Guidelines for conducting Health Technology Assessment. Poland: Agency for Health Technology Assessment, 2009.

32. Da Silva EA, Pinto CG, Sampaio C, et al. Guidelines for Economic Drug Evaluation Studies. Portugal: National Authority of Medicines and Health Products, 1998.

33. López-Bastida J, Oliva J, Antoñanzas F, et al: Spanish recommendations on economic evaluation of health technologies. Eur J Health Econom 11:513-20, 2010

34. Behmane D, Lambot K, Irs A, et al. Baltic Guideline for Economic Evaluation of Pharmaceuticals (pharmacoeconomic analysis). The Baltics: Baltic health authorities, 2002.

35. Guidelines for preparing a health economic evaluation [unofficial translation]. Finland: Ministry of Social Affairs and Health, 2009.

36. Guidelines on how to conduct pharmacoeconomic analyses. Norway: The Norwegian Medicines Agency, 2012

37. Regulations relating to a transparent pricing system for medicines and scheduled substances: (draft guidelines for pharmacoeconomic submissions). South Africa: Department of Health, 2010.

38. General guidelines for economic evaluations from the Pharmaceutical Benefits Board. Sweden: The Pharmaceutical Benefits Board, 2003.

39. The AMCP Format for Formulary Submissions Version 3.0. United States: Academy of Managed Care Pharmacy, 2009.

40. General Methods for the Assessment of the Relation of Benefits to Costs. Germany: Institute for Quality and Efficiency in Health Care, 2009.

41. Guidelines for the submission of a request to include a pharmaceutical product in the national list of health services. Israel: Ministry of Health Pharmaceutical Administration, 2010.

42. Walter E, Zehetmayr S. Guidelines on Health Economic Evaluation. Austria: Institute for Pharmaeconomic Research, 2006.

43. Guidelines for pharmacoeconomic research. The Netherlands: The Health Care Insurance Board, 2006.

44. Teerawattananon Y, Chaikledkaew U: Thai Health Technology Assessment Guideline Development. J Med Assoc Thai 91:S11-S15, 2008

45. Capri S, Ceci A, Terranova L, et al: Guidelines for economic evaluations in Italy: recommendations from the Italian group of pharmacoeconomic studies. Drug Information Journal 35:189-201, 2001

46. Alban A, Gyldmark M, Pedersen AV, et al: The Danish approach to standards for economic evaluation methodologies. Pharmacoeconomics 12:627-36, 1997

47. Avksentieva MV, Antonova NV, Arutyunov GP, et al. Procedure for clinical and economic evaluation of drug lists that are submitted for reimbursement coverage from public health care budget. Russia: Research Center for Clinical and Economic Evaluation and Pharmacoeconomics of the Russian State Medical University, 2010. 
48. Briggs A, Mihaylova B, Sculpher $M$, et al: Cost effectiveness of perindopril in reducing cardiovascular events in patients with stable coronary artery disease using data from the EUROPA study. Heart 93:1081-6, 2007

49. Ramaekers BL, Grutters JP, Pijls-Johannesma M, et al. Protons in Head-and-Neck Cancer: Bridging the Gap of Evidence. Int J Radiat Oncol Biol Phys 85:1282-8, 2013

50. Brennan A, Chick SE, Davies R: A taxonomy of model structures for economic evaluation of health technologies. Health Econ 15:1295-310, 2006

51. Bala MV, Zarkin GA: Pharmacogenomics and the evolution of healthcare : is it time for costeffectiveness analysis at the individual level? Pharmacoeconomics 22:495-8, 2004

52. Brazier JE, Dixon S, Ratcliffe J: The role of patient preferences in cost-effectiveness analysis: a conflict of values? Pharmacoeconomics 27:705-12, 2009

53. Bryan S, Dolan P: Discrete choice experiments in health economics. For better or for worse? Eur J Health Econ 5:199-202, 2004

54. Dowie J: Towards the equitably efficient and transparently decidable use of public funds in the deep blue millennium. Health Econ 7:93-103, 1998

55. Sculpher M, Gafni A, Watt I: Shared treatment decision making in a collectively funded health care system: possible conflicts and some potential solutions. Soc Sci Med 54:1369-77, 2002

56. Robinson A, Parkin D: Recognising diversity in public preferences: the use of preference subgroups in cost-effectiveness analysis. A response to Sculpher and Gafni. Health Econ 11:64951; discussion 653-4, 2002

57. Sculpher M, Gafni A: Recognizing diversity in public preferences: the use of preference subgroups in cost-effectiveness analysis. Author's reply. Health Econ 11:653-654, 2002

58. Sculpher M, Gafni A: Recognizing diversity in public preferences: the use of preference subgroups in cost-effectiveness analysis. Health Econ 10:317-24, 2001

59. Claxton K: The irrelevance of inference: a decision-making approach to the stochastic evaluation of health care technologies. J Health Econ 18:341-64, 1999

60. Griffin SC, Claxton KP, Palmer SJ, et al: Dangerous omissions: the consequences of ignoring decision uncertainty. Health Econ 20:212-24, 2011

61. Willan AR, Briggs AH, Hoch JS: Regression methods for covariate adjustment and subgroup analysis for non-censored cost-effectiveness data. Health Econ 13:461-75, 2004

62. Saramago P: The Value of Further Research: The Added Value of Individual-Level Data. Value in Health 15:A492, 2012

63. Espinoza M, Manca A, Claxton $K$, et al: The value of heterogeneity for cost-effectiveness subgroup analysis: Theoretical framework and application. Value in Health 15:A241, 2012

64. Hoch JS, Briggs AH, Willan AR: Something old, something new, something borrowed, something blue: a framework for the marriage of health econometrics and cost-effectiveness analysis. Health Econ 11:415-30, 2002

65. Van Gestel A, Grutters J, Schouten J, et al: The role of the expected value of individualized care in cost-effectiveness analyses and decision making. Value Health 15:13-21, 2012

66. Sculpher M. Briefing paper for methods review workshop on identifying sub-groups and exploring heterogeneity. National Institute for Health and Clinical Excellence (NICE), 2007. 


\section{Appendix 2.1: Data extraction sheet}

\section{General}

Country:

Year:

Source or web link:

Developed by:

Type of Guideline: Pharmacoeconomic / Submission

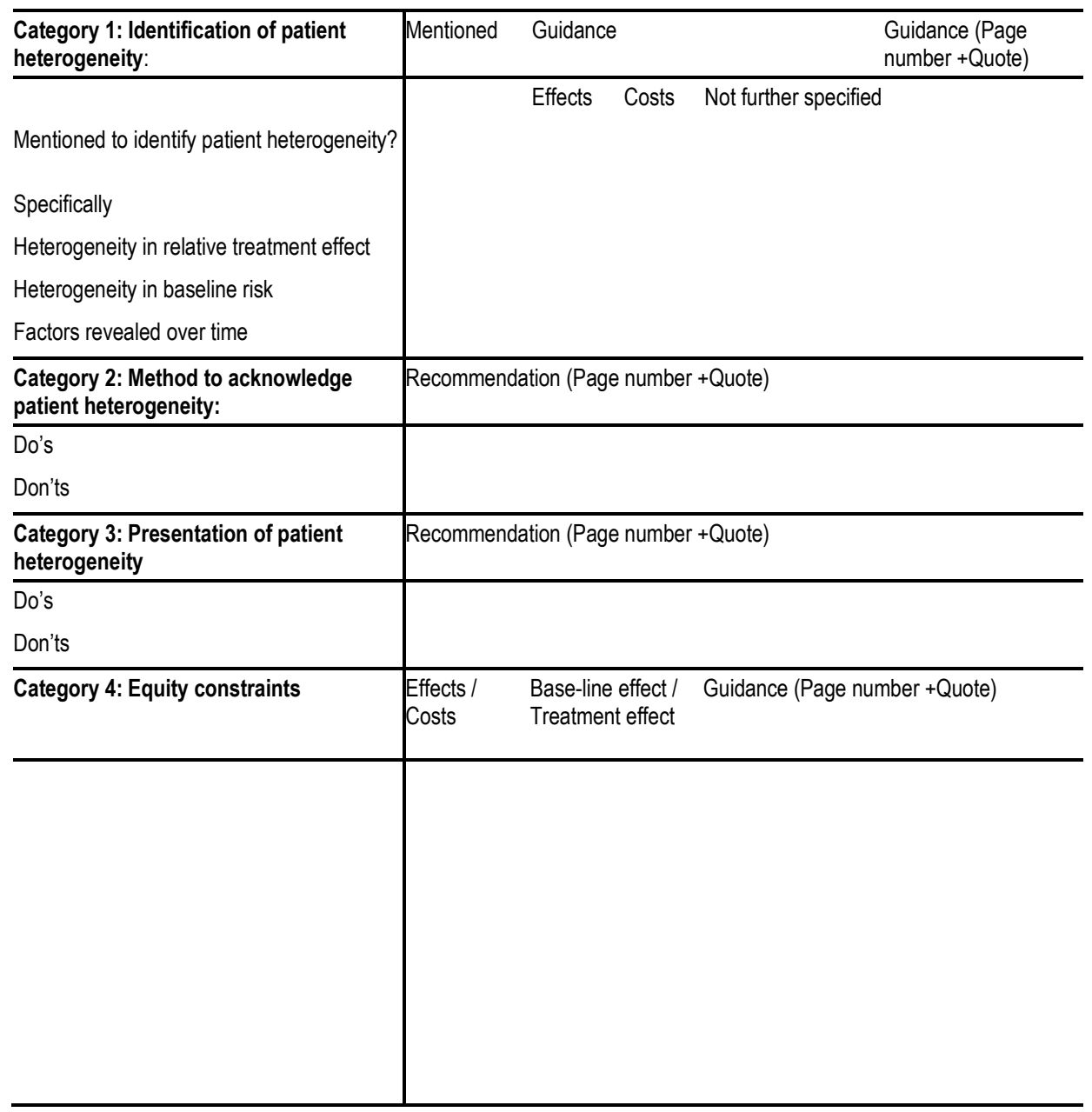

Ns $=$ Not specified

\section{Other remarks:}





\section{PART II}

Acknowledging heterogeneity in health technology assessment in radiotherapy

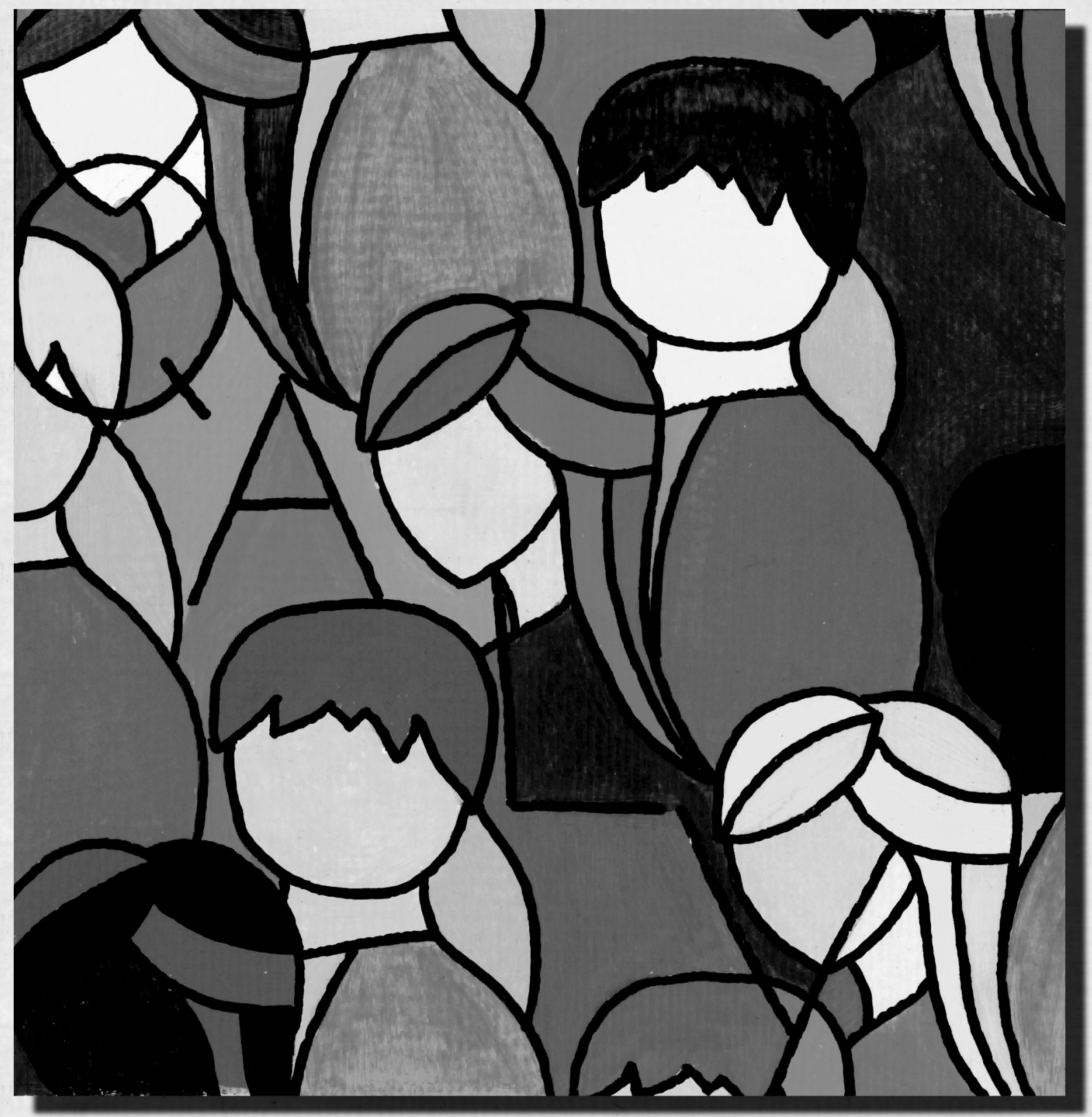





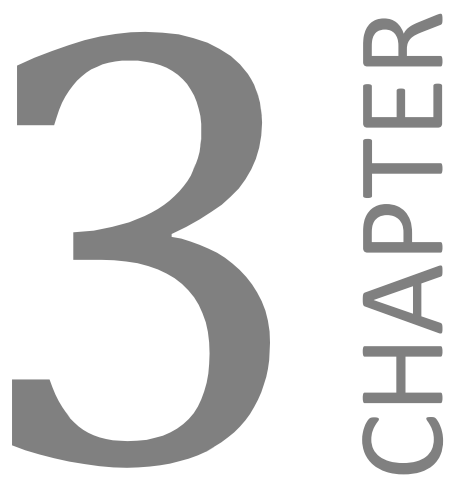

SYSTEMATIC REVIEW AND META-ANALYSIS OF RADIOTHERAPY IN VARIOUS HEAD AND NECK CANCERS: COMPARING PHOTONS, CARBON-IONS AND PROTONS

\author{
Bram Ramaekers \\ Madelon Pijls-Johannesma \\ Manuela Joore \\ Piet Van Den Ende \\ Johannes Langendijk \\ Philippe Lambin \\ Alfons Kessels \\ Janneke Grutters
}




\section{Abstract}

Objective: To synthesize and compare available evidence considering the effectiveness of carbon-ion, proton and photon radiotherapy for head and neck cancer.

Methods: A systematic review and meta-analyses were performed to retrieve evidence on tumor control, survival and late treatment toxicity for carbon-ion, proton and the best available photon radiotherapy.

Results: In total 86 observational studies ( 74 photon, 5 carbon-ion and 7 proton) and 8 comparative in-silico studies were included. For mucosal malignant melanomas, 5-year survival was significantly higher after carbon-ion therapy compared to conventional photon therapy ( $44 \%$ vs. $25 \%$; P-value 0.007 ). Also, 5 -year local control after proton therapy was significantly higher for paranasal and sinonasal cancer compared to intensity-modulated photon therapy $(88 \%$ vs. $66 \%$; P-value 0.035 ). No other statistically significant differences were observed. Although poorly reported, toxicity tended to be less frequent in carbonion and proton studies compared to photons.

In-silico studies showed a lower dose to the organs at risk, independently of the tumor site.

Conclusions: For carbon-ion therapy, the increased survival in mucosal malignant melanomas might suggest an advantage in treating relatively radioresistant tumors. Except for paranasal and sinonasal cancer, survival and tumor control for proton therapy were generally similar to the best available photon radiotherapy. In agreement with included in-silico studies, limited available clinical data indicates that toxicity tends to be lower for proton compared to photon radiotherapy.

Since the overall quantity and quality of data regarding carbon-ion and proton therapy is poor, we recommend the construction of an international particle therapy register to facilitate definitive comparisons.

Keywords: Radiotherapy; Photon; IMRT; Particle; Carbon-ion; Proton; Head and neck cancer; Meta-analysis; Systematic review 


\section{Introduction}

Head and neck cancer (HNC) covers a heterogeneous group of cancers, which includes paranasal and sinonasal cancer and cancer of the salivary gland, lip, oral cavity, pharynx and larynx. When considering cancer of the oral cavity, pharynx and larynx in Europe, the annual incidence is approximately 147,500. This represents $4.6 \%$ of all cancer cases, accounting for 63,400 deaths. ${ }^{1}$

Treatment options for HNC consist of radiotherapy, surgery, chemotherapy or a combination of these modalities. There is a growing body of evidence suggesting that more aggressive treatment regimes, such as altered fractionation schedules for radiotherapy or (concomitant) chemo-radiation improve tumor control and survival. ${ }^{2-4}$ Recent meta-analyses showed absolute improvements in 5 -year survival of $3 \%$ for altered fractionation versus conventional fractionation and of $5 \%$ for chemotherapy versus no chemotherapy. ${ }^{3,4}$ However, mainly due to the close proximity of critical organs and the often large irradiation fields, the improved outcomes in these aggressive treatment regimens come at the cost of increased treatment toxicity. Late toxicities (including xerostomia and dysphagia) affect a substantial proportion of HNC patients and negatively impacts patients' functional outcomes and quality of life. ${ }^{5-9}$

Particle therapy using carbon-ions or protons is currently gaining importance worldwide. ${ }^{10}$ Compared with photons, the in-depth dose distribution of particles allows a more accurate dose administration, resulting in an increased therapeutic ratio. Based on these physical characteristics, it is supposed that less normal tissue will be irradiated compared with photon radiation, presumably resulting in less treatment related toxicity. ${ }^{11,12}$ On the other hand, the increased therapeutic ratio possibly permits dose escalation to the tumor, resulting in a higher tumor control probability. Next to these physical advantages, carbon-ion radiotherapy comprises an increased radiobiological efficacy, especially in the Bragg peak. Hence, to decrease treatment related toxicity and potentially further improve outcome, particle therapy could be a valuable technology in the treatment of HNC. Nevertheless, several reviews indicated that based on clinical evidence it remains unclear, mainly because of the absence of randomized trials, whether particle therapy is superior over radiotherapy with photons in HNC. ${ }^{13-20}$ This makes it difficult to decide whether particle therapy should be implemented as a (routine) treatment in clinical practice. ${ }^{21}$ However, decisions need to be made. In addition to the available clinical evidence, comparative in-silico studies, which analyse computer-predicted dose parameters in radiotherapy treatment planning for different radiation techniques, offer an alternative approach to estimate the potential gain of particle therapy. ${ }^{15,22}$ Our aim was therefore to synthesize all available evidence, from clinical studies as well as comparative in-silico studies. This resulted in the following objectives 1) to review and synthesize available clinical evidence considering the effectiveness (i.e. tumor control, survival and 
late treatment toxicity) of carbon-ion and proton radiotherapy compared with the best available photon radiation in patients receiving radiotherapy treatment for $\mathrm{HNC}$ and, 2) to review available comparative in-silico studies comparing carbon-ion and proton radiotherapy with photon therapy in HNC.

\section{Methods}

\section{Search Strategy and Selection Criteria}

Systematic literature searches were conducted to identify available clinical particle and photon studies. A first search query was constructed to identify studies using carbon-ions or protons in HNC. Included photon studies were identified using a second (intensity modulated radiotherapy) and third (conventional radiotherapy) search query. The PICO (patient, intervention, comparison and outcome) framework ${ }^{23}$ was used to structure all three search queries in PubMed. MeSH terms as well as free text words were used. The keywords were 'head and neck cancer' AND ('proton radiotherapy' OR 'carbon-ion radiotherapy' OR 'intensity modulated radiotherapy' OR 'radiotherapy') AND ('survival' OR 'adverse effects'). The full search strategy is reported in Appendix 3.1.

All search queries were updated until February 2010. In addition, manual searches of reference lists were performed to complement all three search queries. For particle therapy, supplementary manual searches of conference proceedings were performed.

Clinical studies were included if they were published after 1990, written in English, examined carbon-ions, protons or photons, examined treatment of primary tumors, examined radical treatment (defined as a treatment with the intent to obtain loco-regional disease control) and had a patient sample $\geq 10$. For photon therapy, if available, only studies using intensity modulated radiotherapy (IMRT) were included. If no IMRT studies were available, studies administering conventional photon therapy were included. Further, photon studies were only included if tumor control or survival data was reported. In order to collect all available evidence on particle therapy, particle studies were also included if they report toxicity data only. Occasionally data were reported in multiple publications. In case of overlap in data and if both studies reported the required data, only the study with the largest sample size was included.

To identify in-silico studies comparing particle therapy and photon therapy in HNC, a fourth search strategy was constructed. This search query was structured as described above for clinical studies. The search query was equal to the first search query, except for the keyword 'radiotherapy planning' instead of ('survival' OR 'adverse effects') (Appendix 3.1).

In-silico studies were included if they were published after 1990, written in English, performed a direct comparison of particle therapy (carbon-ions or 
protons) with photon therapy, examined treatment of primary tumors, reported dose to the organs at risk and had a patient sample $\geq 5$.

\section{Data Extraction}

General characteristics were extracted from clinical studies: first author, year of publication, treatment type, sample size, dose per fraction, number of fractions, total treatment dose, study population (age, tumor stage, location and histology, percentage of operated patients, percentage patients of receiving chemotherapy), late treatment toxicity, local control, disease free survival and overall survival outcomes at 2- to 5-year. Since HNC represents a heterogeneous patient group with different treatment modalities and prognosis, the comparisons of clinical studies were categorized according to location or histology. The subgroups were nasopharyngeal cancer, oropharyngeal cancer, paranasal and sinonasal cancer, mucosal malignant melanomas and adenoid cystic carcinomas. Because radiotherapy toxicity is location specific, different types of toxicities were examined for different subgroups. Xerostomia and dysphagia were considered in nasopharyngeal and oropharyngeal cancer, visual toxicity in paranasal and sinonasal cancer, visual, skin and mucosal toxicity in mucosal malignant melanomas and all reported toxicity in adenoid cystic carcinomas. Toxicity data were extracted per type and grade independently of the classification system.

The following characteristics were extracted for included in-silico studies: first author, year of publication, treatment types, sample size, tumor location and/or histology, dose parameters of target volume (coverage, conformity index ${ }^{24}$ and inhomogeneity) and dose to organs at risk (dose to the optic nerves for paranasal sinus carcinomas and for all other sites dose to the parotid glands and pharyngeal constrictors). These organs at risk were selected since they are associated with visual impairment, ${ }^{25}$ xerostomia ${ }^{26}$ and dysphagia. ${ }^{27,28}$

All data were independently extracted by two reviewers (BR and MPJ), if necessary data were extracted from figures. Discrepancies were solved in consensus meetings with all authors.

\section{Statistical Analysis}

If tumor control or survival outcomes were available per subgroup for at least 2 comparators, data from clinical studies were pooled for the longest available follow-up period. Pooling was performed by means of a random effect metaregression analysis. If corresponding standard errors (se) were not reported in included studies, we calculated them using the standard method for proportions. ${ }^{29}$ If patients were censored, we used the Peto method. ${ }^{30,31}$ In case the number of censored patients was not reported, we assumed the maximum 
percentage of censored patients reported in the included studies in the subgroup concerned.

Through relating the outcomes (e.g. survival) to potentially confounding variables (e.g. differences between the included studies in the percentage of patients receiving chemotherapy) in the meta-regression analyses, we explored the effect of these covariates on the outcome. ${ }^{32}$ We did this by firstly calculating the overall mean for these covariates among all included studies in the subgroup concerned. Secondly, we calculated for each study separately how much the covariate deviated from the overall mean. Finally, this deviation from the overall mean was included in the meta-regression model as an independent covariate. This was done separately for each subgroup for the following covariates: the proportion of patients having T4 tumors, N3 tumors, stage IV tumors, the proportion of patients receiving chemotherapy, the proportion of operated patients and publication year. Only covariates which significantly influenced the outcomes were included in the final meta-regression models.

For comparative in-silico studies, results were reviewed only and no statistical analyses were performed.

All analyses were performed in STATA 9.1 and a P-value $\leq 0.05$ was considered statistically significant. Further, this meta-analysis was performed according to proposed guidelines. ${ }^{33}$

\section{Results}

The three search strategies considering clinical studies identified 86 eligible observational studies: 5 studies using carbon-ion therapy, 7 studies using proton therapy and 74 studies using photon therapy. The fourth search strategy identified 8 eligible comparative in-silico studies (Appendix 3.2). Included and excluded particle studies and in-silico studies were reported in Appendix 3.3. The characteristics of the clinical studies pooled in the meta-analyses were reported in Appendix 3.4. Further, results of the meta-analyses are presented in Figure 3.1 and Table 3.1. The 8 in-silico studies were reviewed separately from the clinical studies.

\section{Head and neck cancer}

Three studies on particle therapy (two carbon-ion and one proton) ${ }^{34-36}$ and eight studies on IMRT ${ }^{37-44}$ reported on a mixed group (multiple sub-sites) of HNC patients. Local control (LC) rates at 2- and 5 year were $80-85 \%$ and $60-70 \%$ for carbon-ion therapy, $87 \%$ and $74 \%$ for proton therapy and 2 -year LC ranged between $76-100 \%$ for IMRT. Disease free survival (DFS) estimates at 2- and 5year were $56 \%$ and $29 \%$ for proton therapy and $81-90 \%$ at 2 -year for IMRT. Overall survival (OS) at 2- and 5-year were respectively $74 \%$ and $33-48 \%$ for 
carbon-ion therapy; $69 \%$ and $44 \%$ for proton therapy and $83-90 \%$ at 2 -year for IMRT. Due to heterogeneity within and between these studies, these results deemed largely not comparable and outcomes were therefore not pooled in a meta-analysis.

\section{Nasopharyngeal carcinoma}

One proton study ${ }^{45}$ and 14 IMRT studies ${ }^{46-59}$ reported results for nasopharyngeal carcinomas. Estimates of 3-year LC were $92 \%$ for proton therapy and $95 \%$ for IMRT. Pooled 3-year OS estimates were $74 \%$ for proton therapy and $90 \%$ for IMRT. Both LC and OS were not statistically significantly different ( $P$-values 0.780 and 0.298 ).

The percentage of T4 patients in the proton study was approximately twice as high as in most IMRT studies (Appendix 3.4). Nevertheless, this covariate had no statistically significant influence on the outcome. The year of publication had a statistically significant (P-value 0.040) influence on the 3-year LC favoring older studies. The corrected estimates were similar to the uncorrected estimate for 3-year LC for both IMRT (both estimates were 95\%) and proton therapy (decreased from $92 \%$ to $90 \%$ ). As a result, differences in 3-year LC between IMRT and protons remained not statistically significant. The remaining covariates considering disease stage, the proportion of patients receiving chemotherapy or surgery were not statistically significant.

For proton therapy no late xerostomia or dysphagia data were reported, hence it was not possible to perform comparisons considering late treatment related toxicity.

\section{Oropharyngeal carcinoma}

One proton study ${ }^{60}$ and 10 IMRT studies ${ }^{61-71}$ reported results for oropharyngeal carcinomas. Estimates of 2- and 5-year LC were $96 \%$ and $88 \%$ for proton therapy and for IMRT pooled 2-year LC was $95 \%$. For DFS, 2- and 5-year rates were $81 \%$ and $65 \%$ for proton therapy, pooled 2 -year DFS for IMRT was $87 \%$. Both 2-year LC and DFS were not statistically significantly different (P-values 0.782 and 0.570 ).

No statistically significant covariates were observed.

For proton therapy no late xerostomia or dysphagia data were reported, hence it was not possible to perform comparisons considering late treatment related toxicity. 


\section{Nasopharynx}
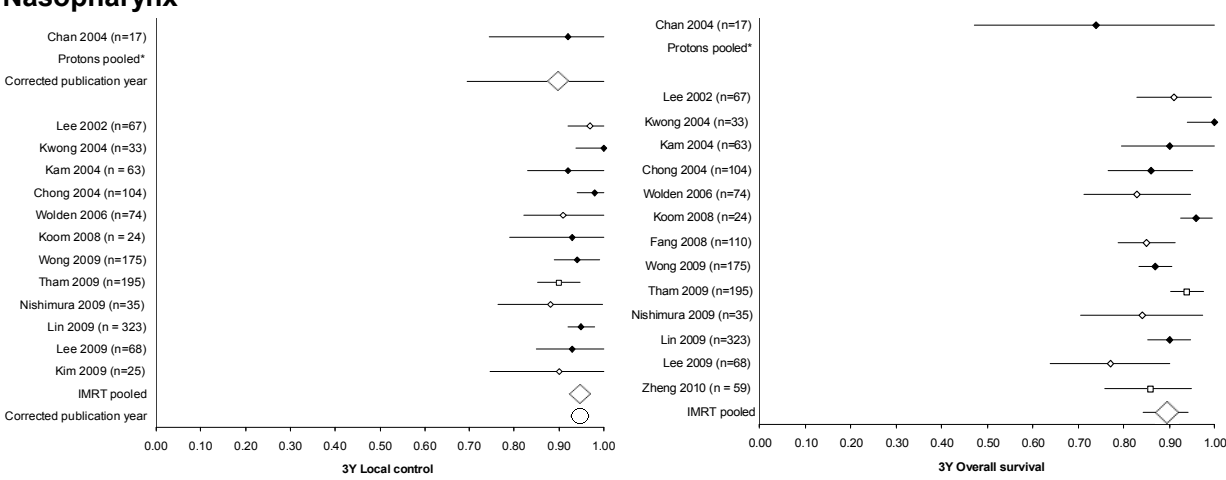

Oropharynx
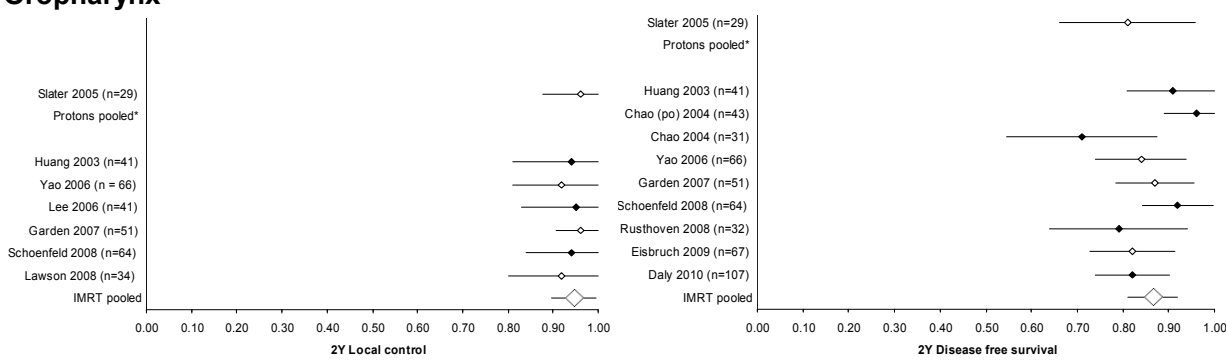

\section{Paranasal and sinonasal}
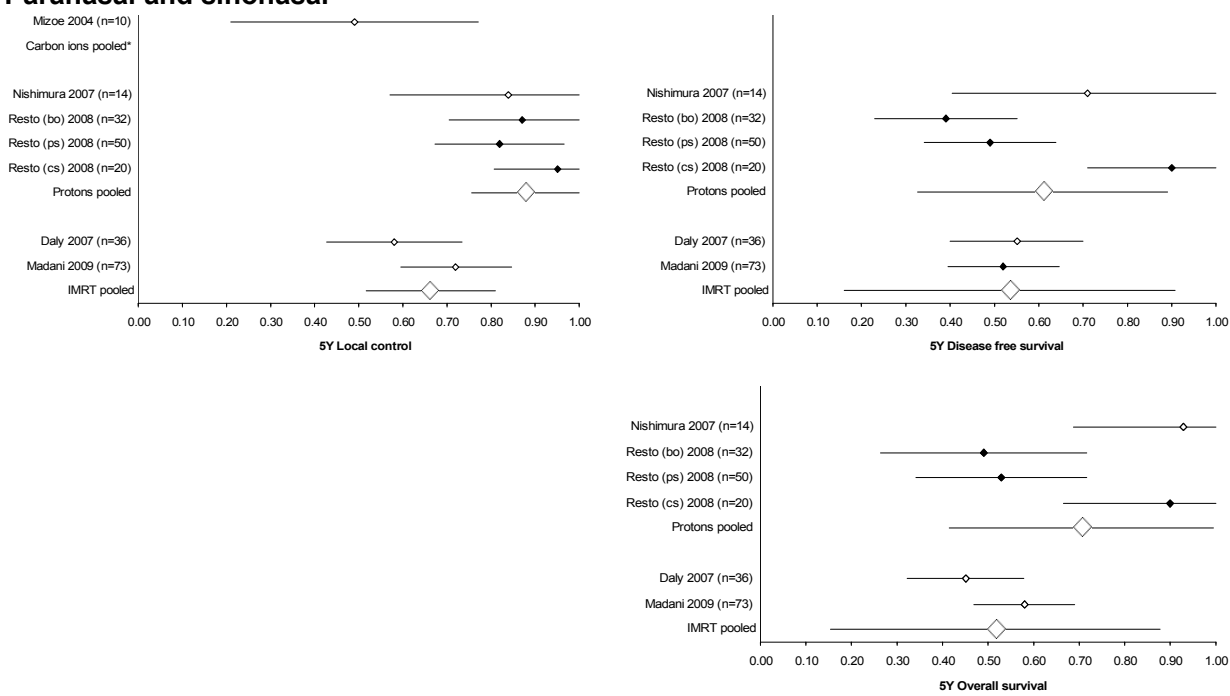

\section{Figure 3.1: Forest plots of random effect meta-regression}

Abbreviations: $p o=$ post-operative, bo $=$ biopsy only, $p s=$ partial surgery, $c s=$ complete surgery, $c t$ = chemotherapy

$\square=$ se retrieved from study, $\boldsymbol{\|}=$ se calculated (proportion method), $\diamond=$ se calculated (Peto method), - = se calculated (Peto method assuming maximum \% censoring reported in the particular subanalysis)

* Consisted of only 1 study and therefore no pooled estimate is reported

† Based on a study population consisting of $85 \%$ operated patients

$\ddagger$ Because of lacking data, it was not possible to correct for surgery for Carbon-ions 


\section{Mucosal malignant melanoma}

\section{Adenoid cystic Carcinoma}
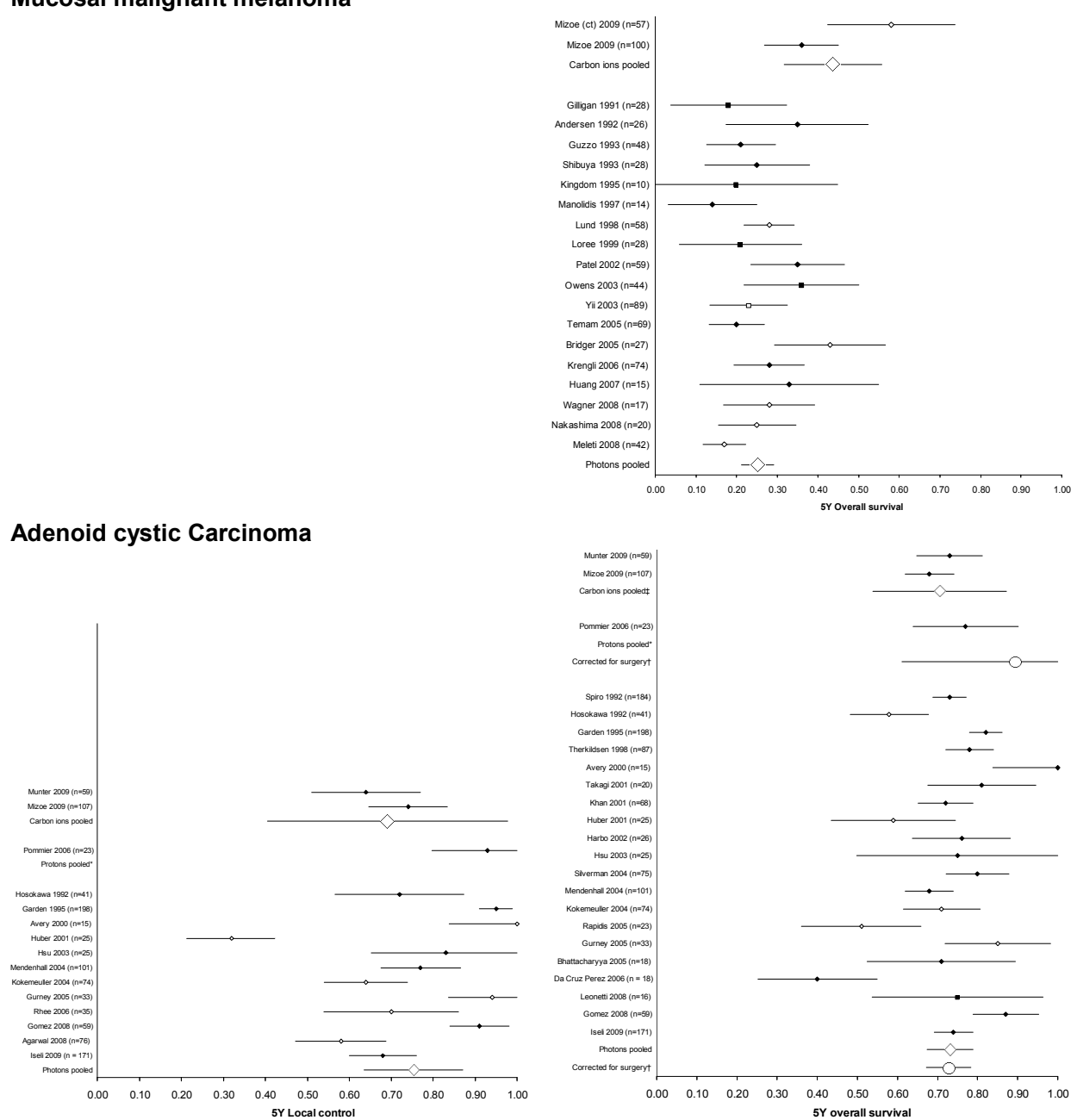

Figure 3.1 (continued): Forest plots of random effect meta-regression

Abbreviations: po $=$ post-operative, bo $=$ biopsy only, $p s=$ partial surgery, $c s=$ complete surgery, $c t$ $=$ chemotherapy

$\square=$ se retrieved from study, $\mathbf{\square}=$ se calculated (proportion method), $\diamond=$ se calculated (Peto method), - = se calculated (Peto method assuming maximum \% censoring reported in the particular subanalysis)

* Consisted of only 1 study and therefore no pooled estimate is reported

† Based on a study population consisting of $85 \%$ operated patients

$\ddagger$ Because of lacking data, it was not possible to correct for surgery for Carbon-ions 


\section{Paranasal and sinonasal carcinoma}

One carbon-ion study ${ }^{35}$ two proton studies ${ }^{72,73}$ and two IMRT studies ${ }^{74,75}$ reported results for paranasal or sinonasal carcinoma. The pooled estimates of 5 -year LC were $49 \%$ for carbon-ion therapy, $88 \%$ for proton therapy and $66 \%$ for IMRT. Differences in 5-year LC between proton therapy and IMRT were statistically significant (P-value 0.035 ). Five-year DFS was $61 \%$ for protons and $54 \%$ for IMRT, while 5 -year OS was $71 \%$ and $52 \%$ respectively. Both DFS and OS were not statistically significantly different (P-values 0.682 and 0.323 ).

It was not possible to include the percentage of patient with T4, N3 or Stage IV tumors as covariates in the meta-analyses since none of the included studies reported the number of Stage IV tumors and the number of T4 tumors or N3 tumors were only reported in the two photon studies (Appendix 3.4). None of the other covariates (including the percentage of operated patients) had a statistically significant influence on the outcome.

One proton study reported that no late visual toxicity of RTOG grade 3 or higher was observed. ${ }^{72}$ However, another study considering visual outcomes after combined photon/proton therapy reported LENT/CTC v2.0 visual toxicity of grade 1,2 and 3 of respectively $14 \%, 17 \%$ and $6 \% .{ }^{76}$ For IMRT, one study reported CTC v2.0 grade 3 visual toxicity in $9 \%$ of the patients, of which 4 patients $(6 \%)$ had pre-treatment visual impairment. ${ }^{75}$

\section{Mucosal malignant melanoma}

For the treatment of mucosal malignant melanomas, 5-year OS was reported in two carbon-ion studies ${ }^{34,77}$ and 18 studies administering conventional radiotherapy. ${ }^{78-95}$ Analyses showed a 5 -year OS of $44 \%$ for carbon-ion radiotherapy, this was statistically significantly higher than the pooled estimate of $25 \%$ for conventional photon radiotherapy ( $P$-value 0.007$)$.

The percentage of operated patients as well as the percentage of patients with disease stage III could not be incorporated as potentially confounding variables since none of the studies on carbon-ion therapy reported these data (Appendix 3.4). None of the other covariates were statistically significant.

For carbon-ion therapy one study observed late RTOG grade 2 skin toxicity in $3 \%$, another $3 \%$ had grade 2 mucosal toxicity and the remaining patients had grade $0-1$ toxicity. ${ }^{96}$ No photon studies reported skin or mucosal toxicity with accompanying grades. One study reported grade 3-4 visual toxicity in two $(8 \%)$ patients (one dry eye syndrome and one optic nerve toxicity resulting in decreased visual acuity) after photon radiotherapy. ${ }^{84}$ For carbon-ion therapy no visual toxicity data were reported, hence it was not possible to perform comparisons considering late treatment related visual toxicity. 
Table 3.1: Results of meta-analyses

\begin{tabular}{|c|c|c|c|c|c|}
\hline Treatment & Outcome $(95 \% \mathrm{Cl})$ & Comparison & Difference $(95 \% \mathrm{Cl})$ & $12(\%)$ & $\mathbf{P}^{*}$ \\
\hline \multicolumn{6}{|c|}{ Nasopharyngeal Carcinoma } \\
\hline & $3 Y$ LC & & & & \\
\hline IMRT & $\begin{array}{l}0.947 \\
(0.923 \text { to } 0.970)\end{array}$ & & & & \\
\hline \multirow[t]{2}{*}{ Protons $^{\dagger}$} & $\begin{array}{l}0.920 \\
(0.743 \text { to } 1.000)\end{array}$ & Protons - IMRT & $\begin{array}{l}-0.027 \\
(-0.232 \text { to } 0.178)\end{array}$ & 19.0 & 0.780 \\
\hline & $3 Y$ OS & & & & \\
\hline IMRT & $\begin{array}{l}0.897 \\
(0.862 \text { to } 0.933)\end{array}$ & & & & \\
\hline \multirow[t]{2}{*}{ Protons $^{\dagger}$} & $\begin{array}{l}0.740 \\
(0.471 \text { to } 1.000)\end{array}$ & Protons - IMRT & $\begin{array}{l}-0.157 \\
(-0.473 \text { to } 0.158)\end{array}$ & 67.5 & 0.298 \\
\hline & 3Y LC corrected for $t$ & ear of publication & & & \\
\hline IMRT & $\begin{array}{l}0.946 \\
(0.927 \text { to } 0.966)\end{array}$ & & & & \\
\hline Protons ${ }^{\dagger}$ & $\begin{array}{l}0.898 \\
(0.695 \text { to } 1.000)\end{array}$ & Protons - IMRT & $\begin{array}{l}-0.048 \\
(-0.252 \text { to } 0.156)\end{array}$ & 0.0 & 0.609 \\
\hline \multicolumn{6}{|c|}{ Oropharyngeal Carcinoma } \\
\hline & $2 Y L C$ & & & & \\
\hline IMRT & $\begin{array}{l}0.947 \\
(0.898 \text { to } 0.995)\end{array}$ & & & & \\
\hline \multirow[t]{2}{*}{ Protons $^{\dagger}$} & $\begin{array}{l}0.960 \\
(0.878 \text { to } 1.000)\end{array}$ & Protons - IMRT & $\begin{array}{l}0.013 \\
(-0.105 \text { to } 0.132)\end{array}$ & 0.0 & 0.782 \\
\hline & $2 Y$ DFS & & & & \\
\hline IMRT & $\begin{array}{l}0.865 \\
(0.812 \text { to } 0.918)\end{array}$ & & & & \\
\hline Protons ${ }^{\dagger}$ & $\begin{array}{l}0.810 \\
(0.662 \text { to } 0.958)\end{array}$ & Protons - IMRT & $\begin{array}{l}-0.055 \\
(-0.159 \text { to } 0.269)\end{array}$ & 49.9 & 0.570 \\
\hline \multicolumn{6}{|c|}{ Paranasal and Sinonasal Carcinoma } \\
\hline & $5 Y L C$ & & & & \\
\hline IMRT & $\begin{array}{l}0.662 \\
(0.516 \text { to } 0.809)\end{array}$ & Carbon-ions - IMRT & $\begin{array}{l}-0.172 \\
(-0.600 \text { to } 0.256)\end{array}$ & & 0.327 \\
\hline Protons & $\begin{array}{l}0.878 \\
(0.755 \text { to } 1.000)\end{array}$ & Protons - IMRT & $\begin{array}{l}0.216^{\ddagger} \\
(0.025 \text { to } 0.407)\end{array}$ & & 0.035 \\
\hline \multirow[t]{2}{*}{ Carbon-ions ${ }^{\dagger}$} & $\begin{array}{l}0.490 \\
(0.210 \text { to } 0.770)\end{array}$ & Protons - Carbon-ions & $\begin{array}{l}0.388 \\
(-0.033 \text { to } 0.809)\end{array}$ & 0.0 & 0.063 \\
\hline & $5 Y$ DFS & & & & \\
\hline IMRT & $\begin{array}{l}0.535 \\
(0.162 \text { to } 0.907)\end{array}$ & & & & \\
\hline \multirow[t]{2}{*}{ Protons } & $\begin{array}{l}0.609 \\
(0.326 \text { to } 0.891)\end{array}$ & Protons - IMRT & $\begin{array}{l}0.074 \\
(-0.393 \text { to } 0.542)\end{array}$ & 78.2 & 0.682 \\
\hline & $5 Y$ OS & & & & \\
\hline IMRT & $\begin{array}{l}0.516 \\
(0.154 \text { to } 0.878)\end{array}$ & & & & \\
\hline Protons & $\begin{array}{l}0.705 \\
(0.414 \text { to } 0.995)\end{array}$ & Protons - IMRT & $\begin{array}{l}0.188 \\
(-0.276 \text { to } 0.653)\end{array}$ & 73.2 & 0.323 \\
\hline
\end{tabular}

* P-value for the difference between the treatments

$\dagger$ Consisted of only one study and the study estimated instead of the pooled estimate is therefore reported

$\ddagger$ Statistically significant difference 
Table 3.1 (continued): Results of meta-analyses

\begin{tabular}{|c|c|c|c|c|c|}
\hline Treatment & Outcome $(95 \% \mathrm{Cl})$ & Comparison & Difference $(95 \% \mathrm{Cl})$ & $1^{2}(\%)$ & $\mathbf{P}^{*}$ \\
\hline \multicolumn{6}{|c|}{ Mucosal Malignant Melanoma } \\
\hline & $5 Y$ OS & & & & \\
\hline Photons & $\begin{array}{l}0.252 \\
(0.212 \text { to } 0.291)\end{array}$ & & & & \\
\hline Carbon-ions & $\begin{array}{l}0.437 \\
(0.316 \text { to } 0.558)\end{array}$ & Carbon-ions - Photons & $\begin{array}{l}0.185^{\ddagger} \\
(0.058 \text { to } 0.313)\end{array}$ & 51.7 & 0.007 \\
\hline \multicolumn{6}{|c|}{ Adenoid Cystic Carcinoma } \\
\hline & $5 Y L C$ & & & & \\
\hline Photons & $\begin{array}{l}0.753 \\
(0.635 \text { to } 0.870)\end{array}$ & Carbon-ions - Photons & $\begin{array}{l}0.061 \\
(-0.249 \text { to } 0.371)\end{array}$ & & 0.675 \\
\hline Protons $^{\dagger}$ & $\begin{array}{l}0.930 \\
(0.797 \text { to } 1.000)\end{array}$ & Protons - Photons & $\begin{array}{l}0.177 \\
(-0.252 \text { to } 0.607)\end{array}$ & & 0.386 \\
\hline \multirow[t]{2}{*}{ Carbon-ions } & $\begin{array}{l}0.691 \\
(0.405 \text { to } 0.978)\end{array}$ & Protons - Carbon-ions & $\begin{array}{l}0.239 \\
(-0.264 \text { to } 0.741)\end{array}$ & 93.7 & 0.322 \\
\hline & $5 Y$ OS & & & & \\
\hline Photons & $\begin{array}{l}0.731 \\
(0.674 \text { to } 0.789)\end{array}$ & Carbon-ions - Photons & $\begin{array}{l}-0.027 \\
(-0.203 \text { to } 0.149)\end{array}$ & & 0.752 \\
\hline Protons $^{\dagger}$ & $\begin{array}{l}0.770 \\
(0.639 \text { to } 0.901)\end{array}$ & Protons - Photons & $\begin{array}{l}0.039 \\
(-0.233 \text { to } 0.310)\end{array}$ & & 0.769 \\
\hline \multirow[t]{2}{*}{ Carbon-ions } & $\begin{array}{l}0.704 \\
(0.538 \text { to } 0.871)\end{array}$ & Protons - Carbon-ions & $\begin{array}{l}0.066 \\
(-0.248 \text { to } 0.379)\end{array}$ & 77.8 & 0.666 \\
\hline & \multicolumn{5}{|c|}{ 5Y OS corrected for the percentage of operated patient } \\
\hline Photons & $\begin{array}{l}0.728 \\
(0.672 \text { to } 0.784)\end{array}$ & & & & \\
\hline Protons & $\begin{array}{l}0.893 \\
(0.611 \text { to } 1.000)\end{array}$ & Protons - Photons & $\begin{array}{l}0.165 \\
(-0.124 \text { to } 0.455)\end{array}$ & 73.6 & 0.245 \\
\hline
\end{tabular}

* P-value for the difference between the treatments

† Consisted of only one study and the study estimated instead of the pooled estimate is therefore reported

$\ddagger$ Statistically significant difference

\section{Adenoid cystic carcinoma}

For adenoid cystic carcinomas (ACC), one non-randomized comparative study which compared carbon-ion therapy $(n=29)$ with photon radiation $(n=34)$ showed no statistically significant differences. ${ }^{97}$ Further, two carbon-ion studies (including updated data of the comparative study), ${ }^{34,98}$ one proton study ${ }^{99}$ and 22 conventional photon studies ${ }^{100-121}$ reported results for adenoid cystic carcinomas.

Meta-analyses resulted in a pooled 5 -year LC of $69 \%$ for carbon-ion therapy, $93 \%$ for proton therapy and $75 \%$ for conventional photon therapy. Pooled 5 year estimates for OS were $70 \%$ for carbon-ion therapy, $77 \%$ for proton therapy and $73 \%$ for photon therapy. No statistically significant differences were observed between carbon-ion therapy, proton therapy and conventional photon therapy for 5-year LC and OS (see table 3.1 for P-values). 
The percentage of operated patients had a statistically significant influence on OS (P-value 0.043 ) favoring studies with higher proportions of operated patients. Therefore 5-year OS estimates were corrected for this covariate. Due to lacking data, this was not possible for carbon-ion therapy (Appendix 3.4). The corrected estimate was similar to the uncorrected estimate for 5-year OS for photon therapy (both 73\%), but for proton therapy the corrected estimate increased from $77 \%$ to $89 \%$. After correcting 5 -year OS, the differences between IMRT and protons remained not statistically significant. The covariates considering disease stage and T4 tumors were not reported in the particle studies and were therefore not included in the analyses. The remaining covariates had no statistically significant influence on the outcome.

For carbon-ion radiotherapy, late CTC 13.0 grade 1-2 toxicity was observed in $63 \%$ of the patients and no toxicity of grade 3 or higher was reported. ${ }^{98}$ Reported RTOG late grade 1-2 toxicity in photon studies was $64 \%$ and $84 \%{ }^{105,118}$ and ranged between $4 \%$ and $11 \%$ for grade 3 and higher toxicity.

\section{Comparative in-silico studies}

Eight comparative in-silico studies were included, one ${ }^{122}$ comparing carbon-ion therapy and photon therapy and seven ${ }^{123-129}$ comparing proton therapy and photon therapy.

One in-silico study suggested that for paranasal sinus tumors, carbon-ion therapy has the ability to statistically significantly decrease the dose to the contralateral optic nerve. ${ }^{122}$ However, due to unknown differences in relative biological effectiveness for carbon-ion therapy, it is yet unclear how these differences in dose will translate into clinically relevant outcomes. Also, for proton therapy one in-silico study reported a lower dose to the optic nerves compared with IMRT (Table 3.2). ${ }^{125}$ No information was provided on to what extent this reduced dose would reduce the probability of visual impairment.

For nasopharyngeal, oropharyngeal, hypopharyngeal and squamous cell carcinomas, six comparative in-silico studies consistently showed a lower dose to the parotid glands for proton therapy as opposed to IMRT (Table 3.2). The decrease in mean dose to the parotid glands between IMRT and proton therapy ranged from $2 \mathrm{GyE}$ to $10 \mathrm{GyE}$. Based on normal tissue complication probability (NTCP) models, three studies ${ }^{124,128,129}$ predicted probability of complications of the parotid glands (defined as a decrease of the salivary flow to $25 \%$ of the pretreatment salivary flow). The absolute decrease (percentage points) of the complication probability of the parotid glands for proton therapy compared with IMRT ranged from $6 \%$ to $28 \%$ (Table 3.2 ).

None of the included in-silico studies reported dose to the pharyngeal constrictors. 


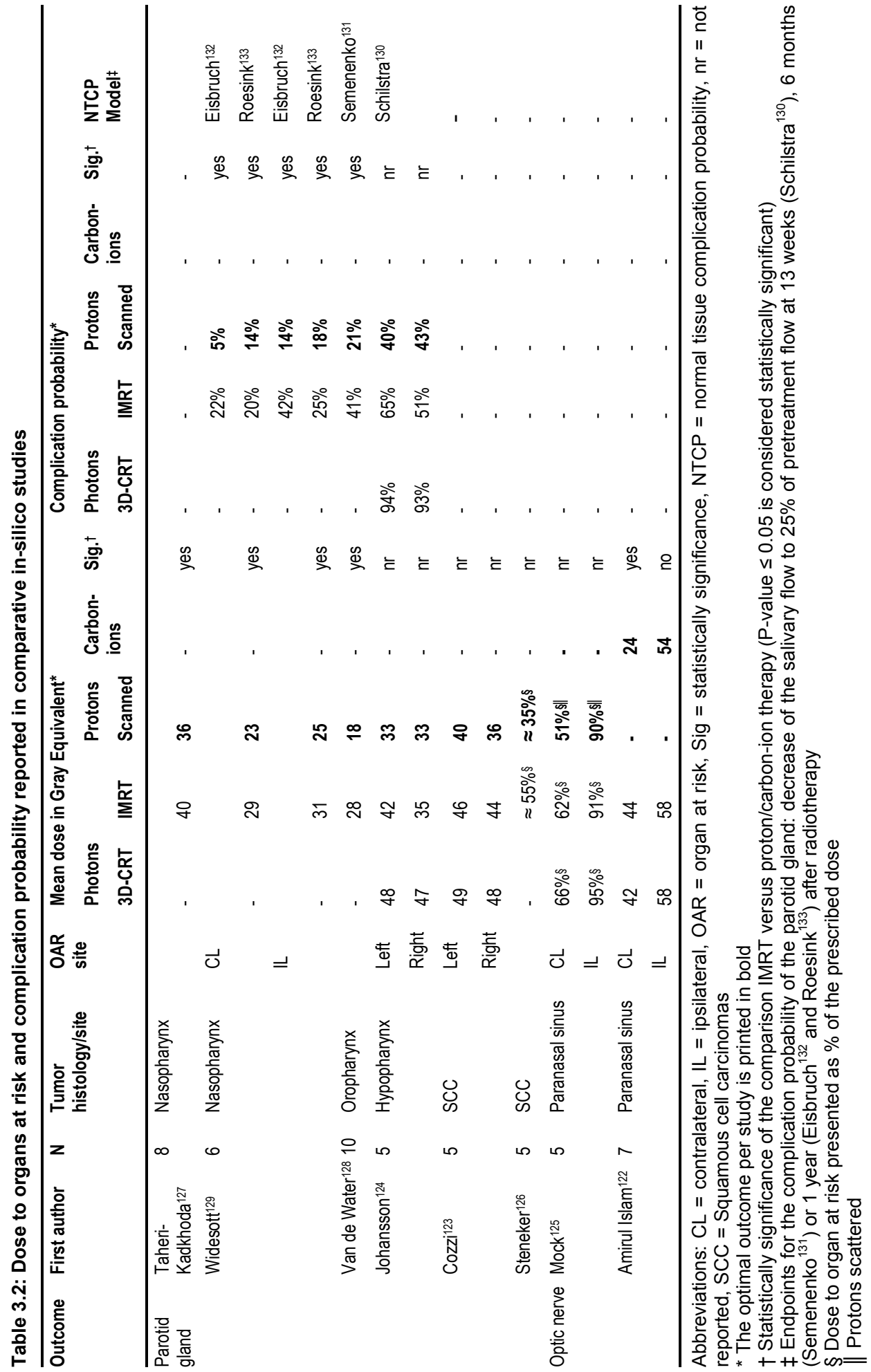


The reduced dose to organs at risk for carbon-ion and proton therapy was accomplished with a similar conformity index, dose homogeneity and coverage of the target volume for particle therapy. Hence, the decreased dose to the organs at risk will not result in a reduced tumor control probability.

\section{Discussion}

To the best of our knowledge, this review and meta-analysis was the first that reviewed all evidence (clinical and in-silico studies) and pooled the effectiveness of photon, carbon-ion and proton radiotherapy in HNC. Based on currently available evidence, we found that carbon-ion radiotherapy resulted in a statistically significantly increased 5-year OS compared to conventional photon therapy for mucosal malignant melanomas. Melanomas are considered as relatively radio-resistant tumors. ${ }^{134,135}$ Therefore, our results might indicate that carbonion therapy is advantageous in the treatment of relatively radio-resistant tumors in the head and neck region, which profit from the increased relative biological effectiveness. Because of lacking data, no comparisons were performed considering toxicity in mucosal malignant melanomas. Toxicity data for adenoid cystic toxicity was based on different classification systems (RTOG and CTC), which complicated the comparison. However, the incidence of toxicity for carbon-ion therapy in adenoid cystic carcinomas seemed lower than reported in conventional photon studies.

Outcomes for proton therapy were generally comparable with the best available photon therapy in nasopharyngeal, oropharyngeal and adenoid cystic carcinomas. For paranasal and sinonasal cancer, DFS and OS were comparable for photon and proton therapy. Nevertheless, LC was statistically significantly increased for proton therapy. Besides a higher total treatment dose, this increase may also be influenced by the proportion of patients that received surgery (Appendix 3.4). ${ }^{73}$ For the remainder, the similar outcomes can be explained since the radiobiological differences between protons and photons are small and the total treatment doses among studies were generally comparable. Considering treatment toxicity in oropharyngeal cancer and nasopharyngeal cancer, no appropriate data concerning xerostomia or dysphagia were available to perform comparisons based on clinical data. Although based on different classification systems, in paranasal and sinonasal cancer the incidence of grade 3 visual toxicity seemed lower for proton therapy as compared to photon therapy.

In paranasal carcinomas, one comparative in-silico studies showed a reduced dose to the optic nerves for carbon-ion therapy compared to photon therapy. ${ }^{122}$ Nonetheless, it is not yet clear how this decrease in dose translates into clinical outcomes. For proton therapy compared to photon therapy, we found a de- 
creased dose to the optical nerves ${ }^{125}$ and to the parotid glands. ${ }^{123-129}$ Based on NTCP models, this latter dose reduction resulted in a lower complication probability for the parotid glands. ${ }^{124,128,129}$ However, the reported complication probabilities varied largely among the in-silico studies, which is partly due to differences in the applied NTCP models. The decreased dose to the organs at risk was accomplished without compromising on the conformity index, dose homogeneity and coverage of the target volume. ${ }^{123-129}$ This indicates that the main advantage of proton therapy in $\mathrm{HNC}$ may currently be the ability to spare organs at risk without decreasing tumor control rates. However, clinical evidence to support this is not yet available.

To explore potential confounding factors and subsequently correct for heterogeneity between studies we included covariates in our analyses. In two analyses, statistically significantly influencing covariates were observed. Firstly, the year of publication was statistically significant in the analyses concerning 3-year LC in nasopharyngeal carcinomas, which might reflect changes over time such as the inclusion of more patients with nodal extension in more recent studies (Appendix 3.4). However, this covariate did not substantially affect the estimated 3-year LC. Secondly, in the analyses considering adenoid cystic carcinomas, the proportion of operated patients had a statistically significant influence on 5year OS. The estimate for conventional photon therapy remained unchanged whereas the 5-year OS for proton therapy increased substantially (Figure 3.1 and Table 3.1). The remaining analyses did not reveal other covariates which statistically significantly influenced the outcomes.

Radiotherapy was often combined with chemotherapy and/or surgery in the included studies. Since our analyses were restricted to group-level data and study outcomes were not stratified for chemotherapy and surgery, we were not able to clarify the individual impact of chemotherapy or the positioning of surgery. It would be an interesting topic for future research to examine whether and when proton or carbon-ion radiotherapy could best be combined with chemotherapy and/or surgery.

Further, the potential gain of particle therapy in terms of tumor control may rely on higher doses to the tumor while remaining an equal dose to the organs at risk. Correcting for differences in treatment dose would therefore remove the potential gain of particle therapy. For that reason, our analyses were not corrected for differences in radiotherapy dosage schemes.

The present study has some limitations. Most importantly, the evidence on the effectiveness of particle therapy consists of a limited number of (mainly small) observational studies. Also, influencing variables were not always reported adequately. As a consequence correcting for confounding variables was only possible to a limited extent. These data issues may bias the comparisons as a result of differences in setting or treatment properties. This is considered a main drawback of meta-analysis of observational studies. ${ }^{33,136}$ However, in the absence of randomized trials, meta-analysis of observational studies is one of the 
few methods to synthesize effectiveness and present uncertainty ${ }^{33}$ and follows the recommendation that all relevant studies should be taken into account. ${ }^{137}$ Another data concern was the scarcely reported toxicity data among studies, and as a result it was not possible to adequately compare late treatment toxicity based on clinical data.

\section{Implications for practice}

It has been subject of debate whether the high initial and treatment costs of particle therapy are justified given the currently available evidence surrounding its effectiveness. ${ }^{21}$ Based on the available evidence, particle therapy seems as least as effective as photon therapy in tumor control, and may have advantages in sparing organs at risk. However, with regard to the paucity of available proton and carbon-ion studies and the potential bias of comparisons between (small) observational studies, the superiority of particle therapy in terms of tumor control and survival as well as the question whether the benefits outweigh the costs remains uncertain.

\section{Implications for research}

Particles are used in the treatment of cancer since $1954 .{ }^{138}$ However, the number of published studies which administer particle therapy in HNC is limited and much of the currently available data have been gathered in less than optimal physic-based settings. Therefore, it is difficult to establish the potential effectiveness of particle therapy in more optimal clinical settings. Studies evaluating the effectiveness of particle therapy in HNC stratified by location and/or histology are therefore needed.

Curative radiation therapy for HNC is often accompanied with a trade-off between radiation toxicity and survival. The length of survival alone is therefore insufficient to determine the success of a treatment, and the quality of survival needs to be considered simultaneously. ${ }^{139}$ However, complete (late) treatment toxicity data is infrequently and inconsistently reported. Hence, there is a need for studies to systematically examine both minor and severe radiation toxicity per type and grade. This is especially important if decreasing treatment toxicity is regarded as the potential benefit of particle therapy in HNC.

As recommended previously, ${ }^{18}$ to facilitate comparisons on both survival and treatment toxicity between particle therapy and photon radiation; the construction of an international particle therapy register, which contains anonymous data from patients who have been treated with particle therapy, would be a straightforward and effective solution. Such registries become particularly evident if randomized trials comparing photon therapy and particle therapy are not to be expected. $^{140}$ 
For now, in the absence of comprehensive toxicity data, comparative in-silico studies combined with dose-response models offer a complementary way of estimating the potential added value of particle therapy in $\mathrm{HNC}$ in relation to its costs. $^{15,22}$

\section{Conclusions}

Currently available clinical evidence for HNC indicates that survival for carbonion radiotherapy in mucosal malignant melanomas is statistically significantly increased as opposed to photon radiation. This suggests that carbon-ion radiotherapy, with its increased biological efficacy, might be advantageous in the treatment of relatively radio-resistant tumors. Besides local control in paranasal and sinonasal cancer, survival and tumor control outcomes for proton therapy were to a large extent similar to the best available photon radiation. Based on limited data, this review indicates that a clinically relevant benefit of proton therapy probably lies in reducing treatment toxicity in HNC. This finding is in accordance with available comparative in-silico studies.

Since the overall quantity and quality of data regarding carbon-ion and proton therapy is poor and considering the risk of bias in comparisons between (small) observational studies, the reported results do not allow for definite conclusions. Therefore, we recommend the construction of an international particle therapy register to facilitate definitive comparisons on both survival and treatment toxicity between particle and photon radiotherapy.

\section{Acknowledgement}

Jun-etsu Mizoe, Luca Cozzi and Antonella Fogliata are gratefully acknowledged for providing additional data. 


\section{References}

1. Ferlay $\mathrm{J}$, Autier $\mathrm{P}$, Boniol $\mathrm{M}$, et al: Estimates of the cancer incidence and mortality in Europe in 2006. Ann Oncol 18:581-92, 2007

2. Baujat $\mathrm{B}$, Audry H, Bourhis $\mathrm{J}$, et al: Chemotherapy in locally advanced nasopharyngeal carcinoma: an individual patient data meta-analysis of eight randomized trials and 1753 patients. Int J Radiat Oncol Biol Phys 64:47-56, 2006

3. Bourhis J, Overgaard J, Audry H, et al: Hyperfractionated or accelerated radiotherapy in head and neck cancer: a meta-analysis. Lancet 368:843-54, 2006

4. Pignon JP, le Maitre A, Maillard E, et al: Meta-analysis of chemotherapy in head and neck cancer (MACH-NC): an update on 93 randomised trials and 17,346 patients. Radiother Oncol 92:4-14, 2009

5. Duke RL, Campbell BH, Indresano AT, et al: Dental status and quality of life in long-term head and neck cancer survivors. Laryngoscope 115:678-83, 2005

6. Hammerlid E, Silander E, Hornestam L, et al: Health-related quality of life three years after diagnosis of head and neck cancer--a longitudinal study. Head Neck 23:113-25, 2001

7. Jensen $A B$, Hansen $O$, Jorgensen $K$, et al: Influence of late side-effects upon daily life after radiotherapy for laryngeal and pharyngeal cancer. Acta Oncol 33:487-91, 1994

8. Langendijk JA, Doornaert P, Verdonck-de Leeuw IM, et al: Impact of late treatment-related toxicity on quality of life among patients with head and neck cancer treated with radiotherapy. $J$ Clin Oncol 26:3770-6, 2008

9. List MA, Bilir SP: Functional outcomes in head and neck cancer. Semin Radiat Oncol 14:17889, 2004

10. Durante M, Loeffler JS: Charged particles in radiation oncology. Nat Rev Clin Oncol 7:37-43, 2010

11. Glimelius B, Ask A, Bjelkengren $G$, et al: Number of patients potentially eligible for proton therapy. Acta Oncol 44:836-49, 2005

12. Palm A, Johansson KA: A review of the impact of photon and proton external beam radiotherapy treatment modalities on the dose distribution in field and out-of-field; implications for the long-term morbidity of cancer survivors. Acta Oncol 46:462-73, 2007

13. Ask A, Bjork-Eriksson $T$, Zackrisson $B$, et al: The potential of proton beam radiation therapy in head and neck cancer. Acta Oncol 44:876-80, 2005

14. Brada M, Pijls-Johannesma M, De Ruysscher D: Proton therapy in clinical practice: current clinical evidence. J Clin Oncol 25:965-70, 2007

15. Brada M, Pijls-Johannesma M, De Ruysscher D: Current Clinical Evidence for Proton therapy. The Cancer Journal 15:319-324, 2009

16. Chan AW, Liebsch NJ: Proton radiation therapy for head and neck cancer. J Surg Oncol 97:697-700, 2008

17. Jereczek-Fossa BA, Krengli M, Orecchia R: Particle beam radiotherapy for head and neck tumors: radiobiological basis and clinical experience. Head Neck 28:750-60, 2006

18. Lodge M, Pijls-Johannesma M, Stirk L, et al: A systematic literature review of the clinical and cost-effectiveness of hadron therapy in cancer. Radiother Oncol 83:110-22, 2007

19. Schulz-Ertner D, Jakel O, Schlegel W: Radiation therapy with charged particles. Semin Radiat Oncol 16:249-59, 2006

20. Schulz-Ertner D, Tsujii H: Particle radiation therapy using proton and heavier ion beams. J Clin Oncol 25:953-64, 2007

21. Pijls-Johannesma $M$, Pommier $P$, Lievens $Y$ : Cost-effectiveness of particle therapy: current evidence and future needs. Radiother Oncol 89:127-34, 2008

22. Glimelius $B$, Isacsson $U$, Blomquist $E$, et al: Potential gains using high-energy protons for therapy of malignant tumours. Acta Oncol 38:137-45, 1999

23. Richardson WS, Wilson MC, Nishikawa J, et al: The well-built clinical question: a key to evidence-based decisions. ACP J Club 123:A12-3, 1995 
24. Feuvret L, Noel G, Mazeron JJ, et al: Conformity index: a review. Int J Radiat Oncol Biol Phys 64:333-42, 2006

25. Mayo C, Martel MK, Marks LB, et al: Radiation dose-volume effects of optic nerves and chiasm. Int J Radiat Oncol Biol Phys 76:S28-35, 2010

26. Deasy JO, Moiseenko V, Marks L, et al: Radiotherapy dose-volume effects on salivary gland function. Int J Radiat Oncol Biol Phys 76:S58-63, 2010

27. Caudell JJ, Burnett OL, Schaner PE, et al: Comparison of methods to reduce dose to swallowing-related structures in head and neck cancer. Int J Radiat Oncol Biol Phys 77:462-7, 2010

28. Rancati T, Schwarz M, Allen AM, et al: Radiation dose-volume effects in the larynx and pharynx. Int J Radiat Oncol Biol Phys 76:S64-9, 2010

29. Moore DS, McCabe GP, Craig BA: Introduction to the practice of statistics (ed 6th), W.H. Freeman Company, 2009

30. Cantor AB: Projecting the standard error of the Kaplan-Meier estimator. Stat Med 20:2091-7, 2001

31. Peto R, Pike MC, Armitage $P$, et al: Design and analysis of randomized clinical trials requiring prolonged observation of each patient: II analysis and examples. Britisch Journal of Cancer 35:1-39, 1973

32. Thompson SG, Higgins JP: How should meta-regression analyses be undertaken and interpreted? Stat Med 21:1559-73, 2002

33. Stroup DF, Berlin JA, Morton SC, et al: Meta-analysis of observational studies in epidemiology: a proposal for reporting. Meta-analysis Of Observational Studies in Epidemiology (MOOSE) group. Jama 283:2008-12, 2000

34. Mizoe JE, Hasegawa A, Bessho $\mathrm{H}$, et al: Head and Neck Tumors, Proceedings of NIRSETOILE: Joint symposium 2009 on carbon ion radiotherapy. Lyon, 2009, pp 32-35

35. Mizoe JE, Tsujii H, Kamada T, et al: Dose escalation study of carbon ion radiotherapy for locally advanced head-and-neck cancer. Int J Radiat Oncol Biol Phys 60:358-64, 2004

36. Tokuuye $\mathrm{K}$, Akine $\mathrm{Y}$, Kagei $\mathrm{K}$, et al: Proton therapy for head and neck malignancies at Tsukuba. Strahlenther Onkol 180:96-101, 2004

37. Lauve A, Morris M, Schmidt-Ullrich R, et al: Simultaneous integrated boost intensity-modulated radiotherapy for locally advanced head-and-neck squamous cell carcinomas: II--clinical results. Int J Radiat Oncol Biol Phys 60:374-87, 2004

38. Milano MT, Vokes EE, Kao J, et al: Intensity-modulated radiation therapy in advanced head and neck patients treated with intensive chemoradiotherapy: preliminary experience and future directions. Int J Oncol 28:1141-51, 2006

39. Monroe AT, Young JA, Huff JD, et al: Accelerated fractionation head and neck intensitymodulated radiation therapy and concurrent chemotherapy in the community setting: safety and efficacy considerations. Head Neck 31:1144-51, 2009

40. Nangia S, Chufal KS, Tyagi A, et al: Selective Nodal Irradiation for Head and Neck Cancer Using Intensity-Modulated Radiotherapy: Application of RTOG Consensus Guidelines in Routine Clinical Practice. Int J Radiat Oncol Biol Phys, 2009

41. Rades D, Fehlauer F, Wroblesky J, et al: Prognostic factors in head-and-neck cancer patients treated with surgery followed by intensity-modulated radiotherapy (IMRT), 3D-conformal radiotherapy, or conventional radiotherapy. Oral Oncol 43:535-43, 2007

42. Saba NF, Edelman S, Tighiouart M, et al: Concurrent chemotherapy with intensity-modulated radiation therapy for locally advanced squamous cell carcinoma of the larynx and oropharynx: A retrospective single-institution analysis. Head Neck 31:1447-55, 2009

43. Seung S, Bae J, Solhjem M, et al: Intensity-modulated radiotherapy for head-and-neck cancer in the community setting. Int J Radiat Oncol Biol Phys 72:1075-81, 2008

44. Studer G, Furrer K, Davis BJ, et al: Postoperative IMRT in head and neck cancer. Radiat Oncol $1: 40,2006$

45. Chan AW, Liebsch LJ, Deschler DG, et al: Proton radiotherapy for T4 nasopharyngeal carcinoma. J Clin Oncol (Meeting Abstracts) 22:5574-, 2004 
46. Chong Z: Improved Local Control with Intensity Modulated Radiation Therapy in Patients with Nasopharyngeal Carcinoma. Int J Radiat Oncol Biol Phys 60:S317, 2004

47. Fang FM, Chien CY, Tsai WL, et al: Quality of life and survival outcome for patients with nasopharyngeal carcinoma receiving three-dimensional conformal radiotherapy vs. intensitymodulated radiotherapy-a longitudinal study. Int J Radiat Oncol Biol Phys 72:356-64, 2008

48. Kam MK, Teo PM, Chau RM, et al: Treatment of nasopharyngeal carcinoma with intensitymodulated radiotherapy: the Hong Kong experience. Int J Radiat Oncol Biol Phys 60:1440-50, 2004

49. Kim K, Wu HG, Kim HJ, et al: Intensity-modulated radiation therapy with simultaneous integrated boost technique following neoadjuvant chemotherapy for locoregionally advanced nasopharyngeal carcinoma. Head Neck 31:1121-8, 2009

50. Koom WS, Kim TH, Shin KH, et al: SMART (simultaneous modulated accelerated radiotherapy) for locally advanced nasopharyngeal carcinomas. Head Neck 30:159-69, 2008

51. Kwong DL, Pow EH, Sham JS, et al: Intensity-modulated radiotherapy for early-stage nasopharyngeal carcinoma: a prospective study on disease control and preservation of salivary function. Cancer 101:1584-93, 2004

52. Lee N, Harris J, Garden AS, et al: Intensity-modulated radiation therapy with or without chemotherapy for nasopharyngeal carcinoma: radiation therapy oncology group phase II trial 0225. J Clin Oncol 27:3684-90, 2009

53. Lee N, Xia P, Quivey JM, et al: Intensity-modulated radiotherapy in the treatment of nasopharyngeal carcinoma: an update of the UCSF experience. Int J Radiat Oncol Biol Phys 53:12-22, 2002

54. Lin S, Pan J, Han L, et al: Nasopharyngeal Carcinoma Treated with Reduced-Volume IntensityModulated Radiation Therapy: Report on the 3-Year Outcome of a Prospective Series. Int $\mathrm{J}$ Radiat Oncol Biol Phys 75:1071-8, 2009

55. Nishimura Y, Shibata T, Nakamatsu K, et al: A Two-step Intensity-modulated Radiation Therapy Method for Nasopharyngeal Cancer: The Kinki University Experience. Jpn J Clin Oncol 40:130-8., 2009

56. Tham IW, Hee SW, Yeo RM, et al: Treatment of Nasopharyngeal Carcinoma Using IntensityModulated Radiotherapy-The National Cancer Centre Singapore Experience. Int J Radiat Oncol Biol Phys 75:1481-6, 2009

57. Wolden SL, Chen WC, Pfister DG, et al: Intensity-modulated radiation therapy (IMRT) for nasopharynx cancer: update of the Memorial Sloan-Kettering experience. Int J Radiat Oncol Biol Phys 64:57-62, 2006

58. Wong FC, Ng AW, Lee VH, et al: Whole-Field Simultaneous Integrated-Boost IntensityModulated Radiotherapy for Patients with Nasopharyngeal Carcinoma. Int J Radiat Oncol Biol Phys 76:138-45, 2009

59. Zheng J, Wang G, Yang GY, et al: Induction Chemotherapy with Nedaplatin with 5-FU Followed by Intensity-modulated Radiotherapy Concurrent with Chemotherapy for Locoregionally Advanced Nasopharyngeal Carcinoma. Jpn J Clin Oncol:[Epub ahead of print], 2010

60. Slater JD, Yonemoto LT, Mantik DW, et al: Proton radiation for treatment of cancer of the oropharynx: early experience at Loma Linda University Medical Center using a concomitant boost technique. Int J Radiat Oncol Biol Phys 62:494-500, 2005

61. Auperin A, Le Pechoux C, Rolland E, et al: Meta-analysis of concomitant versus sequential radiochemotherapy in locally advanced non-small-cell lung cancer. J Clin Oncol 28:2181-90, 2010

62. Daly ME, Le QT, Maxim PG, et al: Intensity-Modulated Radiotherapy in the Treatment of Oropharyngeal Cancer: Clinical Outcomes and Patterns of Failure. Int J Radiat Oncol Biol Phys 76:1339-46, 2010

63. Eisbruch A, Harris J, Garden AS, et al: Multi-Institutional Trial of Accelerated Hypofractionated Intensity-Modulated Radiation Therapy for Early-Stage Oropharyngeal Cancer (RTOG 00-22). Int J Radiat Oncol Biol Phys, 2009 
64. Garden AS, Morrison WH, Wong PF, et al: Disease-control rates following intensity-modulated radiation therapy for small primary oropharyngeal carcinoma. Int J Radiat Oncol Biol Phys 67:438-44, 2007

65. Huang K, Lee N, Xia P, et al: Intensity-modulated radiotherapy in the treatment of oropharyngeal carcinoma: a single institutional experience. Int J Radiat Oncol Biol Phys 57:S302, 2003

66. Lawson JD, Otto K, Chen A, et al: Concurrent platinum-based chemotherapy and simultaneous modulated accelerated radiation therapy for locally advanced squamous cell carcinoma of the tongue base. Head Neck 30:327-35, 2008

67. Lee NY, de Arruda FF, Puri DR, et al: A comparison of intensity-modulated radiation therapy and concomitant boost radiotherapy in the setting of concurrent chemotherapy for locally advanced oropharyngeal carcinoma. Int J Radiat Oncol Biol Phys 66:966-74, 2006

68. Rusthoven KE, Raben D, Ballonoff $A$, et al: Effect of radiation techniques in treatment of oropharynx cancer. Laryngoscope 118:635-9, 2008

69. Schoenfeld GO, Amdur RJ, Morris CG, et al: Patterns of failure and toxicity after intensitymodulated radiotherapy for head and neck cancer. Int J Radiat Oncol Biol Phys 71:377-85, 2008

70. Yao M, Nguyen T, Buatti JM, et al: Changing failure patterns in oropharyngeal squamous cell carcinoma treated with intensity modulated radiotherapy and implications for future research. Am J Clin Oncol 29:606-12, 2006

71. Chao KS, Ozyigit G, Blanco Al, et al: Intensity-modulated radiation therapy for oropharyngeal carcinoma: impact of tumor volume. Int J Radiat Oncol Biol Phys 59:43-50, 2004

72. Nishimura $\mathrm{H}$, Ogino $\mathrm{T}$, Kawashima $\mathrm{M}$, et al: Proton-beam therapy for olfactory neuroblastoma. Int J Radiat Oncol Biol Phys 68:758-62, 2007

73. Resto VA, Chan AW, Deschler DG, et al: Extent of surgery in the management of locally advanced sinonasal malignancies. Head Neck 30:222-9, 2008

74. Daly ME, Chen AM, Bucci MK, et al: Intensity-modulated radiation therapy for malignancies of the nasal cavity and paranasal sinuses. Int J Radiat Oncol Biol Phys 67:151-7, 2007

75. Madani I, Bonte K, Vakaet L, et al: Intensity-modulated radiotherapy for sinonasal tumors: Ghent University Hospital update. Int J Radiat Oncol Biol Phys 73:424-32, 2009

76. Weber DC, Chan AW, Lessell S, et al: Visual outcome of accelerated fractionated radiation for advanced sinonasal malignancies employing photons/protons. Radiother Oncol 81:243-9, 2006

77. Mizoe JE, Hasegawa A, Bessho H, et al: Phase II Clinical Trial for Mucosal Malignant Melanoma of the Head and Neck Combined with Chemotherapy (Protocol 0007), Proceedings of NIRS-ETOILE: Joint symposium 2009 on carbon ion radiotherapy. Lyon, 2009, pp 35-37

78. Andersen LJ, Berthelsen A, Hansen HS: Malignant melanoma of the upper respiratory tract and the oral cavity. J Otolaryngol 21:180-5, 1992

79. Bridger AG, Smee D, Baldwin MA, et al: Experience with mucosal melanoma of the nose and paranasal sinuses. ANZ J Surg 75:192-7, 2005

80. Gilligan D, Slevin NJ: Radical radiotherapy for 28 cases of mucosal melanoma in the nasal cavity and sinuses. Br J Radiol 64:1147-50, 1991

81. Guzzo M, Grandi C, Licitra L, et al: Mucosal malignant melanoma of head and neck: forty-eight cases treated at Istituto Nazionale Tumori of Milan. Eur J Surg Oncol 19:316-9, 1993

82. Huang SF, Liao CT, Kan CR, et al: Primary mucosal melanoma of the nasal cavity and paranasal sinuses: 12 years of experience. J Otolaryngol 36:124-9, 2007

83. Kingdom TT, Kaplan MJ: Mucosal melanoma of the nasal cavity and paranasal sinuses. Head Neck 17:184-9, 1995

84. Krengli M, Masini L, Kaanders $\mathrm{JH}$, et al: Radiotherapy in the treatment of mucosal melanoma of the upper aerodigestive tract: analysis of 74 cases. A Rare Cancer Network study. Int J Radiat Oncol Biol Phys 65:751-9, 2006

85. Loree TR, Mullins AP, Spellman J, et al: Head and neck mucosal melanoma: a 32-year review. Ear Nose Throat J 78:372-5, 1999

86. Lund VJ, Howard DJ, Harding L, et al: Management options and survival in malignant melanoma of the sinonasal mucosa. Laryngoscope 109:208-11, 1999 
87. Manolidis S, Donald PJ: Malignant mucosal melanoma of the head and neck: review of the literature and report of 14 patients. Cancer 80:1373-86, 1997

88. Meleti M, Leemans CR, de Bree R, et al: Head and neck mucosal melanoma: experience with 42 patients, with emphasis on the role of postoperative radiotherapy. Head Neck 30:1543-51, 2008

89. Nakashima JP, Viegas CM, Fassizoli AL, et al: Postoperative adjuvant radiation therapy in the treatment of primary head and neck mucosal melanomas. ORL J Otorhinolaryngol Relat Spec 70:344-51, 2008

90. Owens JM, Roberts DB, Myers JN: The role of postoperative adjuvant radiation therapy in the treatment of mucosal melanomas of the head and neck region. Arch Otolaryngol Head Neck Surg 129:864-8, 2003

91. Patel SG, Prasad ML, Escrig M, et al: Primary mucosal malignant melanoma of the head and neck. Head Neck 24:247-57, 2002

92. Shibuya $H$, Takeda $M$, Matsumoto $S$, et al: The efficacy of radiation therapy for a malignant melanoma in the mucosa of the upper jaw: an analytic study. Int $\mathrm{J}$ Radiat Oncol Biol Phys 25:35-9, 1993

93. Temam S, Mamelle $G$, Marandas $P$, et al: Postoperative radiotherapy for primary mucosal melanoma of the head and neck. Cancer 103:313-9, 2005

94. Wagner M, Morris CG, Werning JW, et al: Mucosal melanoma of the head and neck. Am J Clin Oncol 31:43-8, 2008

95. Yii NW, Eisen T, Nicolson M, et al: Mucosal malignant melanoma of the head and neck: the Marsden experience over half a century. Clin Oncol (R Coll Radiol) 15:199-204, 2003

96. Yanagi T, Mizoe JE, Hasegawa A, et al: Mucosal Malignant Melanoma of the Head and Neck Treated by Carbon Ion Radiotherapy. Int J Radiat Oncol Biol Phys 74:15-20, 2009

97. Schulz-Ertner D, Nikoghosyan A, Didinger B, et al: Therapy strategies for locally advanced adenoid cystic carcinomas using modern radiation therapy techniques. Cancer 104:338-44, 2005

98. Munter M, Umathum V, Nikoghosyan A, et al: Combination of intensity modulated radiation therapy (IMRT) and a carbon ion boost for subtotal resected or inoperable adenoid cystic carcinomas (ACC's) of the head and neck region, PTCOG 48. Heidelberg, 2009, pp 38

99. Pommier P, Liebsch NJ, Deschler DG, et al: Proton beam radiation therapy for skull base adenoid cystic carcinoma. Arch Otolaryngol Head Neck Surg 132:1242-9, 2006

100. Agarwal JP, Jain S, Gupta T, et al: Intraoral adenoid cystic carcinoma: prognostic factors and outcome. Oral Oncol 44:986-93, 2008

101. Avery CM, Moody AB, McKinna FE, et al: Combined treatment of adenoid cystic carcinoma of the salivary glands. Int J Oral Maxillofac Surg 29:277-9, 2000

102. Bhattacharyya N, Fried MP: Determinants of survival in parotid gland carcinoma: a populationbased study. Am J Otolaryngol 26:39-44, 2005

103. Da Cruz Perez DE, Pires FR, Lopes MA, et al: Adenoid cystic carcinoma and mucoepidermoid carcinoma of the maxillary sinus: report of a 44-year experience of 25 cases from a single institution. J Oral Maxillofac Surg 64:1592-7, 2006

104.Garden AS, Weber RS, Morrison WH, et al: The influence of positive margins and nerve invasion in adenoid cystic carcinoma of the head and neck treated with surgery and radiation. Int J Radiat Oncol Biol Phys 32:619-26, 1995

105. Gomez DR, Hoppe BS, Wolden SL, et al: Outcomes and prognostic variables in adenoid cystic carcinoma of the head and neck: a recent experience. Int J Radiat Oncol Biol Phys 70:1365-72, 2008

106. Gurney TA, Eisele DW, Weinberg V, et al: Adenoid cystic carcinoma of the major salivary glands treated with surgery and radiation. Laryngoscope 115:1278-82, 2005

107. Harbo G, Bundgaard T, Pedersen D, et al: Prognostic indicators for malignant tumours of the parotid gland. Clin Otolaryngol Allied Sci 27:512-6, 2002

108. Hosokawa $\mathrm{Y}$, Ohmori K, Kaneko M, et al: Analysis of adenoid cystic carcinoma treated by radiotherapy. Oral Surg Oral Med Oral Pathol 74:251-5, 1992 
109. Hsu HC, Huang EY, Wang CJ: Postoperative adjuvant radiotherapy for adenoid cystic carcinoma of the head and neck: treatment results and prognostic factors. Chang Gung Med J 26:64653,2003

110. Huber PE, Debus J, Latz D, et al: Radiotherapy for advanced adenoid cystic carcinoma: neutrons, photons or mixed beam? Radiother Oncol 59:161-7, 2001

111. Iseli TA, Karnell LH, Graham SM, et al: Role of radiotherapy in adenoid cystic carcinoma of the head and neck. J Laryngol Otol:1-8, 2009

112. Khan AJ, DiGiovanna MP, Ross DA, et al: Adenoid cystic carcinoma: a retrospective clinical review. Int J Cancer 96:149-58, 2001

113. Kokemueller H, Eckardt A, Brachvogel P, et al: Adenoid cystic carcinoma of the head and neck-a 20 years experience. Int J Oral Maxillofac Surg 33:25-31, 2004

114. Leonetti JP, Marzo SJ, Agarwal N: Adenoid cystic carcinoma of the parotid gland with temporal bone invasion. Otol Neurotol 29:545-8, 2008

115. Mendenhall WM, Morris CG, Amdur RJ, et al: Radiotherapy alone or combined with surgery for adenoid cystic carcinoma of the head and neck. Head Neck 26:154-62, 2004

116. Rapidis AD, Givalos N, Gakiopoulou H, et al: Adenoid cystic carcinoma of the head and neck. Clinicopathological analysis of 23 patients and review of the literature. Oral Oncol 41:328-35, 2005

117. Rhee CS, Won TB, Lee $\mathrm{CH}$, et al: Adenoid cystic carcinoma of the sinonasal tract: treatment results. Laryngoscope 116:982-6, 2006

118. Silverman DA, Carlson TP, Khuntia D, et al: Role for postoperative radiation therapy in adenoid cystic carcinoma of the head and neck. Laryngoscope 114:1194-9, 2004

119. Spiro RH, Huvos AG: Stage means more than grade in adenoid cystic carcinoma. Am J Surg 164:623-8, 1992

120. Takagi D, Fukuda S, Furuta Y, et al: Clinical study of adenoid cystic carcinoma of the head and neck. Auris Nasus Larynx 28 Suppl:S99-102, 2001

121. Therkildsen MH, Christensen M, Andersen LJ, et al: Salivary gland carcinomas--prognostic factors. Acta Oncol 37:701-13, 1998

122. Amirul Islam M, Yanagi T, Mizoe JE, et al: Comparative study of dose distribution between carbon ion radiotherapy and photon radiotherapy for head and neck tumor. Radiat Med 26:41521, 2008

123. Cozzi L, Fogliata A, Lomax A, et al: A treatment planning comparison of 3D conformal therapy, intensity modulated photon therapy and proton therapy for treatment of advanced head and neck tumours. Radiother Oncol 61:287-97, 2001

124. Johansson J, Blomquist E, Montelius A, et al: Potential outcomes of modalities and techniques in radiotherapy for patients with hypopharyngeal carcinoma. Radiother Oncol 72:129-38, 2004

125. Mock U, Georg D, Bogner J, et al: Treatment planning comparison of conventional, 3D conformal, and intensity-modulated photon (IMRT) and proton therapy for paranasal sinus carcinoma. Int J Radiat Oncol Biol Phys 58:147-54, 2004

126. Steneker M, Lomax A, Schneider U: Intensity modulated photon and proton therapy for the treatment of head and neck tumors. Radiother Oncol 80:263-7, 2006

127. Taheri-Kadkhoda Z, Bjork-Eriksson T, Nill S, et al: Intensity-modulated radiotherapy of nasopharyngeal carcinoma: a comparative treatment planning study of photons and protons. Radiat Oncol 3:4, 2008

128. Van de Water T, Lomax A, Bijl H, et al: Comparative treatment planning study between scanned intensity modulated proton therapy and photon therapy in complex oropharyngeal carcinoma, ESTRO. Göteborg, 2008

129. Widesott L, Pierelli A, Fiorino C, et al: Intensity-modulated proton therapy versus helical tomotherapy in nasopharynx cancer: planning comparison and NTCP evaluation. Int J Radiat Oncol Biol Phys 72:589-96, 2008

130. Schilstra C, Meertens $\mathrm{H}$ : Calculation of the uncertainty in complication probability for various dose-response models, applied to the parotid gland. Int J Radiat Oncol Biol Phys 50:147-58, 2001 
131. Semenenko VA, Li XA: Lyman-Kutcher-Burman NTCP model parameters for radiation pneumonitis and xerostomia based on combined analysis of published clinical data. Phys Med Biol 53:737-55, 2008

132. Eisbruch A, Ten Haken RK, Kim HM, et al: Dose, volume, and function relationships in parotid salivary glands following conformal and intensity-modulated irradiation of head and neck cancer. Int J Radiat Oncol Biol Phys 45:577-87, 1999

133. Roesink JM, Moerland MA, Hoekstra A, et al: Scintigraphic assessment of early and late parotid gland function after radiotherapy for head-and-neck cancer: a prospective study of dose-volume response relationships. Int J Radiat Oncol Biol Phys 58:1451-60, 2004

134. Krengli M, Jereczek-Fossa BA, Kaanders $\mathrm{JH}$, et al: What is the role of radiotherapy in the treatment of mucosal melanoma of the head and neck? Crit Rev Oncol Hematol 65:121-8, 2008

135. Overgaard $\mathrm{J}$ : The role of radiotherapy in recurrent and metastatic malignant melanoma: a clinical radiobiological study. Int J Radiat Oncol Biol Phys 12:867-72, 1986

136. Blettner M, Sauerbrei W, Schlehofer B, et al: Traditional reviews, meta-analyses and pooled analyses in epidemiology. Int J Epidemiol 28:1-9, 1999

137. National Institute for Health and Clinical Excellence. Guide to the methods of technology appraisal. London: National Institute for Health and Clinical Excellence 2008.

138.PTCOG: Statistics of patients treated in hadron therapy centers worldwide Accessed on http://ptcog.web.psi.ch/patient_statistics.html

139. Sayed SI, Elmiyeh B, Rhys-Evans P, et al: Quality of life and outcomes research in head and neck cancer: A review of the state of the discipline and likely future directions. Cancer Treat Rev 35:397-402, 2009

140. Goitein M: Trials and tribulations in charged particle radiotherapy. Radiother Oncol 95:23-31, 2010 


\section{Appendix 3.1: Detailed overview of the search queries}

Search query 1: Particle therapy studies

\section{Patient}

\#1 "Salivary gland" OR "Oropharyngeal" OR "Oropharynx" OR "nasopharynx" OR "Nasopharyngeal" OR "Paranasal Sinus" OR "paranasal" OR "hypopharynx" OR "pharynx" OR "oral cavity"

\#2 (Neoplasm ${ }^{\star}$ OR "cancer" OR "tumor" OR "tumour" OR (malignan*) OR (oncol*) OR (carcinoma*) OR (Head and Neck Neoplasms [mesh])

\#3 \#1 AND \#2

\#4 (Carcinoma, Adenoid Cystic/*radiotherapy [mesh]) OR (Neoplasms, Squamous Cell [mesh]) OR (melanoma [mesh]) OR "Adenoid Cystic" OR "Squamous Cell" OR "melanoma" OR "cancer" OR (neoplasm*) OR "tumor" OR "tumour" OR (malignan*) OR (oncol*)

\#5 (("cancer" OR (neoplasm*) OR "tumor" OR "tumour" OR (malignan*) OR (oncol*) OR (carcinoma*)) AND Head Neck) OR (Head and Neck Neoplasms [mesh])

\#6 \#4 AND \#5

\#7 ((Head and Neck Neoplasms [mesh]) OR (Salivary Gland Neoplasms [mesh]) OR (Oropharyngeal Neoplasms [mesh]) OR (Nasopharyngeal Neoplasms [mesh]) OR (Pharyngeal Neoplasms [mesh]) OR (Paranasal Sinus Neoplasms $[\mathrm{mesh}]))$

\#8 \#3 OR \#6 OR \#7

\section{Intervention and Comparison}

\#9 (proton therap*) OR "proton"

\#10 "particle" OR (heavy ion*) OR (light ion*) OR "hadron" OR "carbon"

\#11 "beam irradiation" OR "radiation" OR "irradiation" OR "beam radiation" OR (radiotherap*) OR (beam therap*)

\#12 \#11 OR (ion therap*)

\#13 \#9 AND \#11

\#14 \#10 AND \#12

$\# 15$ (Protons/*therapeutic use [mesh]) OR (Carbon Radioisotopes/*therapeutic use [mesh])

\#16 \#13 OR\#14 OR\#15

\section{Outcome}

\#17 "Mortality" OR (adverse effect*) OR (adverse event*) OR (side effect* $\left.{ }^{\star}\right)$ OR (toxicit*) OR (xerostomi $)$ OR "Disease free survival" OR "overall survival" OR "local control" OR "tumor control" OR "tumour control"

\#18 (Mortality [mesh]) OR (Head and Neck Neoplasms/*mortality [mesh]) OR (Treatment Outcome [mesh]) OR ("Outcome Assessment (Health Care)" [mesh]) OR (adverse effects [subheading]) OR (radiotherapy/adverse effects [mesh]) OR (Sickness Impact Profile [mesh]) OR (Survival Rate [mesh]) OR ("Xerostomia" [mesh]) OR (Disease free survival [mesh])

\#19 \#17 OR \#18

PICO = \#8 AND \#16 AND \#19 


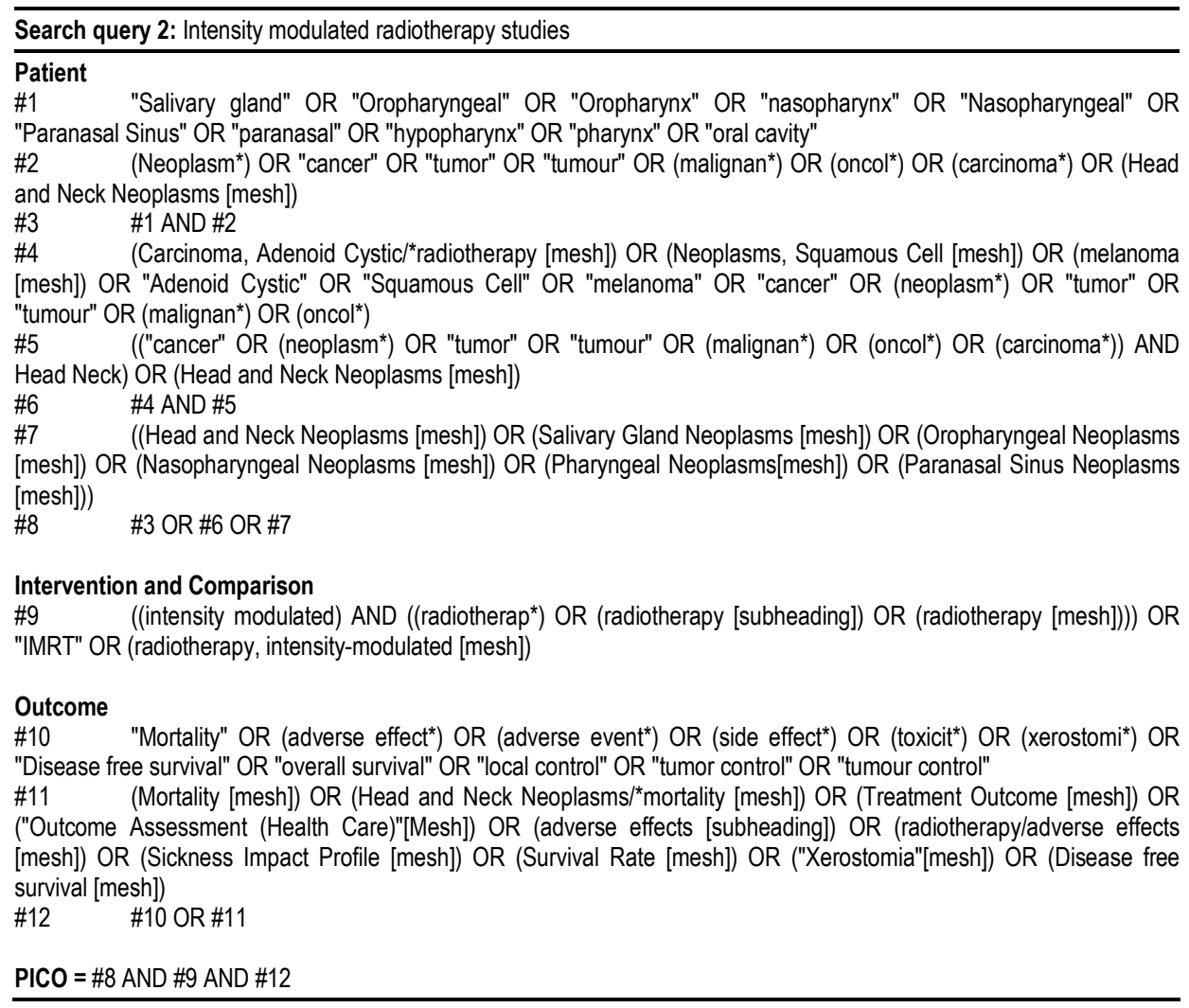




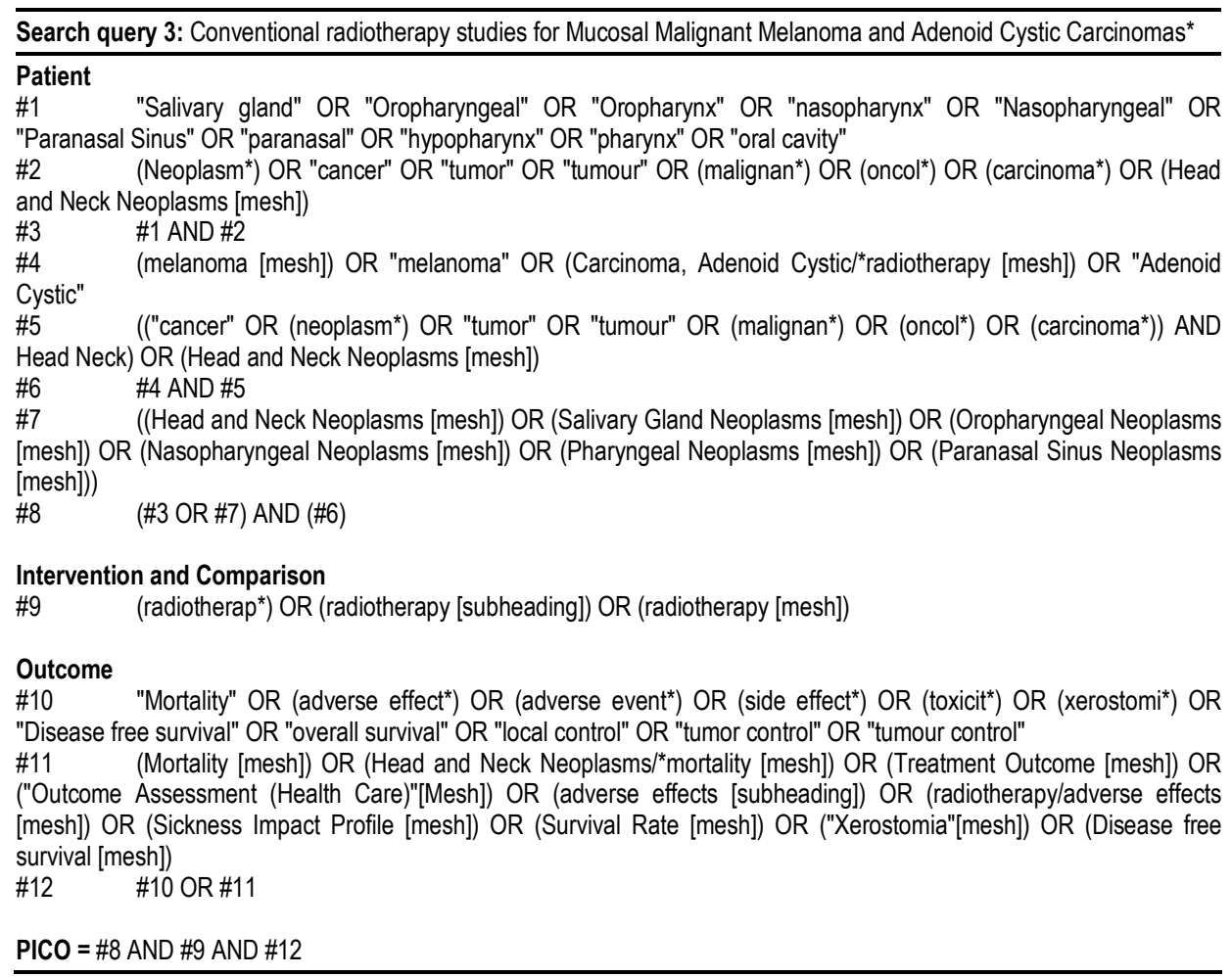

${ }^{*}$ For Mucosal Malignant Melanoma and Adenoid Cystic Carcinomas, no studies using intensity modulated radiotherapy were available 


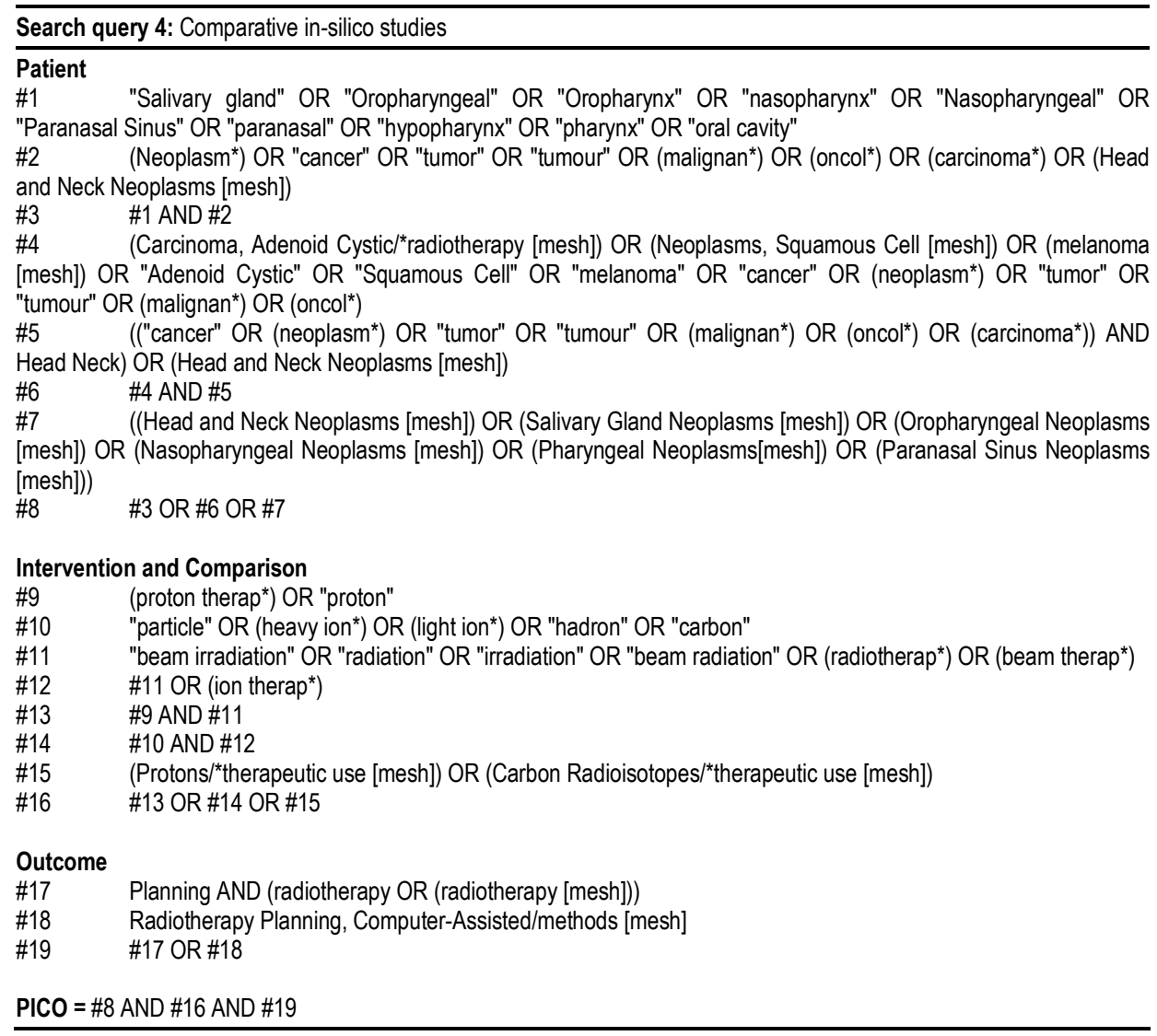


Appendix 3.2: Flow chart of the inclusion and exclusion process for the search strategies

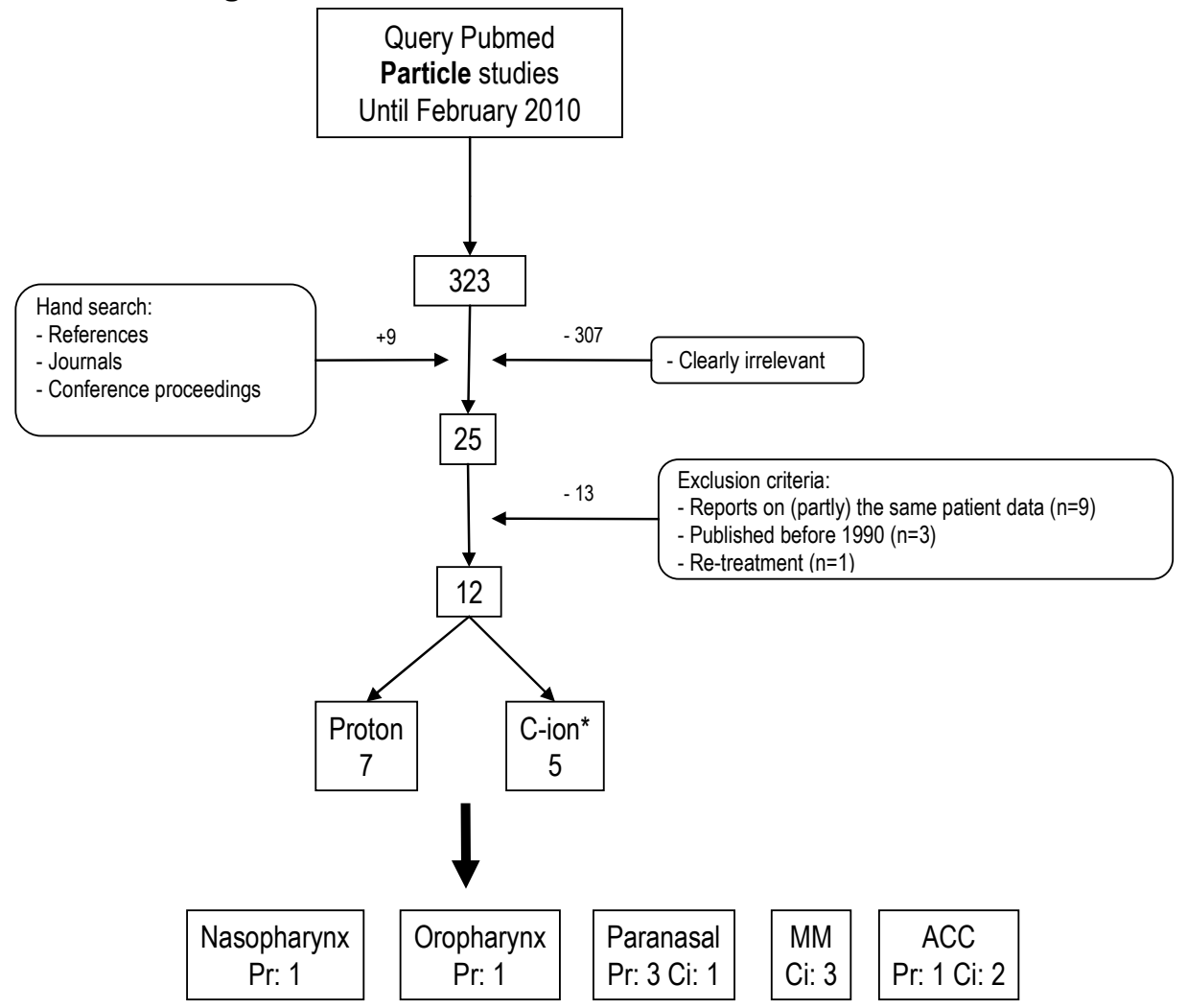

Abbreviations: $\operatorname{Pr}=$ Proton therapy, $\mathrm{Ci} / \mathrm{C}$-ions $=$ carbon-ion therapy, $\mathrm{MM}=$ mucosal malignant melanomas, $\mathrm{ACC}=$ adenoid cystic carcinomas

* Data/sub-analysis extracted from two carbon-ion studies were used in multiple subgroups 


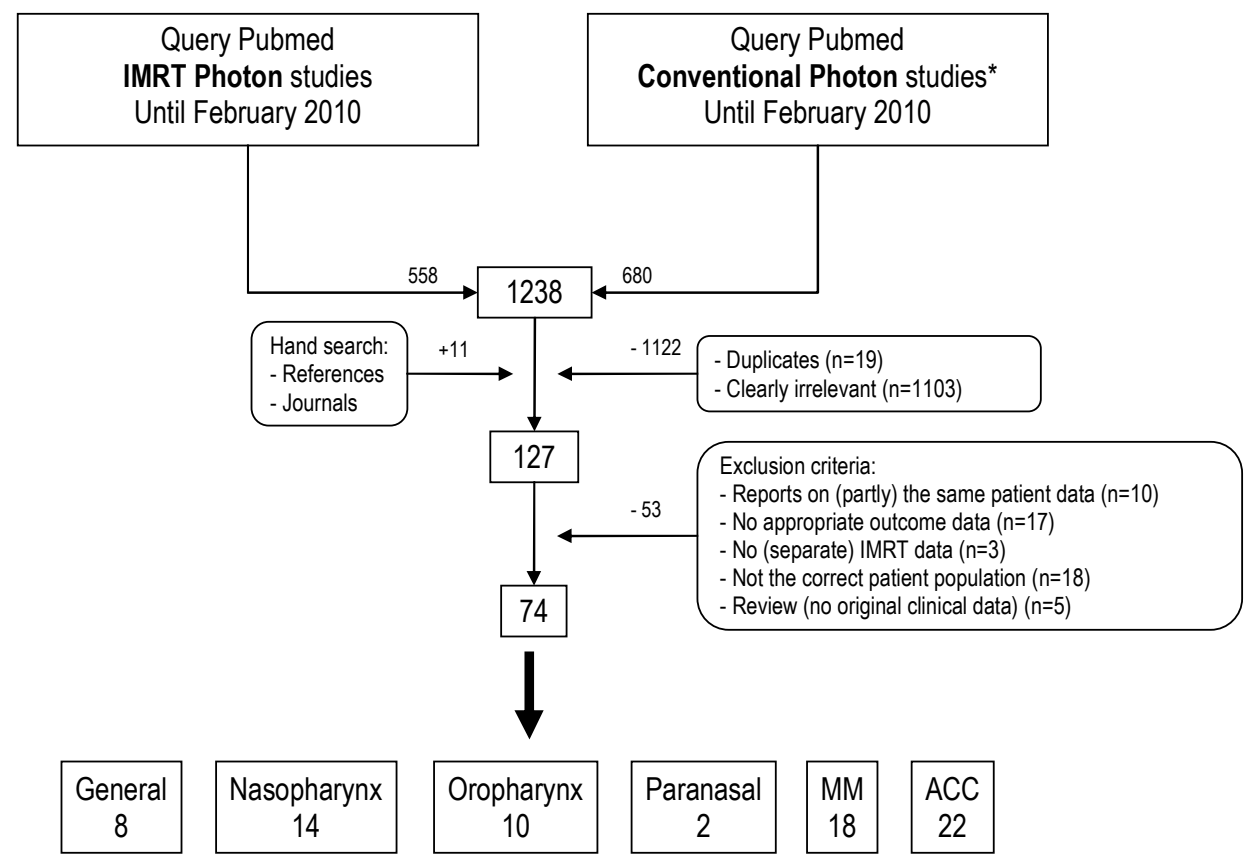

Abbreviations: IMRT $=$ intensity modulated radiotherapy, $\mathrm{MM}=$ mucosal malignant melanomas, $\mathrm{ACC}=$ adenoid cystic carcinomas

* Search query specified for mucosal malignant melanomas and adenoid cystic carcinomas (for these subgroups no IMRT studies were available). 


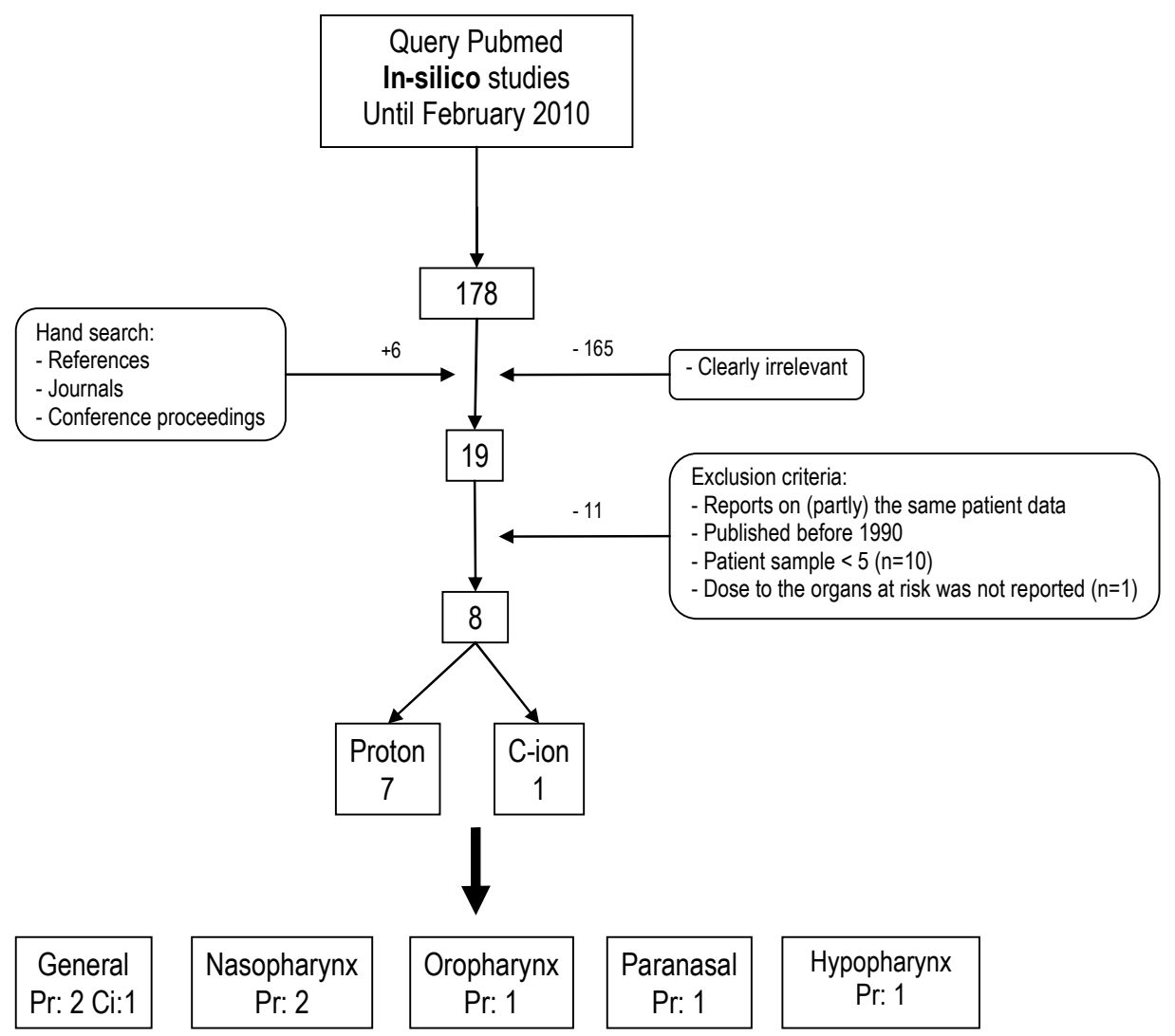

Abbreviations: $\mathrm{Pr}=$ Proton therapy, $\mathrm{Ci} / \mathrm{C}$-ion $=$ carbon-ion therapy, $\mathrm{ACC}=$ adenoid cystic carcinomas 


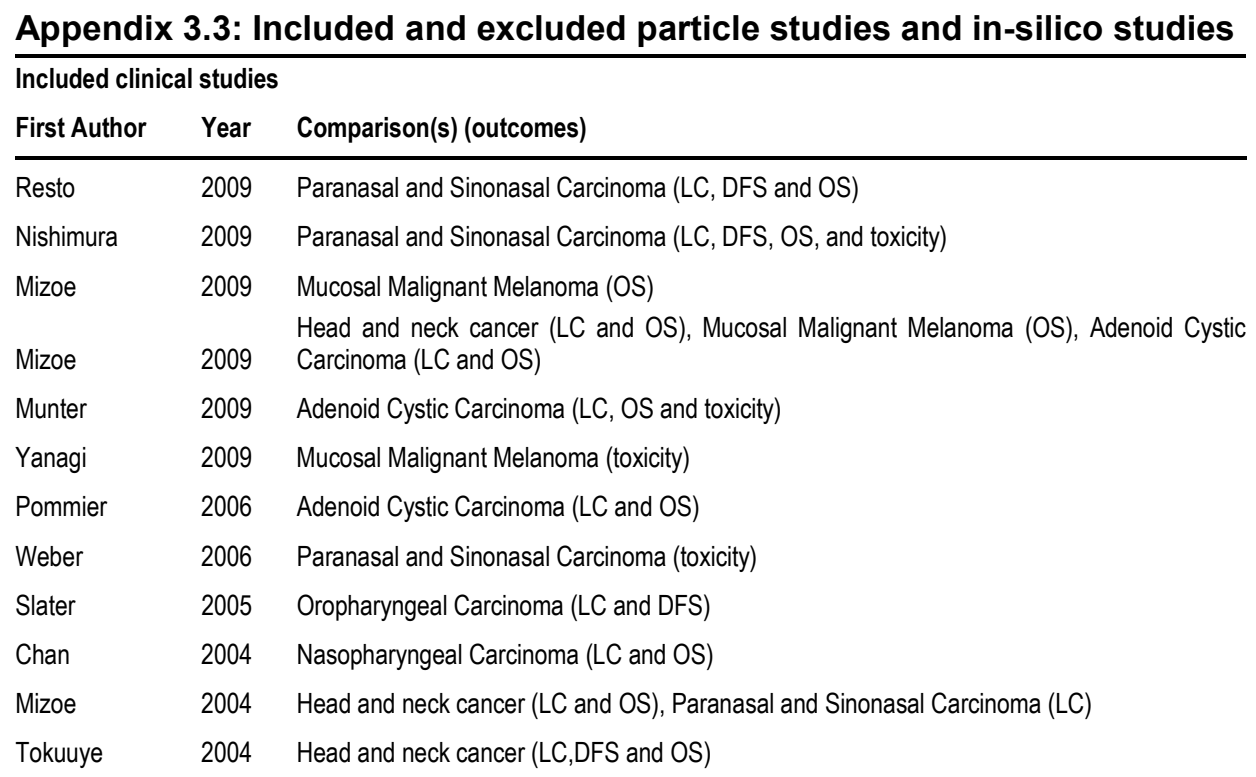

\section{Excluded clinical studies}

\begin{tabular}{lll} 
First Author & Year & Reason(s) for exclusion \\
\hline Truong & 2009 & Overlap in patient sample (with Resto et al, 2008) \\
Takagi & 2007 & Overlap in patient sample (with Mizoe et al, 2009) \\
Chan & 2004 & Overlap in patient sample (with Resto et al, 2008) \\
Schulz-Ertner & 2005 & Overlap in patient sample (with Munter et al, 2009) \\
Schulz-Ertner & 2004 & Overlap in patient sample (with Munter et al, 2009) \\
Schulz-Ertner & 2003 & Overlap in patient sample (with Munter et al, 2009) \\
Fitzek & 2002 & Overlap in patient sample (with Resto et al, 2008) \\
Lin & 1999 & Re-treatment \\
Thornton & 1998 & Overlap in patient sample (with Resto et al, 2008) \\
Tsujii & 1992 & Overlap in patient sample (with Tokuuye et al, 2004) \\
Castro & 1988 & Published before 1990 and other particles than protons and carbon-ions only (including He, C, \\
Munzenrider & 1985 & Published before 1990 \\
Castro & 1982 & Published before 1990 \\
\hline
\end{tabular}




\section{Appendix 3.3 (continued): Included and excluded particle studies and in- silico studies}

\begin{tabular}{llll}
\multicolumn{3}{l}{ Included comparative in-silico studies } \\
First Author & Year & Tumor site/histology \\
\hline Amirul Islam & 2008 & In close proximity of the optic pathways \\
Taheri-Kadkhoda 2008 & Nasopharynx \\
Van de Water & 2008 & Oropharynx \\
Widesott & 2008 & Nasopharynx \\
Steneker & 2006 & Squamous cell carcinoma \\
Johansson & 2004 & Hypopharynx \\
Mock & 2004 & Paranasal sinus \\
Cozzi & 2001 & Squamous cell carcinoma \\
Amirul Islam & 2008 & In close proximity of the optic pathways \\
& & \\
Excluded comparative in-silico studies \\
First Author & Year & Reason(s) for exclusion \\
\hline Chera & 2009 & Patients sample $<5$ \\
Thorwarth & 2008 & Patients sample $<5$ \\
Muzik & 2008 & Patients sample $<5$ \\
Flynn & 2008 & Patients sample $<5$ \\
Lomax & 2003 & Patients sample $<5$ \\
Schulz-Ertner & 2003 & Dose to the organs at risk was not reported \\
Lomax & 1999 & Patients sample $<5$ and no comparison of particle therapy and photons \\
Lomax & 1999 & Patients sample $<5$ \\
Slater & 1992 & Patients sample $<5$ \\
Mirabell & 1992 & Patients sample $<5$ \\
Brown & 1989 & Patients sample $<5$ and published before 1990 \\
\hline & &
\end{tabular}


Appendix 3.4A: Characteristics of nasopharyngeal and oropharyngeal studies*

\begin{tabular}{|c|c|c|c|c|c|c|c|c|}
\hline $\mathbf{N}$ & Modality & $\begin{array}{l}\text { Number of } \\
\text { fractions }^{\dagger}\end{array}$ & $\begin{array}{l}\text { Total } \\
\text { Treatment } \\
\text { dose }(G y E)^{\dagger}\end{array}$ & $\begin{array}{r}\text { Age }^{+} \% \\
\mathrm{Ct}\end{array}$ & $\begin{array}{l}\% \\
\%\end{array} \%$ & $\begin{array}{l}\% \\
\mathrm{~N}+\end{array}$ & $\begin{array}{l}\% \\
\text { N3 }\end{array}$ & $\begin{array}{l}\% \\
\text { Stage IV }\end{array}$ \\
\hline
\end{tabular}

\begin{tabular}{|c|c|c|c|c|c|c|c|c|c|c|c|c|}
\hline \multicolumn{13}{|l|}{ Nasopharynx } \\
\hline Chan $(2004)^{45}$ & 17 & \multicolumn{2}{|c|}{ P-rt + X-rt 46} & 73.6 & 41 & $59 \%$ & $0 \%$ & $100 \%$ & $100 \%$ & $53 \%$ & $6 \%$ & $100 \%$ \\
\hline Zheng (2010) $)^{59}$ & 59 & $X-r t$ & 33 & 70.7 & 48 & $100 \%$ & $\mathrm{nr}$ & $50 \%$ & $28 \%$ & $85 \%$ & $20 \%$ & $43 \%$ \\
\hline Kim $(2009)^{49}$ & 25 & $X-r t$ & 30 & 67.5 & 44 & $100 \%$ & $0 \%$ & $44 \%$ & $24 \%$ & $96 \%$ & $24 \%$ & $48 \%$ \\
\hline Lee $(2009)^{52}$ & 68 & X-rt & 33 & 70.0 & 49 & $84 \%$ & $0 \%$ & $34 \%$ & $19 \%$ & $74 \%$ & $12 \%$ & $28 \%$ \\
\hline $\operatorname{Lin}(2009)^{54}$ & 323 & X-rt & $30 / 31$ & $66.0 / 69.8$ & $\mathrm{nr}$ & $91 \%$ & $0 \%$ & $61 \%$ & $24 \%$ & $91 \%$ & $6 \%$ & $29 \%$ \\
\hline Nishimura $(2009)^{55}$ & 35 & X-rt & $28-35$ & 68.0 & 56 & $89 \%$ & $\mathrm{nr}$ & $46 \%$ & $26 \%$ & $66 \%$ & $11 \%$ & $34 \%$ \\
\hline Tham (2009)56 & 195 & X-rt & 35 & 70.0 & 52 & $56 \%$ & $0 \%$ & $\mathrm{nr}$ & $26 \%$ & $\mathrm{nr}$ & $\mathrm{nr}$ & $30 \%$ \\
\hline Wong (2009) $)^{58}$ & 175 & X-rt & 33 & 70.0 & 48 & $73 \%$ & $0 \%$ & $35 \%$ & $15 \%$ & $72 \%$ & $9 \%$ & $24 \%$ \\
\hline Fang $(2008)^{47}$ & 110 & X-rt & $36-42$ & $64.8-75.6$ & $\mathrm{nr}$ & $57 \%$ & $0 \%$ & $25 \%$ & $11 \%$ & $68 \%$ & $1 \%$ & $12 \%$ \\
\hline Koom $(2008)^{50}$ & 24 & X-rt & 27 & 64.8 & 46 & $100 \%$ & $0 \%$ & $29 \%$ & $13 \%$ & $96 \%$ & $25 \%$ & $33 \%$ \\
\hline Wolden $(2006)^{57}$ & 74 & X-rt & 30 & 70.2 & 45 & $93 \%$ & $0 \%$ & $51 \%$ & $31 \%$ & $78 \%$ & $24 \%$ & $47 \%$ \\
\hline Chong $(2004)^{46}$ & 104 & X-rt & $25-30$ & $64.0-70.0$ & 43 & $\mathrm{nr}$ & $\mathrm{nr}$ & $\mathrm{nr}$ & $\mathrm{nr}$ & $\mathrm{nr}$ & $\mathrm{nr}$ & $16 \%$ \\
\hline Kam $(2004)^{48}$ & 63 & X-rt & 33 & 66.0 & 48 & $30 \%$ & $0 \%$ & $51 \%$ & $19 \%$ & $70 \%$ & $5 \%$ & $22 \%$ \\
\hline Kwong $(2004)^{51}$ & 33 & X-rt & 34 & $68.0-70.0$ & 43 & $0 \%$ & $0 \%$ & $3 \%$ & $0 \%$ & $18 \%$ & $0 \%$ & $0 \%$ \\
\hline Lee $(2002)^{53}$ & 67 & X-rt & $\mathrm{nr}$ & $65.0-70.0$ & 49 & $75 \%$ & $0 \%$ & $43 \%$ & $21 \%$ & $78 \%$ & $12 \%$ & $37 \%$ \\
\hline \multicolumn{13}{|l|}{ Oropharynx } \\
\hline Slater $(2005)^{60}$ & 29 & \multicolumn{2}{|c|}{ P-rt + X-rt 45} & 75.9 & $\mathrm{nr}$ & $0 \%$ & $0 \%$ & $55 \%$ & $21 \%$ & $83 \%$ & $10 \%$ & $62 \%$ \\
\hline Daly $(2010)^{62}$ & 107 & X-rt & 30 & $60.0 / 66.0$ & $\mathrm{nr}$ & $80 \%$ & $21 \%$ & $47 \%$ & $29 \%$ & $91 \%$ & $11 \%$ & $86 \%$ \\
\hline Eisbruch (2009)63 & 67 & X-rt & 30 & 66.0 & 56 & $0 \%$ & $0 \%$ & $0 \%$ & $0 \%$ & $43 \%$ & $0 \%$ & $0 \%$ \\
\hline Lawson $(2008)^{66}$ & 34 & X-rt & 33 & 70.3 & 61 & $100 \%$ & $0 \%$ & $41 \%$ & $29 \%$ & $91 \%$ & $9 \%$ & $85 \%$ \\
\hline Rusthoven (2008)68 & 32 & X-rt & 33 & 69.3 & $\mathrm{nr}$ & $100 \%$ & $0 \%$ & $41 \%$ & $19 \%$ & $97 \%$ & $3 \%$ & $84 \%$ \\
\hline Schoenfeld $(2008)^{69}$ & 964 & X-rt & 42 & $67.5 / 72.0$ & $\mathrm{nr}$ & $54 \% \ddagger$ & $0 \%$ & $42 \% \ddagger$ & $20 \%^{\ddagger}$ & $70 \%^{\ddagger}$ & 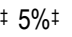 & $63 \%^{\ddagger}$ \\
\hline Garden $(2007)^{64}$ & 51 & X-rt & 30 & $63.0-66.0$ & 54 & $10 \%$ & $0 \%$ & $0 \%$ & $0 \%$ & $67 \%$ & $4 \%$ & $53 \%$ \\
\hline Lee $(2006)^{67}$ & 41 & X-rt & $40 / 42$ & $70.0-72.0$ & 55 & $100 \%$ & $0 \%$ & $39 \%$ & $\mathrm{nr}$ & $90 \%$ & $5 \%$ & $85 \%$ \\
\hline Yao $(2006)^{70}$ & 66 & X-rt & $\mathrm{nr}$ & $60.0-74.0$ & 53 & $70 \%$ & $6 \%$ & $41 \%$ & $29 \%$ & $88 \%$ & $14 \%$ & $88 \%$ \\
\hline Chao $(2004)^{71}$ & 74 & X-rt & 35 & $66.0 / 70.0$ & 55 & $23 \%$ & $58 \%$ & $45 \%$ & $\mathrm{nr}$ & $84 \%$ & $\mathrm{nr}$ & $70 \%$ \\
\hline Huang (2003) & 41 & X-rt & 33 & 70.0 & 55 & $73 \%$ & $7 \%$ & $17 \%$ & $7 \%$ & $76 \%$ & $5 \%$ & $68 \%$ \\
\hline
\end{tabular}


Appendix 3.4B: Characteristics of paranasal and sinonasal and mucosal malignant melanoma studies*

\begin{tabular}{|c|c|c|c|c|c|c|c|c|c|}
\hline & $\mathbf{N}$ & Mod & $\begin{array}{l}\text { Total } \\
\text { treatment } \\
\text { dose (GyE) }\end{array}$ & Age $^{\dagger}$ & $\begin{array}{l}\% \\
\mathrm{Ct}\end{array}$ & $\begin{array}{l}\% \\
\text { Operated } \\
\text { patients }\end{array}$ & $\begin{array}{l}\text { \% } \\
\text { T4 }\end{array}$ & $\begin{array}{l}\% \\
\text { N3 }\end{array}$ & $\begin{array}{l}\text { \% } \\
\text { Stage IIII }\end{array}$ \\
\hline \multicolumn{10}{|c|}{ Paranasal and Sinonasal } \\
\hline Resto Cs $(2008)^{73}$ & 20 & \multicolumn{2}{|c|}{ P-rt + X-rt67.6 } & $50 \ddagger$ & $33 \%^{\ddagger}$ & $100 \%$ & $\mathrm{nr}$ & $\mathrm{nr}$ & na \\
\hline Resto Ps $(2008)^{73}$ & 50 & \multicolumn{2}{|c|}{ P-rt + X-rt75.6 } & $50 \ddagger$ & $33 \% \ddagger$ & $100 \%$ & $\mathrm{nr}$ & $\mathrm{nr}$ & na \\
\hline Resto Bo $(2008)^{73}$ & 32 & \multicolumn{2}{|c|}{ P-rt + X-rt75.4 } & $50 \ddagger$ & $33 \% \ddagger$ & $0 \%$ & $\mathrm{nr}$ & $\mathrm{nr}$ & na \\
\hline Nishimura $(2007)^{72}$ & 14 & P-rt & 65.0 & 56 & $29 \%$ & $50 \%$ & $\mathrm{nr}$ & $\mathrm{nr}$ & na \\
\hline Mizoe $(2004)^{35}$ & 10 & C-rt & $48.6-64.8$ & $60 \ddagger$ & $6 \% \ddagger$ & $33 \%^{\ddagger}$ & $\mathrm{nr}$ & $\mathrm{nr}$ & na \\
\hline Madani $(2009)^{75}$ & 73 & $X-r t$ & 70.0 & 63 & $0 \%$ & $89 \%^{\ddagger}$ & $39 \% \neq$ & $0 \%$ & na \\
\hline Daly $(2007)^{74}$ & 36 & $X-r t$ & 70.0 & 57 & $22 \%$ & $89 \%$ & $69 \%$ & $0 \%$ & na \\
\hline \multicolumn{10}{|c|}{ Mucosal Malignant Melanoma } \\
\hline Mizoe $(2009)^{34}$ & \multicolumn{2}{|c|}{$100 \mathrm{C}-\mathrm{rt}$} & 57.6 & $57^{\ddagger}$ & $0 \%$ & $\mathrm{nr}$ & na & na & $\mathrm{nr}$ \\
\hline Mizoe $+\mathrm{Ct}(2009)^{77}$ & 57 & C-rt & 57.6 & 60 & $100 \%$ & $\mathrm{nr}$ & na & na & $\mathrm{nr}$ \\
\hline Meleti (2008) ${ }^{88}$ & 42 & X-rt & 30.0 & 64 & $2 \%$ & $90 \%$ & na & na & $3 \% \ddagger$ \\
\hline Nakashima $(2008)^{89}$ & 20 & X-rt & 54.0 & 62 & $6 \%$ & $100 \%$ & na & na & $0 \%$ \\
\hline Wagner $(2008)^{94}$ & 17 & X-rt & 66.9 & 64 & $0 \%$ & $76 \%$ & na & na & $0 \%$ \\
\hline Huang $(2007)^{82}$ & 15 & $X-r t$ & $\mathrm{nr}$ & 69 & $20 \%$ & $93 \%$ & na & na & $0 \%$ \\
\hline Krengli (2006) ${ }^{84}$ & 74 & X-rt & 55.1 & 66 & $5 \%$ & $80 \%$ & na & na & $8 \%$ \\
\hline Bridger $(2005)^{79}$ & 27 & $X-r t$ & $\mathrm{nr}$ & 66 & $0 \%$ & $100 \%$ & na & na & $\mathrm{nr}$ \\
\hline Temam $(2005)^{93}$ & 69 & X-rt & 64.9 & 58 & $0 \%$ & $100 \%$ & na & na & $0 \%$ \\
\hline Owens $(2003)^{90}$ & 44 & $X-r t$ & $\mathrm{nr}$ & 56 & $0 \%$ & $100 \%$ & na & na & $\mathrm{nr}$ \\
\hline Yii $(2003)^{95}$ & 89 & X-rt & $\mathrm{nr}$ & 64 & $10 \%$ & $97 \%$ & na & na & $7 \%$ \\
\hline Patel $(2002)^{91}$ & 59 & $X-r t$ & $\mathrm{nr}$ & 63 & $0 \%$ & $100 \%$ & na & na & $7 \%$ \\
\hline Loree $(1999)^{85}$ & 28 & X-rt & $\mathrm{nr}$ & $\mathrm{nr}$ & $18 \%$ & $61 \%$ & na & na & $4 \%$ \\
\hline Lund (1999) ${ }^{86}$ & 58 & $X-r t$ & $\mathrm{nr}$ & 64 & $16 \%$ & $100 \%$ & na & na & $0 \%$ \\
\hline Manolidis $(1997)^{87}$ & 14 & $X-r t$ & $\mathrm{nr}$ & 71 & $0 \%$ & $86 \%$ & na & na & $\mathrm{nr}$ \\
\hline Kingdom (1995) & 10 & $X-r t$ & $\mathrm{nr}$ & $\mathrm{nr}$ & $0 \%$ & $100 \%$ & na & na & $\mathrm{nr}$ \\
\hline Guzzo (1993) ${ }^{81}$ & 48 & X-rt & $\mathrm{nr}$ & 58 & $29 \%$ & $90 \%$ & na & na & $8 \%$ \\
\hline Shibuya $(1993)^{92}$ & 28 & X-rt & $\mathrm{nr}$ & $\mathrm{nr}$ & $0 \%$ & $0 \%$ & na & na & $0 \%$ \\
\hline Andersen $(1992)^{78}$ & 26 & X-rt & 54.0 & 65 & $0 \%$ & $77 \%$ & na & na & $4 \%$ \\
\hline Gilligan $(1991)^{80}$ & 28 & $X-r t$ & $45.0-55.0$ & 65 & $0 \%$ & $0 \%$ & na & na & $\mathrm{nr}$ \\
\hline
\end{tabular}


Appendix 3.4C: Characteristics of adenoid cystic carcinoma studies*

\begin{tabular}{|c|c|c|c|c|c|c|c|c|c|}
\hline & $\mathbf{N}$ & Modality & $\begin{array}{l}\text { Total } \\
\text { treatment } \\
\text { dose }(G y E) \dagger\end{array}$ & Aget $^{\dagger}$ & $\begin{array}{l}\% \\
\mathrm{Ct}\end{array}$ & $\begin{array}{l}\% \\
\text { Operated } \\
\text { patients }\end{array}$ & $\begin{array}{l}\% \\
\mathrm{~T} 4\end{array}$ & $\begin{array}{l}\% \\
\text { N3 }\end{array}$ & $\begin{array}{l}\% \\
\text { Stage IV }\end{array}$ \\
\hline \multicolumn{10}{|c|}{ Adenoid Cystic Carcinoma } \\
\hline Pommier $(2006)^{99}$ & 23 & P-rt + X-rt & $73.3 / 76.4$ & 46 & $4 \%$ & $52 \%$ & $\mathrm{nr}$ & $0 \%$ & $\mathrm{nr}$ \\
\hline Mizoe (2009) $)^{34}$ & 107 & C-rt & 57.6 & $57 \ddagger$ & $\mathrm{nr}$ & $\mathrm{nr}$ & $\mathrm{nr}$ & $\mathrm{nr}$ & $\mathrm{nr}$ \\
\hline Munter (2009) ${ }^{98}$ & 59 & C-rt + X-rt & 72.0 & 52 & $\mathrm{nr}$ & $\mathrm{nr}$ & $\mathrm{nr}$ & $\mathrm{nr}$ & $\mathrm{nr}$ \\
\hline Iseli (2009) $)^{111}$ & 171 & X-rt & $60.0-70.0$ & 53 & $0 \%$ & $82 \%$ & $\mathrm{nr}$ & $\mathrm{nr}$ & $37 \%$ \\
\hline Agarwal $(2008)^{100}$ & 76 & X-rt & 56.0 & $\mathrm{nr}$ & $0 \%$ & $97 \%$ & $54 \%$ & $\mathrm{nr}$ & $59 \%$ \\
\hline Gomez $(2008)^{105}$ & 59 & X-rt & 63.0 & 52 & $10 \%$ & $92 \%$ & $34 \%$ & $\mathrm{nr}$ & $\mathrm{nr}$ \\
\hline Leonetti (2008) $)^{114}$ & 16 & X-rt & $51.0-60.0$ & 47 & $0 \%$ & $100 \%$ & $\mathrm{nr}$ & $\mathrm{nr}$ & $\mathrm{nr}$ \\
\hline Da Cruz Perez (2006) ${ }^{103}$ & 18 & X-rt & 53.6 & 39 & $17 \%$ & $61 \%$ & $\mathrm{nr}$ & $\mathrm{nr}$ & $\mathrm{nr}$ \\
\hline Rhee $(2006)^{117}$ & 35 & X-rt & $\mathrm{nr}$ & 47 & $9 \%$ & $77 \%$ & $49 \%$ & $\mathrm{nr}$ & $\mathrm{nr}$ \\
\hline Bhattacharyya (2005) ${ }^{102}$ & 18 & X-rt & $\mathrm{nr}$ & $59 \ddagger$ & $\mathrm{nr}$ & $\mathrm{nr}$ & $\mathrm{nr}$ & $\mathrm{nr}$ & $\mathrm{nr}$ \\
\hline Gurney (2005) & 33 & X-rt & 60.0 & 49 & $0 \%$ & $100 \%$ & $\mathrm{nr}$ & $\mathrm{nr}$ & $31 \%$ \\
\hline Rapidis (2005) $)^{116}$ & 23 & X-rt & $\mathrm{nr}$ & 60 & $26 \%$ & $96 \%$ & $\mathrm{nr}$ & $\mathrm{nr}$ & $\mathrm{nr}$ \\
\hline Kokemeuller (2004) $)^{113}$ & 74 & X-rt & 60.0 & 58 & $0 \%$ & $100 \%$ & $42 \%$ & $0 \%$ & $45 \%$ \\
\hline Mendenhall (2004) & 101 & X-rt & $10.5-79.2$ & 58 & $2 \%$ & $58 \%$ & $44 \%$ & $0 \%$ & $45 \%$ \\
\hline Silverman $(2004)^{118}$ & 75 & X-rt & $\mathrm{nr}$ & 52 & $0 \%$ & $100 \%$ & $31 \%$ & $0 \%$ & $31 \%$ \\
\hline Hsu $(2003)^{109}$ & 25 & X-rt & $\mathrm{nr}$ & 50 & $0 \%$ & $100 \%$ & $\mathrm{nr}$ & $\mathrm{nr}$ & $12 \%$ \\
\hline Harbo $(2002)^{107}$ & 26 & X-rt & $\mathrm{nr}$ & $64 \ddagger$ & $0 \%$ & $49 \% \ddagger$ & $7 \% \ddagger$ & $2 \% \ddagger$ & $36 \% \ddagger$ \\
\hline Huber $(2001)^{110}$ & 25 & X-rt & 64.0 & $55^{\ddagger}$ & $0 \%$ & $68 \%$ & $\mathrm{nr}$ & $\mathrm{nr}$ & $\mathrm{nr}$ \\
\hline Khan $(2001)^{112}$ & 68 & X-rt & $\mathrm{nr}$ & 52 & $3 \%$ & $87 \%$ & $22 \%$ & $\mathrm{nr}$ & $31 \%$ \\
\hline Takagi $(2001)^{120}$ & 20 & X-rt & $40.0-65.0$ & 59 & $35 \%$ & $85 \%$ & $\mathrm{nr}$ & $\mathrm{nr}$ & $\mathrm{nr}$ \\
\hline Avery $(2000)^{101}$ & 15 & X-rt & $\mathrm{nr}$ & 53 & $7 \%$ & $100 \%$ & $\mathrm{nr}$ & $\mathrm{nr}$ & $33 \%$ \\
\hline Therkildsen (1998) ${ }^{121}$ & 87 & X-rt & $60.0-66.0$ & $60 \ddagger$ & $0 \%$ & $91 \%{ }^{\ddagger}$ & $\mathrm{nr}$ & $\mathrm{nr}$ & $17 \% \ddagger$ \\
\hline Garden (1995) ${ }^{104}$ & 198 & X-rt & 60.0 & 50 & $0 \%$ & $100 \%$ & $\mathrm{nr}$ & $\mathrm{nr}$ & $\mathrm{nr}$ \\
\hline Hosokawa (1992) ${ }^{108}$ & 41 & X-rt & $40.0-70.0$ & 58 & $0 \%$ & $79 \%$ & $\mathrm{nr}$ & $\mathrm{nr}$ & $\mathrm{nr}$ \\
\hline Spiro (1992) ${ }^{119}$ & 184 & X-rt & $\mathrm{nr}$ & 57 & $0 \%$ & $95 \%$ & $\mathrm{nr}$ & $\mathrm{nr}$ & $12 \% \ddagger$ \\
\hline
\end{tabular}

Abbreviations: $\mathrm{X}-\mathrm{rt}=$ photon radiotherapy, $\mathrm{P}-\mathrm{rt}=$ proton radiotherapy, $\mathrm{C}-\mathrm{rt}=$ carbon-ion radiotherapy, $\mathrm{Cs}=$ complete surgery, $\mathrm{Ps}=$ partial surgery, $\mathrm{Bo}=$ biopsy only, $\mathrm{Ct}=$ chemotherapy, $\mathrm{nr}=\mathrm{not}$ reported, na $=$ not applicable

* Only characteristics of studies included in meta-analyses were reported

$\uparrow$ Median/mean value

$\mp$ Figures are for the whole study sample not only for this subgroup

$\S$ According to Ballantyne's staging system 



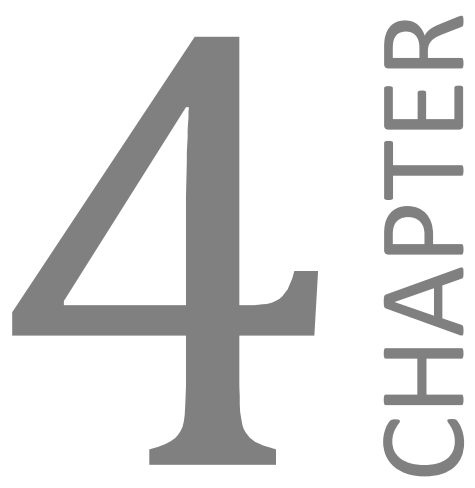

THE IMPACT OF LATE TREATMENT-TOXICITY ON GENERIC HEALTH-RELATED QUALITY OF LIFE IN HEAD AND NECK CANCER PATIENTS AFTER RADIOTHERAPY

\author{
Bram Ramaekers \\ Manuela Joore \\ Janneke Grutters \\ Piet Van Den Ende \\ Jos De Jong \\ Ruud Houben \\ Philippe Lambin \\ Miranda Christianen \\ Ivo Beetz \\ Madelon Pijls-Johannesma \\ Johannes Langendijk
}

Oral Oncology, 2011 Aug;47(8):768-74 


\section{Abstract}

Objective: To examine the impact of late treatment-related xerostomia and dysphagia on health-related quality of life (HRQOL) in head and neck cancer (HNC) patients after radiotherapy.

Methods: A multi-center cross-sectional survey was performed. Patients with a follow-up of at least 6 months after curative radiotherapy, without evidence of recurrent disease were eligible for inclusion. The Euroqol-5D questionnaire (EQ-5D) was filled out and toxicity was scored and converted to the RTOG scale. The EQ-5D measures generic HRQOL in terms of utility and Visual Analogue Scale (VAS) scores. Missing data on the EQ-5D were imputed using multiple imputation.

HRQOL was compared between subgroups of patients with and without toxicity. Subsequently, the impact of xerostomia and dysphagia on HRQOL was analysed using multivariate regression analyses. Both analyses were performed separately for utility scores and VAS scores.

Results: The study population was composed of $396 \mathrm{HNC}$ patients. The average utility and VAS scores were 0.85 (scale 0-1) and 75 (scale 0-100). Subgroups of patients with xerostomia and/or dysphagia showed statistically significantly lower utility and VAS scores $(P=0.000-0.022)$. The multivariate regression model showed that xerostomia and dysphagia were negative predictors of both utility and VAS scores. Other factors which influenced HRQOL in at least one of the two regression models were: sex, tumor location and the addition of surgery to radiotherapy.

Conclusion: Xerostomia and dysphagia diminish generic HRQOL. Moreover dysphagia affects patients' HRQOL stronger than xerostomia.

Keywords: Quality of life; Head and neck cancer; Xerostomia, Dysphagia; Radiotherapy 


\section{Introduction}

An increasing number of options become available for the treatment of head and neck cancer (HNC). Traditionally, treatment decisions were primarily based on local tumor control and the length of survival. However, treating HNC often involves a trade-off between (disease-free) survival and treatment-related toxicity. After radiotherapy, in particular xerostomia and dysphagia negatively affect health-related quality of life (HRQOL). ${ }^{1,2}$ Therefore, the length of survival after treatment has to be weighed against the quality of survival. As a result, $\mathrm{HRQOL}$ and factors influencing HRQOL (such as treatment-related toxicity) are increasingly recognized as important treatment outcomes in $\mathrm{HNC} .^{1,3-5}$

To take into account HRQOL, disease-specific measures are often used. Although these questionnaires are relevant for patients and physicians, the main disadvantage of disease-specific measures is their inability to compare HRQOL in different disease areas. In contrast, generic HRQOL can be compared in different disease areas. Measures of generic HRQOL assess the preferences of individuals for a certain health status; the more preferable the outcome, the higher the score. ${ }^{6}$ This can be measured by inquiring how patients value their own health status (patients' perspective) or based on the preferences of the society (general public perspective). Preferences for health states can be combined with life expectancy, resulting in Quality Adjusted Life Years (QALYs). The main advantage of QALYs is that they capture life expectancy and quality of life in a single measure. ${ }^{6}$ This is useful to inform the aforementioned trade-off between length of survival and treatment-related toxicity (quality of survival).

Previous studies have assessed health state preferences in HNC. ${ }^{7-11}$ However, to our knowledge, no study provided health state preferences based on subgroups distinguished by treatment-related toxicity for primary treated HNC patients. Hence, knowledge on the impact of toxicity on generic HRQOL is lacking. Therefore, we aimed to examine the impact of late treatment-related xerostomia and dysphagia on HRQOL (patient and general public perspective) in disease free HNC patients treated with radiotherapy.

The aim of this study was twofold: (1) to compare HRQOL across subgroups subdivided by xerostomia and dysphagia and, (2) to examine the influence of late treatment-related toxicity on HRQOL.

\section{Methods}

\section{Conceptual model}

To examine the influence of late treatment-related toxicity on HRQOL, we constructed a conceptual model (Figure 4.1). In order to minimize multicollinear- 
ity in our analyses, we only included factors that are directly related to HRQOL, excluding indirect factors (i.e. variables that influence HRQOL through other variables).

$\mathrm{HRQOL}$ is negatively affected by radiotherapy induced xerostomia and dysphagia. $^{1,2,12}$ Since dysphagia may be the consequence of xerostomia, these outcome measures might correlate or even interact. Next to these main variables of interest, other potentially predictive factors were subdivided into patient related factors, disease related factors and treatment-related factors. Patient related factors include sex and age; HRQOL scores are likely to decrease with increasing age and tend to be lower for females. ${ }^{13-15}$ Disease related factors include tumor location and disease stage. Tumor location has a varying impact on HRQOL. ${ }^{1,2,16,17}$ In contrast, disease stage is unlikely to directly affect HRQOL. ${ }^{1,2,16,17}$ In case of a lower HRQOL for patients with advanced disease stage, the decreased HRQOL is suggested to be related to cancer treatment, treatment toxicity and/or disease progression rather than to cancer stage directly ${ }^{2,16,18}$ Hence, disease stage can be considered as an indirect predictor of $\mathrm{HRQOL}$ and is for this reason not included in our conceptual model. Finally, with regard to treatment-related factors, the combination of radiotherapy with surgery or chemotherapy is suggested to have a negative influence on HRQOL. ${ }^{1,16}$ The interval after radiotherapy (follow-up period) has a varying impact on HRQOL. ${ }^{1,19}$

\section{Data collection}

From June 2009 to March 2010, a multi-center cross-sectional survey was performed during planned follow-up visits in two Dutch hospitals. ${ }^{20}$ The study population consisted of HNC patients, who were treated with curative intend by radiotherapy alone or combined with surgery and/or chemotherapy. Patients with a follow-up period of 6 months or longer after the start of radiotherapy and without evidence of recurrent disease were eligible for inclusion.

The EuroQol-5D questionnaire ${ }^{21}$ (EQ-5D) was filled out in the hospital before the patients visited their physician. During the follow-up visit, severity of xerostomia and dysphagia was scored by a trained researcher (radiation technologist) or the treating physician and converted to the RTOG scale ${ }^{22}$ (Appendix 4.1). The following data were retrieved from patients' medical records: date and type of initial treatment, primary tumor location and initial tumor stage. This study was approved by the Institutional Review Board. 

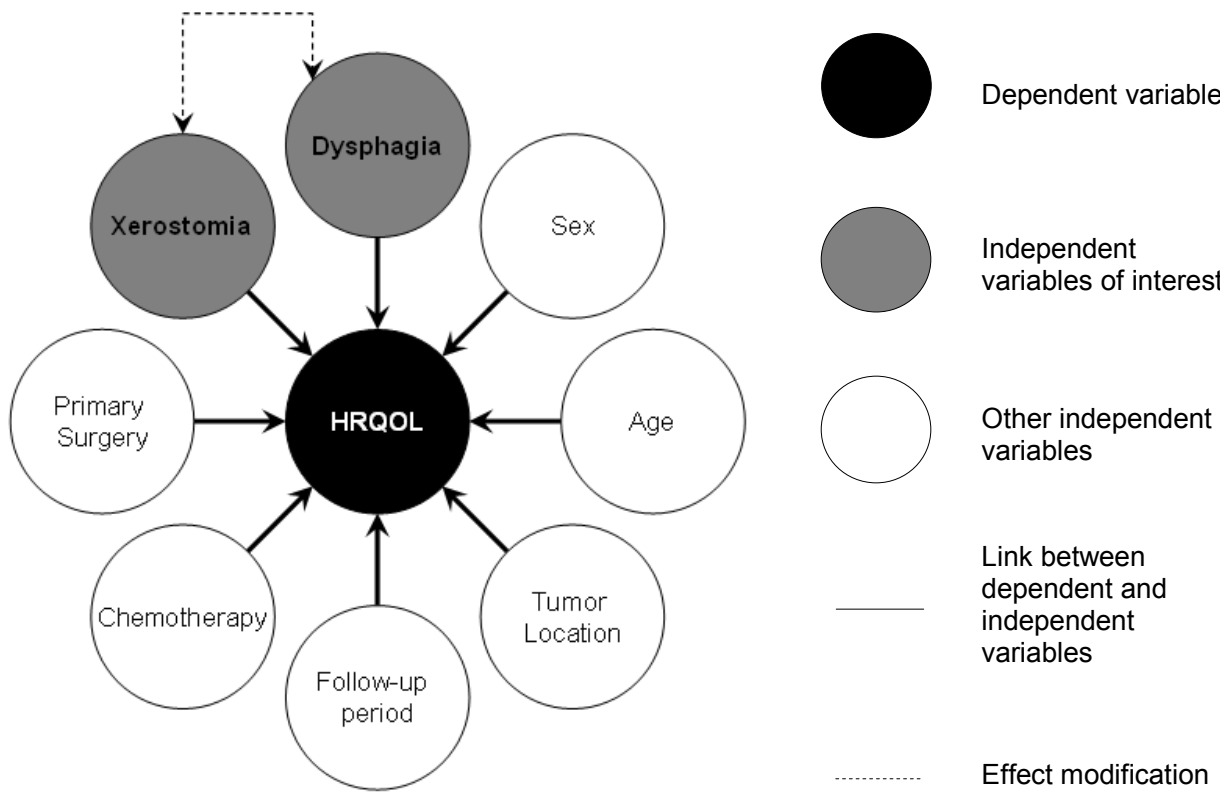

Figure 4.1: Conceptual model

\section{EuroQol-5D}

The EQ-5D is the most frequently used multi-attribute health status classification system and is recommended by the National Institute for Health and Clinical Excellence. ${ }^{6,21,23-25}$ The EQ-5D consists of questions considering five dimensions of generic HRQOL (mobility, self-care, usual activities, pain/discomfort and anxiety/depression). ${ }^{21}$ The answers to these questions can be combined to calculate health state preferences (so-called utility scores) from a general public perspective using a scoring function. This scoring function was based on the preferences of 2.997 UK respondents, who valued 42 different health states from the EQ-5D using the time trade-off method (TTO). ${ }^{26}$ Utility scores calculated by this scoring function range from -0.59 (health state worse than death, severe problems in all five dimensions), through 0 (death) to 1 (full health, no problems in all dimensions). Next to the five questions, a Visual Analogue Scale (VAS) is included in the EQ-5D that ranges from 0 (worst imaginable health state) to 100 (best imaginable health state). ${ }^{21}$ The VAS measures the patients' self-rated HRQOL (patients' perspective). ${ }^{27}$

\section{Data analyses}

\section{Missing data}

Patients with missing data for late toxicity scores were excluded from the analyses. As recommended for handling missing HRQOL data, ${ }^{28}$ missing data on 
the EQ-5D were replaced using multiple imputation. To impute the missing values on the five questions and the VAS score of the EQ-5D, five datasets were created $(m=5)$. The imputation model included the variables described in the conceptual model (Figure 4.1), plus the questions and VAS of the EQ-5D. Categorical variables were imputed as scale variables and variables containing more than two categories were converted into dummy variables. ${ }^{29,30}$ After multiple imputation, values for the separate questions of the EQ-5D were converted back to categorical variables. ${ }^{29}$

To obtain pooled estimates of the mean, variance and parametric tests the method as described by Rubin ${ }^{31}$ and more recently by Carlin et $\mathrm{al}^{32}$ was used. There is no generally accepted method for pooling the (adjusted) $R^{2}$, medians, quartiles and non-parametric tests. The (adjusted) $R^{2}$ was pooled by averaging the separate values for the (adjusted) $R^{2}$ from all 5 regression analyses. Medians and quartiles were pooled by obtaining the median of all 5 imputed datasets combined. Non-parametric tests were pooled by averaging the test statistic for the separate tests for all 5 imputed datasets, which was subsequently used to calculate $p$-values.

\section{Analyses}

First, descriptive statistics were computed for the population characteristics. Second, we examined differences in utility and VAS scores between subgroups with toxicity and without toxicity. For this purpose, patients were subdivided according to grade $0,1,2$ and $\geq 3$ for both xerostomia and dysphagia. Only subgroups with $\geq 10$ patients were included in the analyses. Subsequently, skewness and kurtosis of the HRQOL distribution within these subgroups were examined. If the skewness and kurtosis fell between -2.0 and 2.0, the differences between subgroups were compared using one way analysis of variance (ANOVA) and if significant multiple paired Student's t-tests were performed. Kruskal-Wallis tests and if statistically significant pairwise comparison MannWhitney $U$ tests were performed if the skewness or kurtosis exceeded this range.

Third, to test the conceptual model presented in Figure 4.1, utility and VAS scores were separately included as dependent variables in multivariate regression analyses. The variables which were considered to influence HRQOL were included in the regression model as independent variables. Grade of xerostomia and dysphagia, as well as sex, tumor location, surgery of the primary tumor and chemotherapy were included as categorical variables. Xerostomia and dysphagia were subdivided into dummy variables for grade 0 (reference category), 1, 2 and $\geq 3$. For tumor location dummy variables were created for oral cavity and lip, pharynx, larynx and other sites (reference category). Larynx and pharynx were separate categories since laryngeal carcinomas are typically early stage tumors and subsequently treated with limited radiation fields as opposed to pharynx 
carcinomas which are mostly locally advanced and treated with large radiation fields. Further, age and follow-up period after radiotherapy were included as scale variables. Since there is a possible interaction between xerostomia and dysphagia, effect modification (by means of an interaction term) was considered between these two variables.

Independently of the significance, none of the potentially influencing variables were excluded from the regression model. Only the interaction term between xerostomia and dysphagia was excluded if not statistically significant. The final regression models were checked for assumptions of linear regression.

All analyses were performed using SPSS 17.0. P-values $<0.05$ were considered statistically significant.

\section{Results}

\section{Population characteristics}

Altogether, $92 \%$ of all approached patients agreed to participate. In total 426 patients filled out the questionnaire, 30 of these patients were excluded since toxicity data were missing. This resulted in a study population of 396 patients of which 55 patients $(14 \%)$ had missing values on the EQ-5D which were imputed. Population characteristics are presented in Table 4.1. The average utility and VAS scores among all 396 patients were 0.85 (sd: 0.18) and 75 (sd: 15) respectively.

\section{HRQOL among patients subdivided by xerostomia and dysphagia}

Subgroups were categorized by grades of xerostomia and/or dysphagia (based on the RTOG scale). In total, 84 patients (21\%) had no xerostomia or dysphagia. Xerostomia was more prevalent than dysphagia (Figure 4.2).

For the utility scores, a ceiling effect (the scores are clustered around the upper end of the scale) was observed for the subgroup without toxicity. This resulted in a distribution with a high skewness (-2.4) and kurtosis (7.2). For VAS scores, the skewness and kurtosis were within the proposed range. Therefore, subgroup analyses were performed using non-parametric tests for utility scores and using parametric tests for VAS scores.

Utility scores were significantly different between subgroups (Kruskal-Wallis test, $\mathrm{P}<0.001$ ). Pairwise comparisons showed significantly higher utility scores for patients without toxicity compared to patients with $\geq$ grade 1 dysphagia independently of the grade of xerostomia. For patients with dysphagia grade 0 , average utility scores ranged between 0.91 (xerostomia grade 0 ) and 0.85 (xerostomia grade 2). For dysphagia grade 1 this ranged between 0.84 and 
0.82 and between 0.80 and 0.76 for $\geq$ grade 2 dysphagia (Table 4.2 and Figure 4.2).

Also VAS scores were significantly different among subgroups (ANOVA, $\mathrm{P}<$ 0.001). Pairwise comparisons showed significantly lower VAS scores if patients had $\geq$ grade 1 for both xerostomia and dysphagia, except for the subgroup with grade 1 xerostomia and grade 2 dysphagia. For patients with dysphagia grade 0 , average VAS scores ranged between 80 (xerostomia grade 0 ) and 76 (xerostomia grade 2). For dysphagia grade 1 this ranged between 74 and 70 and between 74 and 65 for $\geq$ grade 2 dysphagia (Table 4.2 and Figure 4.2).

Table 4.1: Characteristics of the study population*

\begin{tabular}{|c|c|c|c|c|c|}
\hline \multicolumn{3}{|l|}{ Patient characteristics } & \multicolumn{3}{|c|}{ Disease characteristics (continued) } \\
\hline Male & 276 & $70 \%$ & Node classification & & \\
\hline Female & 120 & $30 \%$ & No & 194 & $49 \%$ \\
\hline Age (years) & & & N1 & 57 & $14 \%$ \\
\hline Mean (Sd) & 63.2 & (11.8) & N2 & 121 & $31 \%$ \\
\hline Minimum, maximum & 20 & 99 & N3 & 9 & $2 \%$ \\
\hline Follow-up period (months) & & & Unknown & 15 & $4 \%$ \\
\hline Median (IR) & 20 & $(23.0)$ & Stage (UICC) & & \\
\hline \multirow[t]{2}{*}{ Minimum, maximum } & 6 & 152 & 0 & 7 & $2 \%$ \\
\hline & & & 1 & 55 & $14 \%$ \\
\hline Disease characteristics & & & $\|$ & 59 & $15 \%$ \\
\hline Tumor location & & & III & 78 & $20 \%$ \\
\hline Oral cavity and Lip & 51 & $13 \%$ & IV & 139 & $35 \%$ \\
\hline Pharynx & 115 & $29 \%$ & Unknown & 58 & $15 \%$ \\
\hline Larynx & 125 & $32 \%$ & & & \\
\hline Nasal cavity and sinuses & 20 & $5 \%$ & \multicolumn{3}{|l|}{ Treatment characteristics } \\
\hline Salivary gland & 23 & $6 \%$ & \multicolumn{3}{|l|}{ Surgery of the primary tumor } \\
\hline Other ${ }^{\dagger}$ & 49 & $12 \%$ & No & 258 & $65 \%$ \\
\hline Unknown & 13 & $3 \%$ & Yes & 138 & $35 \%$ \\
\hline Tumor classification & & & \multicolumn{3}{|l|}{ Chemotherapy } \\
\hline Tis & 8 & $2 \%$ & No & 339 & $86 \%$ \\
\hline T0 & 17 & $4 \%$ & Yes & 57 & $14 \%$ \\
\hline $\mathrm{T} 1$ & 92 & $23 \%$ & & & \\
\hline T2 & 115 & $29 \%$ & & & \\
\hline T3 & 55 & $14 \%$ & & & \\
\hline T4 & 82 & $21 \%$ & & & \\
\hline Unknown & 27 & $7 \%$ & & & \\
\hline
\end{tabular}

Abbreviations: $\mathrm{Sd}=$ standard deviation, $\mathrm{RT}=$ radiotherapy, $\mathrm{IR}=$ interquartile range

* Values are numbers (percentages) unless stated otherwise.

$\dagger$ Including skin and unknown primary tumors 


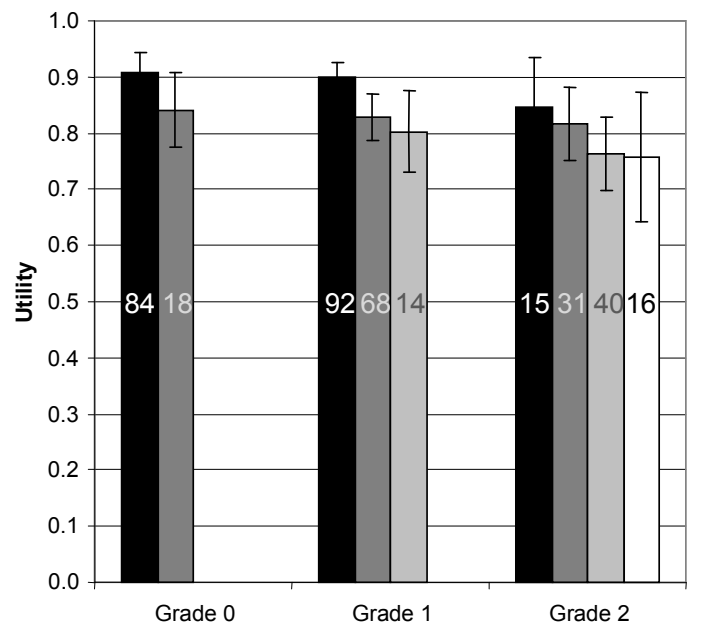

Xerostomia

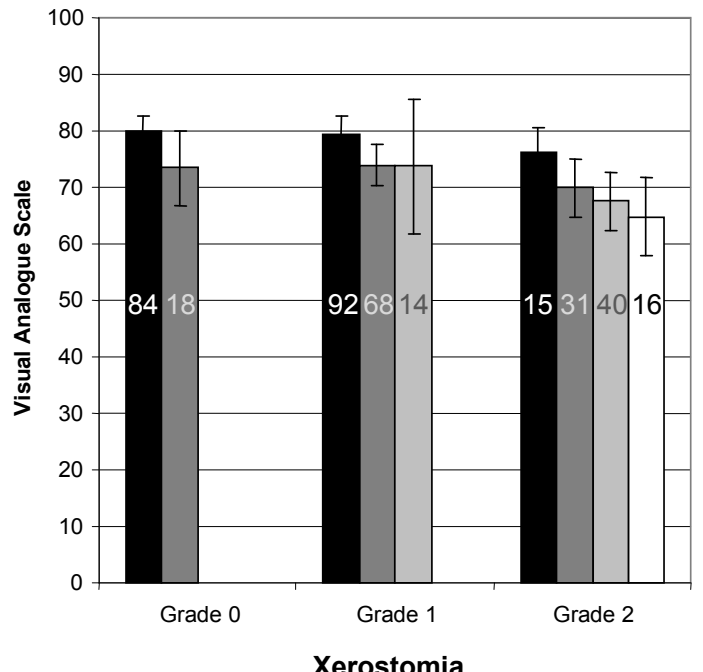

Dysphagia

- Grade 0

口Grade 1

$\square$ Grade 2

Grade 3+

Figure 4.2: HRQOL categorized by grade of xerostomia and dysphagia

Only subgroups with more than 10 patients are presented, error bars indicate the $95 \%$ confidence interval of the mean and values in the bars represent the $\mathrm{N}$ per subgroup. 


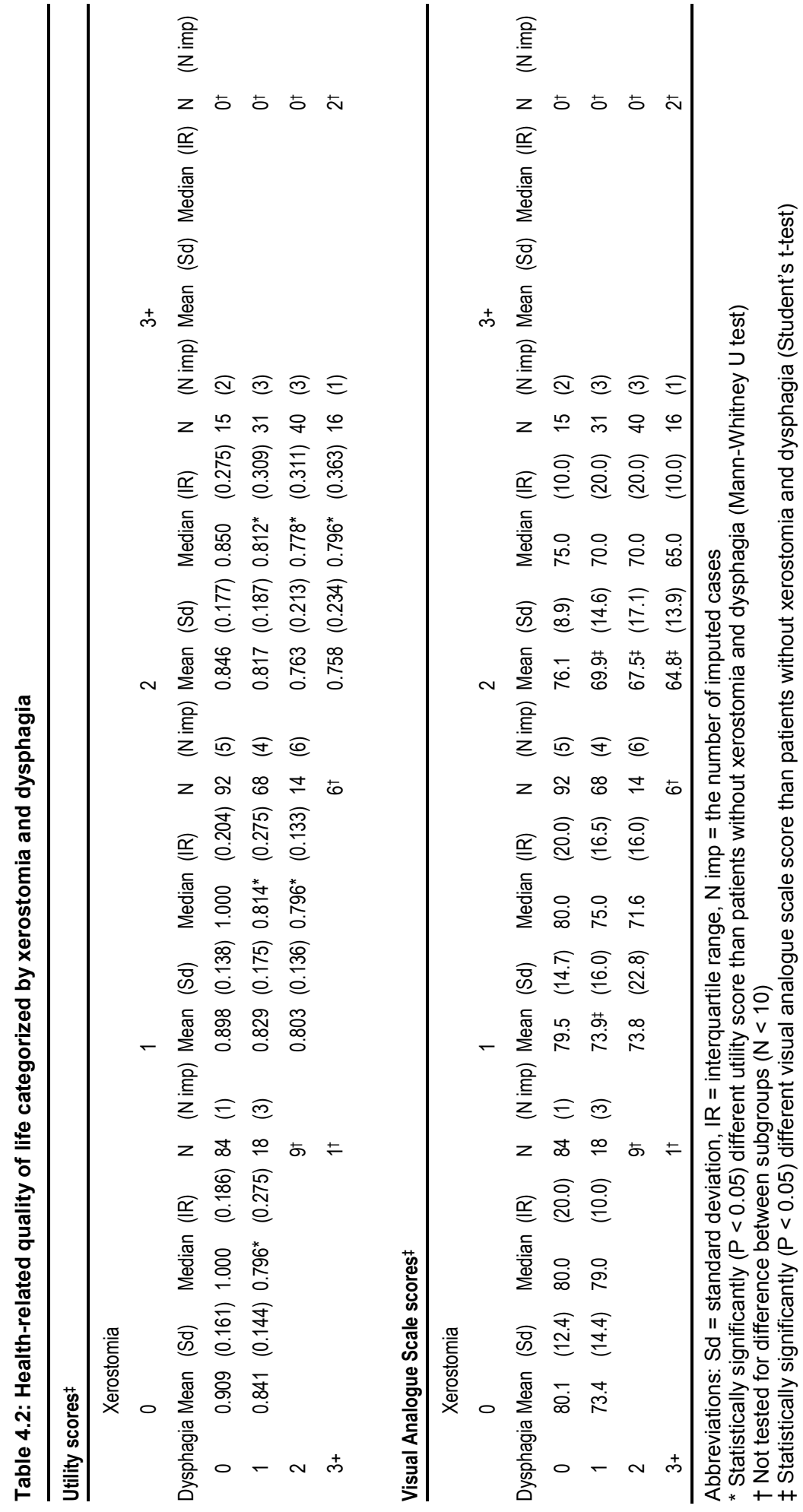


Table 4.3: Multivariate regression model ${ }^{*}$

\begin{tabular}{|c|c|c|c|c|c|c|}
\hline \multirow[b]{3}{*}{ Independent variables } & \multirow{2}{*}{\multicolumn{2}{|c|}{$\begin{array}{l}\text { Dependent variable: } \\
\text { utility score } \\
\mathrm{R}^{2}=42 \%^{\dagger} \\
\text { Adjusted } \mathrm{R}^{2}=17 \% \ddagger\end{array}$}} & & \multirow{2}{*}{\multicolumn{2}{|c|}{$\begin{array}{l}\text { Dependent variable: } \\
\text { VAS score } \\
R^{2}=39 \%^{\dagger}, \\
\text { Adjusted } R^{2}=16 \%^{\ddagger}\end{array}$}} & \multirow[b]{3}{*}{ P-value } \\
\hline & & & & & & \\
\hline & Beta & $\mathrm{Se}$ & P-value & Beta & Se & \\
\hline Constant & 0.836 & 0.057 & 0.000 & 79.4 & 4.9 & 0.000 \\
\hline \multicolumn{7}{|l|}{ Treatment toxicity } \\
\hline \multicolumn{7}{|l|}{ Xerostomiall } \\
\hline Grade 1 & -0.013 & 0.021 & 0.540 & -1.6 & 1.9 & 0.412 \\
\hline Grade 2 & -0.059 & 0.028 & 0.040 & -7.7 & 2.6 & 0.003 \\
\hline Grade 3+ & -0.336 & 0.123 & 0.006 & 8.2 & 11.1 & 0.460 \\
\hline \multicolumn{7}{|l|}{ Dysphagiall } \\
\hline Grade 1 & -0.059 & 0.021 & 0.005 & -5.6 & 1.8 & 0.002 \\
\hline Grade 2 & -0.126 & 0.029 & 0.000 & -7.2 & 3.0 & 0.026 \\
\hline Grade 3+ & -0.074 & 0.040 & 0.060 & -10.8 & 3.4 & 0.002 \\
\hline \multicolumn{7}{|l|}{ Patient characteristics } \\
\hline \multicolumn{7}{|l|}{ Sex } \\
\hline Male & 0.052 & 0.019 & 0.006 & 1.4 & 1.7 & 0.397 \\
\hline Age & 0.000 & 0.001 & 0.747 & -0.1 & 0.1 & 0.310 \\
\hline \multicolumn{7}{|l|}{ Disease characteristics } \\
\hline \multicolumn{7}{|l|}{ Tumor location\| } \\
\hline Oral cavity and lip & 0.081 & 0.030 & 0.006 & 5.9 & 2.7 & 0.031 \\
\hline Pharynx & 0.063 & 0.027 & 0.018 & 4.7 & 2.3 & 0.040 \\
\hline Larynx & 0.032 & 0.026 & 0.226 & 1.9 & 2.2 & 0.398 \\
\hline \multicolumn{7}{|l|}{ Treatment characteristics } \\
\hline \multicolumn{7}{|l|}{ Surgery of the primary tumor } \\
\hline Yes & 0.062 & 0.022 & 0.006 & 4.0 & 2.0 & 0.043 \\
\hline \multicolumn{7}{|l|}{ Chemotherapy } \\
\hline Yes & 0.040 & 0.027 & 0.148 & 4.4 & 2.4 & 0.068 \\
\hline Follow-up period & 0.000 & 0.000 & 0.499 & 0.0 & 0.0 & 0.757 \\
\hline \multicolumn{7}{|l|}{ Interaction } \\
\hline Xerostomia and Dysphagia & - & - & ns & - & - & ns \\
\hline
\end{tabular}

Abbreviations: $\mathrm{VAS}=$ Visual Analogue Scale, $\mathrm{Se}=$ standard error, $\mathrm{ns}=$ not statistically significant ${ }^{*}$ For the complete case analyses see Appendix 4.2

$\dagger R^{2}=$ the fraction of the total squared error that is explained by the regression model. ${ }^{33}$

$\ddagger$ Adjusted $R^{2}=$ measure indicating how much variance in the outcome would be accounted for if the model had been derived from the population from which the sample was taken. ${ }^{33}$

\| Reference category for dummy variables was Grade 0 toxicity and other tumor locations.

\section{Factors influencing HRQOL}

Utility scores were significantly negatively affected by grade 2 and $\geq 3$ xerostomia in the multivariate regression analyses (Table 4.3). This was also true for dysphagia grade 1 and grade 2 . Grade 1 xerostomia and $\geq$ grade 3 dysphagia had no significant impact on utility scores. Other variables that have a significant influence on utility scores were sex, tumor location and whether surgery of the primary tumor was performed. The interaction term for xerostomia and 
dysphagia was not significant and therefore excluded from the regression model.

VAS scores were significantly negatively influenced by grade 2 xerostomia in the multivariate regression analyses (Table 4.3). Also, all grades of dysphagia (grade 1, 2 and $\geq 3$ ) had a significantly negative impact on VAS scores. Xerostomia grade 1 and $\geq 3$ had no significant impact on VAS scores. Other statistically significant variables in the regression model were: tumor location, whether surgery of the primary tumor was performed and whether patients received chemotherapy. The interaction term for xerostomia and dysphagia was not statistically significant and therefore excluded from the regression model.

The regression models were checked for the assumptions of linear regression and no severe deficiencies were observed.

\section{Discussion}

To the best of our knowledge, this study was the first to analyse the impact of late treatment-related toxicity on patient-rated utility and VAS scores in HNC. Our analyses demonstrated that dysphagia and xerostomia both have a negative impact on HRQOL. Moreover, dysphagia had the largest impact on HRQOL. Except for age, follow-up period and whether patients received chemotherapy, all independent variables reported in the conceptual model (Figure 4.1) were significant predictors of $\mathrm{HRQOL}$ in at least one of the two regression analyses. The relations were as expected, except for surgery. The complete case analyses resulted in comparable regression models (Appendix 4.2).

Potential limitations of this study included the low number of patients with grade $\geq 3$ toxicity (Table 4.2 ). This might explain the fact that grade $\geq 3$ dysphagia was not a significant predictor of utility scores. The same may apply to grade $\geq 3$ xerostomia for VAS scores. Also, the cross-sectional design and hence the inability to correct for baseline HRQOL may be considered as a limitation. However, we corrected for potentially confounding variables and to our knowledge there was no rationale to assume that the HRQOL before treatment was on average different between the subgroups subdivided by treatment toxicity. Therefore, the impact of late treatment-related toxicity on HRQOL in our study can probably not be attributed to the cross-sectional design. Nevertheless, prospective studies measuring utility and VAS scores in HNC patients with different grades of toxicity are needed to confirm our results. Finally, we included patients who had a follow-up period of at least six months from treatment start. This includes patients who have been studied 4-5 months following completion of radiotherapy. It is unclear whether xerostomia and dysphagia have stabilized after this period. However, additional analyses showed that if the 21 patients $(5 \%)$ with a shorter follow-up than six months from treatment comple- 
tion were excluded, the HRQOL estimates stratified by xerostomia and dysphagia remained similar.

The current study showed on average relatively higher utility than VAS scores. In contrast with our results, previous studies indicated that patients with a specific condition (patients' perspective) are inclined to place higher scores for their own health state compared with non-patients (general public perspective), presumably due to adaptation. ${ }^{34-37}$ However, corresponding with our results, most studies indicated that due to differences in measurement methods, utility scores based on the TTO method (as in this study) are higher than VAS scores. ${ }^{6,34}$ Despite these abovementioned differences between utility and VAS scores, the decrements between the subgroups subdivided by xerostomia and dysphagia were comparable between the two methods. Therefore, the differences between utility and VAS scores probably represent an overall shift in HRQOL for all subgroups and do not alter the estimated impact of xerostomia and dysphagia on HRQOL.

Previously, utility scores in HNC have been estimated using various methods. ${ }^{7-}$ ${ }^{11}$ Based on the EQ-5D filled out by 50 oncology nurses, Brown et al reported an average utility score of 0.86 for post-treatment HNC patients with locoregional disease control. ${ }^{7}$ This corresponds with the overall average utility score of 0.85 in our study. Ringash et al applied the TTO method in 112 disease free laryngeal cancer patients resulting in a higher utility score of $0.91{ }^{10}$ Due to difference in patient populations or subgroups it was not possible to compare utility scores from the other studies with our results. ${ }^{8,9,11}$

Marra et al, ${ }^{38}$ defined 0.03 as a minimally important difference in utility scores (measured by the EQ-5D). Accordingly, the main clinical implications of our results are that treatment strategies aimed at reducing dysphagia and/or xerostomia have the potential to result in a meaningful improvement of generic HRQOL. This emphasizes that next to the expected length of survival also treatment toxicity should be considered when treatment choices are made. Our results can be used to inform this trade-off. In consideration of this trade-off, xerostomia is more prevalent than dysphagia, whereas dysphagia has a higher impact on HRQOL than xerostomia. Therefore, preventing xerostomia could benefit more patients, whereas preventing dysphagia might result in a larger benefit per patient.

To reduce treatment toxicity, it may be useful to focus on patients who have the highest risk of experiencing xerostomia and/or dysphagia. In our study population, 84 patients $(21 \%)$ had no toxicity. Compared with the 312 patients $(79 \%)$ with any grade of toxicity, the patient group without toxicity consisted of less patients with advanced disease stage (III/IV: $27 \%$ versus $62 \%$ ), had relatively less pharyngeal cancer patients (29\% versus $43 \%)$ and more laryngeal cancer patients (36\% versus $5 \%$ ). Hence, patients with advanced disease stage and/or pharyngeal cancer may have a higher chance of experiencing xerostomia and/or dysphagia. However, this needs to be confirmed in prospective studies. 
In conclusion, our results can be used to support clinical decision-making. They underscore the importance that, next to survival data, clinical studies examine toxicity and its impact on generic HRQOL. This assists the trade-off between length and quality of survival. Our study suggests that xerostomia and dysphagia have a negative impact on HRQOL; moreover it was found that dysphagia affects patients' $H R Q O L$ stronger than xerostomia.

\section{Acknowledgement}

Ann Claessens, Ria Debougnoux, Prof. dr. Bernd Kremer, Dr. Kenneth Kross, Dr. Martin Lacko, Chantal Overhof and Michel Walthouwer are gratefully acknowledged for their support during the data collection. 


\section{References}

1. Langendijk JA, Doornaert P, Verdonck-de Leeuw IM, et al: Impact of late treatment-related toxicity on quality of life among patients with head and neck cancer treated with radiotherapy. $J$ Clin Oncol 26:3770-6, 2008

2. Hammerlid E, Taft C: Health-related quality of life in long-term head and neck cancer survivors: a comparison with general population norms. Br J Cancer 84:149-56, 2001

3. Rogers SN, Ahad SA, Murphy AP: A structured review and theme analysis of papers published on 'quality of life' in head and neck cancer: 2000-2005. Oral Oncol 43:843-68, 2007

4. Sayed SI, Elmiyeh B, Rhys-Evans P, et al: Quality of life and outcomes research in head and neck cancer: A review of the state of the discipline and likely future directions. Cancer Treat Rev, 2009

5. Bentzen SM, Dorr W, Anscher MS, et al: Normal tissue effects: reporting and analysis. Semin Radiat Oncol 13:189-202, 2003

6. Drummond MF, Sculpher MJ, Torrance GW, et al: Methods for the Economic Evaluation of Health Care Programmes. Oxford Oxford University Press 2005

7. Brown B, Diamantopoulos A, Bernier J, et al: An economic evaluation of cetuximab combined with radiotherapy for patients with locally advanced head and neck cancer in Belgium, France, Italy, Switzerland, and the United Kingdom. Value Health 11:791-9, 2008

8. Paleri V, Kelly CG: Re-irradiation with concurrent chemotherapy in recurrent head and neck cancer: a decision analysis model based on a systematic review. Clin Otolaryngol 33:331-7, 2008

9. Schneider SM, Pouget I, Staccini P, et al: Quality of life in long-term home enteral nutrition patients. Clin Nutr 19:23-8, 2000

10. Ringash J, Redelmeier DA, O'Sullivan B, et al: Quality of life and utility in irradiated laryngeal cancer patients. Int J Radiat Oncol Biol Phys 47:875-81, 2000

11. Schwartz SR, McDowell J, Yueh B: Numeracy and the shortcomings of utility assessment in head and neck cancer patients. Head Neck 26:401-7, 2004

12. Lovell SJ, Wong HB, Loh KS, et al: Impact of dysphagia on quality-of-life in nasopharyngeal carcinoma. Head Neck 27:864-72, 2005

13. Fryback DG, Dunham NC, Palta M, et al: US norms for six generic health-related quality-of-life indexes from the National Health Measurement study. Med Care 45:1162-70, 2007

14. Hanmer J, Hays RD, Fryback DG: Mode of administration is important in US national estimates of health-related quality of life. Med Care 45:1171-9, 2007

15. Kind $P$, Hardman G, Macran S: UK population norms for EQ-5D Accessed on http://ideas.repec.org/p/chy/respap/172chedp.html

16. Terrell JE, Ronis DL, Fowler KE, et al: Clinical predictors of quality of life in patients with head and neck cancer. Arch Otolaryngol Head Neck Surg 130:401-8, 2004

17. Fang FM, Tsai WL, Chien CY, et al: Changing quality of life in patients with advanced head and neck cancer after primary radiotherapy or chemoradiation. Oncology 68:405-13, 2005

18. Allison PJ, Locker D, Wood-Dauphinee $S$, et al: Correlates of health-related quality of life in upper aerodigestive tract cancer patients. Qual Life Res 7:713-22, 1998

19. Jellema AP, Slotman BJ, Doornaert $P$, et al: Impact of radiation-induced xerostomia on quality of life after primary radiotherapy among patients with head and neck cancer. Int $\mathrm{J}$ Radiat Oncol Biol Phys 69:751-60, 2007

20. ClinicalTrials.gov: Quality of Life in Patients Treated for Head and Neck Cancer (NCT00963586) Accessed on 18-05-2010. http://clinicaltrials.gov/ct2/show/NCT00963586

21. EuroQol Group: EuroQol--a new facility for the measurement of health-related quality of life. The EuroQol Group. Health Policy 16:199-208, 1990

22. Cox JD, Stetz J, Pajak TF: Toxicity criteria of the Radiation Therapy Oncology Group (RTOG) and the European Organization for Research and Treatment of Cancer (EORTC). Int J Radiat Oncol Biol Phys 31:1341-6, 1995 
23. Brauer $C A$, Rosen $A B$, Greenberg $D$, et al: Trends in the measurement of health utilities in published cost-utility analyses. Value Health 9:213-8, 2006

24. Rasanen $\mathrm{P}$, Roine $\mathrm{E}$, Sintonen $\mathrm{H}$, et al: Use of quality-adjusted life years for the estimation of effectiveness of health care: A systematic literature review. Int J Technol Assess Health Care 22:235-41, 2006

25. National Institute for Health and Clinical Excellence: Guide to the methods of technology appraisal Accessed on http://www.nice.org.uk/media/B52/A7/TAMethodsGuideUpdatedJune2008.pdf

26. Dolan P: Modeling valuations for EuroQol health states. Med Care 35:1095-108, 1997

27. Cheung KY, Oemar M, Oppe M, et al: User Guide: Basic information on how to use EQ-5D Accessed http://www.euroqol.org/fileadmin/user_upload/Documenten/PDF/Office/User_Guide_v3_April_2 010_.pdf

28. Fielding $S$, Fayers $P$, Ramsay $C$ : Predicting missing quality of life data that were later recovered: an empirical comparison of approaches. Clin Trials 7:333-42, 2010

29. Allison PD: Missing Data. Thousand Oaks, Sage, 2002

30. Graham JW: Missing data analysis: making it work in the real world. Annu Rev Psychol 60:54976, 2009

31. Rubin DB: Multiple imputation for nonresponse in surveys. New York, John Wiley, 1987

32. Carlin JB, Li N, Greenwood P, et al: Tools for analyzing multiple imputed datasets. Stata Journal 3:226-244, 2003

33. Field A: Discovering Statistics Using SPSS (ed 2nd edition), Sage Publications Ltd, 2005

34. Brauer CA, Neumann PJ: Quality-adjusted life years: how useful in medico economic studies. Fundam Clin Pharmacol 19:603-7, 2005

35. Dolan P: Valuing health-related quality of life. Issues and controversies. Pharmacoeconomics 15:119-27, 1999

36. Dolan P: Whose preferences count? Med Decis Making 19:482-6, 1999

37. Rashidi AA, Anis AH, Marra CA: Do visual analogue scale (VAS) derived standard gamble (SG) utilities agree with Health Utilities Index utilities? A comparison of patient and community preferences for health status in rheumatoid arthritis patients. Health Qual Life Outcomes 4:25, 2006

38. Marra CA, Woolcott JC, Kopec JA, et al: A comparison of generic, indirect utility measures (the HUI2, HUI3, SF-6D, and the EQ-5D) and disease-specific instruments (the RAQoL and the $\mathrm{HAQ}$ ) in rheumatoid arthritis. Soc Sci Med 60:1571-82, 2005 


\begin{tabular}{|c|c|c|}
\hline & Organ tissue & \\
\hline & Salivary Glands & Esophagus \\
\hline Grade 0 & None & None \\
\hline Grade 1 & $\begin{array}{l}\text { Slight dryness of mouth } \\
\text { Good response on stimulation }\end{array}$ & $\begin{array}{l}\text { Mild fibrosis } \\
\text { Slight difficulty in swallowing solids } \\
\text { No pain on swallowing }\end{array}$ \\
\hline Grade 2 & $\begin{array}{l}\text { Moderate dryness of mouth } \\
\text { Poor response on stimulation }\end{array}$ & $\begin{array}{l}\text { Unable to take solid food normally } \\
\text { Swallowing semi-solid food } \\
\text { Dilatation may be indicated }\end{array}$ \\
\hline Grade 3 & $\begin{array}{l}\text { Complete dryness of mouth } \\
\text { No response on stimulation }\end{array}$ & $\begin{array}{l}\text { Severe fibrosis } \\
\text { Able to swallow only liquids } \\
\text { May have pain on swallowing } \\
\text { Dilation required }\end{array}$ \\
\hline Grade 4 & Fibrosis & $\begin{array}{l}\text { Necrosis/ Perforation } \\
\text { Fistula }\end{array}$ \\
\hline Grade 5 & Death directly related to late radiation effects & Death directly related to late radiation effects \\
\hline
\end{tabular}




\section{Appendix 4.2: Complete case analyses of the multivariate regression} model

\begin{tabular}{|c|c|c|c|c|c|c|}
\hline \multirow[b]{3}{*}{ Independent variables } & \multirow{2}{*}{\multicolumn{3}{|c|}{$\begin{array}{l}\text { Dependent variable: } \\
\text { utility score } \\
\mathrm{R}^{2}=43 \%^{\dagger}, \\
\text { Adjusted } \mathrm{R}^{2}=18 \%^{\ddagger}\end{array}$}} & \multirow{2}{*}{\multicolumn{3}{|c|}{$\begin{array}{l}\text { Dependent variable: } \\
\text { VAS score } \\
\mathrm{R}^{2}=38 \%^{\dagger}, \\
\text { Adjusted } \mathrm{R}^{2}=14 \%^{\ddagger}\end{array}$}} \\
\hline & & & & & & \\
\hline & Beta & Se & P-value & Beta & Se & P-value \\
\hline Constant & 0.836 & 0.059 & 0.000 & 80.6 & 5.1 & 0.000 \\
\hline \multicolumn{7}{|l|}{ Treatment toxicity } \\
\hline \multicolumn{7}{|l|}{ Xerostomiall } \\
\hline Grade 1 & -0.014 & 0.022 & 0.529 & -1.9 & 1.9 & 0.308 \\
\hline Grade 2 & -0.069 & 0.030 & 0.020 & -7.6 & 2.5 & 0.003 \\
\hline Grade 3+ & -0.346 & 0.125 & 0.006 & -6.0 & 14.8 & 0.684 \\
\hline \multicolumn{7}{|l|}{ Dysphagiall } \\
\hline Grade 1 & -0.057 & 0.022 & 0.009 & -5.6 & 1.9 & 0.003 \\
\hline Grade 2 & -0.128 & 0.031 & 0.000 & -7.2 & 2.7 & 0.007 \\
\hline Grade 3+ & -0.071 & 0.041 & 0.083 & -9.7 & 3.7 & 0.008 \\
\hline \multicolumn{7}{|l|}{ Patient characteristics } \\
\hline \multicolumn{7}{|l|}{ Sex } \\
\hline Male & 0.051 & 0.020 & 0.009 & 1.1 & 1.7 & 0.528 \\
\hline Age & 0.000 & 0.001 & 0.807 & -0.1 & 0.1 & 0.215 \\
\hline \multicolumn{7}{|l|}{ Disease characteristics } \\
\hline \multicolumn{7}{|l|}{ Tumor location\| } \\
\hline Oral cavity and lip & 0.079 & 0.031 & 0.010 & 5.0 & 2.7 & 0.064 \\
\hline Pharynx & 0.065 & 0.027 & 0.019 & 4.5 & 2.4 & 0.062 \\
\hline Larynx & 0.031 & 0.027 & 0.249 & 1.9 & 2.4 & 0.430 \\
\hline \multicolumn{7}{|l|}{ Treatment characteristics } \\
\hline \multicolumn{7}{|l|}{ Surgery of the primary tumor } \\
\hline Yes & 0.066 & 0.023 & 0.005 & 4.1 & 2.0 & 0.043 \\
\hline \multicolumn{7}{|l|}{ Chemotherapy } \\
\hline Yes & 0.043 & 0.028 & 0.125 & 4.0 & 2.5 & 0.103 \\
\hline Follow-up period & 0.000 & 0.000 & 0.451 & 0.0 & 0.0 & 0.436 \\
\hline \multicolumn{7}{|l|}{ Interaction } \\
\hline Xerostomia and Dysphagia & - & - & ns & - & - & ns \\
\hline
\end{tabular}

* Interaction between xerostomia and dysphagia was not statistically significant $\dagger \mathrm{R} 2$ = the fraction of the total squared error that is explained by the regression model.

¥ Adjusted R2 = measure indicating how much variance in the outcome would be accounted for if the model had been derived from the population from which the sample was taken.

|| Reference category for dummy variables was Grade 0 toxicity and other tumor locations. 


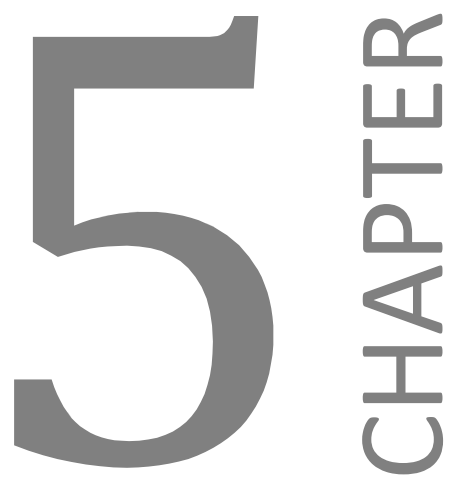

\title{
PROTONS IN HEAD AND NECK CANCER: BRIDGING THE GAP OF EVIDENCE
}

\author{
Bram Ramaekers \\ Janneke Grutters \\ Madelon Pijls-Johannesma \\ Philippe Lambin \\ Manuela Joore \\ Johannes Langendijk
}

International Journal of Radiation Oncology*Biology*Physics. 2013

Apr;85(5):1282-8 


\section{Abstract}

Objective: To explore the (cost-)effectiveness of swallowing sparing intensitymodulated proton radiotherapy (IMPT) compared with swallowing sparing intensity-modulated radiotherapy with photons (IMRT) in head and neck cancer (HNC).

Methods: A Markov model was constructed to examine and compare the costs and quality-adjusted life years (QALYs) of the following strategies: 1) IMPT for all patients; 2) IMRT for all patients and; 3) IMPT if efficient. The assumption of equal survival for IMPT and IMRT in the base case analysis was relaxed in a sensitivity analysis.

Results: IMPT and IMRT for all patients yielded 6.620 and 6.520 QALYs and costed $€ 50,989$ and $€ 41,038$, respectively. IMPT if efficient yielded 6.563 QALYs and costed $€ 43,650$. The incremental cost-effectiveness ratio of IMPT if efficient versus IMRT for all patients was $€ 60,278$ per QALY gained.

In the sensitivity analysis, IMRT was more effective (0.967 QALYs) and less expensive $(€ 8,218)$ and thus dominated IMPT for all patients.

Conclusions: Cost-effectiveness analysis based on NTCP models and planning studies proved feasible and informative and enables the analysis of individualized strategies. The increased effectiveness of IMPT does not seem to outweigh the higher costs for all HNC patients. However, when assuming equal survival among both modalities, there seems value in identifying those patients for whom IMPT is cost-effective.

Keywords: Cost-effectiveness; Proton radiotherapy; Intensity-modulated radiotherapy; Head and neck cancer; Quality-adjusted life years; Markov model 


\section{Introduction}

The costs of cancer care are expected to accelerate due to the ageing population and costly new treatments such as proton radiotherapy. ${ }^{1,2}$ Since resources are scarce, it is important to consider the (cost-)effectiveness of new technologies. ${ }^{2}$ Economic evaluations are often performed using decision-analytic modeling to examine the cost-effectiveness ratio and guide evidence-based decisionmaking under uncertainty. ${ }^{3}$ Economic evaluations frequently rely on comparative effectiveness research to estimate the effectiveness, patient reported outcomes and resource use. However, comparative effectiveness research is sparsely available for proton radiotherapy. ${ }^{4}$ Normal tissue complication probability (NTCP) models combined with comparative planning studies might be informative to bridge this gap of evidence. NTCP models estimate the probability of toxicity based on the expected radiation dose to healthy tissues. Comparative planning studies compare the dose-distributions in patients for different radiotherapy techniques. Hence, NTCP models and comparative planning studies can be used in economic evaluations to estimate the expected benefit of innovative radiotherapy techniques. To explore this methodology, we examine the cost-effectiveness of intensity modulated proton radiotherapy (IMPT) opposed to the current standard: intensity-modulated radiotherapy with photons (IMRT) in head and neck cancer (HNC).

After radiotherapy for $\mathrm{HNC}$, treatment-related toxicities like xerostomia and dysphagia substantially affect patients' health-related quality of life. ${ }^{5}$ Planning studies suggest that proton radiotherapy, with its favorable in-depth dose distribution, has the ability to reduce the radiation dose to healthy tissues and hence the occurrence of toxicity compared with photons. ${ }^{6}$ However, there is no clinical evidence which supports these theoretical benefits of protons. ${ }^{4,6}$ Therefore, we aimed to combine NTCP models and comparative planning data in a modelbased economic evaluation to explore the (cost-)effectiveness of swallowing sparing IMPT (scanned) compared with swallowing sparing IMRT for HNC patients. Swallowing sparing techniques have the ability to reduce the dose to swallowing structures with similar dose to the parotid and submandibular glands compared with standard techniques. Consequently, swallowing sparing techniques may reduce the occurrence of dysphagia and hence limit the impact of treatment on quality of life. ${ }^{5,7}$ These swallowing sparing techniques can be considered the best available IMRT and IMPT treatments. It is expected that not all HNC patients have an equal expected benefit from IMPT. Therefore, we will also examine an individualized strategy wherein IMPT is only administered to patients for whom IMPT is expected to be cost-effective. 


\section{Methods}

\section{Markov model description}

The study population consisted of locally advanced (stage III-IV) HNC patients (oral cavity, laryngeal and pharyngeal cancer), aged on average 61 years at start radiotherapy and pre-treatment RTOG <grade 2 dysphagia and xerostomia. A decision-analytic Markov cohort model was constructed to estimate the expected costs and effects of three treatment strategies:

1. IMPT for all patients;

2. IMRT for all patients;

3. IMPT if efficient: patients for whom IMPT is expected to be cost-effective receive IMPT, the remaining patients receive IMRT.

Our analysis focuses on the question what type of radiotherapy should be provided if radiotherapy is the therapy of choice. Since surgery is complementary to radiotherapy, it is not considered as comparator.

Through transiting a hypothetical cohort of patients between mutually exclusive health states, a Markov model aims to reflect the course of a disease to compare outcomes for competing interventions. ${ }^{3}$ The Markov model consisted of seven health states (Figure 5.1):

(a) Disease free without toxicity

(b) Disease free with xerostomia RTOG $\geq$ grade 2

(c) Disease free with xerostomia and dysphagia RTOG $\geq$ grade 2

(d) Disease free with dysphagia RTOG $\geq$ grade 2

(e) Loco-regional recurrence

(f) Distant metastasis

(g) Death

To incorporate the reversibility of acute toxicity during the first six months after radiotherapy, a cycle time of six months was used in the first year; afterwards the cycle time was one year. A lifetime time horizon was used.

\section{Markov model assumptions}

The main assumption was that disease progression (including radiation-induced cancer) and thus survival were equal for the comparators. This was assumed since the tumor dose in the planning studies used to estimate toxicity was similar for both modalities and available clinical evidence does not show statistically significant differences in survival. ${ }^{6}$ Secondly, toxicity occurring in the first 6 months was (partly) acute toxicity and thus (partly) reversible. Patients can for instance transit from disease free with xerostomia to disease free without toxicity after the first six months. Thereafter, toxicity was assumed to be irreversible. 


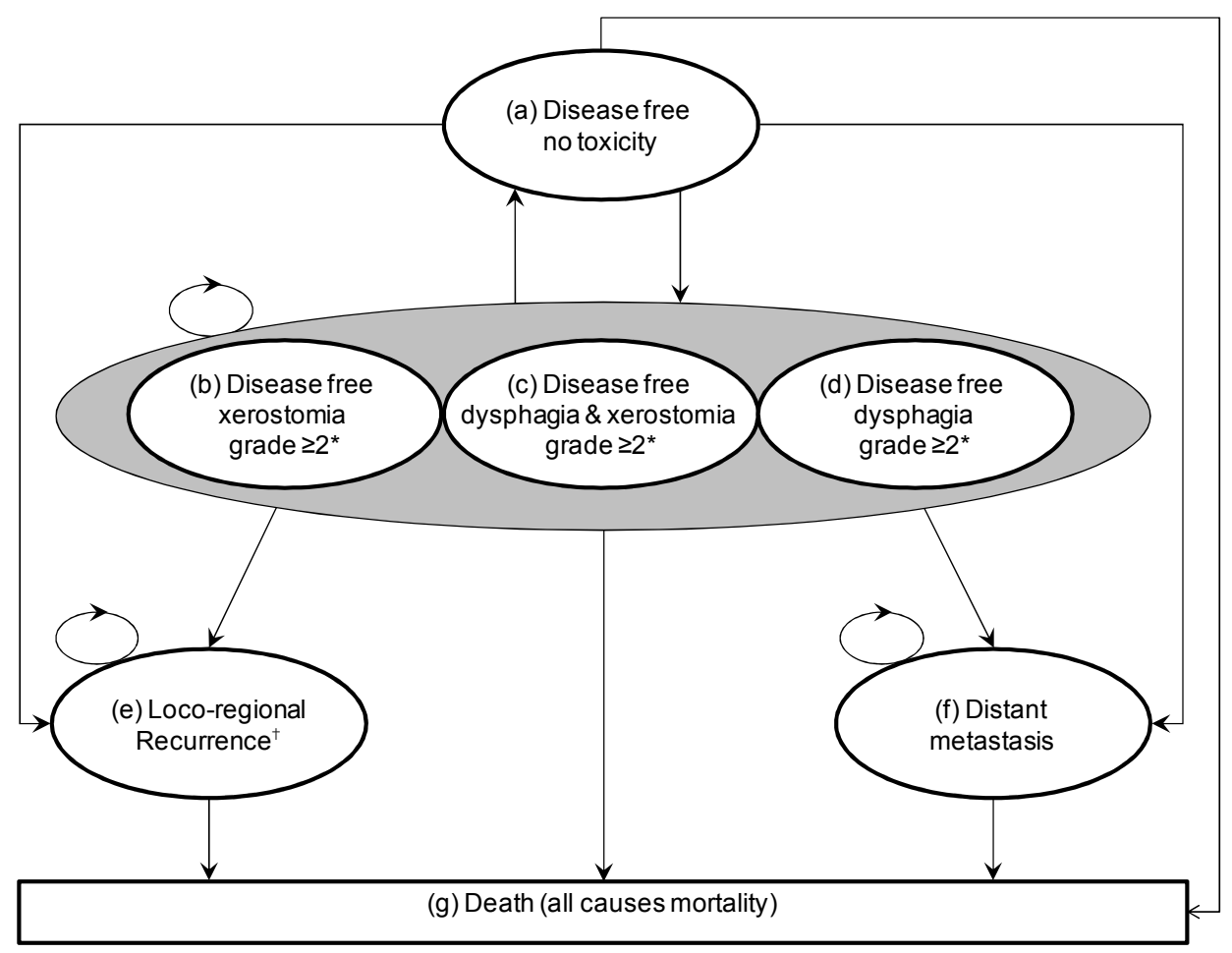

Figure 5.1: Diagrammatical representation of the model structure

Toxicity that occurred in the first 6 months was (partly) reversible. Therefore, patients are allowed to move between health state $a, b, c$ and $d$ six months after radiotherapy. Thereafter, toxicity was assumed to be irreversible.

* Toxicity was defined according to the presence of RTOG grade 2 or higher

† There was no transition from loco-regional recurrence to death via distant metastasis. This was done firstly because patients who develop distant metastasis are expected to die within one year (Appendix 5.3). If we would add the transition from loco-regional recurrence to death via distant metastasis, it will last one year or more before patients die due to distant metastasis. Secondly, the intermediate step to distant metastasis was already included in the probabilities used to calculate death after loco-regional recurrence (Appendix 5.3).

\section{Markov model input}

\section{Transition probabilities}

The occurrence of xerostomia and/or dysphagia was estimated based on two available NTCP models. ${ }^{8,9}$ Mean radiation dose to the parotis ipsilateral and parotis contralateral were used to predict xerostomia RTOG $\geq$ grade 2 at 6 and 12 months after radiotherapy. ${ }^{8}$ Mean dose to the pharyngeal constrictor muscle superior and the supraglottic area predicted dysphagia RTOG $\geq$ grade 2 at 6 and 12 months after radiotherapy (Appendix 5.1). ${ }^{9}$ The required dose parameters were retrieved from a planning study $(\mathrm{N}=25)$ comparing swallowing sparing IMRT and swallowing sparing IMPT. ${ }^{7}$ Subsequently, the individual dose param- 
eters and NTCP models were used to calculate individual IMPT/IMRT toxicity probabilities. These individual probabilities were averaged to obtain the average probabilities. The toxicity probabilities for IMPT if efficient were obtained by firstly determining which treatment (IMPT/IMRT) was expected to be costeffective for each individual patient. This was done by using the individual dose parameters and toxicity probabilities to calculate individual cost-effectiveness. Secondly, in patients for whom IMPT was expected to be cost-effective, the IMPT probabilities were used, while the IMRT probabilities were used in the remaining patients. Thirdly, the obtained individual probabilities were averaged (calculation is illustrated in Table 5.1).

The proportion of patients who had both xerostomia and dysphagia was calculated using conditional toxicity probabilities from a cross-sectional survey (Appendices 5.1 and 5.2$)^{5}$

Table 5.1: Method to calculate toxicity for the IMPT if efficient strategy: illustrated for xerostomia 6 months after radiotherapy

\begin{tabular}{|c|c|c|c|c|c|}
\hline \multirow[b]{2}{*}{ Patient } & \multicolumn{2}{|c|}{ Probability of xerostomia } & \multirow[t]{2}{*}{ ICER } & \multirow{2}{*}{$\begin{array}{l}\text { Preferred } \\
\text { IMPT/IMRT }\end{array}$} & \multirow{2}{*}{$\begin{array}{l}\text { Probability } \\
\text { xerostomia } \\
\text { IMPT if efficient }\end{array}$} \\
\hline & IMPT & IMRT & & & \\
\hline 1 & $25.5 \%$ & $41.3 \%$ & $€ 93,302$ & IMRT & $41.3 \%$ \\
\hline 2 & $18.9 \%$ & $36.6 \%$ & $€ 169,448$ & IMRT & $36.6 \%$ \\
\hline 3 & $23.6 \%$ & $55.2 \%$ & $€ 44,358$ & IMPT & $23.6 \%$ \\
\hline 4 & $26.7 \%$ & $37.2 \%$ & $€ 150,041$ & IMRT & $37.2 \%$ \\
\hline 25 & $25.8 \%$ & $45.1 \%$ & $€ 89,593$ & IMRT & $45.1 \%$ \\
\hline$\overline{\text { Mean pr }}$ & ability & a for the & ent strateg & & $37.1 \%$ \\
\hline
\end{tabular}

Abbreviations: ICER = Incremental cost-effectiveness ratio, vs = versus

*Patients will only receive IMPT in this scenario if IMPT is expected to be cost-effective compared with IMRT (grey fields), thus if the ICER is below the threshold of $€ 80,000$ per QALY gained.

Disease progression for all comparators was based on a meta-analysis which compared radiotherapy with and without chemotherapy in curatively treated non-metastatic $\mathrm{HNC}^{10}{ }^{10}$ These probabilities were extracted from the concomitant chemotherapy arm (current standard treatment for advanced HNC). Age dependent background mortality was used for disease free patients. An increased mortality probability was used for patients who had loco-regional recurrence or distant metastases (Appendix 5.3). 


\section{Effects and costs}

Quality of life in terms of utility scores was used as outcome measure. Utility scores provide a single index value for health status ranging from 0 (death) to 1 (full health). Utility scores were derived from a cross-sectional study $(\mathrm{N}=396)$ using the Euroqol-5D questionnaire in Dutch HNC patients (Appendix 5.3). ${ }^{5}$ Utility scores were combined with life expectancy to calculate quality adjusted life years (QALYs).

The health care perspective was used to calculate costs using activity based costing. Unit prices and resource use were based on guidelines, a crosssectional survey ${ }^{5}$ or if necessary expert opinion (Appendices 5.4 and 5.5).

The primary treatment costs for IMPT were calculated by multiplying treatment costs for IMRT with a cost ratio of 2.1. ${ }^{1,11}$ For IMPT if efficient, both IMPT and IMRT treatment plans were made to compare individual dose distributions and decide upon the most efficient treatment per patient. Therefore, costs of an extra treatment plan (€88) were added for this strategy.

A half-cycle correction was applied for QALYs and costs. ${ }^{12}$ Future QALYs and costs were discounted by rates of $1.5 \%$ and $4.0 \%$ respectively. ${ }^{13}$ All costs were converted to the 2010 price level.

\section{Markov model analyses}

Expected mean costs, occurrence of toxicity, disease and toxicity free life years (DTFLYs) and QALYs were estimated for all comparators. Subsequently, the incremental cost-effectiveness ratio (ICER) was calculated by dividing the incremental costs by the incremental QALYs. The ICER represents the costs of an additional QALY gained when comparing two strategies. Whether a treatment strategy is considered cost-effective depends on how much society is willing to pay per gained QALY, which is referred to as the ceiling ratio. We adopted a ceiling ratio of $€ 80,000$, since this is the informal ceiling ratio for a high burden of disease in the Netherlands.

\section{Sensitivity analyses}

The assumption of equal disease progression for IMPT and IMRT was relaxed in a sensitivity analysis. The probabilities used in this analysis were based on a synthesis $^{6}$ of available clinical studies for oropharyngeal carcinomas (Appendix 5.3).

Probabilistic sensitivity analyses were performed to reflect the uncertainty in the input parameters and its impact on the estimated (cost-)effectiveness. ${ }^{3}$ This was done by assigning a distribution to the input parameters (Appendices 5.1-5.4) and subsequently drawing random values from these distributions using Monte Carlo simulation (20,000 iterations). The results of the probabilistic sensitivity analyses were presented using cost-effectiveness acceptability curves. For 
different ceiling ratios this curve shows the probability that a treatment strategy is cost-effective. ${ }^{3}$

\section{Value of information analyses}

As the results are surrounded by uncertainty, chances are that the wrong decision is being made when implementing the most cost-effective strategy. The expected value of perfect information (EVPI) analysis assesses the expected costs of this decision uncertainty. Hence, the EVPI places a maximum that society should be willing to pay for further evidence to reduce this uncertainty. ${ }^{3}$ The population EVPI was calculated by multiplying the EVPI per patient by the effective population in the next 10 years (expected life span of the technology) and discounted by a rate of $4 \% .^{13}$ The effective population was calculated based on a yearly incidence of 2265 HNC patients in the Netherlands (Dutch cancer registry 2008) of which 2063 were expected to receive radiotherapy, minus the estimated proportions of patients with early stage HNC (33\%) and/or pre-treatment dysphagia and/or xerostomia $\geq$ grade 2 (36\%).

To identify the most valuable research topics, the EVPI for (groups of) parameters was calculated for the NTCP models, disease progression, utility scores and costs. All analyses were performed in Microsoft Office Excel.

\section{Results}

The estimated occurrence of xerostomia and dysphagia at 12 months was lowest for IMPT for all patients (22\% and 18\%), followed by IMPT if efficient (36\% and $21 \%$ ) and IMRT for all patients (44\% and 23\%) (Appendix 5.1).

IMPT for all patients was the most effective (6.620 QALYs, 5.800 DTFLYs) and most expensive $(€ 50,989)$ strategy (Table 5.2). IMRT for all patients was the least effective (6.520 QALYs, 4.197 DTFLYs) and least expensive $(€ 41,038)$ strategy. The difference in costs between these two strategies was mainly due to higher primary treatment costs (€21,100 versus $€ 10,048)$. For all 25 patients, IMPT resulted in more QALYs compared to IMRT. Restricting IMPT to the 7 patients $(28 \%)$ for whom IMPT is expected to be cost-effective (IMPT if efficient) would yield 6.563 QALYs and 4.875 DTFLYs at an estimated cost of $€ 43,650$.

IMPT if efficient opposed to IMRT for all patients resulted in an ICER of $€ 60,278$ per QALY gained. IMPT for all patients compared with IMPT if efficient resulted in an ICER of $€ 127,946$ per QALY gained. IMPT if efficient can thus be regarded as the most cost-effective strategy (Table 5.2). 


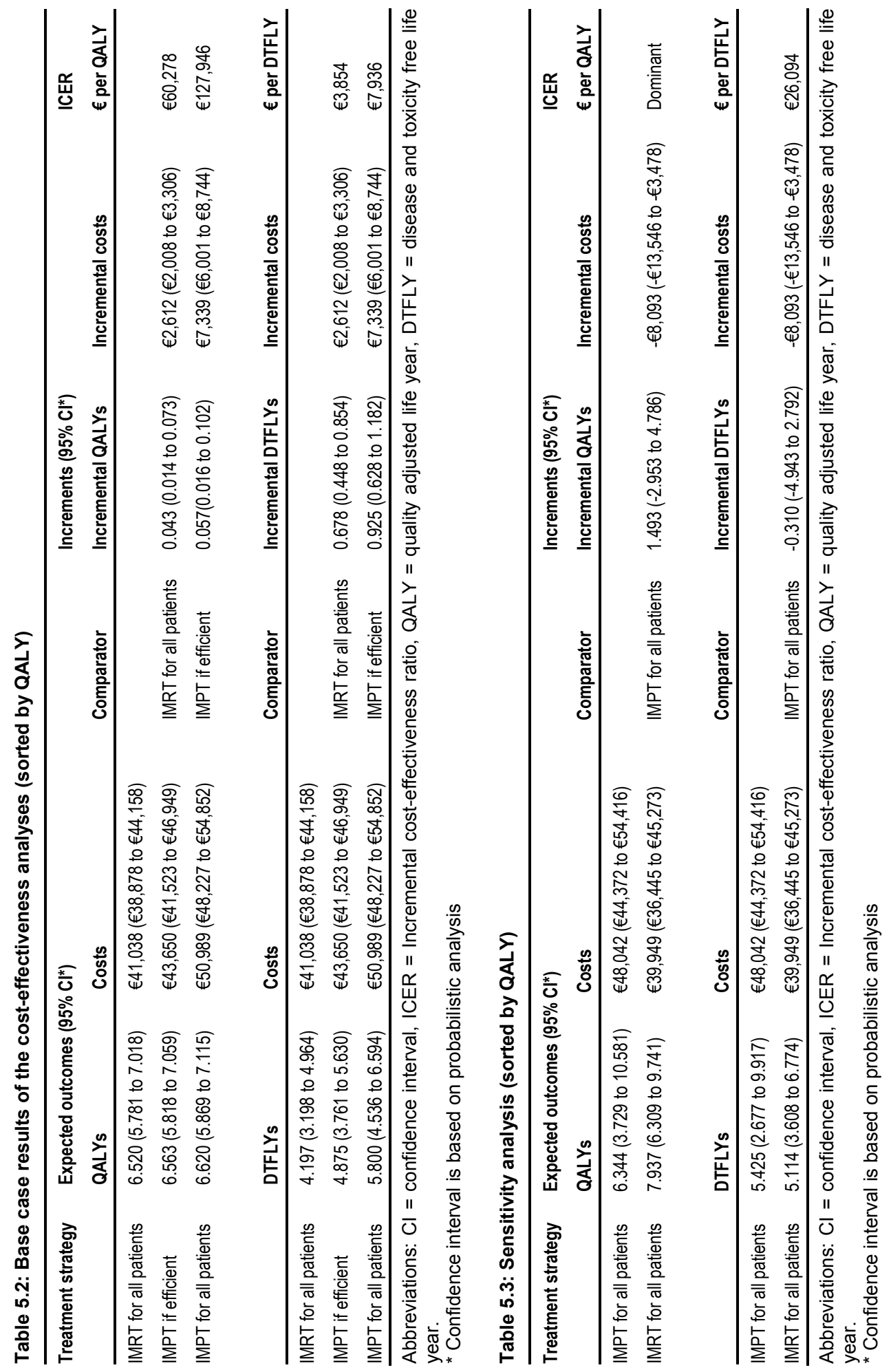


IMPT if efficient had the highest probability (62\%) of being the most costeffective strategy (Figure 5.2). The value of further research was estimated to be $€ 2,4$ million for the total population. Further research focusing on utility scores after xerostomia ( $€ 0.7$ million), NTCP models for dysphagia ( $€ 0.3$ million) and for xerostomia ( $€ 0.1$ million) is most worthwhile.

In the sensitivity analysis, IMRT for all patients yielded 1.493 more QALYs, was $€ 8,093$ less expensive and thus dominated IMPT for all patients (Table 5.3). This was the case for all individual patients. The individualized strategy was thus equal to IMRT for all patients plus the costs of an extra treatment plan and is therefore not considered in the sensitivity analysis. IMRT for all patients had the highest probability $(75 \%)$ of being cost-effective (Figure 5.2 ).

\section{Base case analysis}

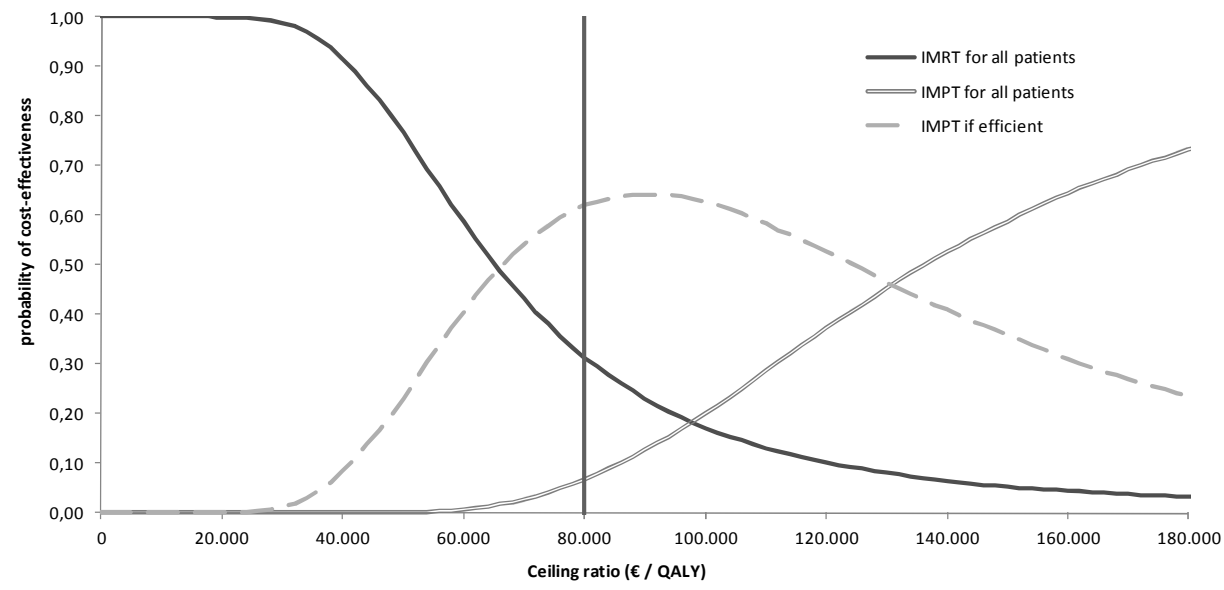

\section{Sensitivity analysis}

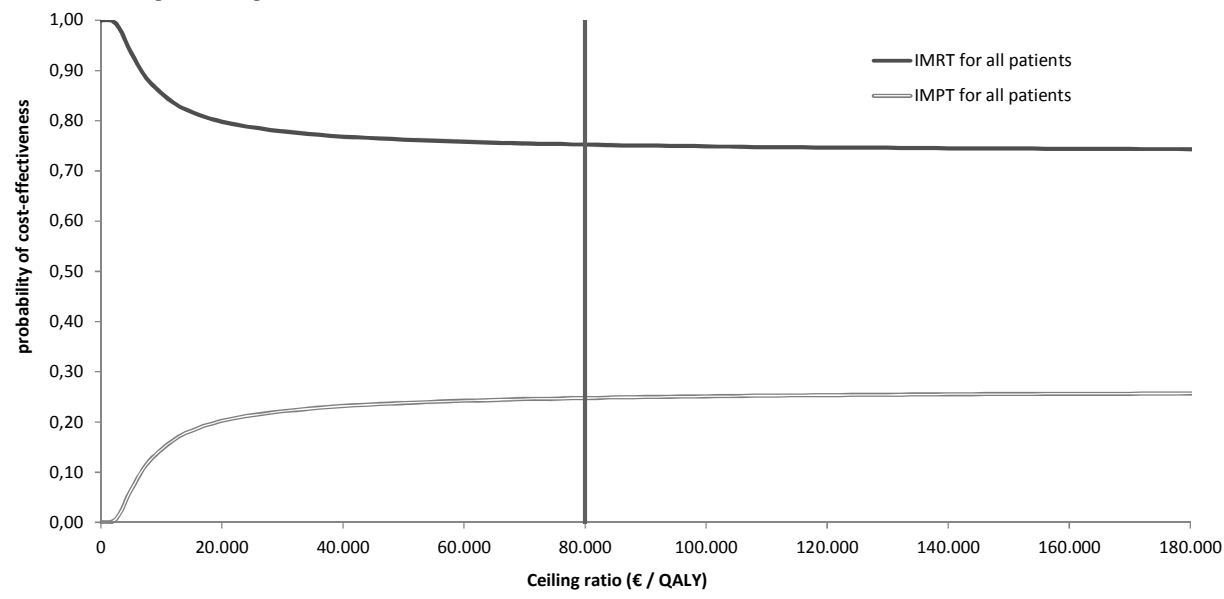

Figure 5.2: Cost-effectiveness acceptability curves

The vertical line represents the ceiling ratio which was adopted in our analyses ( $€ 80,000$ per QALY gained). 


\section{Discussion}

The original aspect of this assessment was that despite the lack of comparative effectiveness research, we were able to explore the cost-effectiveness of IMPT versus IMRT. The present study showed that employing NTCP models combined with comparative planning studies into model-based economic evaluations is feasible and informative. Besides examining the (cost-)effectiveness, this methodology can potentially be used to identify patients for whom particular treatments are more or less (cost-)effective than for the whole group. If equal disease control is assumed, IMPT is more effective than IMRT for all HNC patients. Thus, based on effectiveness, IMPT would be the treatment of choice for all patients. However, the increased effectiveness of IMPT does not outweigh its additional costs for all patients. Administering IMPT only to selected patients for whom it is expected to be cost-effective (IMPT if efficient), seems the most cost-effective treatment strategy. IMRT is the dominant strategy if disease progression is based on clinical evidence. The quality and quantity of available studies is however poor. ${ }^{6}$ Accordingly, it is possible that differences in disease progression found in these studies and thus the dominance of IMRT are not a reflection of actual differences.

Some limitations should also be discussed. Firstly, the presented methodology is not yet validated. As with any novel methodology, further research and application of the methodology in practice are needed to demonstrate its validity. Secondly, disease progression was based on a meta-analysis which included trials conducted before 2000 As recent studies show more favorable results, ${ }^{10}$ disease progression might have been overestimated. Thirdly, the health care perspective was used for cost calculation. The societal perspective, including productivity losses, might favor IMPT since less toxicity presumably reduces productivity losses. The preceding two limitations can be regarded as conservative towards IMPT. Fourthly, radiation-induced cancer was not incorporated, because evidence on the magnitude and direction of this effect is lacking. ${ }^{14}$ Also, given that radiation-induced cancer generally occurs years after radiotherapy, it is probably not an influential factor in this older population. Fifthly, utility scores for the different health states were retrieved from a cross-sectional study. ${ }^{5}$ Preferably, these utility scores are based on a prospective study to correct for possible baseline differences. However, the occurrence of xerostomia and/or dysphagia is expectedly independent of baseline utility scores. Sixthly, two available prediction models ${ }^{8,9}$ were used to predict the occurrence of toxicity. As with all prediction models, these models can possibly be optimized to achieve more accurate predictions. For the validity of the proposed methodology, it is of great importance to use valid prediction models. This uncertainty is incorporated in the probabilistic sensitivity analysis. 
Seventhly, the analyses were based on swallowing sparing radiotherapy techniques. Choice of these techniques most likely decreases the QALY gain for IMPT compared with IMRT due to a smaller reduction of dysphagia. ${ }^{7}$ Hence, using swallowing sparing techniques can be regarded as conservative towards IMPT. Those interested can use the interactive decision support tool (www.predictcancer.org) to examine the cost-effectiveness of IMPT versus IMRT for any other radiotherapy technique. Eighthly, incorporating additional time points to estimate toxicity, would lead to a more realistic representation of clinical practice. However, since this concerns both comparators, the impact on the difference in toxicity and consequently the difference in QALYs is probably small. Finally, to estimate the occurrence of toxicity, we used NTCP models validated with photons. It has been argued that using these NTCP models for protons possibly requires modification as photons and protons differ in low to intermediate dose distributions. ${ }^{15}$ At this moment, it is unknown to what degree NTCP models validated in photon studies can be used for protons.

One previous study ${ }^{16}$ addressed the cost-effectiveness of proton radiotherapy in HNC and reported a substantially lower ICER of $€ 3,811$ per QALY for proton versus photon radiotherapy than the present study. This discrepancy can be explained by differences in assumptions. Our model assumed equal survival for both comparators, whereas Lundkvist et al. assumed a mortality risk reduction of $24 \%$ for proton radiotherapy. ${ }^{16}$

To our knowledge, no other studies used NTCP models in economic evaluations. However, Konski et al. ${ }^{17}$ used dose-response data to predict recurrences in an economic evaluation for proton radiotherapy in prostate cancer.

The main research implication is that the applied study method, possibly combined with dose-response data for disease progression, ${ }^{17}$ is feasible and informative to explore the potential (cost-)effectiveness of innovative radiotherapy techniques if clinical evidence is lacking. Clinical data and especially comparative evidence is obviously superior to the proposed methodology. However, the proposed methodology offers a solution if clinical data are lacking and it is not desirable to wait or postpone decisions until clinical data become available. For proton radiotherapy this methodology may provide new insights to the debate considering pros and cons of clinical trials comparing proton and photon radiotherapy. ${ }^{4}$ It would be interesting for this debate to explore which radiotherapy technique is optimal and consider planning studies primarily focused on sparing other structures than the swallowing structures. Additionally, further research on utility scores after xerostomia and NTCP models seems most valuable to reduce decision uncertainty.

Our results showed that, based on equal survival for IMPT and IMRT, IMPT is cost-effective for individually selected patients in the Netherlands. For clinical practice it is therefore recommended to make a trade-off between expected costs and benefits for each individual patient. The presented methodology can be used to make this individual trade-off. This is in line with recent policy rec- 
ommendations. ${ }^{18}$ In these recommendations it is mentioned that a clinical significant reduction of complications is required for patients to be eligible for IMPT. However, it is not specified what can be considered as a clinical significant reduction. It is required to specify a threshold when patients are eligible for IMPT, either based on cost-effectiveness or solely on effectiveness. In our analyses, the reduction in complications was expressed in terms of QALYs. The adopted ceiling ratio of $€ 80,000$ per QALY gained enabled us to calculate which treatment is preferred based on cost-effectiveness.

In deciding which patients receive IMPT, the proposed methodology, if validated by clinical data, could act as a clinical decision support tool. Our tool is published online as example (www.predictcancer.org). Treatment allocation could then be based on individual patient data to ensure that IMPT is assigned to patients for whom it is expected to be worthwhile.

\section{Acknowledgement}

Hans-Paul van der Laan, Ivo Beetz, Miranda Christianen and Tara van de Water are gratefully acknowledged for providing additional data.

This study was supported by an unrestricted research grant (No. 152002021) from the Dutch Organization of Health Research and Development (ZonMw). ZonMw had no influence on the study design, data analyses, data interpretation, manuscript writing or the decision to submit the manuscript for publication. 


\section{References}

1. Peeters A, Grutters JP, Pijls-Johannesma M, et al: How costly is particle therapy? Cost analysis of external beam radiotherapy with carbon-ions, protons and photons. Radiother Oncol 95:45-53, 2010

2. Sullivan R, Peppercorn J, Sikora K, et al: Delivering affordable cancer care in high-income countries. Lancet Oncol 12:933-80, 2011

3. Briggs A, Sculpher MJ, Claxton K: Decision Modelling for Health Economic Evaluation. Oxford, Oxford University Press, 2006

4. De Ruysscher D, Mark Lodge M, Jones B, et al: Charged particles in radiotherapy: A 5-year update of a systematic review. Radiother Oncol 103:5-7, 2012

5. Ramaekers BL, Joore MA, Grutters JP, et al: The impact of late treatment-toxicity on generic health-related quality of life in head and neck cancer patients after radiotherapy. Oral Oncol 47:768-74, 2011

6. Ramaekers BL, Pijls-Johannesma M, Joore MA, et al: Systematic review and meta-analysis of radiotherapy in various head and neck cancers: Comparing photons, carbon-ions and protons. Cancer Treat Rev 37:185-201, 2011

7. van der Laan HP, van de Water TA, van Herpt HE, et al: The potential of intensity-modulated proton radiotherapy to reduce swallowing dysfunction in the treatment of head and neck cancer: A planning comparative study. Acta Oncol 52:561-9, 2013

8. Beetz I, Schilstra C, Burlage FR, et al: Dose distributions in the minor salivary glands and the development of radiation-induced patient-rated xerostomia. Radioth Oncol 96:S84-S85, 2010

9. Christianen ME, Schilstra C, Beetz I, et al: Predictive modelling for swallowing dysfunction after primary (chemo)radiation: Results of a prospective observational study. Radiother Oncol 105:107-14, 2012

10. Pignon JP, le Maitre A, Maillard E, et al: Meta-analysis of chemotherapy in head and neck cancer (MACH-NC): an update on 93 randomised trials and 17,346 patients. Radiother Oncol 92:4-14, 2009

11. Goitein $M$, Jermann $M$ : The relative costs of proton and X-ray radiation therapy. Clin Oncol $(R$ Coll Radiol) 15:S37-50, 2003

12. Hunink MG, Glasziou PP: Decision Making in Health and Medicine: Integrating Evidence and Values. Cambridge, Cambridge University Press, 2001

13. Health Care Insurance Board: Guidelines for pharmaco-economic research [in Dutch]. Diemen, Health Care Insurance Board 2006

14. Hall EJ: Intensity-modulated radiation therapy, protons, and the risk of second cancers. Int J Radiat Oncol Biol Phys 65:1-7, 2006

15. Michalski JM, Gay H, Jackson A, et al: Radiation dose-volume effects in radiation-induced rectal injury. Int J Radiat Oncol Biol Phys 76:S123-9, 2010

16. Lundkvist J, Ekman M, Ericsson SR, et al: Proton therapy of cancer: potential clinical advantages and cost-effectiveness. Acta Oncol 44:850-61, 2005

17. Konski A, Speier W, Hanlon A, et al: Is proton beam therapy cost effective in the treatment of adenocarcinoma of the prostate? J Clin Oncol 25:3603-8, 2007

18. Health Care Insurance Board: Indications for proton therapy (part 2): model-based indications [In Dutch]. Diemen, Health Care Insurance Board, 2011 


\section{Appendix 5.1: NTCP models, planning studies and toxicity probabilities}

\section{NTCP models}

Probability RTOG $\geq$ grade 2 xerostomia

6 months after radiotherapy $(\mathrm{N}=354)$

Constant

Mean dose to parotis ipsilateral

Mean dose to parotis contralateral

Probability RTOG $\geq$ grade 2 xerostomia

12 months after radiotherapy $(\mathrm{N}=251)$

Constant

Mean dose to parotis ipsilateral

Mean dose to parotis contralateral

Probability RTOG $\geq$ grade 2 dysphagia

6 months after radiotherapy $(\mathrm{N}=354)$

Constant

Mean dose to pharyngeal constrictor muscle superior

Mean dose to supraglottic area

Probability RTOG $\geq$ grade 2 dysphagia

12 months after radiotherapy $(\mathrm{N}=230)$

Constant

Mean dose to pharyngeal constrictor muscle superior

Mean dose to supraglottic area

$\begin{array}{lll}\text { Coefficient } & \text { Se } & \text { Distribution } \\ -2.852 & 0.313 & \text { Multivariate normal }^{*_{1}} \\ 0.031 & 0.009 & \text { Multivariate normal }^{*_{1}} \\ 0.036 & 0.010 & \text { Multivariate normal }^{*_{1}}\end{array}$

Coefficient Se Distribution Source

-2.9490 .386 Multivariate normal ${ }^{{ }^{*} 1}$

0.0250 .011 Multivariate normal ${ }^{{ }_{1}}$

0.0450 .012 Multivariate normal ${ }^{\star 1}$

Coefficient Se Distribution Source

$-6.094 \quad 0.925$ Multivariate normal ${ }^{*_{2}}$

$0.057 \quad 0.009$ Multivariate normal ${ }^{*_{2}}$

$0.037 \quad 0.012$ Multivariate normal ${ }^{{ }^{2}}$

Coefficient Se Distribution Source

$-6.343 \quad 1.186$ Multivariate normal ${ }^{*_{2}}$

$0.058 \quad 0.012$ Multivariate normal ${ }^{*_{2}}$

$0.027 \quad 0.015$ Multivariate normal ${ }^{{ }^{2}}$

Radiation dose to healthy tissues based on comparative planning study (GyE)

\begin{tabular}{lllllllllll}
\hline & IMRT & \multicolumn{2}{c}{ Range $^{\dagger}$} & & IMPT & Range $^{\dagger}$ & & Source \\
Mean dose to parotis ipsilateral & 45.4 & 25.6 & - & 65.0 & 28.5 & 15.2 & - & 48.6 & 3 \\
Mean dose to parotis contralateral & 35.0 & 24.3 & - & 48.3 & 21.2 & 6.5 & - & 30.7 & 3 \\
Mean dose to pharyngeal constrictor & & & & & & & & & \\
muscle superior & 62.6 & 50.3 & - & 70.8 & 61.6 & 44.5 & - & 70.1 & 3 \\
Mean dose to supraglottic area & 53.4 & 25.3 & - & 70.3 & 40.3 & 10.0 & - & 70.3 & 3
\end{tabular}

Overlap between RTOG $\geq$ grade 2 xerostomia and RTOG $\geq$ grade 2 dysphagia

Distribution Source

Probability of dysphagia conditional on having xerostomia

$\mathrm{P}$ (dysphagia | xerostomia)

$55.8 \%$

Fixed $\quad 4$

Probability of xerostomia conditional on having dysphagia

$\mathrm{P}$ (xerostomia | dysphagia)

$65.9 \%$

Fixed

Calculated average probabilities of RTOG $\geq$ grade 2 xerostomia and dysphagia without taking into account overlap $(\%)^{\ddagger}$

\begin{tabular}{|c|c|c|c|c|c|c|}
\hline & IMRT & Range & & IMPT & Range $^{\dagger}$ & \\
\hline RTOG $\geq$ grade 2 xerostomia ( 6 months) & 45.8 & 23.6 & 65.7 & 23.8 & 10.6 & 38.5 \\
\hline RTOG $\geq$ grade 2 xerostomia (12 months) & 44.5 & 23.1 & 64.8 & 22.5 & 9.4 & 36.6 \\
\hline RTOG $\geq$ grade 2 dysphagia (6 months) & 37.6 & 17.7 & 62.0 & 29.9 & 4.3 & 61.9 \\
\hline RTOG $\geq$ grade 2 dysphagia ( 12 months) & 23.0 & 11.1 & 40.7 & 18.4 & 3.2 & 40.3 \\
\hline
\end{tabular}




\section{Appendix 5.1 (continued): NTCP models, planning studies and toxicity probabilities}

\section{Input parameters for Markov model}

Calculated probabilities of RTOG $\geq$ grade 2 xerostomia and RTOG $\geq$ grade 2 dysphagia taking into account overlap (\%)

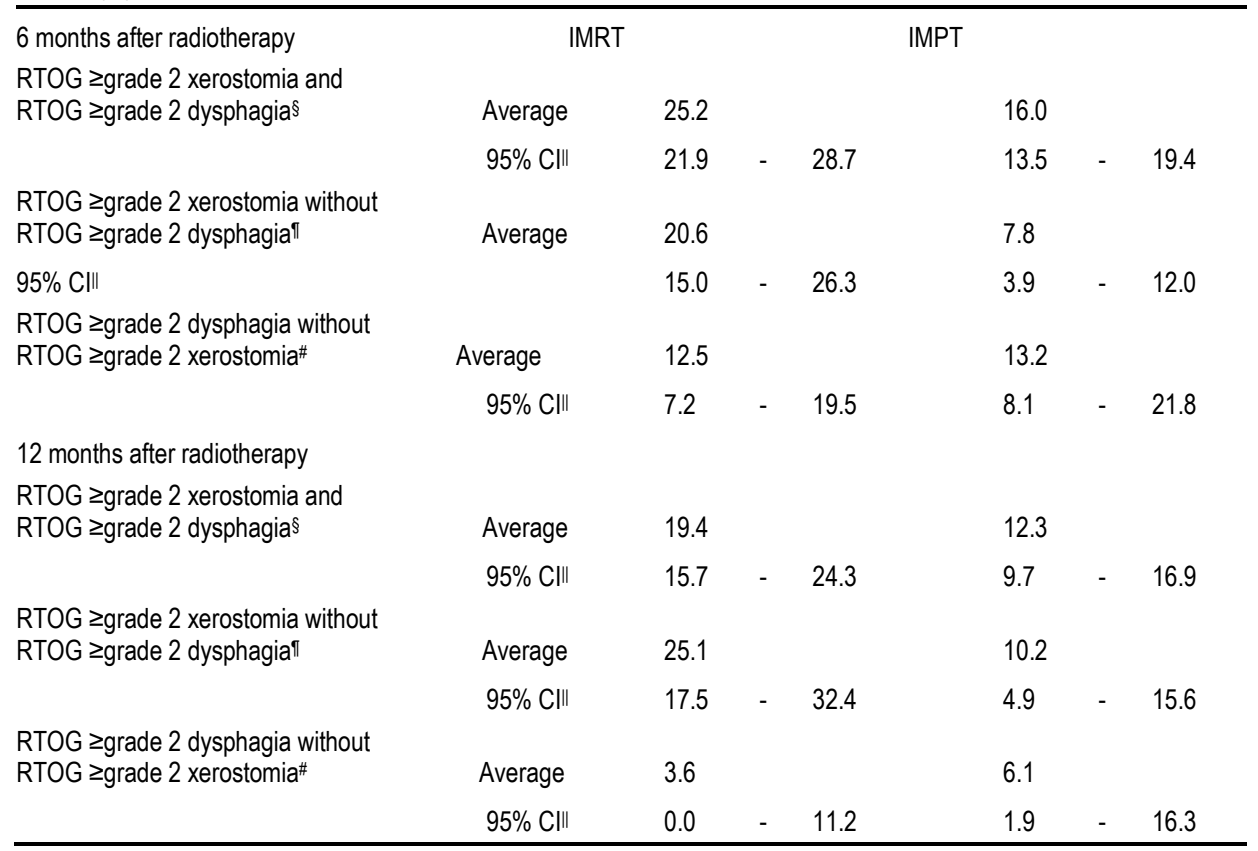

Abbreviations: $\mathrm{Se}=$ standard error, $\mathrm{Cl}=$ confidence interval, NTCP = normal tissue complication probability, IMRT = intensity-modulated radiotherapy with photons, IMPT = intensity-modulated proton radiotherapy, RTOG = Radiation Therapy Oncology Group, GyE = gray equivalent

${ }^{*}$ The multivariate normal distribution was constructed using Cholesky decompositions ${ }^{5}$

$\dagger$ The range of point estimates across all 25 patients.

$\ddagger$ The average toxicity probability was calculated based on the individual toxicity probabilities per patient.

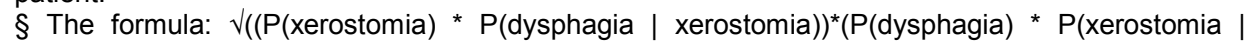
dysphagia))) was used to calculate the probability that patients had both xerostomia and dysphagia. II The 95\% confidence interval represents the parameter uncertainty accompanying the point estimate, and was generated in the probabilistic sensitivity analysis.

I The formula: $\mathrm{P}$ (xerostomia) - $\mathrm{P}$ (xerostomia and dysphagia) was used to calculate the probability that patients had xerostomia without dysphagia

\# The formula: $\mathrm{P}$ (dysphagia) - $\mathrm{P}$ (xerostomia and dysphagia) was used to calculate the probability that patients had dysphagia without xerostomia 


\section{Appendix 5.2: Toxicity probabilities for the all strategies}

\section{Input parameters for Markov model}

Calculated probabilities of RTOG $\geq$ grade 2 xerostomia and RTOG $\geq$ grade 2 dysphagia taking into account overlap $(\%)^{\dagger}$

\begin{tabular}{|c|c|c|c|c|c|c|c|}
\hline \multicolumn{2}{|l|}{6 months after radiotherapy } & \multicolumn{2}{|c|}{ IMRT for all patients } & \multicolumn{2}{|c|}{ IMPT for all patients } & \multicolumn{2}{|c|}{ IMPT if efficient } \\
\hline $\begin{array}{l}\text { RTOG } \geq \text { grade } 2 \text { xerostomia and } \\
\text { RTOG } \geq \text { grade } 2 \text { dysphagia }\end{array}$ & Average & 25.2 & & 16.0 & & 21.2 & \\
\hline & $95 \% \mathrm{Cl}$ & 21.9 & -28.7 & 13.5 & -19.4 & 18.3 & -24.9 \\
\hline \multicolumn{8}{|l|}{ RTOG $\geq$ grade 2 xerostomia without } \\
\hline \multirow[t]{2}{*}{ RTOG $\geq$ grade 2 dysphagia } & Average & 20.6 & & 7.8 & & 15.9 & \\
\hline & $95 \% \mathrm{Cl}$ & 15.0 & -26.3 & 3.9 & -12.0 & 11.0 & -20.8 \\
\hline \multirow[t]{2}{*}{$\begin{array}{l}\text { RTOG } \geq \text { grade } 2 \text { dysphagia without } \\
\text { RTOG } \geq \text { grade } 2 \text { xerostomia }\end{array}$} & Average & 12.5 & & 13.2 & & 11.8 & \\
\hline & $95 \% \mathrm{Cl}$ & 7.2 & -19.5 & 8.1 & -21.8 & 6.8 & -19.5 \\
\hline \multicolumn{8}{|l|}{12 months after radiotherapy } \\
\hline \multirow[t]{2}{*}{$\begin{array}{l}\text { RTOG } \geq \text { grade } 2 \text { xerostomia and } \\
\text { RTOG } \geq \text { grade } 2 \text { dysphagia }\end{array}$} & Average & 19.4 & & 12.3 & & 16.4 & \\
\hline & $95 \% \mathrm{Cl}$ & 15.7 & -24.3 & 9.7 & -16.9 & 13.2 & -21.5 \\
\hline \multirow[t]{2}{*}{$\begin{array}{l}\text { RTOG } \geq \text { grade } 2 \text { xerostomia without } \\
\text { RTOG } \geq \text { grade } 2 \text { dysphagia }\end{array}$} & Average & 25.1 & & 10.2 & & 19.2 & \\
\hline & $95 \% \mathrm{Cl}^{\ddagger}$ & 17.5 & -32.4 & 4.9 & -15.6 & 12.5 & -25.6 \\
\hline \multirow[t]{2}{*}{$\begin{array}{l}\text { RTOG } \geq \text { grade } 2 \text { dysphagia without } \\
\text { RTOG } \geq \text { grade } 2 \text { xerostomia }\end{array}$} & Average & 3.6 & & 6.1 & & 4.2 & \\
\hline & $95 \% \mathrm{Cl}$ & 00 & -112 & 1.9 & -16.3 & 0.3 & -13.3 \\
\hline
\end{tabular}

Abbreviations: $\mathrm{Cl}=$ confidence interval, IMRT = intensity-modulated

* The average toxicity probability was calculated based on the individual toxicity probabilities per patient instead of average dose parameters

† See Appendix 5.1 for calculation steps

$\ddagger$ The confidence interval represents the parameter uncertainty accompanying the point estimate, and was generated in the probabilistic sensitivity analyses. 


\section{Appendix 5.3: Treatment independent transition probabilities and health state utility scores}

\begin{tabular}{|c|c|c|c|c|}
\hline Parameter & $\begin{array}{l}\text { Estimated } \\
\text { value }\end{array}$ & $\mathrm{Se}$ & Distribution & Source \\
\hline \multicolumn{5}{|l|}{ Transition probabilities (\%) } \\
\hline \multicolumn{5}{|l|}{ From disease free with or without toxicity } \\
\hline Loco-regional recurrence 0-2 year after radiotherapy* & 0.256 & 0.003 & Beta & 6 \\
\hline Loco-regional recurrence 2-5 year after radiotherapy & 0.038 & 0.006 & Beta & 6 \\
\hline Loco-regional recurrence more than 5 year after radiotherapy ${ }^{\dagger}$ & 0.010 & 0.011 & Beta & 6 \\
\hline Distant metastases $0-2$ year after radiotherapy* & 0.058 & 0.002 & Beta & 6 \\
\hline Distant metastases 2-5 year after radiotherapy & 0.021 & 0.003 & Beta & 6 \\
\hline Distant metastases more than 5 year after radiotherapy ${ }^{\dagger}$ & 0.003 & 0.004 & Beta & 6 \\
\hline \multicolumn{5}{|l|}{ Cancer-related mortality from: } \\
\hline Loco-regional recurrence* & 0.480 & 0.051 & Beta & 7 \\
\hline Distant metastases & 1.000 & Fixed & - & ${ }^{8}$ and Eo \\
\hline \multicolumn{5}{|l|}{ Sensitivity analysis } \\
\hline \multicolumn{5}{|l|}{ 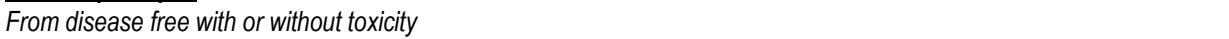 } \\
\hline \multicolumn{5}{|l|}{ IMRT } \\
\hline Loco-regional recurrence $0-2$ year after radiotherapy* & 0.027 & 0.013 & Beta & 9 \\
\hline Loco-regional recurrence 2-5 year after radiotherapy ${ }^{\ddagger}$ & 0.027 & 0.013 & Beta & 9 \\
\hline Loco-regional recurrence more than 5 year after radiotherapy $\ddagger$ & 0.027 & 0.013 & Beta & 9 \\
\hline Distant metastases§ 0-2 year after radiotherapy* & 0.042 & 0.019 & Beta & 9 \\
\hline Distant metastases§ $2-5$ year after radiotherapy $\ddagger$ & 0.042 & 0.019 & Beta & 9 \\
\hline Distant metastases§ more than 5 year after radiotherapy $\ddagger$ & 0.042 & 0.019 & Beta & 9 \\
\hline \multicolumn{5}{|l|}{ IMPT } \\
\hline Loco-regional recurrence 0-2 year after radiotherapy* & 0.020 & 0.022 & Beta & 9 \\
\hline Loco-regional recurrence 2-5 year after radiotherapy $\ddagger$ & 0.020 & 0.022 & Beta & 9 \\
\hline Loco-regional recurrence more than 5 year after radiotherapy $\ddagger$ & 0.020 & 0.022 & Beta & 9 \\
\hline Distant metastases§ 0-2 year after radiotherapy* & 0.078 & 0.046 & Beta & 9 \\
\hline Distant metastases $\$ 2-5$ year after radiotherapy $\ddagger$ & 0.078 & 0.046 & Beta & 9 \\
\hline Distant metastases§ more than 5 year after radiotherapy $\ddagger$ & 0.078 & 0.046 & Beta & 9 \\
\hline \multicolumn{5}{|l|}{ Health state utility scores } \\
\hline Disease free, no toxicity & 0.880 & 0.010 & Beta & 4 \\
\hline Disease free, xerostomia $\geq$ grade 2 & 0.826 & 0.027 & Beta & 4 \\
\hline Disease free, dysphagia $\geq$ grade 2 & 0.803 & 0.036 & Beta & 4 \\
\hline Disease free, xerostomia $\geq$ grade 2 and dysphagia $\geq$ grade 2 & 0.761 & 0.029 & Beta & 4 \\
\hline Loco-regional disease and distant disease & 0.720 & 0.070 & Beta & 4 \\
\hline
\end{tabular}

Abbreviations: $\mathrm{Se}=$ standard error, Eo $=$ expert opinion

* The 6 months probability was calculated from the yearly probability using the following formula ${ }^{5}$ : $P(6$ months $)=1-\mathrm{e}^{\left(\ln (1-P(1 \text { year }))^{*} 0.5\right)}$

$\dagger$ The yearly loco-regional and distant failure probabilities between 5 - and 7-year were assumed to remain constant for life-time.

$\ddagger$ Assumed equal as the 0-2 year probability

$\S$ Calculated by subtracting the loco-regional control probabilities from the disease free survival probabilities $^{9}$ 
Appendix 5.4: Resource use and unit prices

\begin{tabular}{|c|c|c|c|c|c|}
\hline \multirow{2}{*}{$\begin{array}{l}\text { Parameter } \\
\text { Number of speech therapist examinations }\end{array}$} & \multirow{2}{*}{$\begin{array}{l}\begin{array}{l}\text { Estimated } \\
\text { value }\end{array} \\
1.0\end{array}$} & \multicolumn{2}{|c|}{ Range / Se } & \multirow{2}{*}{$\begin{array}{l}\text { Distribution } \\
-\end{array}$} & \multirow{2}{*}{$\begin{array}{l}\text { Source } \\
\text { Eo }\end{array}$} \\
\hline & & Fixed & & & \\
\hline Number of speech therapist consults & 8.0 & $6.0-$ & 10.0 & Beta PERT & Eo \\
\hline Radiation technician hours per treatment plan & 3.0 & $1.0-$ & 6.0 & Beta PERT & Eo \\
\hline $\begin{array}{l}\text { Number of follow-up consults per year } \\
\text { (year } 1 \text { after primary treatment/re-treatment) }\end{array}$ & 5.0 & Fixed & & - & 10 \\
\hline $\begin{array}{l}\text { Number of follow-up consults per year } \\
\text { (year } 2 \text { after primary treatment/re-treatment) }\end{array}$ & 4.0 & Fixed & & - & 10 \\
\hline $\begin{array}{l}\text { Number of follow-up consults per year } \\
\text { (year } 3 \text { after primary treatment/re-treatment) }\end{array}$ & 2.5 & Fixed & & - & 10 \\
\hline $\begin{array}{l}\text { Number of follow-up consults per year } \\
\text { (year } 4 \text { after primary treatment/re-treatment) }\end{array}$ & 2.0 & Fixed & & - & 10 \\
\hline $\begin{array}{l}\text { Number of follow-up consults per year } \\
\text { (year } 5 \text { after primary treatment/re-treatment) }\end{array}$ & 2.0 & Fixed & & - & 10 \\
\hline \multicolumn{6}{|l|}{ Related to xerostomia grade $0-1$} \\
\hline Number of fillings & 2.3 & 0.8 & & Gamma & 4 \\
\hline Number of crowns & 7.5 & 5.5 & & Gamma & 4 \\
\hline Number of fixed partial dentures & 1.5 & 0.5 & & Gamma & 4 \\
\hline Number of extra dentist consults per year & 0.0 & Fixed & & - & 11 \\
\hline Gram Natriumfluoride gel per year & 0.0 & Fixed & & - & Eo \\
\hline$\%$ of patients who had fillings ${ }^{\dagger}$ & $4.0 \%$ & $1.2 \%$ & & Beta & 4 \\
\hline$\%$ of patients with crowns ${ }^{\dagger}$ & $1.0 \%$ & $0.6 \%$ & & Beta & 4 \\
\hline$\%$ of patients who had fixed partial dentures ${ }^{\dagger}$ & $1.0 \%$ & $0.6 \%$ & & Beta & 4 \\
\hline$\%$ of patients who had root canal treatment ${ }^{\dagger}$ & $2.0 \%$ & $0.8 \%$ & & Beta & 4 \\
\hline$\%$ of patients who had dental plates ${ }^{\dagger}$ & $10.0 \%$ & $1.8 \%$ & & Beta & 4 \\
\hline$\%$ of patients who had dental plates ${ }^{\dagger}$ & $7.0 \%$ & $1.5 \%$ & & Beta & 4 \\
\hline$\%$ of patients who had dental implants ${ }^{\dagger}$ & $1.0 \%$ & $0.7 \%$ & & Beta & 4 \\
\hline$\%$ of patients who had dental implants ${ }^{\dagger}$ & $2.0 \%$ & $0.8 \%$ & & Beta & 4 \\
\hline$\%$ of patients with extra dentist consults & $0.0 \%$ & Fixed & & - & 11 \\
\hline$\%$ of patients with Natriumfluoride gel & $0.0 \%$ & Fixed & & - & 10 \\
\hline \multicolumn{6}{|l|}{ Related to xerostomia $\geq$ grade 2} \\
\hline Number of fillings & 3.0 & 0.6 & & Gamma & 4 \\
\hline Number of crowns & 0.0 & 0.0 & & Gamma & 4 \\
\hline Number of fixed partial dentures & 0.0 & 0.0 & & Gamma & 4 \\
\hline Number of extra dentist consults per year & 2.0 & Fixed & & - & 11 \\
\hline Gram Natriumfluoride gel per year & 365.0 & Fixed & & - & Eo \\
\hline$\%$ of patients who had fillings ${ }^{\dagger}$ & $7.0 \%$ & $3.0 \%$ & & Beta & 4 \\
\hline$\%$ of patients with crowns ${ }^{\dagger}$ & $0.0 \%$ & $0.0 \%$ & & Beta & 4 \\
\hline$\%$ of patients who had fixed partial dentures ${ }^{\dagger}$ & $0.0 \%$ & $0.0 \%$ & & Beta & 4 \\
\hline$\%$ of patients who had root canal treatment ${ }^{\dagger}$ & $0.0 \%$ & $0.0 \%$ & & Beta & 4 \\
\hline$\%$ of patients who had dental plates ${ }^{\dagger}$ & $11.0 \%$ & $3.0 \%$ & & Beta & 4 \\
\hline$\%$ of patients who had dental plates ${ }^{\dagger}$ & $8.0 \%$ & $3.0 \%$ & & Beta & 4 \\
\hline$\%$ of patients who had dental implants ${ }^{\dagger}$ & $1.0 \%$ & $1.0 \%$ & & Beta & 4 \\
\hline$\%$ of patients who had dental implants ${ }^{\dagger}$ & $3.0 \%$ & $2.0 \%$ & & Beta & 4 \\
\hline$\%$ of patients with extra dentist consults & $100.0 \%$ & Fixed & & - & 11 \\
\hline$\%$ of patients with Natriumfluoride gel & $100.0 \%$ & Fixed & & - & 10 \\
\hline
\end{tabular}




\section{Appendix 5.4 (continued): Resource use and unit prices}

Related to dysphagia grade $0-1$

Portions of liquid nutrition per day

Liter of tube feeding per year

$\%$ of patients with liquid nutrition

$\%$ for patients with tube feeding

Related to dysphagia $\geq$ grade 2

Portions of liquid nutrition per day

Liter of tube feeding per year

$\%$ of patients with liquid nutrition

$\%$ for patients with tube feeding

\section{$\underline{\text { Unit prices }(€)^{ \pm}}$}

Cost ratio for IMPT (primary treatment)

Cost of IMRT (primary treatment)

Cost per speech therapist examination

Cost per speech therapist consult

Cost per radiotherapist consult

Cost per dentist consult

Cost of radiation technician (per hour)

Costs per 100 gram Natriumfluoride gel ( $1 \%$ LNA)

Costs per portion of Liquid nutrition (125 mL)

Costs per $125 \mathrm{~mL}$ of tube feeding

Costs of placing and removing tube

Costs per filling

Costs per crown

Costs per fixed partial denture

Costs per root canal treatment

Costs per dental plate (upper or lower teeth)

Costs per dental implant (upper or lower teeth)

Other costs (€)

Cost of salvage therapy after loco-regional recurrence

Costs of cancer death

Costs of non-cancer death

$\begin{array}{ll}2.7 & 0.3 \\ 0.0 & 0.0 \\ 11.0 \% & 2.0 \% \\ 0.0 \% & 0.0 \%\end{array}$

$3.7 \quad 0.3$

$1237.5 \quad 151.9$

$56.0 \% \quad 5.0 \%$

$15.0 \% \quad 4.0 \%$

$\begin{array}{llll}2.1 & 1.8-2.4 & \text { Beta PERT } & 12,13 \\ 10,036.49 & \text { Fixed } & - & 14 \\ 44.49 & \text { Fixed } & - & 15 \\ 22.25 & \text { Fixed } & - & 15 \\ 62.16 & \text { Fixed } & - & 14 \\ 19.47 & \text { Fixed } & - & 15 \\ 29.49 & \text { Fixed } & & 12 \\ 7.55 & \text { Fixed } & - & \text { MP } \\ 3.70 & \text { Fixed } & - & \text { MP } \\ 2.76 & \text { Fixed } & - & \text { MP } \\ 210.91 & \text { Fixed } & - & 14 \\ 76.87 & \text { Fixed } & - & \text { Appendix 5.5 } \\ 586.21 & \text { Fixed } & - & \text { Appendix 5.5 } \\ 1,595.95 & \text { Fixed } & - & \text { Appendix 5.5 } \\ 271.74 & \text { Fixed } & - & \text { Appendix 5.5 } \\ 463.09 & \text { Fixed } & - & \text { Appendix 5.5 } \\ 2,339.45 & \text { Fixed } & - & \text { Appendix 5.5 }\end{array}$

Abbreviations: $\mathrm{Se}=$ standard error, $\mathrm{Eo}=$ expert opinion, $\mathrm{MP}=$ market price retrieved from http://www.efarma.nl/ or http://www.sorgente.nl/

* These resources were once only

$\dagger$ In the last 6 months

$\ddagger$ All unit prices were converted to the 2010 price level based on price indices ${ }^{18}$ 


\section{Appendix 5.5: Unit prices dentist costs}

\begin{tabular}{|c|c|c|c|c|}
\hline Item & Code & Procedure & Costs $(€)$ & Source \\
\hline & A10 & Anesthesia & 12.81 & 15 \\
\hline & V12 & Restoration & 33.31 & 15 \\
\hline & V21 & Cauterization and foundation & 20.50 & 15 \\
\hline & V50 & Cofferdam & 10.25 & 15 \\
\hline \multirow[t]{7}{*}{ Costs per filling } & & & 76.87 & \\
\hline & $\mathrm{X} 10$ & Radiograph & 14.35 & 15 \\
\hline & $\mathrm{A} 10$ & Anesthesia (2x) & 25.62 & 15 \\
\hline & R31 & Plastic construction & 30.74 & 15 \\
\hline & V21 & Cauterization and foundation & 20.50 & 15 \\
\hline & $\mathrm{R} 25$ & Dentist tariff crown & 225.00 & 15 \\
\hline & $\mathrm{R} 00$ & Dental technique costs & 270.00 & 15 \\
\hline \multirow[t]{8}{*}{ Costs per crown } & & & 586.21 & \\
\hline & $\mathrm{X} 10$ & Radiograph & 14.35 & 15 \\
\hline & A10 & Anesthesia (2x) & 25.62 & 15 \\
\hline & V21 & Cauterization and foundation $(2 \mathrm{x})$ & 41.00 & 15 \\
\hline & R31 & Plastic construction $(2 \mathrm{x})$ & 61.48 & 15 \\
\hline & $\mathrm{R} 25$ & Dentist tariff crown $(2 \mathrm{x})$ & 450.00 & 15 \\
\hline & $\mathrm{R} 40$ & Dentist tariff between part & 153.50 & 15 \\
\hline & $\mathrm{R} 00$ & Dental technique costs & 850.00 & 15 \\
\hline \multirow[t]{8}{*}{ Costs per fixed partial denture } & & & $1,595.95$ & \\
\hline & A10 & Anesthesia $(2 x)$ & 25.62 & 15 \\
\hline & $\mathrm{X} 10$ & Radiograph (4x) & 57.40 & 15 \\
\hline & V50 & Cofferdam $(2 x)$ & 20.50 & 15 \\
\hline & E85 & Electronic determination of length & 12.81 & 15 \\
\hline & E13 & Single-canal element & 92.23 & 15 \\
\hline & E01 & Dentist consult & 19.47 & 15 \\
\hline & E04 & Surcharge instrumentation costs & 43.71 & 15 \\
\hline \multirow[t]{4}{*}{ Costs per root canal treatment } & & & 271.74 & \\
\hline & P15 & Partial plate prosthesis $5-13$ elements & 153.72 & 15 \\
\hline & P16 & Individual print & 56.37 & 15 \\
\hline & R00 & Dental technique costs & 253.00 & 15 \\
\hline \multirow[t]{13}{*}{ Costs per dental plate } & & & 463.09 & \\
\hline & $\mathrm{X} 21$ & Orthopantomogram & 61.49 & 15 \\
\hline & J01 & Initial examination implantodontics & 66.61 & 15 \\
\hline & $\mathrm{J} 02$ & Extended examination implantodontics & 102.48 & 15 \\
\hline & J97 & Overhead costs implants & 188.50 & 15 \\
\hline & $\mathrm{J} 20$ & Placing first implant & 199.84 & 15 \\
\hline & $\mathrm{J} 23$ & 2nd phase operation & 76.86 & 15 \\
\hline & $\mathrm{J} 44$ & Abutment for crown & 25.62 & 15 \\
\hline & $\mathrm{R} 25$ & Dentist tariff crown & 225.00 & 15 \\
\hline & $\mathrm{X} 10$ & Radiograph (3x) & 43.05 & 15 \\
\hline & $\mathrm{J} 00$ & Material costs implantodontics & 500.00 & 15 \\
\hline & $\mathrm{R} 00$ & Dental technique costs & 450.00 & 15 \\
\hline & - & Bone reconstruction procedure & 400.00 & 15 \\
\hline Costs per dental implant & & & $2,339.45$ & \\
\hline
\end{tabular}




\section{Appendix references}

1. Beetz I, Schilstra C, Burlage FR, et al: Dose distributions in the minor salivary glands and the development of radiation-induced patient-rated xerostomia. Radioth Oncol 96:S84-S85, 2010

2. Christianen ME, Schilstra C, Beetz I, et al: Predictive modelling for swallowing dysfunction after primary (chemo)radiation: Results of a prospective observational study. Radiother Oncol 105:107-14, 2012

3. van der Laan HP, van de Water TA, van Herpt HE, et al: The potential of intensity-modulated proton radiotherapy to reduce swallowing dysfunction in the treatment of head and neck cancer: A planning comparative study. Acta Oncol 52:561-9, 2013

4. Ramaekers BL, Joore MA, Grutters JP, et al: The impact of late treatment-toxicity on generic health-related quality of life in head and neck cancer patients after radiotherapy. Oral Oncol 47:768-74, 2011

5. Briggs A, Sculpher MJ, Claxton K: Decision Modelling for Health Economic Evaluation. Oxford, Oxford University Press, 2006

6. Pignon JP, le Maitre A, Maillard E, et al: Meta-analysis of chemotherapy in head and neck cancer (MACH-NC): an update on 93 randomised trials and 17,346 patients. Radiother Oncol 92:4-14, 2009

7. Spencer SA, Harris J, Wheeler RH, et al: Final report of RTOG 9610, a multi-institutional trial of reirradiation and chemotherapy for unresectable recurrent squamous cell carcinoma of the head and neck. Head Neck 30:281-8, 2008

8. Kowalski LP, Carvalho AL: Natural history of untreated head and neck cancer. Eur J Cancer 36:1032-7, 2000

9. Ramaekers BL, Pijls-Johannesma M, Joore MA, et al: Systematic review and meta-analysis of radiotherapy in various head and neck cancers: Comparing photons, carbon-ions and protons. Cancer Treat Rev 37:185-201, 2011

10. Nederlandse Werkgroep Hoofd-Halstumoren [Dutch working group of Head and neck tumors]. Guideline Oral cavity and oropharyngeal carcinoma [in Dutch]. Kwaliteitsinstituut voor de Gezondheidszorg Centraal Begeleidings Orgaan, 2004.

11. Ivoren kruis: Droge mond [Dry mouth], 2005

12. Peeters A, Grutters JP, Pijls-Johannesma M, et al: How costly is particle therapy? Cost analysis of external beam radiotherapy with carbon-ions, protons and photons. Radiother Oncol 95:45-53, 2010

13. Goitein $M$, Jermann $M$ : The relative costs of proton and X-ray radiation therapy. Clin Oncol (R Coll Radiol) 15:S37-50, 2003

14. Oostenbrink JB, Koopmanschap MA, Rutten FFH. Manual for costing research [in Dutch]. Amstelveen: College voor zorgverzekeringen, 2004.

15. Nederlandse Zorgautoriteit. List of tariffs [in Dutch]. Utrecht: Nederlandse Zorgautoriteit 2009.

16. Van Agthoven M, Van Ineveld BM, De Boer MF, et al: The costs of head and neck oncology: primary tumours, recurrent tumours and long-term follow-up. Eur J Cancer 37:2204-11, 2001

17. Kommer GJ, Slobbe LCJ, Polder JJ. Risicosolidariteit en zorgkosten. Council for Public Health and Health Care (RVZ) 2005.

18. Centraal Bureau voor de Statistiek [Statistic Netherlands]: Price indeces: http://statline.cbs.nl/ 


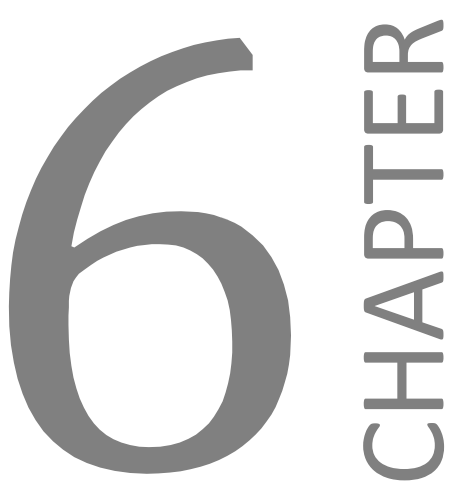

COST-EFFECTIVENESS OF MODIFIED

FRACTIONATION RADIOTHERAPY VERSUS

CONVENTIONAL RADIOTHERAPY FOR UNRESECTED

NON-SMALL CELL LUNG CANCER PATIENTS.

\author{
Bram Ramaekers \\ Manuela Joore \\ Béranger Lueza \\ Julia Bonastre \\ Audrey Mauguen \\ Jean-Pierre Pignon \\ Cecile Le Pechoux \\ Dirk De Ruysscher \\ MAR-LC Collaborative Group \\ Janneke Grutters
}




\section{Abstract}

Objective: To examine and compare the cost-effectiveness of different modified radiotherapy schemes and CRT in the curative treatment of unresected NSCLC patients.

Methods: A probabilistic Markov model was developed based on individual patient data from the Meta-Analysis of Radiotherapy in Lung Cancer (MAR-LC; $\mathrm{N}=2,000$ ). Dutch healthcare costs, quality-adjusted life years (QALYs) and net monetary benefits (NMB) were compared between two accelerated schemes (very accelerated (VART) and moderately accelerated (MART)), two hyperfractionated schemes (using an identical $\left(H R T^{l}\right)$ or higher $\left(H R T^{H}\right)$ total treatment dose than CRT) and CRT.

Results: All modified fractionations were more effective and costly than CRT (1.12 QALYs, €24,360). VART and MART were most effective (1.30 and 1.32 QALYs) and cost $€ 25,746$ and $€ 26,208$ respectively. $\mathrm{HRT}^{\top}$ and $\mathrm{HRT}^{\mathrm{H}}$ yielded less QALYs than the accelerated schemes (1.27 and 1.14 QALYs), and cost $€ 26,199$ and $€ 29,683$ respectively. MART had the highest NMB $(€ 79,322 ; 95 \%$ $\mathrm{Cl} € 35,478-€ 133,648)$ and was the most cost-effective treatment followed by VART $(€ 78,347 ; 95 \% \mathrm{Cl} € 64,635-€ 92,526)$. CRT had a NMB of $€ 65,125(95 \%$ $\mathrm{Cl} € 54,663-€ 75,537)$. MART had the highest probability of being cost-effective (43\%), followed by VART (31\%), HRT' $(24 \%), \mathrm{HRT}^{\mathrm{H}}(2 \%)$ and CRT $(0 \%)$.

Conclusions: Implementing accelerated radiotherapy is almost certainly more efficient than current practice (CRT) and should be recommended as standard radiotherapy for the curative treatment of unresected NSCLC not receiving concurrent chemo-radiotherapy.

Keywords: Radiotherapy; Dose Fractionation; Non-Small-Cell Lung Cancer; Cost-Benefit Analysis; Markov Chain 


\section{Introduction}

Non-small cell lung cancer (NSCLC) comprises $85 \%$ of all lung cancer which is the third most diagnosed form of cancer and causes the greatest number of cancer deaths. ${ }^{1,2}$ Radiotherapy with or without chemotherapy is increasingly being used in the curative treatment for unresected NSCLC. ${ }^{3}$ The therapeutic effect of radiation alone in lung cancer follows a clear dose-response relationship (i.e. higher biological doses lead to better local tumor control). ${ }^{4-6}$ Hence, as recently shown by an individual patient meta-analysis, ${ }^{7}$ modified fractionation radiotherapy schemes, with increased biological dose, have the ability to improve overall survival (OS) compared with conventional radiotherapy schedules (hazard ratio (HR): 0.88). Additionally, modified radiotherapy increased the risk of acute esophageal toxicity. ${ }^{7}$

With regard to the scarcity of resources and accelerating costs of cancer care, it is increasingly important to consider the cost-benefit ratio of (new) treatments to guide decision-making. ${ }^{8,9}$ Economic evaluations are frequently performed using decision-analytic modelling to synthesize different sources of evidence (e.g. effectiveness, patient reported outcomes and costs), compare the costeffectiveness of competing interventions and support decision-making under uncertainty. $^{9}$ As cost-effectiveness estimates are inevitably surrounded by uncertainty, it is essential to characterize uncertainty in economic evaluations. ${ }^{9}$ Although parameter uncertainty (as exact estimates for parameters like effectiveness are often unknown) is frequently acknowledged in decision-analytic modelling, patient heterogeneity is often ignored. ${ }^{10,11}$ The objective of the present study is to perform a cost-effectiveness analysis comparing multiple modified fractionation radiotherapy schemes with conventional fractionation radiotherapy in the curative treatment of unresected NSCLC while taking into account both parameter uncertainty and patient heterogeneity.

\section{Methods}

\section{MAR-LC}

The Meta-Analysis of Radiotherapy in Lung Cancer (MAR-LC) database was used as primary data source. This database consists of individual patient data from twelve randomized clinical trials (RCTs) that compared. conventional and modified fractionated radiotherapy. ${ }^{7}$ The ten RCTs with a population of unresected NSCLC patients were selected for the present study. These RCTs accrued a total of 2,000 patients between 1989 and 2006 (median follow-up: 6.9 years). In four trials, the same chemotherapy was administered in both arms either concomitantly with radiotherapy (two trials) or as induction chemotherapy 
(two trials). No chemotherapy was given in the other RTCs. Most patients were male $(75 \%)$, aged $60-69$ years $(42 \%)$, had squamous cell carcinoma (SCC) $(60 \%)$ and stage III disease (83\%). Performance status was good (Eastern Cooperative Oncology Group (ECOG) performance status $=0$ ) for $43 \%$ of the patients.

\section{Markov model description}

A probabilistic decision-analytic Markov cohort model was developed. To compare competing interventions, this model aims to reflect the course of a disease using a hypothetical cohort of patients who transit between mutually exclusive health states. ${ }^{9}$ These health states were based on whether patients were alive and presence of toxicity (Figure 6.1). Subsequently, the expected costs and effects were estimated for conventional fractionated radiotherapy and four types of modified fractionation radiotherapy. These modified fractionation schemes are based on two types of modified fractionation and their combination: 1) accelerated radiotherapy schemes which consist of a reduced overall treatment time (OTT) compared with conventional fractionation and 2) hyperfractionated radiotherapy schemes which consist of a higher number of fractions with a smaller dose per fraction compared with conventional radiotherapy. Five schemes were compared using the MAR-LC database (Appendix 6.1):

1. Conventional fractionation radiotherapy (CRT; 10 trials, $N=944): 5$ weekly fractions of 1.8-2.0 Gray (Gy), accumulating to a total treatment dose (TTD) of $60-70 \mathrm{~Gy}$.

2. Very accelerated radiotherapy (VART; 6 trials, $N=700$ ): reduced OTT with $\geq 50 \%$, using an identical $( \pm 5 \%)$ or lower $(5-10 \%)$ TTD compared with CRT (OS HR: 0.88 (95\% confidence interval $(95 \% \mathrm{Cl})$ 0.78-0.98) versus CRT). ${ }^{7}$

3. Moderately accelerated radiotherapy (MART; 1 trial, $N=29$ ): reduced OTT with $14-49 \%$, using a TTD identical $( \pm 5 \%)$ to CRT (OS HR: $0.90(95 \% \mathrm{Cl}$ $0.52-1.54$ ) versus $\mathrm{CRT})^{7}$

4. Hyperfractionated radiotherapy using identical TTD (HRT'; 2 trials, $N=164)$ : the average dose per fraction is decreased to $\leq 1.75$ Gy using a TTD identical $( \pm 5 \%)$ to CRT (OS HR: 0.87 (95\% Cl 0.69-1.10) versus CRT). ${ }^{7}$

5. Hyperfractionated radiotherapy using higher TTD $\left(H R T^{H} ; 1\right.$ trial, $\left.N=163\right)$ : the average dose per fraction is decreased to $\leq 1.75$ Gy using a higher $(5-15 \%)$ TTD than CRT (OS HR: 0.92 (95\% Cl 0.74-1.15) versus CRT). ${ }^{7}$

A lifetime time horizon and a cycle time of one month were used. Additionally, a half-cycle correction was applied. Future costs and effects were discounted by rates of $4.0 \%$ and $1.5 \%$ respectively, according to the Dutch pharmacoeconomic guideline. 


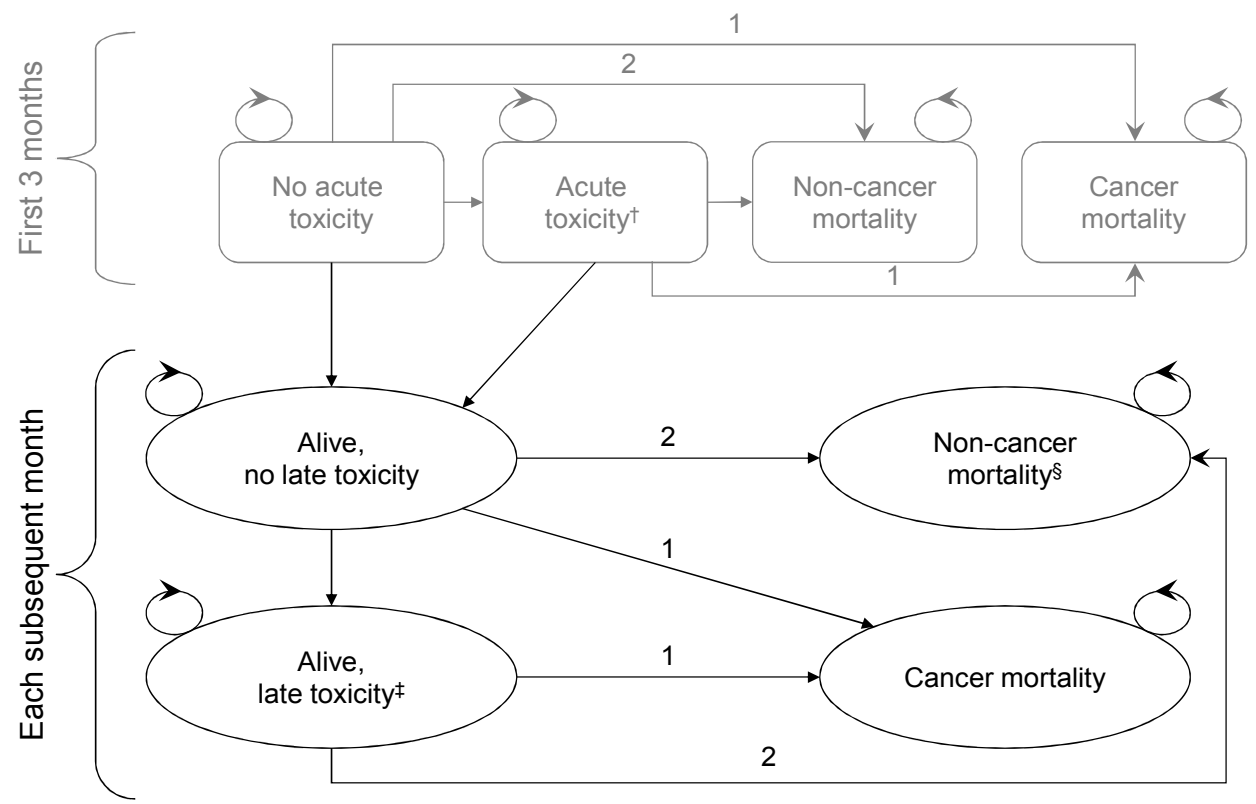

Figure 6.1: Diagrammatical representation of the Markov model structure*

* The numbers ( 1 and 2 ) next to the arrows correspond to the Weibull models in Appendix 6.2.

$\dagger$ Acute toxicity included grade 3 or higher pulmonary toxicity, esophageal toxicity and hematological toxicity (see Appendix 6.3 for the corresponding logistic regression models).

$\ddagger$ Late toxicity included grade 3 or higher pulmonary toxicity and esophageal toxicity (Table 6.1).

$\S$ Non-cancer mortality was defined as deaths resulting from causes other than cancer and not occurring after disease progression.

Since a model is a simplified representation of reality, assumptions about reality are inherent to modelling. The main assumptions were:

1. Based on an analysis of the Radiation Therapy Oncology Group database, acute toxicity was assumed to increase from start of radiotherapy to 3 months and then reverse. ${ }^{12}$ Late toxicity was assumed to be irreversible, to begin 3 months after start of radiotherapy and to increase in frequency up to 1.5 years with the assumption that it had plateaued. ${ }^{12}$

2. There is no overlap between toxicities, i.e. a patient can only have one toxicity and not for example both pulmonary and esophageal toxicity concurrently. However, one patient can have different acute and late toxicities. No overlap between toxicities was assumed since there was only limited overlap in the MAR-LC database ( $3.1 \%$ for acute and $0.1 \%$ for late toxicity). Incorporating overlapping toxicities would unnecessarily increase the complexity and hence decrease the transparency of the model.

3. As mentioned above, modified radiotherapy may reduce non-cancer mortality (most likely due to differences in treatment-related death). ${ }^{7}$ Nevertheless, we conservatively assumed that there is no difference in non-cancer mortality between the radiotherapy schemes. This was assumed since non- 
cancer mortality, which included treatment-related deaths, was not reported for MART.

\section{Transition probabilities}

Time dependent survival probabilities were estimated by means of parametric survival models using a Weibull distribution (Appendix 6.2). ${ }^{13}$ Separate Weibull regression models were developed for non-cancer mortality and cancer mortality. Logistic regression models were developed to estimate acute toxicity (grade 23) probabilities, separately for acute pulmonary, esophageal and hematological toxicity (Appendix 6.3). All regression models were stratified by trial to preserve randomization and obtain unbiased estimates. ${ }^{14}$ Potential heterogeneity in baseline risks was acknowledged through these regression models using the following covariates: treatment arm (CRT; VART; MART; HRT'; HRT ${ }^{\mathrm{H}}$ ), sex (male; female), age ( $\leq 59 ; 60-69 ; \geq 70)$, performance status (mild; good), histology (SCC; non-SCC) and disease stage (I/II; IIIA; IIIB). All variables were included in the initial model as categorical variables. Selection of covariates was performed as described by Hosmer et al, ${ }^{15}$ except for the treatment arm variable. The treatment arm variable was not included in the Weibull model to predict non-cancer mortality, and was always included in the Weibull regression model that predicts cancer mortality. Individual characteristics were needed to calculate the acute toxicity probabilities using the logistic regression models. For this purpose, a hypothetical cohort of individual patients was replicated based on average characteristics and their correlations from the MAR-LC database (Appendix 6.3).

To estimate late pulmonary and esophageal toxicity (grade $\geq 3$ ), the proportions from the MAR-LC database were used. Consistent with the meta-analysis, late toxicity was assumed to be equal for all comparators (Table 6.1). ${ }^{7}$

All parameters retrieved from the MAR-LC database were computed in the Department of Biostatistics and Epidemiology of IGR by BL and BR.

\section{Effects and costs}

Utility scores were used as effect measure. Utility is a single score measure for generic health-related quality of life and ranges from 0 (death) to 1 (full health). These utility scores were combined with life expectancy to calculate quality adjusted life years (QALYs). Utility scores were derived from a Dutch crosssectional study $(\mathrm{N}=260)^{16}$ which used the Euroqol-5D ${ }^{17}$ questionnaire. Patients with unresected NSCLC $(\mathrm{N}=85)$ were selected from this study (Table 6.1).

Patients who died due to NSCLC in the Markov model were assigned a disutility. This was the average disutility for recurrent disease (0.152) multiplied by the average life expectancy after recurrent disease $(6$ months in the MAR-LC database). 
Costs were calculated using the Dutch health care perspective and converted to the 2011 price level based on price indices from Statistics Netherlands (CBS). Resource use and unit prices are reported in Table 6.2.

Table 6.1: Input parameters: probabilities and health state utility

\begin{tabular}{|c|c|c|c|c|c|}
\hline \multicolumn{2}{|l|}{ Parameter } & $\begin{array}{l}\text { Estimated } \\
\text { value }\end{array}$ & $\begin{array}{l}\text { Standard } \\
\text { error }\end{array}$ & Distribution & Source \\
\hline \multicolumn{6}{|l|}{ Probabilities } \\
\hline \multicolumn{2}{|c|}{ Probability of non-cancer mortality } & \multicolumn{4}{|c|}{ Dependent on time and patient characteristics (Appendix 6.2) } \\
\hline \multicolumn{2}{|c|}{ Probability of cancer mortality } & \multicolumn{4}{|c|}{ Dependent on time and patient characteristics (Appendix 6.2) } \\
\hline \multicolumn{2}{|c|}{ Probability of acute pulmonary toxicity } & \multicolumn{4}{|c|}{ Dependent on patient characteristics (Appendix 6.3) } \\
\hline \multicolumn{2}{|c|}{ Probability of acute esophageal toxicity } & \multicolumn{4}{|c|}{ Dependent on patient characteristics (Appendix 6.3) } \\
\hline \multicolumn{2}{|c|}{ Probability of acute hematological toxicity } & \multicolumn{4}{|c|}{ Dependent on patient characteristics (Appendix 6.3) } \\
\hline \multicolumn{2}{|c|}{ Probability of late pulmonary toxicity* } & $15.4 \%$ & $14.0 \%$ & Beta & MAR-LC \\
\hline \multicolumn{2}{|c|}{ Probability of late esophageal toxicity ${ }^{*}$} & $3.3 \%$ & $2.9 \%$ & Beta & MAR-LC \\
\hline \multicolumn{6}{|c|}{ Health state utility } \\
\hline \multirow[t]{6}{*}{ No recurrence } & No toxicity & 0.800 & 0.029 & Beta & 16 \\
\hline & Acute hematological toxicity ${ }^{\dagger}$ & 0.710 & & & 16,18 \\
\hline & Acute pulmonary toxicity $\ddagger$ & 0.493 & 0.075 & Beta & 16 \\
\hline & Acute esophageal toxicity & 0.493 & 0.075 & Beta & 16 \\
\hline & Late pulmonary toxicity $\ddagger$ & 0.493 & 0.075 & Beta & 16 \\
\hline & Late esophageal toxicity $\ddagger$ & 0.493 & 0.075 & Beta & 16 \\
\hline \multirow[t]{6}{*}{ Recurrence } & No toxicity & 0.794 & 0.038 & Beta & 16 \\
\hline & Acute hematological toxicity ${ }^{\dagger}$ & 0.704 & & & 16,18 \\
\hline & Acute pulmonary toxicity $\ddagger$ & 0.129 & 0.061 & Beta & 16 \\
\hline & Acute esophageal toxicity $\ddagger$ & 0.129 & 0.061 & Beta & 16 \\
\hline & Late pulmonary toxicity $\ddagger$ & 0.129 & 0.061 & Beta & 16 \\
\hline & Late esophageal toxicity ${ }^{\ddagger}$ & 0.129 & 0.061 & Beta & 16 \\
\hline
\end{tabular}

Abbreviations: NSCLC $=$ non-small cell lung cancer

* It was assumed that late toxicity increases from 3 months after start radiotherapy up to 18 months after start radiotherapy to the total probability. The monthly probability was then calculated from the total probability using the following formula: ${ }^{9}$

$\mathrm{P}(1$ month $)=1-\mathrm{e}^{(\ln (1-P(\text { total })) * 1 / 15)}$

† This health state utility was calculated based the utilities without toxicity from Grutters et al., ${ }^{16}$ and a disutility from Nafees et al., ${ }^{18}$

¥ It was assumed that patients with acute or late esophageal or pulmonary toxicity had the same utility scores. 
Table 6.2: Input parameters: resource use and costs

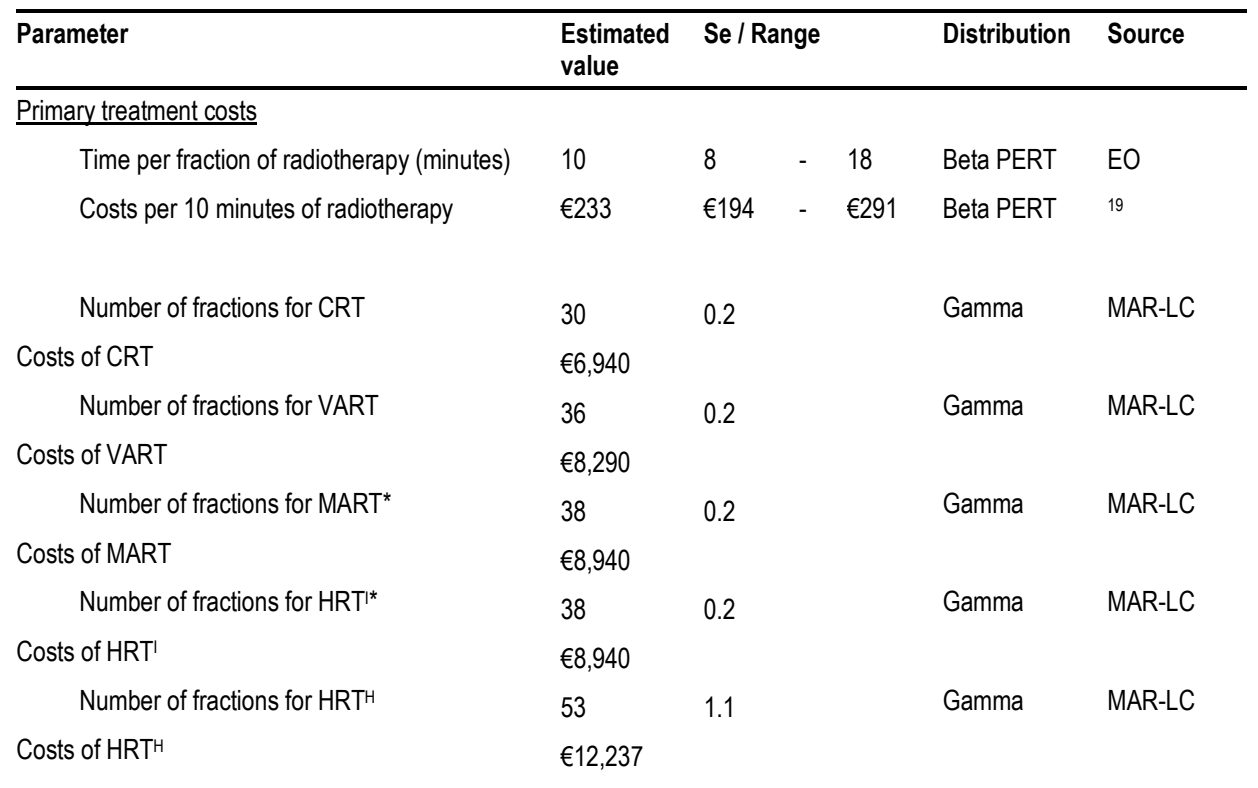

Acute pulmonary toxicity costs ( $\geq$ grade 3 )

Probability of hospitalization (\%)
Days of hospital admission
Costs Hospital admission (per day)
Medication costs

Acute pulmonary toxicity costs

$\begin{array}{llll}2.5 & 0.3 & \text { Beta } & \text { EO } \\ 11 & 2.0 & \text { Gamma } & 20 \\ € 463 & \text { Fixed } & & 21 \\ € 22 & \text { Fixed } & & \text { CVZ }\end{array}$

$\underline{\text { Acute esophageal toxicity costs ( } \geq \text { grade } 3 \text { ) }}$

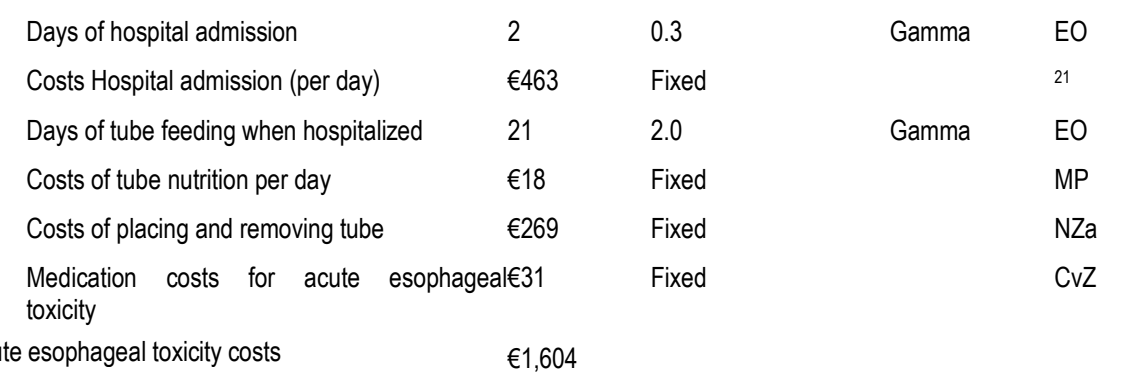

Abbreviations: $\mathrm{Se}=$ standard error, $\mathrm{EO}=$ expert opinion, $\mathrm{MP}=$ market price, $\mathrm{NZa}=$ Nederlands Zorgautoriteit $/$ Dutch Healthcare Authority, CvZ = College voor Zorgverzekeringen $/$ Health Care Insurance Board

* Due to a lack of data, the number of fractions was assumed to be $96 \%$ of the theoretical number of fractions (as observed on average for the other comparators) and the standard error from very accelerated radiotherapy was used

† Standard error was based on expert opinion

$\ddagger$ The chemotherapy costs have to be multiplied by the proportion of patients who received chemotherapy $(29.5 \%)$ to calculate the average chemotherapy costs 
Table 6.2 (continued): Input parameters: resource use and costs

\begin{tabular}{|c|c|c|c|c|}
\hline Parameter & $\begin{array}{l}\text { Estimated } \\
\text { value }\end{array}$ & Se / Range & Distribution & Source \\
\hline \multicolumn{5}{|l|}{ Acute hematological toxicity costs ( $\geq$ grade 3 ) } \\
\hline Costs of an episode of febrile neutropenia & $€ 3,754$ & $€ 1241$ & Gamma & 22 \\
\hline \multicolumn{5}{|c|}{ Mortality costs (costs of last life-year before dying) } \\
\hline Cancer mortality & $€ 22,793$ & $€ 2000^{\dagger}$ & Gamma & 23 \\
\hline Non-cancer mortality & $€ 16,246$ & $€ 2000^{\dagger}$ & Gamma & 23 \\
\hline
\end{tabular}

Follow-up costs

Costs per follow-up visit
Number of follow-up visits in first year

$€ 73 \quad$ Fixed

$4 \quad$ Fixed

NSCLC guideline

Monthly costs of follow-up first year

$€ 24$

Number of follow-up visits in second year

2

Fixed

NSCLC

guideline

Monthly costs of follow-up second year

$€ 12$

Number of follow-up visits after second year

Fixed

NSCLC

guideline

Monthly costs of follow-up after second year

$€ 6$

$\underline{\text { Late Toxicity costs }}$

Yearly costs of irreversible dyspnoea $\geq$ grade $3 € 1099$

$€ 100+$

20

Monthly costs of irreversible dyspnoea $\geq$ grade $3 € 92$

Abbreviations: $\mathrm{Se}=$ standard error, $\mathrm{EO}=$ expert opinion, $\mathrm{MP}=$ market price, $\mathrm{NZa}=$ Nederlandse Zorgautoriteit / Dutch Healthcare Authority, CvZ = College voor Zorgverzekeringen / Health Care Insurance Board

* Due to a lack of data, the number of fractions was assumed to be $96 \%$ of the theoretical number of fractions (as observed on average for the other comparators) and the standard error from very accelerated radiotherapy was used

† Standard error was based on expert opinion

‡ The chemotherapy costs have to be multiplied by the proportion of patients who received chemotherapy $(29.5 \%)$ to calculate the average chemotherapy costs

\section{Markov model analysis}

Expected life years (LYs), QALYs, costs and net monetary benefit (NMB) were estimated for all comparators. The NMB was calculated by multiplying the number of QALYs with the ceiling ratio and subtracting the total costs. The treatment strategy with the highest NMB is considered as most cost-effective.

We adopted a ceiling ratio of $€ 80,000$, since this is the informal ceiling ratio for a high burden of disease in the Netherlands. ${ }^{18}$ The incremental cost-effectiveness ratio (ICER) was calculated by dividing the incremental costs by the incremental QALYs. The ICER represents the costs of an additional QALY gained and was used to estimate the cost-effectiveness of a treatment 1) opposed to CRT and 
2) opposed to the next best alternative. A treatment is deemed cost-effective when its ICER is below the ceiling ratio.

The Markov model was analysed in Microsoft Excel 2003 (computer software, Microsoft Corporation, Redmond, United States). The required analyses to retrieve the input parameters were performed in SAS version 9.2 (computer software, SAS Institute, Cary, United States), except the Weibull analysis which was performed in $\mathrm{R}$ version 2.13.1 (open source computer software, $\mathrm{R}$ Foundation, Vienna, Austria) as SAS did not support stratifying by trial in this analysis.

\section{Parameter uncertainty}

To explore the impact of parameter uncertainty on the estimated (cost)effectiveness, probabilistic sensitivity analysis was performed using Monte Carlo simulation $\left(15,000\right.$ iterations). ${ }^{9}$ For this purpose a distribution was assigned to the input parameters (Table 6.1 and 6.2). The Weibull and logistic regression models were included using Cholesky decompositions. ${ }^{9}$ Costeffectiveness acceptability curves were created to show for different ceiling ratios the probability that a treatment is most cost-effective. ${ }^{9}$

Since the estimated cost-effectiveness is surrounded by uncertainty it is possible that based on current information, the 'wrong' decision is being made. The expected value of perfect information (EVPI) analysis quantifies the costs of this decision uncertainty. It estimates the value of further research to gain knowledge of the 'true' parameter values. ${ }^{9}$ Thus, the EVPI represents the upper limit that society should be willing to pay to reduce decision uncertainty and inform the decision in the future. ${ }^{9}$ The EVPI per patient was multiplied by the effective population in the next five years (expected lifespan of the technology) and discounted by a rate of $4 \%$ to calculate the population EVPI. The effective population was calculated based on a yearly incidence of 8,661 NSCLC patients in the Netherlands (Dutch Cancer Registration, 2010) minus the estimated proportion of resected NSCLC patients $(20 \%)$ and the estimated proportion with metastatic disease among unresected patients $(40 \%)$, this resulted in an annual population of 4,157 patients. To identify the most valuable research topics, the EVPI for (groups of) parameters (EVPPI) was calculated.

\section{Heterogeneity}

The expected value of individualized care (EVIC) was calculated to examine the impact of patient heterogeneity on cost-effectiveness. ${ }^{19,20}$ The EVIC estimates the value of providing the optimal treatment for each individual instead of the average best treatment for all patients. The same hypothetical cohort of individual patients as for the logistic regressions was used for this calculation (Appendix 6.3). The EVIC per patient was estimated by calculating 1) the NMB of the optimal treatment per patient $\left.\left(\mathrm{NMB}_{\text {patient_max }}\right) ; 2\right)$ the $\mathrm{NMB}$ of the average best treatment $\left(\mathrm{NMB}_{\text {average_max }}\right)$; 3) $\mathrm{EVIC}_{\text {patient }}=\mathrm{NMB}_{\text {patient_max }}-\mathrm{NMB}_{\text {average_max }}$ for all 
individual patients and 4) calculating the average $\mathrm{EVIC}_{\text {patient }}$ and multiplying it by the effective population.

\section{Results}

Expected survival ranged from 20 months for CRT $(1.63 \mathrm{LYs})$ and $\mathrm{HRT}^{\mathrm{H}}(1.66$ LYs), to 22 months for HRT' (1.83 LYs), up to 23 months for VART (1.88 LYs) and MART (1.90 LYs). MART was also the most effective treatment (1.32 QALYs) in terms of QALYs, followed by VART (1.30 QALYs), HRT ${ }^{\prime}$ (1.27 QALYs), $\mathrm{HRT}^{\mathrm{H}}$ (1.14 QALYs) and CRT (1.12 QALYs). HRT ${ }^{\mathrm{H}}$ was most costly (€29,683), followed by MART (€26,208), HRT' $(€ 26,199)$, VART $(€ 25,746)$ and CRT $(€ 24,360)$. Costs differences were mainly due to differences in the number of fractions leading to differences in primary treatment costs.

CRT was both the least effective and least expensive treatment. Compared with CRT, all comparators except HRT ${ }^{\mathrm{H}}$ (ICER: $\left.€ 228,852\right)$ were cost-effective with ICERs ranging between $€ 7,592$ (VART) and $€ 12,379\left(\mathrm{HRT}^{\prime}\right)$.

$\mathrm{HRT}^{\mathrm{H}}$ was both more effective $(0.02 \mathrm{QALYs})$ and expensive $(€ 5,323)$ than CRT. This resulted in an ICER of $€ 228,852$. Given the ceiling ratio of $€ 80,000$ per QALY gained, $H R T^{H}$ was not cost-effective opposed to CRT. HRT' was also more effective $(0.15$ QALYs) and expensive $(€ 1,839)$ than CRT leading to an ICER below the ceiling ratio $(€ 12,379)$. HRT $T^{\top}$ was thus cost-effective opposed to CRT. VART was more effective (0.03 QALYs) and less expensive (€453) and thus dominated HRT'. MART was more effective (0.02 QALYs) and more expensive (€462) than VART. The calculated ICER $(€ 25,716)$ was below the ceiling ratio. Thus, MART is the most cost-effective radiotherapy scheme (Table 6.3).

Figure 6.2 and Appendix 6.4 show the uncertainty surrounding the results. Taking into account this uncertainty, MART had the highest probability of being cost-effective $(43 \%)$, followed by VART $(31 \%), \mathrm{HRT}^{\top}(24 \%), \mathrm{HRT}^{\mathrm{H}}(2 \%)$ and CRT (0\%) (Figure 6.2). Additionally, the estimated EVPI was €228 million (Figure 6.3). More specifically, the EVPPI indicated that further research would be most valuable for the primary treatment costs of MART ( $€ 8.2$ million) and cancer mortality after VART ( $€ 6.7$ million) and MART ( $€ 5.4$ million). The EVIC showed a value of individualizing care of $€ 0.1$ million (Figure 6.3). 


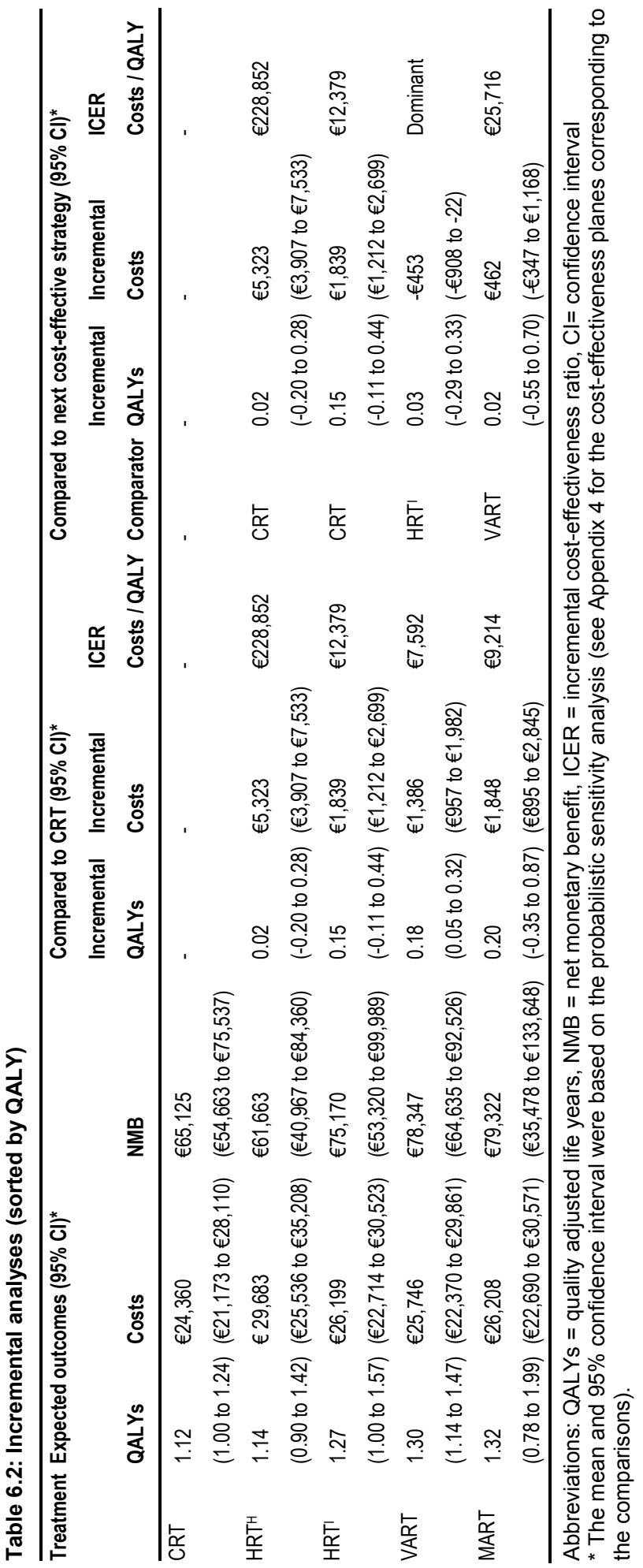




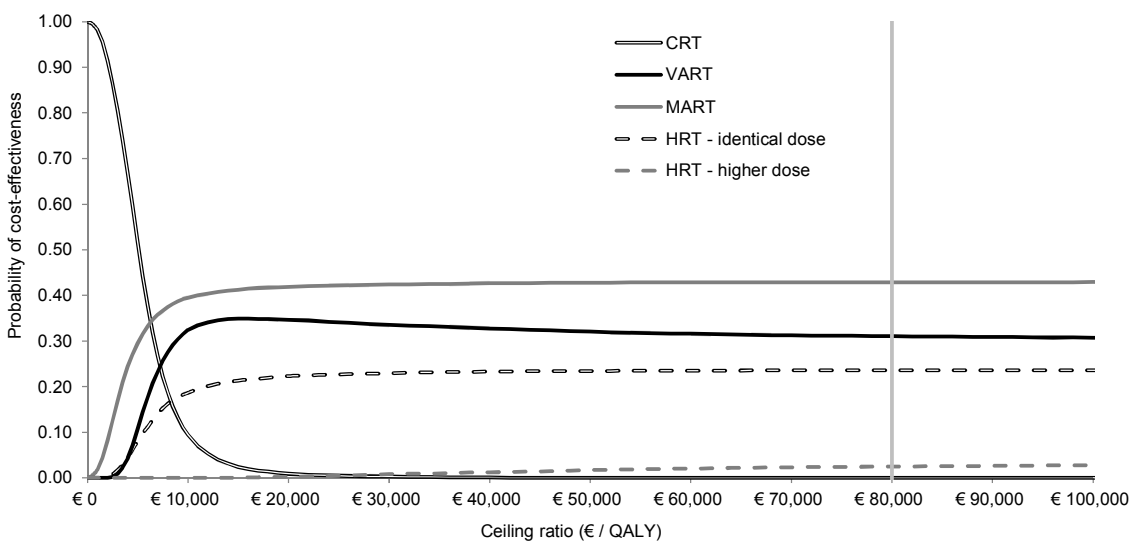

Figure 6.2: Cost-effectiveness acceptability curves*

* The vertical line represents the ceiling ratio which was adopted in our analyses (€80,000 per QALY gained).

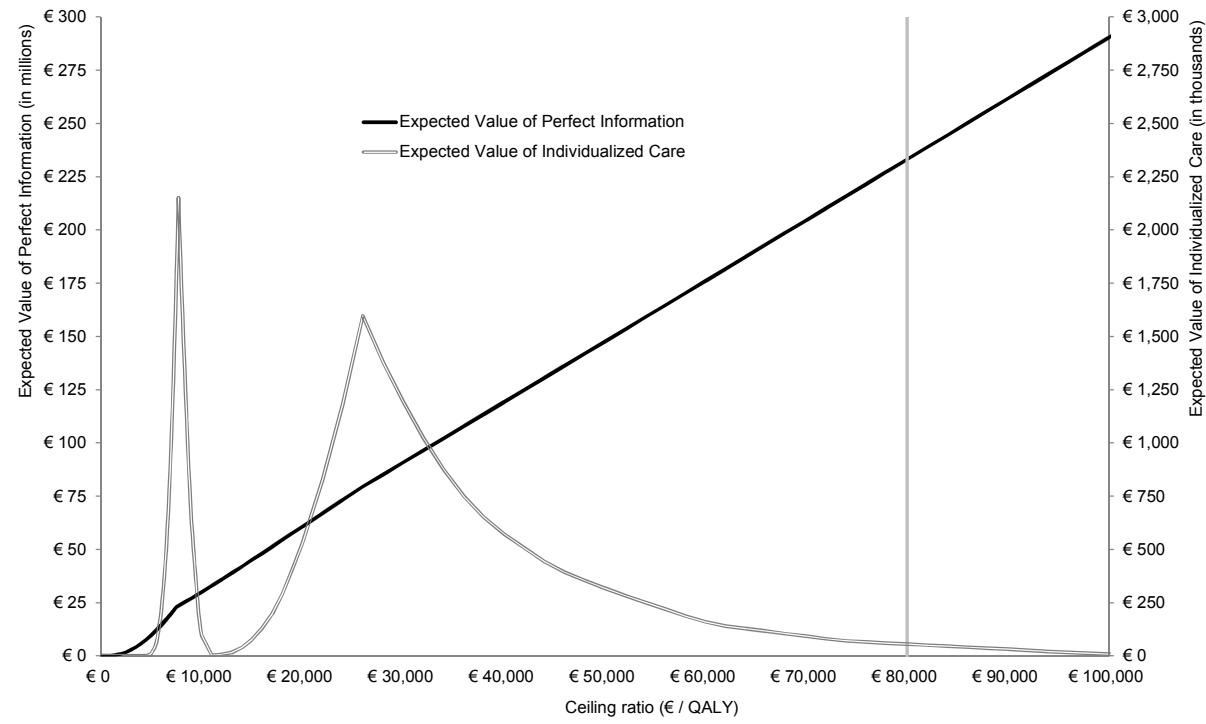

Figure 6.3: Expected value of individualized care and expected value of perfect information*

* The vertical line represents the ceiling ratio which was adopted in our analyses (€80,000 per QALY gained).

\section{Discussion}

All modified radiotherapy schemes were more effective and more costly than CRT. While MART was the most cost-effective treatment strategy, the differences between comparators were small, and all confidence intervals for the incremental costs and effects were overlapping (Table 6.3). Therefore, it is 
uncertain which modified fractionation strategy is most cost-effective. Moreover, estimated survival after MART was based on only one study with a small number of patients $(\mathrm{N}=58)$. Despite this uncertainty, modified fractionation radiotherapy in general is likely (>99\%) cost-effective compared with CRT and accelerated schemes are likely to be the most effective and cost-effective modified fractionation schemes. However, it is unclear which accelerated fractionation scheme is deemed optimal. The comparison of MART versus VART resulted in a $51 \%$ probability for MART and $49 \%$ probability for VART of being costeffective (Appendix 6.4). Additionally, in the individual patient meta-analysis, heterogeneity in the relative treatment effect between the different radiotherapy schemes was not demonstrated. ${ }^{7}$ In this paper we examined patient heterogeneity based on differences in baseline risk, and found that there was relatively little value to individualize care (i.e. to provide different treatments to different patients). Instead, it would be more valuable to perform further research to reduce parameter uncertainty, specifically for primary treatment costs of MART and cancer mortality after VART and MART.

Our study was the first to assess the cost-effectiveness of several modified radiotherapy schemes in NSCLC. In one prior analysis, also using a Markov model, an ICER of $€ 11,576$ was estimated for continuous hyperfractionation accelerated radiotherapy (CHART) compared to CRT; concluding that CHART is likely cost-effective in Belgium. ${ }^{21}$ This CHART trial was included in the VART arm in our study. The comparison in our study of VART and CRT would result in a slightly more beneficial ICER for VART of $€ 7,592$.

The present study limitations were, firstly, that the health care perspective was used instead of the societal perspective. Therefore, productivity losses at work were not incorporated. Since $48 \%$ of the study population was at treatment start above the Dutch pensionable age, no large differences between the perspectives are expected. Secondly, all MAR-LC trials compare modified radiotherapy and CRT (Appendix 6.1). Hence, the comparisons between different modified fractionated radiotherapy schemes are based on indirect evidence. Although synthesis of head-to-head comparisons of RCTs provides the most valid evidence of treatment effectiveness, it has been recommended to consider indirect treatment comparisons if direct evidence is unavailable. ${ }^{22,23}$ The comparisons were stratified by trial, comparing patients only within each trial (preserving randomization), to obtain unbiased estimates. ${ }^{14}$ Thirdly, concomitant chemoradiotherapy is the current standard while this was administered in two out of the ten included trials. Only one study used cisplatin-doublet chemotherapy during radiotherapy (NCCTG 94242), which is at present considered to be the standard concurrent schedule. We were therefore unable to examine the impact of concomitant chemo-radiotherapy. Nevertheless, compared with CRT the benefit of modified radiotherapy with chemotherapy on overall survival $(\mathrm{HR}=0.92,95 \% \mathrm{Cl} 0.77-1.10$ ) was not significantly (interaction $\mathrm{p}=0.57$ ) lower than for modified radiotherapy without chemotherapy $(\mathrm{HR}=0.87,95 \% \mathrm{Cl} 0.78-$ 
0.97). ${ }^{7}$ Additionally, although this was not observed in a recent phase II study, ${ }^{24}$ modified radiotherapy delivered concurrently with chemotherapy may increase acute side effects opposed to concurrent chemotherapy with conventional radiotherapy. In case of increased toxicity when providing concomitant chemoradiotherapy, the incremental costs for modified radiotherapy compared with CRT might on the one hand increase due to potentially increased toxicity management costs. On the other hand, it might result in lower incremental costs for instance due to interrupting and stopping both chemotherapy and radiotherapy (before finishing all chemotherapy cycles and radiotherapy fractions). Thus, if chemotherapy was added to all included trials, the incremental effects are expected to be similar and the impact on incremental costs is unclear. This issue should be addressed in future (economic) studies. Fourthly, to avoid unnecessary complexity in the model, it was decided not to incorporate overlapping toxicities (as described in the model description section). Despite this simplification of the model, the total occurrence of the acute and late toxicities in the present model would be equal to a more complex model incorporating 'overlap' between toxicities. Also, as described in the methods section, overlap between available toxicities in the MAR-LC database was small. Therefore, considering this low proportion of overlap between toxicities and the equivalence between total occurrence of toxicity, this model assumption is unlikely to have a large impact on the study results. Finally, the applied survival of six months after recurrence, to calculate the disutility for cancer mortality, could be criticized in view of the 10-12 months median survival in newly diagnosed stage IV NSCLC. ${ }^{25}$ Nevertheless, this was a conservative assumption, i.e. longer survival after recurrence and thus a higher disutility, will favor the modified radiotherapy treatments since these have less NSCLC deaths (and thus fewer disutilities).

It is likely that with recent advancements in radiotherapy techniques, the same level of acceleration can be given safely in fewer fractions, e.g. 24 fractions instead of 38 fractions (as for VART and MART). ${ }^{26,27}$ This could reduce the costs (due to the lower number of fractions) while maintaining the survival benefit of accelerated radiotherapy. $\mathrm{HRT}^{\prime}$, the third most cost-effective option in our analysis, applies split-course radiotherapy. Although this was not observed in the individual patient meta-analysis, ${ }^{7}$ it is widely believed to be less efficient than continued radiotherapy schemes. As a result, split-course radiotherapy is rarely used nowadays. ${ }^{28}$ Also, concurrent chemotherapy and CRT is nowadays the treatment of choice for good performance patients with locally advanced NSCLC. ${ }^{29}$ However, as many patients are not eligible for concomitant chemoradiotherapy treatment, ${ }^{30}$ sequential chemotherapy and accelerated radiotherapy seems a promising treatment option. In the present analysis we were unable to examine the role of chemotherapy in combination with modified fractionation radiotherapy. Although the benefit of modified radiotherapy with and without chemotherapy did not differ significantly, ${ }^{7}$ the results of RTOG 9410 and RTOG 
0617 caution against assuming that modifying conventional concomitant chemo-radiotherapy will improve the therapeutic ratio. ${ }^{31,32}$ For sequential chemotherapy and radiotherapy, there are no indications that there would be an interaction between both modalities for toxicity. Hence, the present study results are probably most applicable to patients not receiving concomitant chemoradiotherapy. Studies examining the role of modified fractionation radiotherapy combined with concomitant chemotherapy are warranted. This includes examining for instance whether it is safe to provide chemotherapy concurrently with accelerated radiotherapy, whether the benefits of modified fractionation are preserved in case of concurrent chemo-radiotherapy and whether other wellstudied $^{27}$ types of accelerated once-daily high-dose radiotherapy are costeffective.

In conclusion, it remains uncertain which modified scheme is most cost-effective and it is unclear whether the study results can be extrapolated to modified radiotherapy combined with concomitant chemotherapy. Hence, further research comparing the cost-effectiveness of different types of modified radiotherapy and examining the role of chemotherapy might be valuable. Nevertheless, implementing accelerated radiotherapy is almost certainly more costeffective than current practice (CRT) for patients treated with sequential chemoradiotherapy or radiotherapy alone. Hence, waiting for more evidence before implementing accelerated radiotherapy (without concomitant chemotherapy) would lead to health benefits forgone. In addition, if future evidence would show that accelerated radiotherapy is not the most cost-effective radiotherapy type, the forgone implementation costs (sunk costs) are expected to be low. Therefore, despite available uncertainty, it is encouraged to adopt accelerated radiotherapy for the curative treatment of unresected NSCLC patients who do not receive concurrent chemo-radiotherapy and examine its role in the context of concurrent chemo-radiotherapy.

\section{Acknowledgement}

We greatly acknowledge the following research groups who agreed to share their data: Arbeitsgemeinschaft Radioonkologie der Deutschen Krebsgesellschaft, Eastern Cooperative Oncology Group, Medical Research Council, North Central Cancer Treatment Group, and Radiation Therapy Oncology Group. The contents of this publication and methods used are solely the responsibility of the authors.

This study was supported by an unrestricted research grant (No. 152002021) from the Dutch Organization of Health Research and Development (ZonMw) and the French National League against Cancer. ZonMw and the French National League against Cancer had no influence on the study design, data analyses, data interpretation, manuscript writing or the decision to submit the manuscript for publication. 


\section{References}

1. Ferlay J, Shin HR, Bray F, et al: Cancer Incidence and Mortality Worldwide: IARC CancerBase No. 10 Accessed on Cited 2012 June 20th. http://globocan.iarc.fr

2. Siegel R, Naishadham D, Jemal A: Cancer statistics, 2012. CA Cancer J Clin 62:10-29, 2012

3. Blackstock AW, Govindan R: Definitive chemoradiation for the treatment of locally advanced non small-cell lung cancer. J Clin Oncol 25:4146-52, 2007

4. Belderbos JS, Heemsbergen WD, De Jaeger K, et al: Final results of a Phase I/II dose escalation trial in non-small-cell lung cancer using three-dimensional conformal radiotherapy. Int $\mathrm{J}$ Radiat Oncol Biol Phys 66:126-34, 2006

5. Kong FM, Ten Haken RK, Schipper MJ, et al: High-dose radiation improved local tumor control and overall survival in patients with inoperable/unresectable non-small-cell lung cancer: longterm results of a radiation dose escalation study. Int J Radiat Oncol Biol Phys 63:324-33, 2005

6. Willner J, Baier K, Caragiani E, et al: Dose, volume, and tumor control prediction in primary radiotherapy of non-small-cell lung cancer. Int J Radiat Oncol Biol Phys 52:382-9, 2002

7. Mauguen A, Le Péchoux C, Saunders MI, et al: Hyperfractionated or accelerated radiotherapy in lung cancer: an individual patient data meta-analysis. J Clin Oncol 30:2788-97, 2012

8. Meropol NJ, Schrag D, Smith TJ, et al: American Society of Clinical Oncology guidance statement: the cost of cancer care. J Clin Oncol 27:3868-74, 2009

9. Briggs A, Sculpher MJ, Claxton K: Decision Modelling for Health Economic Evaluation. Oxford, Oxford University Press, 2006

10. Grutters JP, Sculpher M, Briggs AH, et al: Acknowledging patient heterogeneity in economic evaluation: a systematic literature review. PharmacoEconomics 31:111-123, 2013

11. Jain R, Grabner M, Onukwugha E: Sensitivity analysis in cost-effectiveness studies: from guidelines to practice. Pharmacoeconomics 29:297-314, 2011

12. Werner-Wasik M, Paulus R, Curran WJ, Jr., et al: Acute esophagitis and late lung toxicity in concurrent chemoradiotherapy trials in patients with locally advanced non-small-cell lung cancer: analysis of the radiation therapy oncology group (RTOG) database. Clin Lung Cancer 12:245-51, 2011

13. Carroll KJ: On the use and utility of the Weibull model in the analysis of survival data. Control Clin Trials 24:682-701, 2003

14. Buyse $M$, Piedbois $P$, Piedbois $Y$, et al: Meta-analysis: methods, strengths, and weaknesses. Oncology (Williston Park) 14:437-43; discussion 444, 447, 2000

15. Hosmer DW, Lemeshow S, May S: Applied Survival Analysis: Regression Modeling of Time to Event Data (ed 2), John Wiley \& Sons, 2008 pp. 416

16. Grutters JPC, Joore MA, Wiegman EM, et al: Health-related quality of life in patients surviving non-small cell lung cancer. Thorax 65:903-7, 2010

17. EuroQol Group: EuroQol--a new facility for the measurement of health-related quality of life. The EuroQol Group. Health Policy 16:199-208, 1990

18. Council for Public Health and Health Care. Sensible and sustainable care [in Dutch]. Zoetermeer: Council for Public Health and Health Care, 2006.

19. Basu A, Meltzer D: Value of information on preference heterogeneity and individualized care. Med Decis Making 27:112-27, 2007

20. Van Gestel A, Grutters J, Schouten J, et al: The role of the expected value of individualized care in cost-effectiveness analyses and decision making. Value Health 15:13-21, 2012

21. Lievens $\mathrm{Y}$, Kesteloot $\mathrm{K}$, Van den Bogaert $\mathrm{W}$ : CHART in lung cancer: economic evaluation and incentives for implementation. Radiother Oncol 75:171-8, 2005

22. Bucher HC, Guyatt GH, Griffith LE, et al: The results of direct and indirect treatment comparisons in meta-analysis of randomized controlled trials. J Clin Epidemiol 50:683-91, 1997

23. National Institute for Health and Clinical Excellence. Guide to the methods of technology appraisal. London: National Institute for Health and Clinical Excellence 2008. 
24. Van Baardwijk A, Reymen B, Wanders S, et al: Mature results of a phase II trial on individualised accelerated radiotherapy based on normal tissue constraints in concurrent chemoradiation for stage III non-small cell lung cancer. Eur J Cancer 48:2339-46, 2012

25. Paz-Ares LG, Biesma B, Heigener D, et al: Phase III, Randomized, Double-Blind, PlaceboControlled Trial of Gemcitabine/Cisplatin Alone or With Sorafenib for the First-Line Treatment of Advanced, Nonsquamous Non-Small-Cell Lung Cancer. J Clin Oncol, 2012

26. Van Elmpt W, De Ruysscher D, van der Salm A, et al: The PET-boost randomised phase II dose-escalation trial in non-small cell lung cancer. Radiother Oncol 104:67-71, 2012

27. Belderbos J, Uitterhoeve L, van Zandwijk N, et al: Randomised trial of sequential versus concurrent chemo-radiotherapy in patients with inoperable non-small cell lung cancer (EORTC 08972-22973). Eur J Cancer 43:114-21, 2007

28. Dubben $\mathrm{HH}$, Krull A, Beck-Bornholdt HP: Split-course radiotherapy: where do we stand? Strahlenther Onkol 177:227-39, 2001

29. Auperin A, Le Pechoux C, Rolland E, et al: Meta-analysis of concomitant versus sequential radiochemotherapy in locally advanced non-small-cell lung cancer. J Clin Oncol 28:2181-90, 2010

30. De Ruysscher D, Botterweck A, Dirx M, et al: Eligibility for concurrent chemotherapy and radiotherapy of locally advanced lung cancer patients: a prospective, population-based study. Ann Oncol 20:98-102, 2009

31. Curran WJ, Jr., Paulus R, Langer CJ, et al: Sequential vs. concurrent chemoradiation for stage III non-small cell lung cancer: randomized phase III trial RTOG 9410. J Natl Cancer Inst 103:1452-60, 2011

32. Bradley JD, Paulus R, Komaki R, et al: A randomized phase III comparison of standard-dose (60 Gy) versus high-dose (74 Gy) conformal chemoradiotherapy +/- cetuximab for stage IIIA/IIIB non-small cell lung cancer: preliminary findings on radiation dose in RTOG 0617, American Society for Radiation Oncology (ASTRO). Miami Beach, 2011 


\section{Appendix 6.1: Classification of MAR-LC trials}

10 trials $(\mathrm{N}=2000)$

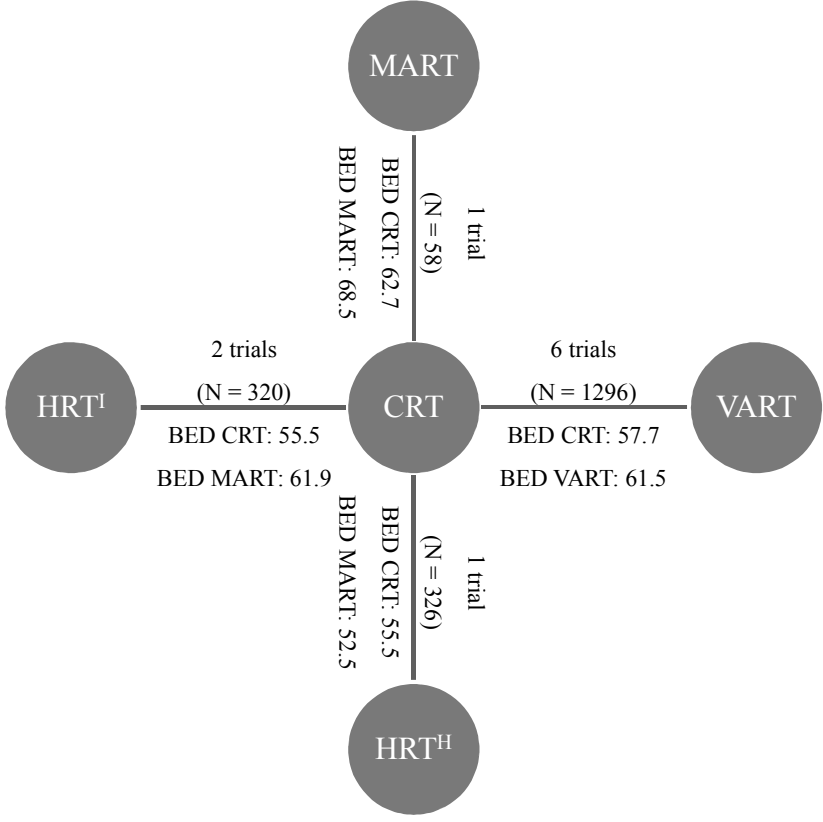

Abbreviation: $\mathrm{BED}=$ biologic effective dose (average in case of multiple trials) 


\section{Appendix 6.2: Input parameters for survival}

The Weibull models were constructed according to the intention-to-treat principle using the following covariates: treatment arm (CRT; VART; MART; HRT'; $\mathrm{HRT}^{\mathrm{H}}$ ), sex (male; female), age ( $\left.\leq 59 ; 60-69 ; \geq 70\right)$, performance status (mild; good), histology (SCC; non-SCC) and disease stage (I/II; IIIA; IIIB). All variables were included in the initial model as categorical variables. Selection of covariates was performed as described by Hosmer et al, ${ }^{1}$ except for the treatment arm variable. This variable was not included in the equation to predict noncancer mortality, and was always included in the model for cancer mortality. The parameterization of the Weibull model is as follows:

1) $\quad S(t)=e^{-\lambda t^{\alpha}}$

Where $S(t)=$ survival probability at time $t$. The shape parameter $(\alpha)$ could be retrieved from the analysis output. Lambda $(\lambda)$ the event rate parameter was calculated by the sum of all coefficients multiplied by the accompanying covariates $(\mathrm{X})$ :

2) $\lambda=\beta_{\text {Intercept }}+\beta_{\text {Treament arm }} X_{\text {Treatment arm }}+\beta_{\text {Age 60-69 }} X_{\text {Age 60-69 }}+\beta_{\text {Age 70+ }} X_{\text {Age 70+ }}$

$+\beta_{\text {Female }} X_{\text {Female }}+\beta_{\text {Performance status good }} X_{\text {Performance status good }}$

$+\beta_{\text {Histology Squamous cell }} X_{\text {Histology Squamous cell }}+\beta_{\text {Disease Stage IIIA }} X_{\text {Disease Stage IIIA }}$

$+\beta_{\text {Disease Stage IIIB }} X_{\text {Disease Stage IIIB }}$

The coefficients $(\beta)$ for all covariates were retrieved from the analysis output. The coefficient for the intercept was calculated based on the shape $(\alpha)$ and scale parameters from the $R$ output:

3) $\quad \beta_{\text {Intercept }}=-\operatorname{Ln}($ scale $) \times$ shape

One essential statistical technique when analyzing multiple trials is stratification by trial, which guarantees that patients are compared within each trial and not across trials. ${ }^{2}$ The Weibull model was stratified by trial, which resulted in separate scale and shape parameters for each trial. The different scale and shape parameters were pooled using a random effects model. ${ }^{3}$ Subsequently, all coefficients were multiplied by the accompanying average covariates to calculate $\lambda$. For instance the proportion of female patients $X_{\text {Female }}$ was multiplied by the coefficient for female $ß_{\text {Female }}$. The time-dependent transition probability between two cycles (between $t_{1}$ and $t_{2}$ ), was then calculated using the following formula (derived from equation 1 ):

4)

$$
S\left(t_{2}-t_{1}\right)=e^{-\lambda\left(\mathrm{t}_{1}{ }^{\alpha}-\mathrm{t}_{2}{ }^{\alpha}\right)}
$$




\section{Estimated regression coefficients for survival probabilities}

\begin{tabular}{|c|c|c|c|c|}
\hline \multirow[t]{2}{*}{ Parameter } & \multicolumn{2}{|c|}{$\begin{array}{l}\text { Equation 1: probability of cancer } \\
\text { mortality (1644 events) }\end{array}$} & \multicolumn{2}{|c|}{$\begin{array}{l}\text { Equation 2: probability of non-cancer } \\
\text { mortality ( } 205 \text { events) }\end{array}$} \\
\hline & Estimated value & Se & Estimated value & Se \\
\hline \multicolumn{5}{|c|}{ Model characteristics } \\
\hline Model distribution & Weibull $^{*}$ & & Weibull $^{*}$ & \\
\hline Shape (a) & 1.093 & 0.200 & 0.920 & 0.372 \\
\hline $\operatorname{Ln}($ scale) & 6.635 & 0.053 & 8.389 & 0.147 \\
\hline Intercept ${ }^{\dagger}$ & -7.252 & & -7.722 & \\
\hline \multicolumn{5}{|c|}{ Explanatory baseline characteristics§ } \\
\hline \multicolumn{5}{|l|}{ Treatment arm } \\
\hline VART & -0.176 & 0.064 & & \\
\hline MART & -0.169 & 0.276 & & \\
\hline HRT' & -0.137 & 0.128 & & \\
\hline $\mathrm{HRT}^{\mathrm{H}}$ & -0.022 & 0.119 & & \\
\hline \multicolumn{5}{|l|}{ Age (year) } \\
\hline $60-69$ & -0.123 & 0.061 & 0.378 & 0.219 \\
\hline $70+$ & -0.149 & 0.070 & 0.688 & 0.223 \\
\hline \multicolumn{5}{|l|}{ Sex } \\
\hline Female & -0.149 & 0.059 & -0.372 & 0.188 \\
\hline \multicolumn{5}{|l|}{ Performance status } \\
\hline Good & -0.237 & 0.053 & -0.508 & 0.160 \\
\hline \multicolumn{5}{|l|}{ Disease stage } \\
\hline IIIA & 0.242 & 0.081 & & \\
\hline IIIB & 0.384 & 0.083 & & \\
\hline \multicolumn{5}{|c|}{ Mean $2 Y$ probability $\ddagger$} \\
\hline CRT & $62 \%$ & & $18 \%$ & \\
\hline VART & $56 \%$ & & $18 \%$ & \\
\hline MART & $56 \%$ & & $18 \%$ & \\
\hline HRT' & $57 \%$ & & $18 \%$ & \\
\hline HRTH & $61 \%$ & & $18 \%$ & \\
\hline Source & MAR-LC & & MAR-LC & \\
\hline
\end{tabular}

Abbreviation: $\mathrm{Se}=$ standard error, $2 \mathrm{Y}=2$-year

* Included in the probabilistic sensitivity analysis using a multivariate normal distribution which was constructed using Cholesky decompositions (multivariate normal distribution) ${ }^{4}$

†Calculated using the following formula: $-\mathrm{Ln}($ scale $) \times$ shape

$\ddagger$ This probability represents the mean 2-year probability for the separate Weibull models (not the 2year probability as in the Markov trace)

$\S$ Histology was excluded (according to the purposeful selection of covariates algorithm by Hosmer and Lemeshow). ${ }^{1,5}$ 


\section{Appendix 6.3: Input parameters for acute toxicity}

The included covariates and subsequent selection procedure were the same for acute pulmonary and esophageal toxicity as described for the Weibull models (Appendix 6.2). This was also the case for hematological toxicity, except that the treatment arm was excluded as covariate since it is caused by chemotherapy and independent of radiation fractionation scheme. The parameterization of the logistic model is as follows:

$$
P=\frac{e^{z}}{1+e^{z}}
$$

Where $P$ is the toxicity probability and $z$ was calculated by the sum of all coefficients multiplied by the accompanying covariates $(X)$ :

2) $\quad z=\beta_{\text {Intercept }}+\beta_{\text {Treatment arm }} X_{\text {Treatment arm }}+\beta_{\text {Age 60-69 }} X_{\text {Age 60-69 }}+\beta_{\text {Age 70+ }} X_{\text {Age 70+ }}$

$+\beta_{\text {Female }} X_{\text {Female }}+\beta_{\text {Performance status good }} X_{\text {Performance status good }}$

$+\beta_{\text {Histology Squamous cell }} X_{\text {Histology Squamous cell }}+\beta_{\text {Disease Stage IIIA }} X_{\text {Disease Stage IIIA }}$

$+\beta_{\text {Disease Stage IIIB }} X_{\text {Disease Stage IIIB }}$

As for the Weibull models, the logistic regression models were stratified by trial. However, no coefficient for the intercept is given if the logistic regression models are stratified by trial in SAS, thus absolute toxicity probabilities based could not be calculated based on this logistic regression model. Therefore, separate logistic regression models were constructed for each trial using the covariates as selected in the above described logistic regression model stratified by trial. The obtained coefficients for each trial were pooled using a random effects model. ${ }^{3}$ To calculate the acute toxicity probabilities using the logistic regression models, individual characteristics were needed. For this purpose, a hypothetical cohort of individual patients with individual characteristics was replicated based on the average characteristics and their correlations from the MAR-LCdatabase. For each patient, the individual $z$ values and toxicity probabilities were calculated. To obtain the toxicity probabilities for the whole cohort, the individual probabilities were averaged. This was done separately for each comparator. 
Estimated regression coefficients for acute toxicity ( $\geq$ grade 3 ) probabilities*

\begin{tabular}{|c|c|c|c|c|c|c|}
\hline \multirow[t]{2}{*}{ Parameter } & \multicolumn{2}{|c|}{$\begin{array}{l}\text { Equation 1: probability } \\
\text { of acute pulmonary toxicity } \\
\text { (77 events) }{ }^{\dagger}\end{array}$} & \multicolumn{2}{|c|}{$\begin{array}{l}\text { Equation 2: probability } \\
\text { of acute esophageal toxicity } \\
\text { (304 events) }\end{array}$} & \multicolumn{2}{|c|}{$\begin{array}{l}\text { Equation 3: probability } \\
\text { of acute hematological } \\
\text { toxicity (202 events) }\end{array}$} \\
\hline & $\begin{array}{l}\text { Estimated } \\
\text { value }\end{array}$ & Se & $\begin{array}{l}\text { Estimated } \\
\text { value }\end{array}$ & Se & $\begin{array}{l}\text { Estimated } \\
\text { value }\end{array}$ & Se \\
\hline \multicolumn{7}{|c|}{ Explanatory baseline characteristics ${ }^{\ddagger}$} \\
\hline Intercept & -2.856 & 0.273 & -2.429 & 0.268 & -1.892 & 1.418 \\
\hline \multicolumn{7}{|l|}{ Trial arm } \\
\hline VART & -0.625 & 0.324 & 1.281 & 0.216 & & \\
\hline MART/HRTH & 0.086 & 0.405 & 0.428 & 0.539 & & \\
\hline $\mathrm{HRT}^{\prime}$ & 0.016 & 0.839 & -0.157 & 0.314 & & \\
\hline \multicolumn{7}{|l|}{ Age (year) } \\
\hline 60-69 & 0.360 & 0.323 & & & 0.364 & 0.560 \\
\hline $70+$ & 0.737 & 0.354 & & & 1.033 & 0.374 \\
\hline \multicolumn{7}{|l|}{ Sex } \\
\hline Female & & & 0.672 & 0.204 & 0.963 & 0.313 \\
\hline \multicolumn{7}{|c|}{ Mean probability§ } \\
\hline CRT & $7.8 \%$ & & $9.8 \%$ & & $24.7 \% \|$ & \\
\hline VART & $4.4 \%$ & & $27.8 \%$ & & $24.7 \% \|$ & \\
\hline MART/HRTH & $8.5 \%$ & & $14.3 \%$ & & $24.7 \% \|$ & \\
\hline $\mathrm{HRT}^{\prime}$ & $8.0 \%$ & & $8.6 \%$ & & $24.7 \% \|$ & \\
\hline Source & MAR-LC & & MAR-LC & & MAR-LC & \\
\hline
\end{tabular}

Abbreviation: $\mathrm{Se}=$ standard error

* Included in the probabilistic sensitivity analysis using a multivariate normal distribution which was constructed using Cholesky decompositions(multivariate normal distribution). ${ }^{4}$

$\dagger$ To handle the occurrence of zero events in $2 \times 2$ tables between dependent and independent variables (leading to quasi-complete separation), the Firth's penalized maximum likelihood estimation method ${ }^{6,7}$ was used for four logistic regression models.

$\ddagger \mathrm{A}$ combined estimate was calculated for $\mathrm{HRT}^{\mathrm{H}}$ and MART. This was done because acute toxicity was not reported in the MART trial and the overall treatment time and total treatment dose are similar for these two comparators In addition, performance status, histology and disease stage were excluded (according to the purposeful selection of covariates algorithm by Hosmer and Lemeshow). ${ }^{1,5}$

§It was assumed that acute toxicity increased from start radiotherapy to 3 months thereafter to the total probability (reported in the table). The monthly probability was then calculated from the total probability using the following formula: ${ }^{4}$

$P(1$ month $)=1-e^{\left(\ln (1-P(\text { total }))^{*}+1 / 3\right)}$

II Hematological toxicity is mainly caused by the administration of chemotherapy rather than the radiotherapy treatment scheme and was therefore assumed to be independent of the radiotherapy scheme (and thus equal for all comparators). The calculated probability $(24.7 \%)$ was conditional on that patients received chemotherapy and has to be multiplied by the proportion of patients who received chemotherapy (29.5\%; assumed equal among all comparators) to calculate the average probability of acute hematological toxicity per comparator $(7.3 \%)$. 


\section{Appendix 6.4: Cost-effectiveness planes corresponding to the} comparisons in Table 6.3

$H R T^{H}$ versus $C R T^{*}$

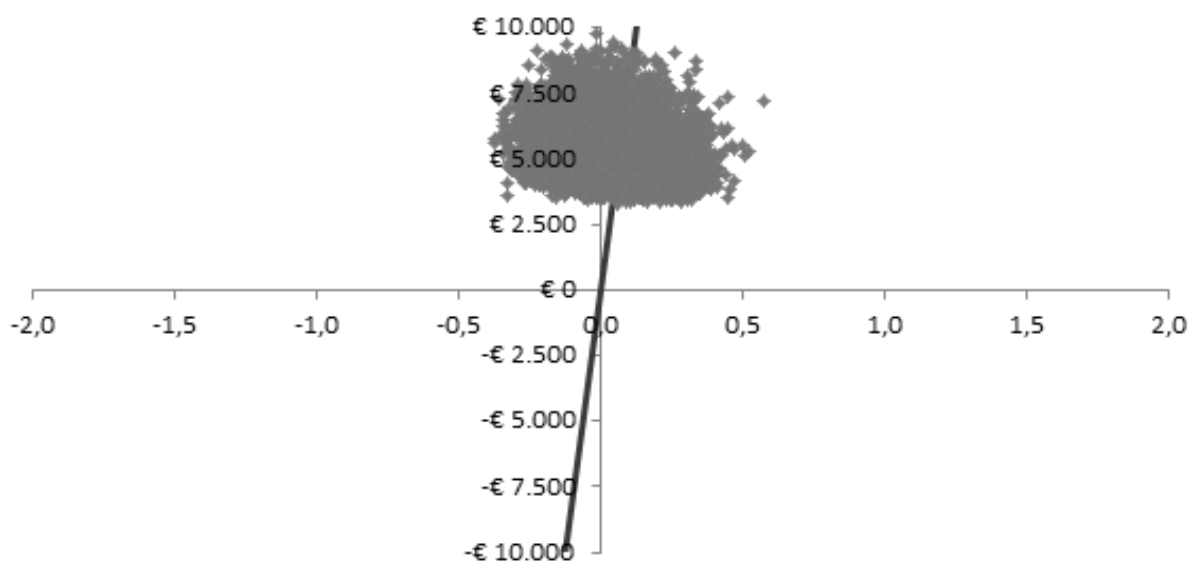

$H R T^{\prime}$ versus $C R T^{*}$

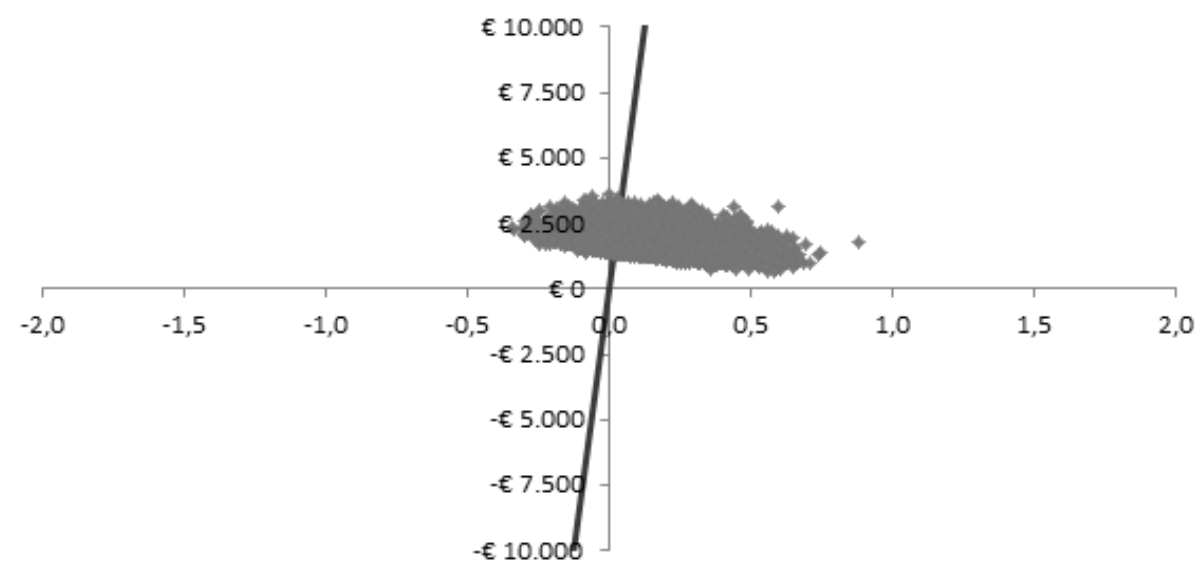

\begin{tabular}{|c|c|c|c|}
\hline HRTH versus CRT & $\%$ simulations & $\overline{H R T '}$ versus CRT & $\%$ simulations \\
\hline North-West quadrant & $44.0 \%$ & North-West quadrant & $14.5 \%$ \\
\hline North-East quadrant & $56.0 \%$ & North-East quadrant & $85.5 \%$ \\
\hline South-West quadrant & $0.0 \%$ & South-West quadrant & $0.0 \%$ \\
\hline South-East quadrant & $0.0 \%$ & South-East quadrant & $0.0 \%$ \\
\hline HRT ${ }^{H}$ cost-effective & $35.2 \%$ & HRT' cost-effective & $80.7 \%$ \\
\hline CRT cost-effective & $64.8 \%$ & CRT cost-effective & $19.3 \%$ \\
\hline
\end{tabular}

* The diagonal line represents the ceiling ratio which was adopted in our analyses $(€ 80,000$ per QALY gained). 
VART versus $C R T^{*}$

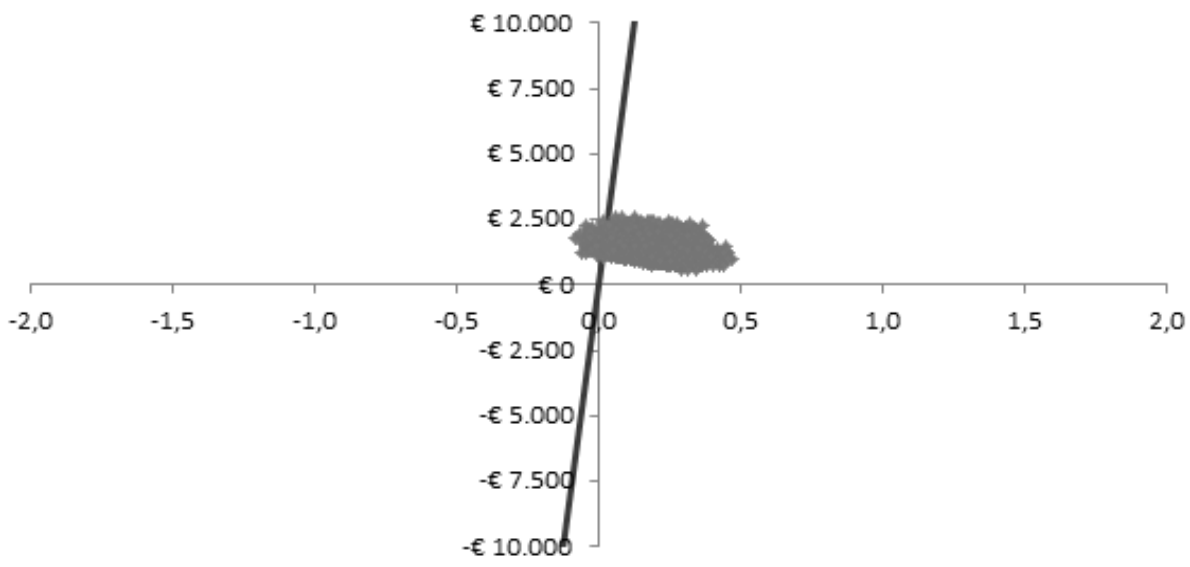

\section{MART versus $C R T^{*}$}

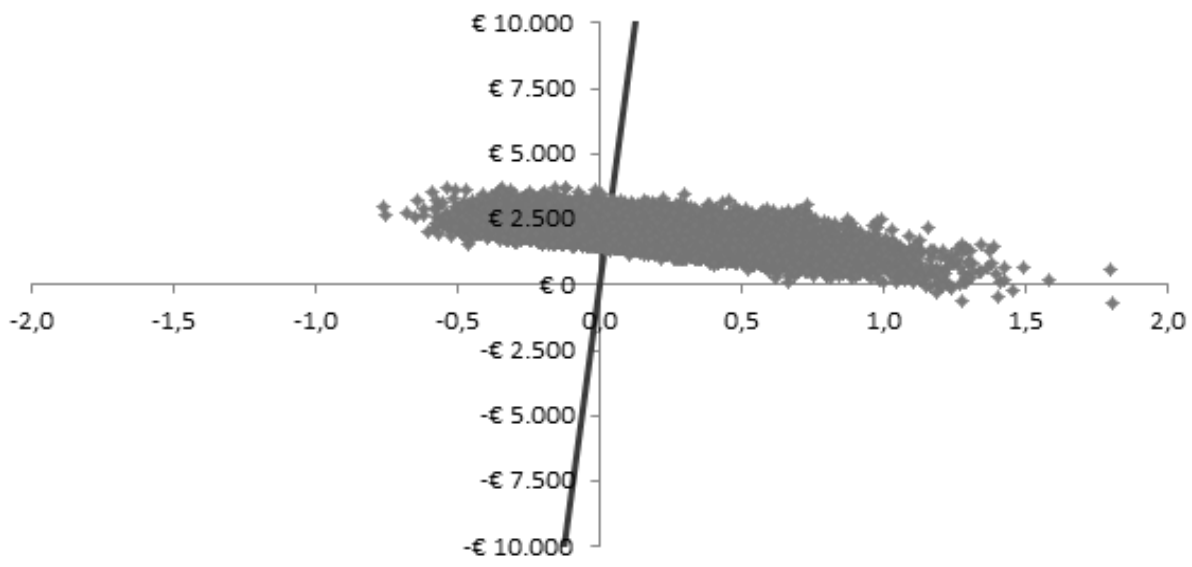

\begin{tabular}{ll}
\hline VART versus CRT & $\%$ simulations \\
\hline North-West quadrant & $0.4 \%$ \\
North-East quadrant & $99.6 \%$ \\
South-West quadrant & $0.0 \%$ \\
South-East quadrant & $0.0 \%$ \\
& \\
VART cost-effective & $99.1 \%$ \\
CRT cost-effective & $0.9 \%$ \\
\hline
\end{tabular}

\begin{tabular}{ll}
\hline MART versus CRT & $\%$ simulations \\
\hline North-West quadrant & $27.3 \%$ \\
North-East quadrant & $82.6 \%$ \\
South-West quadrant & $0.0 \%$ \\
South-East quadrant & $0.1 \%$ \\
& \\
MART cost-effective & $69.7 \%$ \\
CRT cost-effective & $30.3 \%$ \\
\hline
\end{tabular}

* The diagonal line represents the ceiling ratio which was adopted in our analyses (€80,000 per QALY gained). 


\section{VART versus $H R T^{*}$}

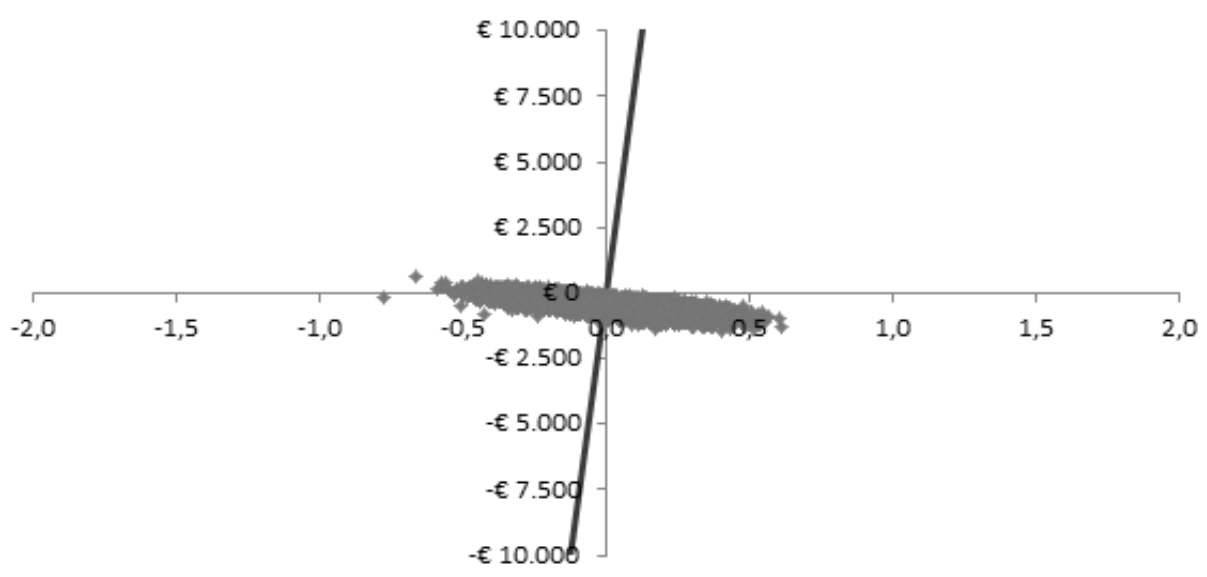

\section{MART versus VART*}

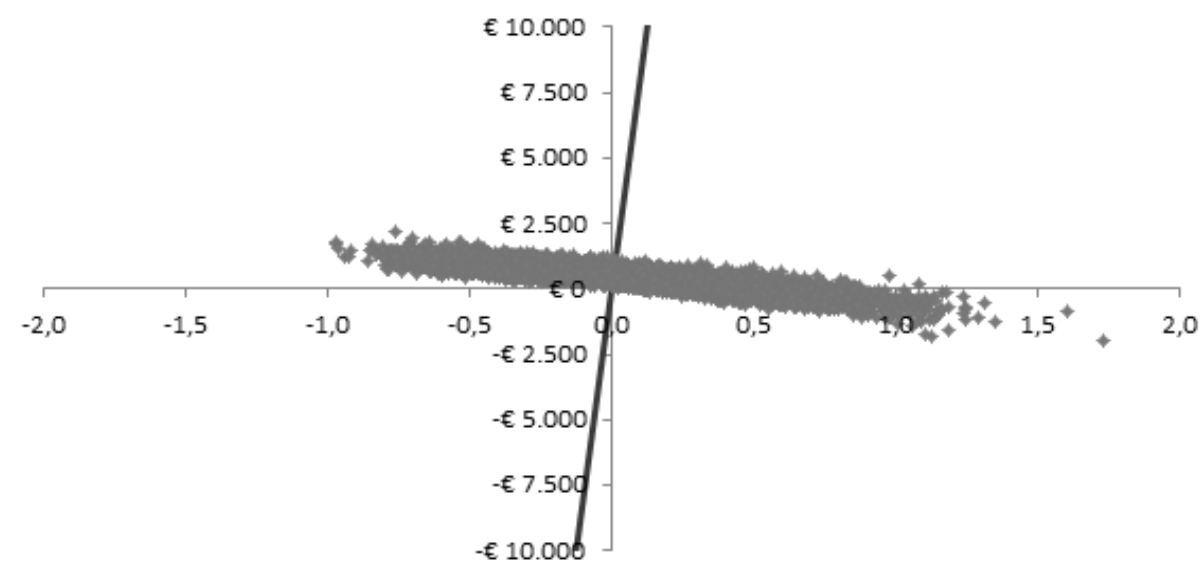

\begin{tabular}{ll}
\hline VART versus HRTI & $\%$ simulations \\
\hline North-West quadrant & $2.1 \%$ \\
North-East quadrant & $0.0 \%$ \\
South-West quadrant & $38.5 \%$ \\
South-East quadrant & $59.4 \%$ \\
& \\
VART cost-effective & $60.6 \%$ \\
HRTI cost-effective & $39.4 \%$ \\
\hline
\end{tabular}

\begin{tabular}{ll}
\hline MART versus VART & $\%$ simulations \\
\hline North-West quadrant & $50.3 \%$ \\
North-East quadrant & $39.0 \%$ \\
South-West quadrant & $0.0 \%$ \\
South-East quadrant & $10.7 \%$ \\
& \\
MART cost-effective & $51.0 \%$ \\
VART cost-effective & $49.0 \%$
\end{tabular}

* The diagonal line represents the ceiling ratio which was adopted in our analyses $(€ 80,000$ per QALY gained). 


\section{Appendix references}

1. Hosmer DW, Lemeshow S, May S: Applied Survival Analysis: Regression Modeling of Time to Event Data (ed 2), John Wiley \& Sons, 2008 pp. 416

2. Buyse $M$, Piedbois $P$, Piedbois $Y$, et al: Meta-analysis: methods, strengths, and weaknesses. Oncology (Williston Park) 14:437-43; discussion 444, 447, 2000

3. Whitehead A: Meta-analysis of Controlled Clinical Trials, John Wiley \& Sons, 2002

4. Briggs A, Sculpher MJ, Claxton K: Decision Modelling for Health Economic Evaluation. Oxford, Oxford University Press, 2006

5. Bursac Z, Gauss $\mathrm{CH}$, Williams DK, et al: Purposeful selection of variables in logistic regression. Source Code Biol Med 3:17, 2008

6. Firth D: Bias Reduction of Maximum Likelihood Estimates. Biometrika 80:27-38, 1993

7. Allison PD: Statistics and Data Analysis Convergence Failures in Logistic Regression. Presented at the SAS Global Forum 2008, Texas, 2008 



\section{PART III}

Discussion

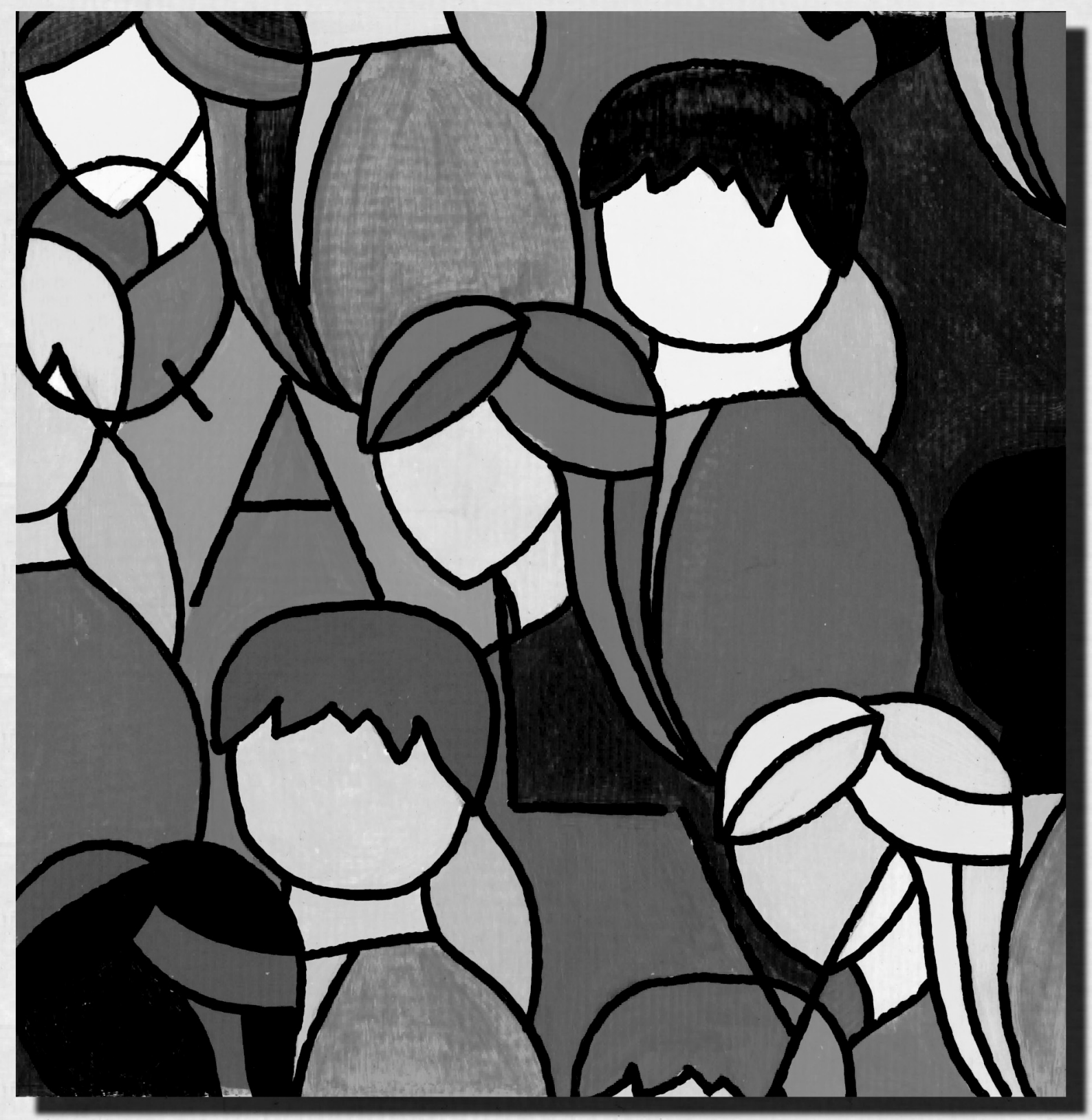





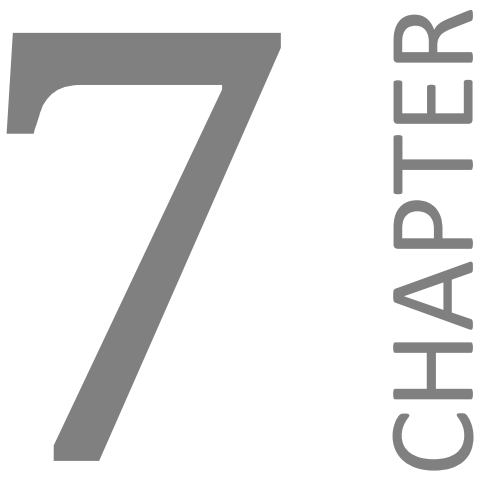

GENERAL DISCUSSION 
CHAPTER 7 
The overall aim of this dissertation, as introduced in Chapter 1, was twofold: 1) to examine recommendations in pharmacoeconomic guidelines on acknowledging patient heterogeneity in economic evaluations (Part I) and; 2) to explore innovative methodology to acknowledge patient heterogeneity in economic evaluations in two case studies; in case comparative studies are lacking and in case individual patient data are available (Part II). This chapter summarizes the main findings, presents methodological considerations, implications for policy, implications for clinical practice and finally this chapter ends with concluding remarks and future perspectives.

\section{Summary of main findings}

In Chapter 2 a systematic review was presented considering guidance from national pharmacoeconomic guidelines on handling patient heterogeneity in economic evaluations. In total, 26 pharmacoeconomic guidelines were included of which 20 advised to identify patient heterogeneity. With regards to the first aim of this thesis, it was concluded based on this review that, although the importance of acknowledging patient heterogeneity in economic evaluation was internationally recognized, it was less clear when and how patient heterogeneity should be acknowledged. This includes which inputs or sources are considered relevant to acknowledge in economic evaluations, whether stratified analysis should be prespecified and how subgroups should be defined. Finally, it was unclear how the results should be presented to inform individualized reimbursement decisions. Based on these findings, we recommend that future pharmacoeconomic guidelines provide specific guidance on the identification of patient heterogeneity, methods to acknowledge patient heterogeneity and presenting the results. The presented checklist may help guideline authorities to formulate their guidance. This could facilitate the systematic and transparent handling of patient heterogeneity in economic evaluations worldwide.

The subsequent three chapters considered the first case study that compared proton and photon radiotherapy for head and neck cancer patients. The aim of this case study was to acknowledge patient heterogeneity in an economic evaluation in case comparative studies are lacking (aim 2a). Chapter 3 synthesized and compared available evidence considering the effectiveness of carbon-ion, proton and photon radiotherapy for head and neck cancer. For this purpose, a systematic review and meta-analyses were performed. In total, 86 observational studies (74 photon, 5 carbon-ion and 7 proton) and eight comparative in-silico studies were included. Since the overall quantity and quality of data regarding carbon-ion and proton therapy was poor and considering the risk of bias in comparisons between (small) observational studies, no definite conclusions were drawn. Despite the scarcity of data, it was suggested that a 
clinically relevant benefit of proton therapy in patients with head and neck cancer probably lies in reducing treatment toxicity. To achieve definite conclusions in the future, it was recommend to construct an international particle therapy register to facilitate definitive comparisons on both survival and treatment toxicity.

Chapter 4 presented an analysis of the impact of late treatment-related xerostomia and dysphagia on generic health-related quality of life (HRQOL) in head and neck cancer patients after radiotherapy. A multi-center cross-sectional survey $(\mathrm{N}=396)$ was performed. It was found that both xerostomia and dysphagia diminished HRQOL substantially, with dysphagia having the strongest impact on HRQOL. This finding emphasizes that next to the expected length of survival, also treatment toxicity and its impact on HRQOL should be considered when treatment choices are made. The results presented in this chapter can be used to assist this trade-off between length and quality of survival. In consideration of this trade-off, xerostomia was more prevalent than dysphagia, whereas dysphagia has a higher impact on HRQOL than xerostomia. Therefore, preventing xerostomia could benefit more patients, whereas preventing dysphagia might result in a larger benefit per patient.

The results of the two previous chapters were used in Chapter 5 to perform a model-based economic evaluation to explore the effectiveness and costeffectiveness of proton radiotherapy compared with radiotherapy with photons for head and neck cancer patients. Proton radiotherapy is a promising radiotherapy modality with a superior dose distribution compared with radiotherapy with photons. However, proton radiotherapy is also associated with higher costs than photon radiotherapy, which are mainly irrecoverable investment costs. As described above, comparative effectiveness research was sparsely available for proton radiotherapy in head and neck cancer. This made it challenging to examine its effectiveness and cost-effectiveness as well as to acknowledge patient heterogeneity in this economic evaluation. To bridge this gap of evidence, normal tissue complication probability (NTCP) models were combined with comparative planning studies in an integrated model. NTCP models estimate the probability of toxicity based on the expected radiation dose to healthy tissues. Comparative planning studies compare the dose-distributions in patients for different radiotherapy techniques. Hence, NTCP models and comparative planning studies can be used in economic evaluations to estimate the expected benefit of innovative radiotherapy techniques for individual patients. This methodology was explored in the presented economic evaluation to handle the lack of comparative effectiveness research and acknowledge patient heterogeneity. It was concluded that proton radiotherapy was on average not cost-effective. However, when considering individual cost-effectiveness estimates, it became clear that proton radiotherapy was potentially worthwhile for individually selected patients. Thus, in this case it was considered valuable to acknowledge patient heterogeneity and examine on the individual level for which patients 
proton radiotherapy is cost-effective and for which patients it is not. Furthermore, value of information analysis indicated that further research to obtain more data on utility scores after xerostomia and NTCP models to predict xerostomia and/or dysphagia was most valuable. Also, it would be valuable to explore which radiotherapy technique makes the most optimal use of the dosimetric advantages of proton therapy. For instance, the comparative planning studies used in the present analysis primarily focused on reducing the radiation dose to the swallowing structures. ${ }^{1}$ It might be of interest to examine the costeffectiveness based on comparative planning studies primarily focused on reducing the radiation dose to parotid glands to reduce the occurrence of xerostomia. Additionally, tumor control probability (TCP) models can be employed to consider strategies wherein the total dose to the tumor is increased to improve the probability of tumor control.

The economic evaluation for the second case study (aim 2b) was presented in Chapter 6. This economic evaluation assessed the cost-effectiveness of modified fractionation radiotherapy as opposed to conventional fractionation radiotherapy in the curative treatment of unresected non-small cell lung cancer patients. In contrast with the previous chapter, where comparative evidence was lacking, individual patient data from 10 randomized controlled trials were available for this case study. Based on available evidence, it was found that accelerated radiotherapy was on average the most cost-effective treatment strategy for all patients. The expected value of individualized care (EVIC) indicated that, in this case, individualized treatment allocation is probably not worthwhile. Thus accelerated radiotherapy was the most cost-effective strategy for all patients and it seemed not valuable to provide individualized treatments with regard to type of radiotherapy fractionation scheme. However, these results are probably most applicable to patients not receiving concomitant chemoradiotherapy as concomitant chemo-radiotherapy is provided in only two of the ten trials and it is unclear whether the benefits of modified radiotherapy are preserved when provided concurrently with chemotherapy. Also, the differences between treatments in terms of effectiveness were small and it was uncertain which type of accelerated radiotherapy scheme was most cost-effective. Nevertheless, implementing any accelerated radiotherapy was almost certainly more efficient than current practice. Therefore, waiting for more evidence before implementing accelerated radiotherapy would lead to health benefits forgone. In addition, if future evidence would show that accelerated radiotherapy is not the most cost-effective radiotherapy type, the forgone implementation costs (sunk costs) are expected to be low. Therefore, it was encouraged to adopt accelerated radiotherapy as standard treatment for the curative treatment of unresected non-small cell lung cancer patients not receiving concomitant chemoradiotherapy. Furthermore, value of information analysis indicated that research to obtain more data on the primary treatment costs for and cancer mortality after different types of accelerated radiotherapy would be most valuable. Additionally, 
further research is required to examine for instance whether it is safe to provide chemotherapy concurrently with accelerated radiotherapy, whether the benefits of modified fractionation are preserved in case of concurrent chemoradiotherapy and whether other well-studied types of accelerated once-daily high-dose radiotherapy are cost-effective.

\section{Methodological considerations}

In the first case study, an integrated model was developed to overcome the lack of comparative effectiveness research. This integrated model consisted of individual patient characteristics, a dose-response model (e.g. NTCP model) and an economic model to assess the cost and consequences. In this case (Figure 7.1), patient-specific radiation dose to the swallowing structures and parotid glands was estimated (model 1) and linked to dose-response models which predict the probability of dysphagia and xerostomia (model 2). Subsequently, these patient-specific complication probabilities were combined with $\mathrm{HRQOL}$ and cost data to estimate the effectiveness and cost-effectiveness for individual patients (model 3).

This integrated model could also act as a decision support tool to facilitate individualized decision-making both for individualized treatment decisions in clinical practice (micro-level) and for individualized reimbursement decisions (macro-level). This methodology is based on surrogate outcomes and assumptions about reality and thus cannot be considered as a substitute for prospective clinical studies, rather a supplement. The methodology offers a solution if it is not desirable to wait or postpone decisions until clinical data become available. This can be the case for example in an early stage of a technology or if randomized trials are considered unethical or unfeasible. Moreover, as with decisionanalytic modelling in general, the integrated model might be employed to enrich, extrapolate and broaden the results from available evidence by combining various evidence sources and types of analytical models. ${ }^{2}$ Hence, even if comparative effectiveness research is available, the integrated modelling methodology might be used to address policy questions. ${ }^{3}$

As with most novel methodologies, the presented methodology is not yet validated. Moreover, the validity of the integrated modelling methodology heavily relies on the validity of the employed dose-response models. To critically appraise the validity of these dose-response models, the following dimensions of validity should be considered: face validity, internal validity, external validity and clinical validity. ${ }^{4}$ As part of the external validity, it was unclear in the first case study whether the NTCP models validated with photon studies can be used to predict toxicity after proton radiotherapy. Next to the dependence on the validity 


\section{INTEGRATED MODEL}

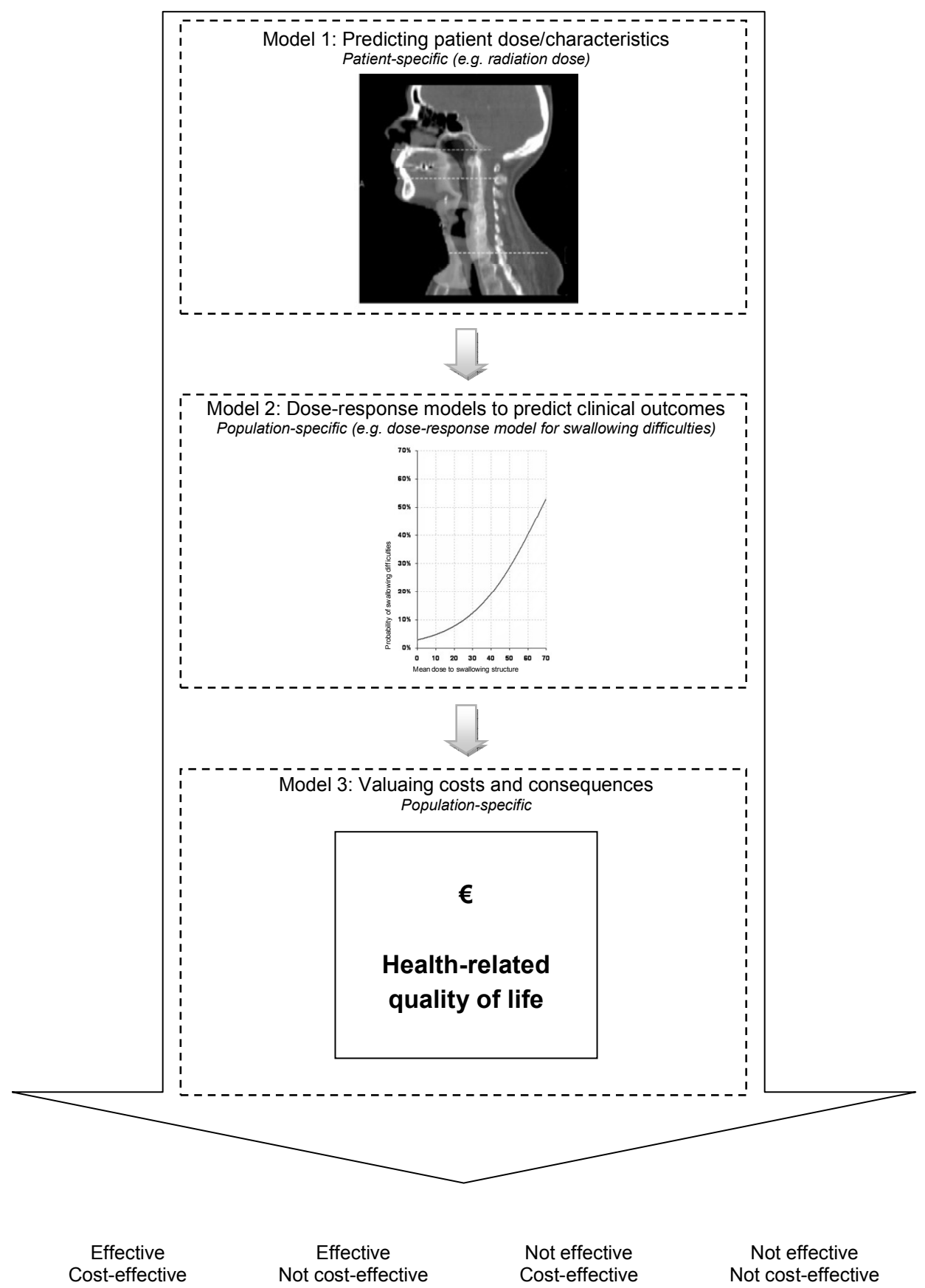

Figure 7.1: Integrated model to predict patient-specific effectiveness and cost-effectiveness 
of available NTCP models, two fundamental methodological challenges concerning the internal validity are still to be overcome before the integrated modelling methodology can be implemented on a broader scale. This involves examining 1) how valid and uncertain the individual predicted outcomes are for individuals; and 2) whether this methodology can be applied beyond radiotherapy. Firstly, the integrated model is partly based on population-specific data (e.g. Model 2) and does only reflect uncertainty surrounding specific model parameters (parameter uncertainty). It is questionable how these population-specific data should be incorporated to produce valid outcomes and appropriately reflect all existing uncertainty for individual patients. For example, additional uncertainty arises because population estimates are used to predict outcomes for specific individuals. One possible solution might be to incorporate unexplained between-subject variance in the integrated model to estimate the uncertainty of (sub)group predictions for individuals. Secondly, since dose-response models are not specific to radiotherapy, the wider applicability of this methodology, for instance in pharmacology, is an area for further research. In pharmacology for instance, population pharmacokinetic modelling ${ }^{5-8}$ can be used to optimize drug dosing for antibiotics. ${ }^{9}$ Additionally, dose-response models can be used to predict the probability of an event or disease ('response') based on biomarker values ('dose'). These prediction models can be used to facilitate personalized medicine. For instance, biomarkers related to thrombosis might be considered in the integrated model to select optimal thresholds when the risk of thrombosis becomes 'too high' and antithrombotic medication should be initiated. However, the feasibility and wider applicability of this methodology beyond radiotherapy should be confirmed in further research.

The second case study illustrated that if individual patient data are available, patient heterogeneity can be incorporated by using regression models to estimate input parameters dependent on patient characteristics. Individual patient data as available in the second case are preferred above surrogate (dosimetric) outcomes as in the first case study as these contain final outcomes and the highest grade of evidence. ${ }^{10}$ Additionally, individual patient data provide more opportunities to identify subgroups to be considered in the analysis, to explore patient heterogeneity based on identified subgroups, to examine for which subgroups additional research is valuable and to determine the optimal number of subgroups. ${ }^{11}$ Thus, to acknowledge patient heterogeneity and move towards personalized medicine, access to individual patient data is essential. Ideally, trial data should be made available to the public for this purpose. It can be questioned who should have the right to access the individual patient data. Data on what works in clinical practice can be considered a public good, independently of the entity (public or private) responsible for collecting the data. ${ }^{12}$ When considering the massive amount of public money and public trust which are placed in medical products that are adopted in clinical practice, it seems reasonable to request access to the full data. ${ }^{13}$ It may even be argued that 
society should have access to these data since the society indirectly pays for industry-funded trials through patents. To stimulate this openness of data, governments should establish laws that go beyond current legislation to demand access to the individual patient data behind any analyses used to license or reimburse a medical product. ${ }^{13}$ Additionally, scientific journals may facilitate this by using "open data" policy as for instance now applies to Nature journals: "authors are required to make materials, data and associated protocols promptly available to readers without undue qualifications". ${ }^{14}$ Ultimately, as put forward by others, this procedure should become the norm: "required by journals and accepted by the scientific community as mandatory". ${ }^{15}$ An "open data" approach is considered essential to maximize the benefits of research. ${ }^{15,16}$

In addition to "open data" policies, Performance-Based Risk Sharing Agreements (PBRSA) might provide opportunities to achieve data necessary for individualized decisions. Decision-makers often face considerable uncertainty about effectiveness and cost-effectiveness of novel medical products, and growing financial risks (due to the increasing costs of medical interventions). Partly in response to these concerns, the interest in PBRSA is substantial and rising. ${ }^{12}$ PBRSA are arrangements, often between the payer and producer of a medical product, mainly aimed at reducing the uncertainty through investing in the collection of evidence while a technology is temporarily adopted within clinical practice. These agreements concern a specified period of time and the (future) price and/or utilization of the product is dependent on the outcomes achieved. ${ }^{12,17}$ In this way, PBRSA may lead to value based pricing and facilitate patient access to (high cost) drugs or technologies. Part of the decision uncertainty aimed to resolve in PBRSA may originate from patient heterogeneity. These arrangements may thus provide an excellent opportunity for decisionmakers to request the collection of data necessary to examine the effectiveness and cost-effectiveness for subgroups of patients for instance identified in previous trials or based on biological plausibility. This may lead to ex ante reimbursement schemes, where the treatment is only reimbursed for a selection of individual patients for instance based on a biomarker or a diagnostic test. ${ }^{12}$ As an example: gefitinib for the treatment of non-small cell lung cancer is only recommended by National Institute for Health and Clinical Excellence for patients with a positive epidermal growth factor receptor mutation status and based on an agreed price. ${ }^{18}$ Alternatively, if it is not possible to identify subgroups before treatment initiation, PBRSA schemes focused on ex post reimbursement might prove valuable. This may involve schemes as "outcomes guarantees" meaning that the product will only be (fully) reimbursed for patients who respond to treatment or "conditional treatment continuation" meaning reimbursement for the continued use of a treatment based on intermediate outcomes. ${ }^{12}$ These arrangements seem promising as they potentially result in a number needed to treat of one. However, these ex post schemes require a 
measure for response or an intermediate outcome that is feasible, valid and can be measured relatively shortly after treatment initiation.

The data gathered through "open data" policy or PBRSA schemes may facilitate the acknowledgement of patient heterogeneity in decision-analytic models. This may involve complex methods ${ }^{19-23}$ and as reimbursement decision-makers are often not specialized in health economics, the cost-effectiveness results might become increasingly difficult to communicate. Therefore, to communicate the potential value of stratified or individualized decision-making and influence reimbursement policy, these complex methods should be translated to less complex outcomes. In the first case study, the EVIC was translated by using an additional comparator next to "proton radiotherapy for all patients" and "photon radiotherapy for all patients". This additional strategy was named "proton radiotherapy if efficient" and implies that patients for whom proton radiotherapy is expected to be cost-effective receive proton radiotherapy, while the remaining patients receive photon radiotherapy. The results of this strategy were presented as a 'normal' comparator using less complex outcomes such as the incremental cost-effectiveness ratio. Next to communicating outcomes to decisionmakers, the outcomes should also be properly communicated to clinical practice. For instance, the decision support tool as developed for the first case study can be converted to highly accessible smartphone apps or web-based applications. These applications can support physicians in their decisions without confronting them with the complexity of the decision-analytic model.

\section{Implications for policy}

Acknowledging patient heterogeneity may result in efficiency gains. ${ }^{23-26}$ Despite this potential efficiency gain, patient heterogeneity is currently frequently neglected in economic evaluations and subsequent policy decisions. ${ }^{27}$ Patient heterogeneity might be neglected in case it is considered unethical or controversial to exclude subgroups from treatment reimbursement based on certain characteristics. For instance, it may be considered unethical to discriminate patients and restrict access to treatment reimbursement based on differences in life expectancy (i.e. between younger and older patients). These considerations contain a normative trade-off between efficiency and equity. This also includes issues as whether subgroups should be excluded if a treatment is more effective but not cost-effective compared with current practice, or only when a treatment is not effective at all compared with current practice. These moral dilemmas should be handled in the technology appraisal by decision-makers and health authorities. Health authorities should provide guidance to researchers, for instance in the pharmacoeconomic guideline, when patient heterogeneity should (not) be acknowledged, how it should be acknowledged and what should 
be presented. Health authorities may consult the checklist presented in Chapter 2 to formulate guidance on acknowledging patient heterogeneity in their pharmacoeconomic guidelines.

To enable the acknowledgement of patient heterogeneity based on individual patient data, policy makers should stimulate the openness of data. This can for instance be part of the process to obtain a license or reimbursement for a medical product. Alternatively, in case individual patient data or comparative effectiveness research are not available, policy makers may consider other sources of evidence to acknowledge patient heterogeneity and inform their decisions (see for instance the first case study).

\section{Implications for clinical practice}

Personalized medicine might be enhanced if patient heterogeneity is acknowledged in economic evaluations and subsequent reimbursement decisions. Therefore, it is encouraged that acknowledging patient heterogeneity in economic evaluations becomes standard practice. In this way, economic evaluations might inform the development of clinical decision support tools. These tools can be used to examine which treatment is expected to be most (cost)effective for a specific patient. For instance, the first case study showed that proton therapy was only cost-effective in selected HNC patients for whom proton radiotherapy leads to a substantial reduction in the probability of treatment-related toxicity. The developed decision support tool may be used on the micro-level in clinical practice to select patients for treatment as well as on the macro-level to select patients for reimbursement. The second case study indicated that it is probably not valuable to individualize treatment decisions regarding radiotherapy fractionation scheme of NSCLC. Instead, it was encouraged to provide accelerated radiotherapy to all patients who do not receive concomitant chemo-radiotherapy.

\section{Concluding remarks and future perspectives}

To increase the efficiency of healthcare and bridge the gap between individualized decisions in clinical practice and population-based reimbursement decisions, it is encouraged that acknowledging patient heterogeneity becomes standard practice in future economic evaluations. Although this is preferably based on individual patient data, the unavailability of these data does not justify neglecting patient heterogeneity. It was demonstrated that acknowledging patient heterogeneity in economic evaluation is both feasible and informative, even if comparative effectiveness research is scarcely available. The interna- 
tional health economic community should develop consensus on how available methods such as stratified analysis ${ }^{23}$ regression techniques, ${ }^{19-21}$ and the expected value of individualized care $^{22}$ should be used to acknowledge patient heterogeneity in the economic assessment of technologies. Particularly international societies such as the International Society for Pharmacoeconomics and Outcomes Research (ISPOR) are encouraged to adopt a leading role in this process. On the other hand, national decision-makers are encouraged to provide specific recommendations in their pharmacoeconomic guideline when it is (ir)relevant (e.g. which characteristics can potentially be used for policy decision) to acknowledge patient heterogeneity in economic evaluations and what is required to inform their appraisal process.

One frequently mentioned barrier to acknowledging patient heterogeneity, a lack of appropriate data (e.g. if comparative clinical data or individual patient data are lacking), can be overcome by an integrated model using doseresponse models or over a longer period of time by risk sharing agreements and/or "open data" policy for scientific journals. Additionally, entities (public and private) responsible for collecting data are encouraged to make their data available to the public. This seems reasonable when considering the massive amount of public resources and trust that are placed in medical products adopted in clinical practice. Governments may facilitate this process by requesting openness of data for submissions during the licensing and reimbursing process of medical products. Additionally, future efforts should be aimed at further developing and validating the integrated modelling methodology, reaching consensus on how to acknowledge patient heterogeneity in the economic assessment and formulating specific guidance in pharmacoeconomic guidelines. This would increase population health gains and stimulate that health technology assessment practice and national reimbursement decisions are better aligned with the development towards personalized medicine in clinical practice. 


\section{References}

1. van der Laan HP, van de Water TA, van Herpt HE, et al: The potential of intensity-modulated proton radiotherapy to reduce swallowing dysfunction in the treatment of head and neck cancer: A planning comparative study. Acta Oncol 52:561-9, 2013

2. Brennan A, Akehurst R: Modelling in health economic evaluation. What is its place? What is its value? Pharmacoeconomics 17:445-59, 2000

3. O'Brien B: Economic evaluation of pharmaceuticals. Frankenstein's monster or vampire of trials? Med Care 34:DS99-108, 1996

4. Bentzen SM, Constine LS, Deasy JO, et al: Quantitative Analyses of Normal Tissue Effects in the Clinic (QUANTEC): an introduction to the scientific issues. Int $\mathrm{J}$ Radiat Oncol Biol Phys 76:S3-9, 2010

5. Colucci P, Grenier J, Yue CS, et al: Performance of different population pharmacokinetic algorithms. Ther Drug Monit 33:583-91, 2011

6. Jelliffe RW: Some comments and suggestions concerning population pharmacokinetic modeling, especially of digoxin, and its relation to clinical therapy. Ther Drug Monit 34:368-77, 2012

7. Kiang TK, Sherwin CM, Spigarelli MG, et al: Fundamentals of population pharmacokinetic modelling: modelling and software. Clin Pharmacokinet 51:515-25, 2012

8. Neely MN, van Guilder MG, Yamada WM, et al: Accurate detection of outliers and subpopulations with Pmetrics, a nonparametric and parametric pharmacometric modeling and simulation package for R. Ther Drug Monit 34:467-76, 2012

9. Croes S, Koop AH, van Gils SA, et al: Efficacy, nephrotoxicity and ototoxicity of aminoglycosides, mathematically modelled for modelling-supported therapeutic drug monitoring. Eur $\mathrm{J}$ Pharm Sci 45:90-100, 2012

10. Atkins $D$, Best $D$, Briss PA, et al: Grading quality of evidence and strength of recommendations. BMJ 328:1490, 2004

11. Saramago P: The Value of Further Research: The Added Value of Individual-Level Data. Value in Health 15:A492, 2012

12. International Society for Pharmacoeconomics and Outcomes Research (ISPOR). PerformanceBased Risk-Sharing Arrangements - Good Practices for Design, Implementation and Evaluation [Final draft]. 2012.

13. Godlee F, Clarke M: Why don't we have all the evidence on oseltamivir? BMJ 339:b5351, 2009

14. Nature Publishing Group: Availability of data and materials Accessed on January 15th, 2013. http://www.nature.com/authors/policies/availability.html

15. Boulton G: Open your minds and share your results. Nature 486:441, 2012

16. Garattini S, Bertele V: Europe's opportunity to open up drug regulation. BMJ 340:c1578, 2010

17. De Pouvourville G: Risk-sharing agreements for innovative drugs: a new solution to old problems? Eur J Health Econ 7:155-7, 2006

18. National Institute for Health and Clinical Excellence. Gefitinib for the first line treatment of locally advanced or metastatic non-small cell lung cancer. 2010 Technology Appraisal 192.

19. Hoch JS, Briggs AH, Willan AR: Something old, something new, something borrowed, something blue: a framework for the marriage of health econometrics and cost-effectiveness analysis. Health Econ 11:415-30, 2002

20. Nixon RM, Thompson SG: Methods for incorporating covariate adjustment, subgroup analysis and between-centre differences into cost-effectiveness evaluations. Health Econ 14:1217-29, 2005

21. Willan AR, Briggs $A H$, Hoch JS: Regression methods for covariate adjustment and subgroup analysis for non-censored cost-effectiveness data. Health Econ 13:461-75, 2004

22. Basu A, Meltzer D: Value of information on preference heterogeneity and individualized care. Med Decis Making 27:112-27, 2007

23. Coyle D, Buxton MJ, O'Brien BJ: Stratified cost-effectiveness analysis: a framework for establishing efficient limited use criteria. Health Econ 12:421-7, 2003 
24. Sculpher M: Subgroups and heterogeneity in cost-effectiveness analysis. Pharmacoeconomics 26:799-806, 2008

25. Zaric GS: The impact of ignoring population heterogeneity when Markov models are used in cost-effectiveness analysis. Med Decis Making 23:379-96, 2003

26. Stevens W, Normand C: Optimisation versus certainty: understanding the issue of heterogeneity in economic evaluation. Soc Sci Med 58:315-20, 2004

27. Jain R, Grabner M, Onukwugha E: Sensitivity analysis in cost-effectiveness studies: from guidelines to practice. Pharmacoeconomics 29:297-314, 2011 
SAMENVATTING 
S A M E N V A T T IN G 
Hoewel de mensheid als soort wordt gedefinieerd door een groot aantal gelijkenissen, bestaat er een aanzienlijke diversiteit tussen individuele patiënten. Alle patiënten zijn individuen en reageren vaak verschillend op identieke behandelingen ondanks een vergelijkbare diagnose. Deze natuurlijke variatie tussen patiënten kan worden gedefinieerd als variabiliteit. Helaas kunnen we deze variabiliteit vaak maar voor een klein deel voorspellen. Het deel van deze variabiliteit dat verklaard kan worden door bepaalde patiëntkenmerken wordt gedefinieerd als patiënt heterogeniteit. Deze patiëntkenmerken kunnen demografische gegevens, voorkeuren en klinische kenmerken omvatten. In de klinische praktijk wordt deze patiënt heterogeniteit steeds meer erkend door te anticiperen op voorspelbare verschillen in behandelrespons en hiermee te streven naar geïndividualiseerde behandelingen; 'de juiste behandeling voor de juiste persoon'. De belofte van geïndividualiseerd behandelen is dat het zal resulteren in een verbeterde effectiviteit en een vermindering van bijwerkingen en kosten. Evenals het erkennen van patiënt heterogeniteit in de klinische praktijk, kan het erkennen van patiënt heterogeniteit in economische evaluaties gunstig zijn. Dit kan potentieel resulteren in verbeteringen van de doelmatigheid en/of de effectiviteit van de gezondheidzorg. Patiënt heterogeniteit wordt echter vaak genegeerd in economische evaluaties en dus ook in het hierop gebaseerde beleid zoals besluiten omtrent het wel of niet vergoeden van bepaalde zorg. Dit proefschrift richt zich op de rol van patiënt heterogeniteit in economische evaluatie(s). De hypothese van dit proefschrift is dat het haalbaar en informatief is om patiënt heterogeniteit te erkennen in economische evaluaties.

In Hoofdstuk 2 wordt een systematische review gepresenteerd van aanbevelingen uit nationale farmaco-economische richtlijnen betreffende het erkennen van patiënt heterogeniteit in economische evaluaties. In totaal werden 26 farmaco-economische richtlijnen geïncludeerd waarvan er 20 adviseren om patiënt heterogeniteit te identificeren. Op basis van deze review kon worden geconcludeerd dat het belang van het erkennen van patiënt heterogeniteit in de economische evaluatie internationaal wordt bevestigd. Echter, het is minder duidelijk wanneer en hoe patiënt heterogeniteit precies moet worden erkend in economische evaluaties. Dit heeft onder andere betrekking op welke parameters of welke patiëntkenmerken relevant worden geacht om te erkennen in economische evaluaties, of gestratificeerde analyses vooraf moeten worden gespecificeerd en hoe subgroepen moeten worden gedefinieerd. Tot slot was het onduidelijk hoe de resultaten moeten worden gepresenteerd voor het informeren van geïndividualiseerde vergoedingsbesluiten. Gebaseerd op deze bevindingen, raden we aan dat toekomstige farmaco-economische richtlijnen specifieke aanbevelingen geven met betrekking tot het identificeren van patiënt heterogeniteit, methoden om patiënt heterogeniteit te erkennen en de presentatie van de resultaten. De gepresenteerde checklist kan autoriteiten ondersteunen om hun aanbevelingen te formuleren. Dit kan wereldwijd het erkennen van patiënt heterogeniteit in economische evaluaties faciliteren. 
De hierop volgende drie hoofdstukken hebben betrekking op de eerste casus waarin patiënt heterogeniteit wordt erkend. Het betreft een vergelijking van radiotherapie met protonen en radiotherapie met fotonen voor hoofd-halskanker patiënten. Het doel van deze casus was het erkennen van patiënt heterogeniteit in een economische evaluatie, in het geval vergelijkende studies met betrekking tot effectiviteit ontbreken. Hoofdstuk 3 betreft een synthese en vergelijking van het beschikbare bewijs omtrent de effectiviteit van radiotherapie met koolstofionen, protonen en fotonen voor hoofd-halskanker. Hiervoor werd een systematische review en meta-analyse uitgevoerd. In totaal werden 86 observationele studies ( 74 fotonen, 5 koolstof-ionen en 7 protonen) en acht vergelijkende insilico studies geïncludeerd. Aangezien de kwantiteit en de kwaliteit van de gegevens met betrekking tot radiotherapie met koolstof-ionen en protonen over het algemeen slecht was en gezien het risico op bias in vergelijkingen tussen (kleine) observationele studies, werden er geen definitieve conclusies geformuleerd. Ondanks deze tekortkomingen, kon er gesuggereerd worden dat een klinisch relevant voordeel van protonentherapie bij patiënten met hoofdhalskanker waarschijnlijk ligt in het verminderen van de bijwerkingen van de behandeling. Om in de toekomst definitieve conclusies te kunnen formuleren werd aanbevolen een internationaal register op te zetten voor het verzamelen van gegevens betreffende de effectiviteit van radiotherapie met koolstof-ionen en protonen.

Hoofdstuk 4 betreft een analyse van de impact van late behandeling gerelateerde xerostomie (droge mond) en dysfagie (slikklachten) op generieke gezondheidsgerelateerde kwaliteit van leven $(\mathrm{KvL})$ bij hoofd-halskanker patiënten behandeld met radiotherapie. Een multicenter cross-sectioneel onderzoek ( $N=$ 396) werd uitgevoerd. Het optreden van zowel xerostomie als dysfagie was geassocieerd met een substantieel verminderde $\mathrm{KvL}$; waarbij dysfagie de sterkste invloed had op KvL. Deze bevinding benadrukt dat het belangrijk is om naast de verwachte duur van de overleving, ook behandel-toxiciteit en het effect hiervan op KvL moet worden meegenomen wanneer behandelkeuzes worden gemaakt. De resultaten gepresenteerd in dit hoofdstuk kunnen worden gebruikt om deze afweging tussen de lengte en kwaliteit van de overleving te faciliteren. In deze overweging dient mee te worden genomen dat xerostomie vaker voorkomt dan dysfagie, terwijl dysfagie een grotere impact heeft op de KvL dan xerostomie. Daarom kunnen meer patiënten profiteren indien xerostomie kan worden vermeden, terwijl het vermijden van dysfagie misschien resulteert in een groter voordeel per patiënt.

De resultaten van de voorgaande twee hoofdstukken werden gebruikt in Hoofdstuk 5 om een modelmatige economische evaluatie uit te voeren om de effectiviteit en kosteneffectiviteit van protonentherapie te verkennen in vergelijking met radiotherapie met fotonen voor hoofd-halskanker patiënten. Protonentherapie is een veelbelovende radiotherapie modaliteit met een superieure dosisverdeling in vergelijking met radiotherapie met fotonen, maar is ook geas- 
socieerd met hogere kosten dan radiotherapie met fotonen. Deze kosten bestaan voornamelijk uit onomkeerbare investeringskosten. Zoals hierboven beschreven, was vergelijkend effectiviteitsonderzoek slechts beperkt beschikbaar voor radiotherapie met protonen bij hoofd-halskanker. Dit maakte het een uitdaging om de effectiviteit en kosteneffectiviteit te onderzoeken en het bemoeilijkte ook het erkennen van patiënt heterogeniteit in deze economische evaluatie. Om deze kloof van bewijsmateriaal te overbruggen werden dosisrespons modellen gecombineerd met vergelijkende planning studies in een geïntegreerd model. Dosis-respons modellen schatten de waarschijnlijkheid van het optreden van toxiciteit gebaseerd op de verwachte stralingsdosis in gezonde weefsels. Vergelijkende planning studies vergelijken voor verschillende radiotherapie technieken de verwachte stralingsdosis voor individuele patiënten. Daarom kunnen dosis-respons modellen en vergelijkende planning studies worden gebruikt in economische evaluaties om het te verwachten voordeel van innovatieve radiotherapie technieken te schatten voor individuele patiënten. Deze methode werd toegepast om patiënt heterogeniteit te erkennen in de gepresenteerde economische evaluatie bij gebrek aan vergelijkend effectiviteitsonderzoek. Er kon worden geconcludeerd dat protonentherapie gemiddeld niet doelmatig is. Echter, gekeken naar de individuele kosteneffectiviteit is protonentherapie mogelijk doelmatig voor individueel geselecteerde patiënten. Daarom werd het erkennen van patiënt heterogeniteit in deze casus als waardevol beschouwd voor het informeren van behandelbeslissingen op individueel niveau; dus voor het selecteren van patiënten voor wie protonentherapie doelmatig is en voor welke patiënten niet. Bovendien, gaf de "value of information" analyse aan dat verder onderzoek voor het verkrijgen van meer gegevens betreffende $\mathrm{KvL}$ na xerostomie en dosis-respons modellen om xerostomie en/of dysfagie te voorspellen het meest waardevol is. Verder zou het waardevol zijn om te verkennen welke bestralingstechniek het meest optimaal gebruik maakt van de dosimetrische voordelen van radiotherapie met protonen. De vergelijkende planning studie die gebruikt is in de huidige analyse was vooral gericht op het verminderen van de geschatte stralingsdosis in de slikstructuren. Het zou informatief kunnen zijn om de kosteneffectiviteit te schatten op basis van vergelijkende planning studies gericht op het verminderen van de geschatte stralingsdosis in de speekselklieren om het optreden van xerostomie te verminderen. Bovendien kunnen dosis-respons modellen ook gebruikt worden om strategieën te overwegen waarbij de verwachte totale dosis in de tumor wordt verhoogd om de kans op tumorcontrole te verbeteren.

De economische evaluatie van de tweede casus werd gepresenteerd in Hoofdstuk 6. Deze modelmatige economische evaluatie schat de kosteneffectiviteit van radiotherapie met alternatieve fractionering in vergelijking met conventioneel gefractioneerde radiotherapie voor patiënten met een niet-kleincellig longcarcinoom, die curatief behandeld worden zonder chirurgische resectie. In tegenstelling tot het vorige hoofdstuk, waar vergelijkend effectiviteitsonderzoek 
ontbrak, waren individuele patiëntengegevens van tien gerandomiseerde studies beschikbaar voor deze casus. In deze casus werd vastgesteld dat geaccelereerde radiotherapie gemiddeld de meest kosteneffectieve behandelingsstrategie was voor alle patiënten. De "expected value of individualized care" gaf aan dat, in deze casus, het nemen van beslissingen op individueel niveau waarschijnlijk niet waardevol is. Dus geaccelereerde radiotherapie was de meest doelmatige strategie voor alle patiënten en het leek niet waardevol om behandelbesluiten te individualiseren met betrekking tot het type fractioneringsschema. Echter, zijn deze resultaten waarschijnlijk het meest van toepassing op patiënten die met radiotherapie behandeld worden zonder gelijktijdige chemotherapie. Dit omdat deze gelijktijdige behandel combinatie in slechts twee van de tien studies werd gegeven en het onduidelijk is of de voordelen van radiotherapie met alternatieve fractionering worden behouden indien radiotherapie gelijktijdig wordt gegeven met chemotherapie. Daarnaast zijn de verschillen in effectiviteit tussen de behandelingen klein en was het onzeker welk type geaccelereerde radiotherapie het meest doelmatig is. Niettemin is geaccelereerde radiotherapie vrijwel zeker doelmatiger dan de huidige praktijk (conventioneel gefractioneerde radiotherapie). Wachten op meer bewijs voordat geaccelereerde radiotherapie wordt geïmplementeerd zou daarom leiden tot een minder hoge gezondheidswinst. Bovendien, wanneer toekomstig onderzoek zou aantonen dat geaccelereerde radiotherapie niet het meest kosteneffectieve type radiotherapie zou zijn, zijn de onomkeerbare investeringskosten naar verwachting laag. Daarom wordt aanbevolen om geaccelereerde radiotherapie te implementeren als standaard behandeling voor de curatieve behandeling van patiënten met een niet-kleincellig longcarcinoom die behandeld worden zonder chirurgische resectie en gelijktijdige chemotherapie. Verder gaf de "value of information" analyse aan dat verder onderzoek naar kosten van de initiële radiotherapie behandeling en kanker gerelateerde sterfte het meeste waardevol is. Daarnaast is verder onderzoek vereist om bijvoorbeeld te onderzoeken of de voordelen van alternatieve fractionering worden behouden bij gelijktijdige chemotherapie en om de doelmatigheid van andere geaccelereerde radiotherapie types te bestuderen.

Hoofdstuk 7 betreft de algemene discussie die eindigt met een slotbeschouwing en toekomstperspectieven. Hierin wordt gepleit voor het erkennen van patiënt heterogeniteit als standaard praktijk in toekomstige economische evaluaties. Dit zal de doelmatigheid van de gezondheidszorg verbeteren en de kloof verkleinen tussen geïndividualiseerde beslissingen in de klinische praktijk en populatie-gebaseerde vergoedingsbeslissingen. Hoewel dit bij voorkeur wordt gedaan op basis van individuele patiëntgegevens, is het niet gerechtvaardigd patiënt heterogeniteit te negeren omdat deze gegevens niet beschikbaar zijn. In dit proefschrift wordt aangetoond dat het erkennen van patiënt heterogeniteit in de economische evaluatie haalbaar en informatief is, zelfs als vergelijkend effectiviteitsonderzoek nauwelijks beschikbaar is. De internationale gezond- 
heidseconomische gemeenschap zou tot een consensus moeten komen over hoe beschikbare methoden zoals gestratificeerde analyse, regressie technieken en de "expected value of individualized care" moeten worden gebruikt om patiënt heterogeniteit te erkennen in economische evaluaties. Vooral internationale gemeenschappen, zoals de International Society for Pharmacoeconomics en Outcomes Research (ISPOR), worden aangemoedigd om hierin een leidende rol te nemen. Aan de andere kant worden nationale beleidsmakers aangespoord om specifieke aanbevelingen te geven in hun farmaco-economische richtlijnen wanneer het (ir)relevant is om patiënt heterogeniteit te erkennen in economische evaluaties (bijvoorbeeld welke kenmerken eventueel gebruikt kunnen worden voor beleidsbeslissing) en wat nodig is om hun beslissingen te ondersteunen.

Een vaak genoemde belemmering voor het erkennen van patiënt heterogeniteit is een gebrek aan geschikte gegevens (bijvoorbeeld als vergelijkende klinische gegevens of gegevens van individuele patiënten ontbreken) en kan verholpen worden door een geïntegreerd model met behulp van dosis-respons modellen of over een langere periode door "risk sharing" overeenkomsten en/of "open data" beleid voor wetenschappelijke tijdschriften. Daarnaast worden organisaties (publieke en private) die verantwoordelijk zijn voor het verzamelen van gegevens uitgenodigd om hun gegevens beschikbaar te maken voor de maatschappij. Dit lijkt redelijk gezien de enorme hoeveelheid publieke middelen die geïnvesteerd worden in medische producten en het grote vertrouwen dat in deze producten gesteld wordt. Overheden kunnen bijdragen aan dit proces door te vragen naar openheid van gegevens tijdens het proces voor het verkrijgen van de licentie en de vergoeding van medische producten. Hiernaast is verder onderzoek noodzakelijk voor het verder ontwikkelen en valideren van de geïntegreerde modelleringsmethodologie, het bereiken van consensus over de wijze waarop patiënt heterogeniteit zou moeten worden erkend in economische evaluaties en het formuleren van specifieke aanbevelingen in farmacoeconomische richtlijnen. Dit zou resulteren in een toegenomen gezondheidswinst op populatieniveau en het zou bevorderen dat economische evaluaties en nationale vergoedingsbesluiten beter worden afgestemd op de ontwikkeling in de klinische praktijk richting gepersonaliseerde geneeskunde. 

PUBLICATIONS 
PUBLICATIONS 


\section{Peer reviewed scientific publications}

Ramaekers BL, Joore MA, Lueza B, Bonastre J, Mauguen A, Pignon J-P, Le Pechoux C, De Ruysscher D, Grutters JP. Cost-effectiveness of modified fractionation radiotherapy versus conventional radiotherapy for unresected nonsmall cell lung cancer patients. J Thorac Oncol. Accepted for publication.

Ramaekers BL, Joore MA, Grutters JP. How should we deal with patient heterogeneity in economic evaluation according to pharmacoeconomic guidelines? Value in Health. In press.

Ramaekers BL, Grutters JP, Pijls-Johannesma M, Lambin P, Joore MA, Langendijk JA. Protons in head and neck cancer: bridging the gap of evidence. Int J Radiat Oncol Biol Phys. 2013;85(5):1282-8

Grutters JP, Sculpher M, Briggs AH, Severens JL, Candel MJ, Stahl JE, De Ruysscher A, Boer A, Ramaekers BL, Joore MA. Acknowledging patient heterogeneity in economic evaluation: a systematic literature review. PharmacoEconomics. 2013;31(2):111-23

Ramaekers BL, Joore MA, Grutters JP, Van den Ende P, De Jong J, Houben $R$, Lambin P, Christianen M, Beetz I, Pijls-Johannesma M, Langendijk JA. The impact of late treatment-toxicity on generic health-related quality of life in head and neck cancer patients after radiotherapy. Oral Oncol. 2011;47(8):768-74

Ramaekers BL, Pijls-Johannesma M, Joore MA, Van den Ende P, Langendijk JA, Lambin P, Kessels AG, Grutters JP. Systematic review and meta-analysis of radiotherapy in various head and neck cancers: comparing photons, carbonions and protons. Cancer Treat Rev. 2011;37:185-201

Lambin P, Ramaekers BL, van Mastrigt GA, Van den Ende P, de Jong J, De Ruysscher DK, Pijls-Johannesma M. Erythropoietin as an adjuvant treatment with (chemo) radiation therapy for head and neck cancer. Cochrane Database Syst Rev. 2009;8(3)

Ramaekers BL, Janssen-Boyne JJ, Gorgels AP, Vrijhoef HJ. Adherence among telemonitored patients with heart failure to pharmacological and nonpharmacological recommendations. Telemed J E Health. 2009;15(6):517-24

\section{Presentations at (inter)national conferences}

Ramaekers BL, Joore MA, Grutters JP. Acknowledging heterogeneity in Health Technology Assessment to improve efficient use of pharmaceuticals. (Invited oral presentation) HTA-methodologiedag, April 16, 2013 (Utrecht, The Netherlands) 
Ramaekers BL, Joore MA, Grutters JP. How should we deal with patient heterogeneity in economic evaluation according to pharmacoeconomic guidelines? (Oral presentation) European Conference on Health Economics, July 18-21, 2012 (Zürich, Switzerland)

Ramaekers BL, Joore MA, Grutters JP. Acknowledging patient heterogeneity in economic evaluation: a systematic review of guidance from pharmacoeconomic guidelines. (Oral presentation) Health Technology Assessment International, June 25-27, 2012 (Bilbao, Spain)

Ramaekers BL, Joore MA, Grutters JP. Patient heterogeneity in economic evaluations: how should we handle it according to pharmacoeconomic guidelines? (Discussion session) The Low Lands Health Economists' Study Group (LoLaHESG), May 24-25, 2012 (Almen, The Netherlands)

Ramaekers BL, Grutters JP, Pijls-Johannesma M, Lambin P, Langendijk JA, Joore MA. Cost-effectiveness analysis of innovative radiotherapy techniques in the absence of comparative effectiveness research: the case of proton radiotherapy in head and neck cancer. (Oral presentation) International Health Economics Association, July 10-13, 2011 (Toronto, Canada)

Ramaekers BL, Joore MA, Grutters JP, Van den Ende P, De Jong J, Christianen M, Beetz I, Lambin P, Pijls-Johannesma M, Langendijk JA. Impact of xerostomia and dysphagia on health-related quality of life in head and neck cancer patients. (Poster presentation) European Society for Therapeutic Radiology \& Oncology, September 12-16, 2010 (Barcelona, Spain)

Ramaekers BL, Pijls-Johannesma M, Joore MA, Van den Ende P, Langendijk JA, Lambin P, Kessels AG, Grutters JP. Radiotherapy with photons, carbonions and protons in various head and neck cancers: a review and meta-analysis of observational studies. (Poster presentation) European Society for Therapeutic Radiology \& Oncology, September 12-16, 2010 (Barcelona, Spain)

\section{Published abstracts}

De Ruysscher D, Ramaekers BL, Joore MA, Lueza B, Bonastre J, Mauguen A, Pignon J, Le Pechoux C, Grutters JP, on behalf of The Mar-LC Collaborative Group. Modified fractionation radiotherapy versus conventional radiotherapy for unresected non-small cell lung cancer patients: a cost-effectiveness analysis. Ann Oncol. 2012;23 Supplement 9, ix389-ix399

Ramaekers BL, Joore MA, Grutters JP. Acknowledging patient heterogeneity in economic evaluation: A systematic review of guidance from pharmacoeconomic guidelines. Gac Sanit. 2012; 26, 31 
Houben R, Pijls-Johannesma M, Ramaekers BL, Van den Ende P, De Jong J, De Ruysscher DK, Lambin. P. Erythropoietin as an adjuvant treatment with (chemo) Radiation therapy for head and neck cancer: Up-dated systematic review with additional data and new methodology. Radiother Oncol. 2010;96 Supplement 1, S78

Ramaekers BL, Joore MA, Grutters JP, Van den Ende P, De Jong J, Christianen M, Beetz I, Lambin P, Pijls-Johannesma M, Langendijk JA. Impact of xerostomia and dysphagia on health-related quality of life in head and neck cancer patients. Radiother Oncol. 2010; 96 Supplement 1, S318

Ramaekers BL, Pijls-Johannesma M, Joore MA, Van den Ende P, Langendijk JA, Lambin P, Kessels AG, Grutters JP. Radiotherapy with photons, carbonions and protons in various head and neck cancers: a review and meta-analysis of observational studies. Radiother Oncol. 2010; 96 Supplement 1, S540-S541

Boyne JJ, Ramaekers BL, De Wit R, Gorgels AP, Vrijhoef HJ. Adherence to medication and non-pharmacological recommendations in telemonitored heart failure patients. Eur J Heart Fail. 2009; 8 Supplement 2, ii564

\section{Grants / awards}

Travel grant from Limburg University Fund / SWOL

Particle Therapy Co-Operative Group (PTCOG) travel fellowship 



\section{DANKWOORD}


DANKWOORD 
Het is zover, het boekje is af! Maar dat heb ik natuurlijk niet alleen gedaan. Er waren veel mensen en organisaties betrokken bij de totstandkoming van dit proefschrift. Graag wil ik iedereen, en een aantal mensen in het bijzonder, ontzettend bedanken voor hun bijdrage aan mijn avontuur door de wondere wereld die onderzoek heet.

Allereerst wil ik de patiënten bedanken die aan de kwaliteit van leven studie hebben deelgenomen (hoofdstuk 4 van dit proefschrift). Hartelijk dank voor het invullen van de vragenlijsten.

Mijn promotor: Philippe Lambin. Beste Philippe, ik ben je zeer dankbaar voor de geboden kansen en je vertrouwen in het projectteam. Verder is mijn promotieteam niet compleet zonder mijn copromotores: Janneke Grutters, Manuela Joore en Madelon Pijls-Johannesma. Jullie hebben mij geïntroduceerd en begeleid in dit wetenschappelijke avontuur. Dank voor alle kansen, vrijheid en vertrouwen die jullie mij hebben gegeven, maar bovenal voor het enthousiasme voor onderzoek dat jullie op mij hebben overgebracht. Beste Janneke, aan jouw kritische blik ontgaat niets, naast jouw deskundigheid en zorgvuldigheid is dit een essentiële kwaliteit als onderzoeker. Hoewel ik af en toe wel eens baalde na een van onze meetings omdat ik een stap terug moest, was het erg leerzaam en resulteerde het vaak in een sterk verbeterd eindresultaat. Nu ik terugkijk op het avontuur dat ze promoveren noemen, denk ik dat ik zelfs een deel van deze kritische blik heb overgenomen. Beste Manuela, ik heb veel bewondering voor jouw capaciteit om snel te schakelen en conceptuele manier om naar zaken te kijken. Hier kan ik nog veel van leren. Op die momenten dat ik graag te snel in de details duik, wees jij mij vaker op het grotere plaatje. Ik ben je ook zeer dankbaar voor de kans die jij mij hebt geboden om de laatste puntjes op de ' $i$ ' te zetten voor mijn proefschrift en me verder te ontwikkelen als onderzoeker na mijn promotie. Beste Madelon, jouw kennis, betrokkenheid en motiverende houding zijn erg waardevol geweest. Je hebt me altijd de ruimte en vrijheid gegeven om mijn eigen weg te zoeken. Alhoewel ik jullie alle drie erg dankbaar ben voor bovenstaande, is mij een vreemd verband opgevallen dat ik toch even met jullie wil delen. Nadat ik bij MAASTRO CLINIC startte aan mijn onderzoek, waar Madelon mijn $1^{\mathrm{e}}$ aanspreekpunt was, verwisselde Madelon na een tijdje van baan (van MAASTRO CLINIC naar CZ). Vervolgens zette ik mijn onderzoek voort bij de Universiteit Maastricht, waar Janneke mijn $1^{\mathrm{e}}$ aanspreekpunt was, en ook zij verwisselde na een tijdje van baan (van UM naar UMC St Radboud). Ik hoop dat we hier kunnen spreken van een toevalsbevinding en dat dit niet duidt op een statistisch significant verband. :)

De leden van de beoordelingscommissie: Prof. dr. Bernd Kremer (voorzitter), Dr. Thea van Asselt, Dr. Frank Hoebers en Prof. dr. Yolande Lievens wil ik graag bedanken voor het kritisch lezen en beoordelen van mijn proefschrift. 
Gedurende het promotietraject zijn er veel personen geweest waarmee ik prettig heb samengewerkt of gewoon regelmatig een leuk praatje mee kon maken. De eerste 2 jaar van mijn onderzoek heb ik bij MAASTRO CLINIC gewerkt. Ik wil graag de collega's van het DCM bedanken: Ann, Annette, Cary, Chantal, Merel, Ria, Ruud, Sylvia en later ook Iverna. Ook wil ik Bianca en Claudia bedanken voor de gezelligheid in de kantoortuin. Verder ben ik Piet van den Ende, Jos de Jong en Dirk De Ruysscher dankbaar voor hun klinische input en bijdrage als medeauteurs. Tevens wil ik ook de overige medewerkers (o.a. laboranten, onderzoekers, promovendi, artsen (in opleiding), fysici) van MAASTRO CLINIC en het oncologisch centrum waar ik vaker een praatje mee maakte of die hebben meegewerkt aan de kwaliteit van leven studie graag bedanken.

Fons, bedankt voor de ondersteuning in het 'foetelen' met data (andere mensen zouden het meta-analyse noemen), dit heeft mede mijn enthousiasme voor de kwantitatieve kant van onderzoek aangewakkerd.

Hans Langendijk, Ivo Beetz en Miranda Christianen wil ik graag bedanken voor de Maastricht-Groningen samenwerking.

Het tweede deel van mijn promotietraject was ik bij de Universiteit Maastricht werkzaam. Hier waren Anil en Mitchel mijn 'roommates'. Vooral met laatstgenoemde heeft dit tot eindeloze tafelvoetbal sessies en VVV - Ajax discussies geleid. Hierbij werd Mitchel meermaals (noodzakelijkerwijs natuurlijk) bijgestaan door mede Ajacied Arno. Ik ben nog steeds van mening dat je niet naar de absolute plaats op de ranglijst maar naar de relatieve prestaties van een voetbalclub moet kijken en dat deze ook nog eens afgezet dienen te worden tegen de begroting. Doordat afgelopen seizoen zelfs de relatieve prestaties van VVV onder de maat zijn geweest, Voetballen we Voorlopig op Vrijdag in Venlo, echter zie ik geen Reden om ervan Uit te gaan dat dit Langer dan Een Seizoen duurt. Verder, Anil nu ik je weer tegenkom bij KEMTA, kunnen we definitief een 'Dutch Day' invoeren. Hiernaast waren ook de bezoeken aan het 'meidenhok' van DUB30 bevolkt door: Reina, Janneke, Arianne en Cindy gezellig. Natuurlijk wil ik ook alle andere oud-collega's, het zijn er te veel om op te noemen, bedanken voor de prettige informele en/of formele samenwerking.

During the $3^{\text {rd }}$ year of my PhD, I visited Institut Gustave-Roussy in Paris and I would like to thank Béranger Lueza, Julia Bonastre, Jean-Pierre Pignon and Cecile Le Pechoux for the opportunities and fruitful collaboration which I hope to continue for many years. 
Verder wil ik ook mijn huidige collega's bij KEMTA bedanken voor de koffie momenten, inhoudelijke discussies en inspiratie de ik nodig had om mijn proefschrift af te ronden.

Het zijn eigenlijk te veel collega's en medeauteurs om op te noemen, maar ik wil ook diegene die ik onvermijdelijk vergeten ben bedanken voor de enerverende inhoudelijke gesprekken maar ook voor alle koffies, lunches, uitstapjes en overige activiteiten.

Een van de leuke dingen aan een avontuur is dat je het kunt delen; de (spannende) verhalen kunt vertellen aan familie en vrienden. Daarom wil ik ook mijn ouders, broertje en zusje bedanken voor hun luisterend oor, maar ook hun steun en vertrouwen in mij. Daarnaast wil ik alle familie en vrienden bedanken die mij hebben geholpen of gewoon gezellig voor 'afleiding' hebben gezorgd. Dat we nog veel feestjes, etentjes, whisky events of avonden in de kantine mogen beleven.

Tenslotte lieve Jennifer, bedankt voor je steun, begrip en liefde zodat ik dit avontuur, waarin voor jou ook een grote rol was weggelegd (kijk maar o.a. naar de kaft), heb kunnen beleven. 

CURRICULUM VITAE 
CURRICULUM VITAE 
Bram Ramaekers was born on January $28^{\text {th }}, 1986$, in Venlo, the Netherlands. In 2003, he graduated from the Blariacum College in Blerick. Subsequently, he started studying Medical Imaging and Radiation Therapy (MBRT) at the Fontys Hogescholen in Eindhoven. In the final year of this study (2006-2007), he worked on a regular basis as a radiation technologist at MAASTRO CLINIC, finished the pre-master methodology and statistics program at Maastricht University and successfully finished studying MBRT (Bachelor of Health). Afterwards he studied Public Health (specialization: Health Services Innovation) at Maastricht University. After obtaining his Master degree in 2008 (Master of Science), he started as a PhD student at MAASTRO CLINIC. In 2010 he continued his $\mathrm{PhD}$ research at Maastricht University at the department Health Organisation, Policy and Economics (currently department of Health Services Research). Since November 2012 Bram works as a researcher at Maastricht University Medical Center (department of Clinical Epidemiology and Medical Technology Assessment) where he is involved in multiple research projects related to Health Technology Assessment. This includes acting as a member of the Evidence Review Group for The National Institute for Health and Clinical Excellence (NICE). In addition, he is also involved in teaching and supervising master students. 


\section{When you're thankful for what you have, you are always rewarded with more.}

www.livelifehappy.com 